\title{
A Taxonomy of Inverse Priming Based on Stimulus Characteristics
}

\author{
Dissertation \\ zur Erlangung des mathematisch-naturwissenschaftlichen Doktorgrades \\ „Doctor rerum naturalium“ \\ der Georg-August-Universität Göttingen
}

vorgelegt von

Daniel Krüger

aus Steinheim, Westfalen 
Referent: Prof. Dr. Uwe Mattler

Koreferentin: Prof. Dr. Uta Lass

Tag der mündlichen Prüfung: 15.11.2011 


\section{Acknowlegdgements}

Many people have helped me working out the results reported in this thesis. First of all, I want to express a deep sense of gratitude to my guide and supervisor Prof. Uwe Mattler, who designed the presented studies with me and who gave me valuable input during all stages of my work. I'm greatly indepted to him for taking his time to discuss the results and to share part of his huge knowledge with me.

I also want to thank Prof. Uta Lass not only for reviewing this thesis but also for her support and the optimistic view of my work during the last years which encouraged me a lot.

I'm also very thankful for the support, cooperation and input from all the great people I worked with in the Abteilung für Experimentelle Psychologie at the Georg-Elias-Müller Institute for Psychology. In particular, I want to sincerely thank Susan Klapötke, Simon Palmer and Thorsten Albrecht for all their support, feedback and simply for the great time.

A special thanks also goes to the students who participated in the research projects performed in the context of this thesis: Nadja Arnold, Martina Wernicke and Nicolas Becker. Your reliable help was essential for the results.

I further thank the members of the research group 'MR research in Neurology and Psychiatry', especially Peter Dechent, Carsten Schmidt Samoa, Britta Perl and Ilona Pfahlert, for their assistance with running the fMRI experiment. Without their help this would not have been possible.

Finally, I would like to express my gratitude to Mercedes Cerezuela whom I'm much indepted for her invaluable support during all the years. 


\section{Summary}

One intriguing question in cognitive neuroscience is whether subliminal stimuli can affect overt behavior. A straightforward experimental approach to demonstrate effects of subliminal stimuli is the response priming paradigm. In response priming, a masked prime stimulus facililitates processing of targets which require the same response (congruent condition) as opposed to targets requiring an alternative response (incongruent condition). When prime visibility is reduced by an additional masking stimulus following the prime but preceding the target, priming effects are reversed. Then, performance costs are observed in the congruent condition and benefits in the incongruent condition. Various explanations for this couterintutive phenomenon have been proposed including perceptual, central and motor accounts. Empirical findings regarding the locus of inverse priming are divergent.

Five research projects have been performed in order to clarify the mechanism underlying the inverse priming effect. Psychophysical and physiological evidence support the view that inverse priming is generated in different ways according to the characteristics of the stimuli involved. When primes and targets are compatible to the responses assigned to them, like double arrows which require corresponding left-hand and right-hand responses, inverse priming seems to originate from the motor system. Probably, subliminal primes automatically activate the corresponding response which becomes inhibited due to the presentation of the masking stimulus finally leading to a bias for the incongruent response. In contrast, inverse priming with response non-compatible stimuli, like squares and diamonds, is supposed to be entirely generated at perceptual levels of processing. Furthermore, inverse priming with such stimuli seems to be limited to the use of masks which comprise task-relevant features. With such relevant masks, inverse priming presumably results from a perceptual interaction of primes and masks rendering prime-incongruent elements in the mask more salient which in turn facilitates perceptual processing of incongruent targets. With irrelevant masks, small but reliable effects have only been found in a non-motor priming task indicating that a further distinct mechanism might produce inverse priming.

Thus, inverse priming illustrates that a single observable phenomenon can be produced by various different mechanisms according to the stimuli employed. On a more abstract level, results suggest that the influence of subliminal stimuli goes beyond the simple activation of motor responses. Complex control processes seem to be triggered by subliminal stimuli which remain unconsicous. 


\section{Contents}

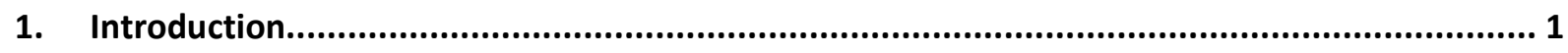

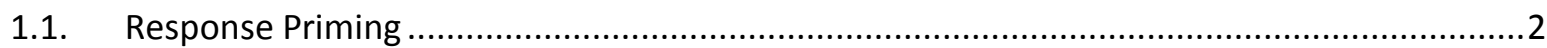

1.2. Inverse Priming .............................................................................................

1.3. Motor accounts of inverse priming ......................................................................

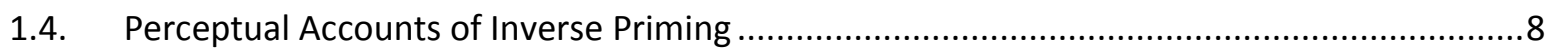

1.5. Central Accounts of Inverse Priming...................................................................

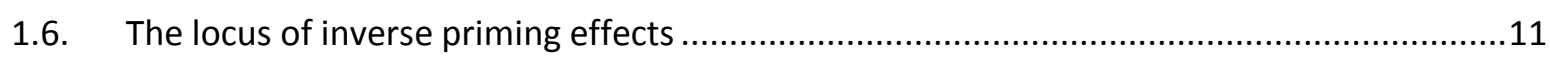

1.7. A taxonomy of inverse priming based on stimulus structure ...................................14

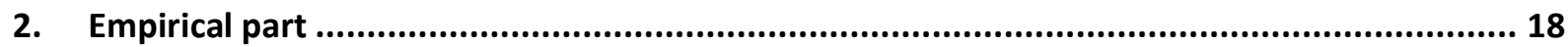

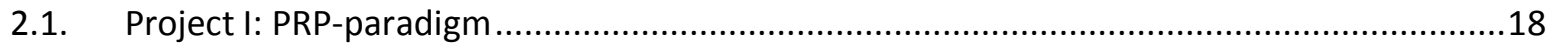

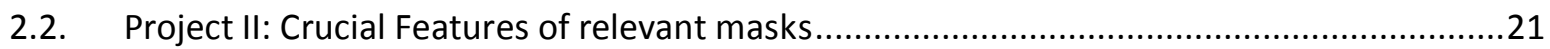

2.3. Project III: Stimulus-response compatibility and inverse priming.................................24

2.4. Project IV: Inverse non-motor priming ............................................................28

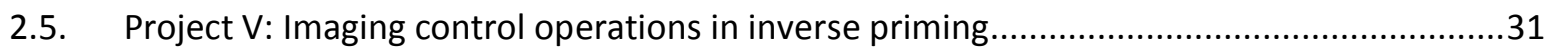

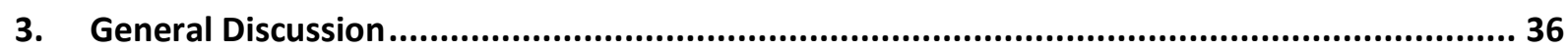

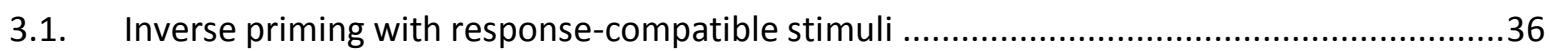

3.2. Are arrows special or does inverse priming result with all response-compatible stimuli? ...39

3.3. Inverse priming with response non-compatible stimuli ......................................40

3.4. Summary: At least two different mechanisms generating inverse priming ......................41

3.5. The role of prime visibility in inverse priming ........................................................45

3.6. A common mechanism of inverse priming with relevant and irrelevant masks .................48

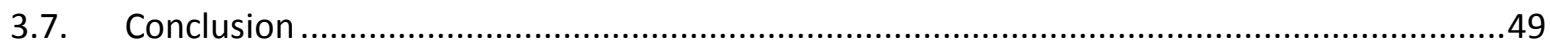

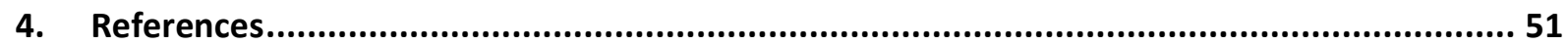

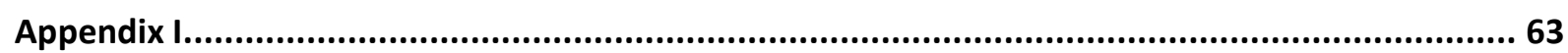

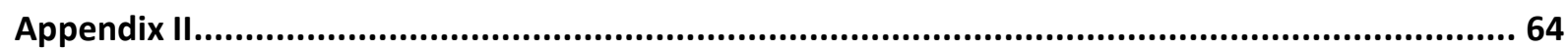

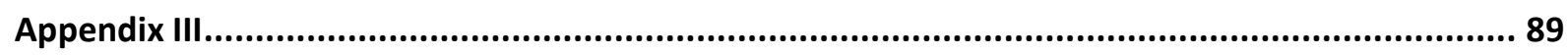

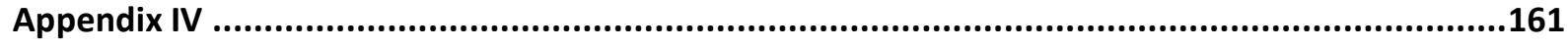

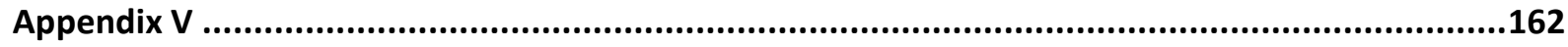

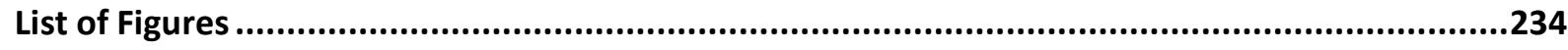

List of Abbreviations..............................................................................................235

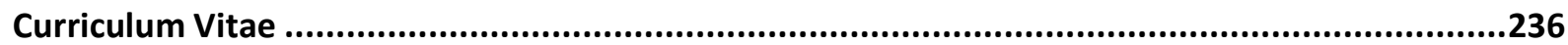




\section{Introduction}

Human behavior is the result of the complex interplay of various factors. One common distinction differentiates between internally and externally guided actions. Internally guided actions aim to produce a desired outcome and are thus subject to intentions like for example taking the newspaper to read it. In contrast, externally guided actions are prompted by environmental stimuli (Prinz, 1997), for example stopping the car in front of a red traffic light. Thus, externally guided actions help the subject to adapt his behavior to the current environmental demands. One of the most intriguing issues in cognitive neuroscience is the question to what extent such externally guided behavior can be controlled or at least influenced by subliminally presented visual stimuli. First evidence in favor of such a subliminal influence was derived from observations of patients suffering from a class of neurological disorders impairing their visual perception but leaving intact their capacity to respond to environmental stimuli in an adaptive manner. Thus, the translation of external stimuli into actual behavior, often termed the visuomotor processing of such stimuli, is spared (for a review see Goodale \& Milner, 1995, 2006). A classic example is patient DF suffering from visual form agnosia (Milner, et al., 1991; Goodale, Milner, Jakobsen, \& Carey, 1991). A carbon monoxide poisoning led to ventro-lateral lesions within the occipital cortex including cortical areas 18 and 19, but largely sparing area 17 . In a number of tasks her visuomotor processing of visual stimuli was found to be unaffected whereas visual recognition was severely impaired. For instance, she proved to be unable to report the orientation of a tilted slot (direct measure of conscious access, Reingold \& Merikle, 1988, 1993) but she could insert a card into that slot fast and accurately (indirect measure of the behavioral impact). In the same way, she was incapable of indicating the width of an object but she could easily adapt the hand aperture to the size of such an object grasping it just like normal subjects. Thus, demonstration of the subliminal stimulus processing involves a direct measure of conscious access to such a stimulus acquired in a so-called direct task, like demonstrating the width of the object, and a second measure derived from the so-called indirect task indicating that the subliminal stimulus actually affects behavior. Visual neglect (Berti, \& Rizzolatti, 1992) or blindsight (Weiskrantz, Warrington, Sanders, \& Marshall, 1974) constitute further examples for a dissociation of performance on the direct and indirect task. These patients show intact visuomotor processing of visual input indicated by some indirect behavioral effect but the very same visual information necessary for carrying out the required behavioral output is not directly consciously available to them. The fact that visuomotor performance is not exclusively driven by information present in conscious visual experience led Milner and 
Goodale (1995) to postulate the existence of two independent visual systems: conscious visual perception and visuomotor control which thereby implies that subliminal stimuli affect behavior through the latter system.

The cited neuropsychological evidence, however, suffers from a common methodological issue limiting their informative value for the investigation of subliminal stimulus processing. All the above-mentioned disorders constitute examples for a so-called simple dissociation between conscious access to a stimulus and visuomotor processing of the same stimulus (Schmidt, \& Vorberg, 2006). A simple dissociation is characterized by (1) some indirect behavioral effect of a stimulus while (2) the direct measure of recognition of that stimulus is at chance level. In such cases, however, conscious stimulus perception cannot be completely ruled out because the direct measure might not be exhaustive for awareness (Reingold, \& Merikle, 1993). In a recent example, healthy subjects proved to be unable to directly identify the shape of an object visually masked by a second following object. In contrast, on a different (direct) task they showed above chance-level performance since they could report a simple visual interaction of the shape of that object with the following second object in a movement discrimination task (Ansorge, Becker, \& Breitmeyer, 2009). These results indicate that different ways to obtain a measure for conscious availability might yield different results and that one measure might be more sensitive for residual stimulus information than another one. Therefore, an indirect behavioral effect of a subliminal stimulus could always be due to residual visual awareness. A simple dissociation is consequently not sufficient to prove the existence of subliminal stimulus processing. To overcome this problem, experimental manipulations which have opposing effects on conscious availability of stimulus information and visuomotor processing have been developed (Mattler, 2003; Vorberg, Mattler, Heinecke, Schmidt, \& Schwarzbach, 2003). Such an experimental manipulation creates a double dissociation implying that visuomotor processing and conscious perception reflect independent underlying processes (Schmidt, \& Vorberg, 2006). The response priming paradigm allows the experimental creation of such a double dissociation. It is further described in the following section.

\subsection{Response Priming}

Responding to a visual target stimulus is faster and less error-prone when a prime stimulus that is assigned to the same response has been encountered briefly before (congruent condition), as compared to a prime stimulus assigned to an alternative response (incongruent 
condition). This phenomenon is called (positive) response priming. Astonishingly, this indirect effect of the prime on behavioral performance is still found when the prime stimulus becomes invisible by visual masking techniques as controlled in the direct prime recognition task. Typically, the prime fits exactly into an inner cut-out in the target yielding metacontrast masking of the prime (Klotz, \& Neumann, 1999; Neumann, \& Klotz, 1994). Subliminal prime perception but measurable behavioral outcomes satisfy the criteria for a simple dissociation. In contrast, however, to the neurological disorders mentioned above, the response priming paradigm allows to achieve a double dissociation by varying the temporal interval of prime and target presentation which can have opposite effects on prime visibility and priming effects (Lau, \& Passingham, 2007; Mattler, 2003; Vorberg, et al., 2003). Increasing the stimulus onset asynchrony (SOA) leads to a corresponding increase in priming effects which means larger performance benefits on congruent and larger performance costs on incongruent trials. The direct measure of prime visibility also changes as a function of SOA. This masking function depends on various parameters of stimulus presentation (Breitmeyer, \& Ögmen, 2006) and is subject to interindividual variability (Albrecht, Klapötke, \& Mattler, 2010). Nevertheless, with metacontrast masking a set of conditions can be established that yield deteriorating recognition performance with increasing SOA which is termed Type-B masking (Breitmeyer, 1984). Then, prime perception is largely unimpaired at shortest SOAs and then decreases with increasing SOAs and is lowest at some intermediate SOAs (30 - $100 \mathrm{~ms}$, Breitmeyer, 1984) before recognition performance again recovers. Thus, across a substantial range of SOAs, decreasing prime visibility is accompanied by increasing indirect effects of the prime which implies that both, prime visibility and its behavioral effects, rely on different underlying processes, and thus both are independent (Schmidt, \& Vorberg, 2006).

Taken together, response priming is a suitable experimental tool to demonstrate the existence of subliminal stimulus processing. During the last decade, the phenomenon has received considerable attention and the influence of subliminal primes has been extended to higherorder processes, like in semantic priming (Bodner \& Masson, 2003; Pohl, Kiesel, Kunde \& Hoffmann, 2010; for a meta-analysis see: Van den Bussche, van den Noortgate, \& Reynvoet, 2009) or priming of cognitive operations (Mattler, 2003). However, the boundary conditions are still a matter of debate (e.g. Kiefer, \& Martens, 2010; for a review see Kouider, \& Dehaene, 2007). As part of the diverse literature on subliminal priming, a surprising reversal of priming effects has been discovered which has become known as the inverse priming effect or negative compatibility effect (Eimer, \& Schlaghecken, 1998). The theoretical and empirical 
work presented in this thesis is meant to examine this inverse priming effect and the mechanism underlying it. To this end, the first chapter presents a brief review of the existing literature on inverse priming. First, the conditions for inverse priming to occur are described and the most important theoretical accounts will be outlined roughly in chronological order. The accounts will also be classified according to which origin they ascribe to the inverse priming effect. Next, evidence for the respective theories will be discussed. Finally, the role of stimulus presentation parameters on the generation of inverse priming is discussed especially focusing on the design of the stimuli involved. The second chapter constitutes the empirical part including five experimental projects performed on inverse priming. These projects are already published or prepared for publication. The respective manuscripts can be found in the Appendix. In the third chapter, the findings will be integrated and discussed along with previous results from the literature attempting to integrate the findings into a common taxonomy of inverse priming effects. To anticipate the conclusions, inverse priming should be understood as a diverse phenomenon resulting from multiple sources dependent on several experimental parameters. Therefore, experimental findings should be carefully interpreted and considered with respect to the experimental situation. Especially, the design of the stimuli might be crucial for how the effect is generated.

\subsection{Inverse Priming}

Eimer and Schlaghecken (1998) intended to study response priming. In contrast to previous studies, in which the target served as a backward masking stimulus for the prime at the same time (e.g. Neumann, \& Klotz, 1994), they employed a three-stimulus paradigm including a separate masking stimulus following the prime but preceding the target stimulus. Primes and targets were double arrows which pointed either to the left $(<<)$ or to the right $(>>)$. In the congruent condition both stimuli, primes and targets, were identical and in the incongruent condition the arrows were of opposite orientations. On half of all trials, a neutral prime was presented $(<>$ or $><$ ). The intervening masking stimulus consisted of the superposition of both stimulus alternatives. In the indirect task, subjects were required to report the orientation of the target stimulus as fast and accurately as possible. Opposite to their predictions, Eimer and Schlaghecken (1998) found target classification performance to be better on incongruent trials as compared to congruent trials. Relative to the neutral baseline condition, performance costs were observed when a congruent prime preceded the target and performance benefits with preceding incongruent primes. This counterintuitive inverse priming effect occurred even 
though the prime was rendered almost perfectly invisible by masking as indexed by chancelevel performance on a separate direct task which involved reporting the identity of the prime. Since the original work by Eimer and Schlaghecken, the inverse priming effect has been replicated in numerous studies (e.g. Eimer, 1999; Lleras, \& Enns, 2004; Klapp, \& Hinkley, 2002; Mattler, 2005; 2006; 2007; Sumner, 2008; Verleger, Jaśkowski, Aydemir, van der Lubbe, \& Groen, 2004).

During the past decade various attempts have been made to explain the inverse priming effect. The existing accounts differ with respect to the assumed source of the effect within the information processing stream from the perceptual analysis of the stimulus material to the execution of a motor response. Traditionally, at least three consecutive levels are differentiated along this visuomotor translation path (McClelland, 1979; Sanders, 1980; Smith, 1968; Sternberg, 1969). Visual stimuli are first detected and identified on the perceptual level. On the subsequent central level of processing, the perceptual representation is mapped onto a corresponding abstract response code dependent on the current stimulusresponse (SR) association rules. Finally, the appropriate motor response is selected and initiated on the motor level. Some accounts postulated a perceptual locus (Huber, 2008; Sohrabi, \& West, 2009), others a central locus (Cole, \& Kuhn, 2010; Klauer, \& Dittrich, 2010) and further researchers adhere to a motor locus of the inverse priming effect (Jaśkowski, \& Przekoracka-Krawczyk, 2005; Lleras, \& Enns, 2004, 2006; Schlaghecken, \& Eimer, 2002). In the following, the seven most influential theoretical accounts will be briefly presented along with the most important empirical findings on inverse priming motivating these accounts. First of all, motor accounts will be discussed because during the first years the theoretical work on inverse priming concentrated on a motor origin of the effect. Later on, several more recent accounts advocated either a perceptual or a central origin.

\subsection{Motor accounts of inverse priming}

Motor self inhibition. The first hypothesis on inverse priming was the motor self-inhibition account (Schlaghecken, \& Eimer, 2002; Schlaghecken, Bowman, \& Eimer, 2006). The core assumption was that inverse priming reflects an automatic, inhibitory mechanism located within the motor system serving to counteract the initial prime-evoked response activation. The account was inspired by the demonstration of the time-course of priming effects in the inverse priming paradigm. At short mask-target SOAs, positive priming effects with benefits for prime-congruent targets were observed which reversed at longer mask-target SOAs such 
that performance on incongruent trials was better (Mattler, 2007; Schlaghecken, \& Eimer, 2000; Schlaghecken, \& Maylor, 2005; Schlaghecken, Birak, \& Maylor, in press; Sumner, \& Brandwood, 2008). This facilitation-followed-by-inhibition time course was also reflected in electrophysiological data (lateralized readiness potentials, LRP, Eimer, \& Schlaghecken, 1998, 2003; Praamstra, \& Seiss, 2005). The reversal of prime-induced response activation was interpreted as a developing self-inhibition of the primed response and dis-inhibition of the alternative response. A close link between conscious prime perception and the reversal of priming effects (Eimer, \& Schlaghecken, 2002; Klapp, \& Hinkley, 2002) suggested that automatic self-inhibition is triggered by the removal of perceptual evidence for the prime due to masking. Later on, the additional assumption of a minimum sensory strength of the prime to trigger the inhibitory mechanism was added (Schlaghecken, \& Eimer, 2002). This idea was motivated because weakening the perceptual strength of the prime elicited only positive, but not inverse priming. For instance, peripheral primes (Schlaghecken, \& Eimer, 2000) or primes whose perceptual salience was reduced by embedding them within a random dot pattern (Schlaghecken, and Eimer, 2002) failed to produce inverse priming. To summarize the account, a prime of sufficient perceptual strength activates its assigned response and due to masking this preliminary activation becomes inhibited leading to inverse priming if this timeconsuming process could develop until target presentation with sufficiently long mask-target SOAs.

Mask triggered inhibition. In the following, demonstrations of inverse priming with completely unmasked primes casted serious doubts on this original version of the selfinhibition account. In contrast to previous results (Eimer, \& Schlaghecken, 2002) inverse priming also occurred with primes presented about $2^{\circ}$ above and below fixation while "masks" were placed at fixation (Jaśkowski, Biłuńska, Tomanek, \& Verleger, 2008). Thus, "masks" did not reduce prime visibility at all but rather act like a distracter and still inverse priming occured. Inverse priming with such non-masking flankers has been repeatedly demonstrated (Lleras, \& Enns, 2006; Jaśkowski, 2007; 2008). Furthermore, Schlaghecken and colleagues (2008) found a dissociation of priming effects from visibility in a perceptual learning experiment. While prime visibility continuously improved across five learning sessions, priming effects remained the same. These results brought them to relax their claim of a close causal link between prime visibility and priming effects (Schlaghecken, et al., 2008). Along the same lines, others advocated a more general inhibitory mechanism triggered by the mask irrespective of prime visibility (Jaśkowski, \& Przekoracka-Krawczyk, 2005; Mattler, 2005; Lleras, \& Enns, 2006). Thus, the only difference of these mask-triggered 
inhibition accounts to the self-inhibition account is whether the primed response inhibits itself or the inhibition is stimulus-triggered by the mask (Sumner, 2007; Wilson, Tresilian, \& Schlaghecken, 2010). In other words: Not the removal of the prime evidence itself by the mask is critical, but the occurrence of another potentially relevant visual stimulus causes inhibition of any on-going motor activation. Therefore, inhibition is especially effective when the masking stimulus contains task-relevant features (Jaśkowski, \& Verleger, 2007).

Object Updating. The idea of an inhibitory mechanism was challenged by some researchers underscoring the special design of masking stimuli in many early studies on inverse priming (Lleras, \& Enns, 2004; Verleger, et al. 2004). Typically, these early studies employed masks simply built by superimposing the prime alternatives upon one another (e.g. Eimer, 1999; Eimer, \& Schlaghecken, 1998; Eimer, Schubö, \& Schlaghecken, 2002; Schlaghecken, \& Eimer, 2000; 2004; Schlaghecken, Münchau, Bloem, Rothwell, \& Eimer, 2003). Within the framework of their object updating theory, Lleras and Enns (2004) argued that the rapid successive stimulus presentation in the inverse priming paradigm leads to the formation of a common object representation which is iteratively updated each time the visual input changes. Novel elements become especially salient due to a novelty bias in perceptual processing implying that those mask elements, which are not part of the prime, gain a saliency advantage. In case of the superposition mask, this perceptual interaction leads to a salience bias in favor of the prime-opposite object representation. This current perceptual state is directly translated to the motor system. Thus, even though priming is inverse with respect to the prime, it is in fact a positive priming effect because new features in the mask activate the prime-incongruent response. Consequently, object updating explains inverse priming without the need to assume an inhibitory mechanism.

The Object Updating account is capable of explaining inverse priming with so-called relevant masks - masks that contain task-relevant features like the superposition mask. It is now widely accepted that with such relevant masks the perceptual interaction of prime and mask at least contributes to inverse priming (Jaśkowski, \& Przekoracka-Krawczyk, 2005; Klapp, 2005; Klauer, \& Dittrich, 2010; Kiesel, Berner, \& Kunde, 2008; Mattler, 2005; Schlaghecken, Rowley, Sembi, Simmons, \& Whitcombs, 2007; Sumner, 2007). The role of object updating receives further support from the finding that inverse priming effects with relevant masks are typically much larger compared to inverse priming with irrelevant masks. Such irrelevant masks are completely free of task-relevant features like for instance a grid of vertical and horizontal lines for the case of arrow-shaped prime and target stimuli (Jaśkowski, 2007, 2008; 
Jaśkowski, et al., 2008; Jaśkowski \& Przekoracka-Krawczyk, 2005; Kiesel, et al., 2008; Lleras \& Enns, 2004, 2005, 2006; Schlaghecken \& Eimer, 2006; Verleger, Görgen, \& Jaśkowski, 2005; Verleger et al., 2004).

Importantly, however, Object Updating is incapable of covering all instances of inverse priming because inverse priming has been repeatedly demonstrated with irrelevant masks completely free of task-relevant features (Eimer \& Schlaghecken, 2002; Klapp, 2005; Klapp \& Haas, 2005; Lleras \& Enns, 2005; Schlaghecken \& Eimer, 2006; Schlaghecken et al., 2007; Sumner, 2008). Inverse priming with irrelevant masks can be explained by the above mentioned accounts proposing an inhibitory mechanism located in the motor system (Jaśkowski, \& Przekoracka-Krawczyk, 2005; Lleras, \& Enns, 2006; Schlaghecken, \& Eimer, 2002). In opposition to this idea, some more recent accounts proposed different mechanisms operating at a perceptual or a central level.

\subsection{Perceptual Accounts of Inverse Priming}

In contrast to the aforementioned ideas, one commonality across perceptual (but also central) accounts is that the masking stimulus is considered to be inessential for inverse priming. The motor accounts either conceptualize the mask as the trigger for inhibition of the primed response or by means of its perceptual interaction with the prime as a trigger for the activation of the unprimed response. Consequently, the masking stimulus is considered to cause the processes leading to inverse priming (cf. Boy, Clarke, \& Sumne, 2008; Lleras, \& Enns, 2006). In contrast, perceptual accounts assume that perceptual processing of a prime-incongruent target is facilitated relative to a congruent target either because of habituation (Huber, 2008) or a conflict-sensitive attentional refractory period (Sohrabi, \& West, 2009). The activationfollowed-by-inhibition time-course of priming effects is interpreted as the result of varying prime-target delays regardless of the temporal onset of the mask within the prime-target interval.

Habituation. Huber (2008) presented a neural network model inspired by his research on word identification processes. From this perspective, the positive effect at short mask-target SOAs (Schlaghecken, \& Eimer, 2000), when target presentation is just slightly delayed relative to the prime, is due to source confusion: the visual system erroneously confounds prime-evoked perceptual activation with target activation. Thus, perceptual processing of congruent targets is facilitated relative to incongruent targets leading to positive priming effects. However, participants are required to respond only to the target. Therefore, prime 
activity is discounted within the visual system by means of habituation to reduce source confusion. Habituation is assumed to affect target processing only at longer prime-target delays. It reduces the sensitivity of stimuli the visual system has just been exposed to before, thus, slowing the perceptual processing of congruent targets which translates to the observed behavioral performance costs on congruent trials at long SOAs.

Attentional refractory period. A different perceptual account was proposed by Sohrabi and West (2009). According this view, inverse priming results from an attentional modulation of the perceptual processing of the target. On congruent trials, attention is biphasic and depends on prime-target SOA. Processing of congruent targets is thought to be facilitated at short prime-target SOAs yielding positive priming effects. In contrast, as prime-target SOA increases, attention collapses due to an attentional refractory period deteriorating target processing (similar to the attentional blink, see Martens, \& Wyble, 2010). On incongruent trials, however, the divergent prime and target information induces a conflict which reduces the effect of the attentional refractory period. Thus, perceptual processing of incongruent targets is less strongly impaired compared to congruent targets leading to inverse priming at longer prime-target SOAs.

\subsection{Central Accounts of Inverse Priming}

In addition to motor accounts and perceptual accounts, recently two further accounts have been presented which ascribed the inverse priming effect to the level of abstract response selection.

Spatial attention. Cole and Kuhn (2010) proposed an account inspired by the fact that almost all studies on inverse priming employed arrows as prime and target stimuli. It is well known from the literature on spatial cueing that arrows lead to an automatic, involuntary shift of spatial attention even if it is detrimental in the current task context (counterpredictive cues, Tipples, 2008; Kuhn, \& Kingstone, 2009). In a series of experiments, Cole and Kuhn (2010) first establish that arrow primes in the inverse priming paradigm do indeed induce such a lateral shift of attention according to their orientation, thus, away from the subsequent (centrally presented) target. To identify the target, attention has to be redirected to the center requiring a backward shift of attention in the opposite direction. Cole and Kuhn (2010) demonstrated that the direction of this backward movement interferes with the response selection for a central arrow target. In inverse priming, the direction of this latter attentional 
shift matches the target orientation on incongruent trials but conflicts with it on congruent trials. For instance, if the prime points to the left, attention is first shifted to the left, returns to the center (attentional shift to the right) which facilitates response selection of a right response, i.e. the correct response for an incongruent target. Many studies on inverse priming employed arrow stimuli but not all (Jaśkowski, \& Ślósarek, 2007; Schlaghecken, et al., 2007; Verleger, et al., 2005). For instance, Jaśkowski and Ślósarek (2007) demonstrated inverse priming with double rectangular brackts ([[ vs. ]]) as primes and targets. Similar to arrows, these stimuli are also spatially compatible to the responses. However, whether such non-arrow stimuli also elicit automatic attentional shifts remains unclear (cf. Guzzon, Brignani, Miniussi, \& Marzi, 2010). Consequently, the explanatory power of the spatial attention account for inverse priming might be limited to the use of arrow stimuli.

Evaluation window account. The evaluation window account is another recent example which locates inverse priming at central levels of processing (Klauer, \& Dittrich, 2010). From this perspective, inverse priming occurs whenever the prime is excluded from a temporally limited evaluation window during which evidence for the responses is gathered. According to Klauer and Dittrich, two evidence accumulators which represent the two response alternatives are fed by incoming perceptual evidence. The decision for one or the other response is based upon the relative increase across a certain time period, the evaluation window, which is positioned dependent on the current task structure. Positive priming results whenever the prime falls into the evaluation window because then the increase is higher on congruent trials. In contrast, when the onset of the evaluation window is set after prime presentation, thus excluding the prime, the relative increase is higher on incongruent trials, which in turn leads to inverse priming. This is because the prime, even if excluded from the window, biases the initial state of the accumulators and increase rates depend on the absolute state of the accumulator. As postulated by the Weber-Fechner law (e.g. Miller, 1964), the increase rate declines with increasing counter state. Therefore, the unbiased counter, which represents the unprimed response, shows larger increases across the evaluation window while the primed counter shows less increase following the same stimulation. In inverse priming, the temporal positioning of the evaluation window is likely to exclude the prime because the mask reliably announces target presentation making the mask an ideal go-signal for the evaluation process. In contrast, the prime could serve as such a temporal marker in the positive priming domain leading to inclusive evaluation windows because it is immediately followed by the target stimulus. Taken together, the evaluation window accounts predicts inverse priming because the increase across the evaluation process (triggerd by mask onset) is larger if the accumulator 
for the target response is initially unbiased (incongruent prime) compared to when it already reached some non-zero state (congruent prime).

To sum up, various attempts have been made to explain the inverse priming effect. Among these accounts, Object Updating has a special status for two reasons. First, it is focused on the use of special masks which include task-relevant features, while all other accounts explain inverse priming irrespective of the design of the mask. Second, Object Updating explains inverse priming in terms of an active support for the prime-incongruent response (which results from the perceptual prime-mask interaction) while the other accounts emphasize impaired processing of congruent targets. This impairment is considered to result either from inhibition of the primed response due to masking (Schlaghecken, \& Eimer, 2002) or simply due to the presentation of a new stimulus (Jaśkowski, \& Przekoracka-Krawczyk, 2005, Lleras, \& Enns, 2006; Mattler, 2005) or from hampered perceptual processing as a result of habituation (Huber, 2008) or an attentional refractory period (Sohrabi, \& West, 2009). Furthermore, selection of the congruent response could be impaired due to interferences induced by attentional movements (Cole, \& Kuhn, 2010) or a bias towards the primed response at the start of the evaluation accumulation process which implies larger increase rates for the unprimed response facilitating its selection (Klauer, \& Dittrich, 2010). The importance of Object Updating to account for inverse priming with relevant masks seems to be beyond controversy (Jaśkowski, \& Przekoracka-Krawczyk, 2005; Klapp, 2005; Klauer, \& Dittrich, 2010; Kiesel, et al., 2008; Mattler, 2005; Schlaghecken, et al., 2007; Sumner, 2007). With irrelevant masks, however, all other accounts claim to account for the inverse priming effect. To disentangle possible contributions of the accounts several empirical studies have been performed to localize inverse priming effects in the information processing stream.

\subsection{The locus of inverse priming effects}

The various theoretical accounts on inverse priming stimulated a large amount of empirical research. The adherents of a motor account of inverse priming underline their view with behavioral and physiological data. An early experimental paradigm suitable to isolate motor components of the inverse priming effect has been developed by Eimer (1999). Eimer extended the standard paradigm by intermixing arrow targets with other, perceptually dissimilar targets presented laterally on the screen. From the perspective of a perceptual locus (e.g. Huber, 2008), inverse priming effects to such lateral targets should be completely absent because the repeated presentation of physically identical (or at least similar) stimuli yields the 
effect. To the extent that inverse priming effects evoked by cental arrow primes translate to such lateral targets post-perceptual processes should be involved in the generation of inverse priming. In different studies, priming effects fully transferred to lateral stimuli suggesting a motor locus of the effect (Eimer, 1999; Klapp, \& Hinkley, 2002). Schlaghecken and Eimer (2000) also replicated this finding when taking into account the overall faster response speed to such lateral targets.

These results are supplemented by further behavioral studies demonstrating inverse priming on free-choice responses (Klapp, \& Haas, 2005; Klapp, \& Hinkley, 2002; O’Connor, \& Neill, 2011; Schlaghecken, \& Eimer, 2004; Schlaghecken, Klapp, \& Maylor, 2009). In the freechoice paradigm, masked primes were either followed by the typical arrow target or a neutral stimulus (e.g. an annulus, Schlaghecken, et al., 2009) which allowed the subject to freely choose one response alternative. Even though participants believed to freely choose the response in these cases, the response alternative opposite to the masked prime was more frequently chosen which would be readily explained by a motor theory of inverse priming but which is hard to account for by a perceptual theory. The motor inhibition accounts predict inverse priming on free-choice responses because primes affect motor processes and response inhibition yields an advantage for the prime-incongruent response. In contrast, perceptual accounts would not predict inverse priming with neutal free-choice target stimuli because they are equally similar to both primes, and thus, perceptual processing is the same irrespective of the prime.

Moreover, Boy and Sumner (2010) demonstrated a complete, but transient reversal of priming effects following an unexpected reversal of arbitrarily chosen SR-mappings (inverse priming effects became suddenly positive). After such a reversal, a perceptual account would not predict any change in priming effects because the visual similarity is not affected by such a change: congruent primes and targets are still perceptually similar and therefore the perceptual analysis of the target should be still hampered after a reversal. In contrast, motor theories predict the reversal of priming effects because the disinhibited (unprimed) response is the correct one for a congruent target after the SR-mapping is established. In concrete terms, before the reversal occurred, a prime which e.g. activates the left response would facilitate execution of the right response due to motor inhibition. After the reversal, however, such a right-hand response is appropriate for the congruent target leading to better performance for such congruent targets and thus positive priming. 
This behavioral evidence for a motor locus is in line with corresponding physiological findings. Electrophysiological research demonstrates that inverse priming is reflected by corresponding response preparation processes indexed by the LRP (Eimer, \& Schlaghecken, 1998, 2003; Praamstra, \& Seiss, 2005). At first glance, such motor activations might suggest a motor locus of inverse priming. However, one has to bear in mind that physiological effects within the motor system could readily be the consequence of earlier, e.g. perceptual, processes that are directly linked to corresponding motor preparation processes (Lleras, \& Enns, 2004). In contrast, more recent physiological data demonstrates a central role of the supplementary motor area in inverse priming (SMA, Boy, Evans, et al., 2010; Boy, Husain, et al., 2010; Sumner, et al., 2007). Sumner and colleagues (2007), for instance, presented a patient with a focal lesion within the SMA who failed to show inverse priming while adequate control subjects showed the effect suggesting that inverse priming results from processes in the motor system. The SMA is a cortical area situated within the dorsomedial part of the frontal cortex. It is crucially involved in motor control processes (for a review: Nachev, Kennard, \& Husain, 2008).

Further studies were designed to narrow down the origin of inverse priming within the motor system. Schlaghecken and colleagues (2003) applied repetitive transcranial magnetic stimulation (rTMS) to modulate excitability within the primary motor cortex. While rTMS produced an overall slowing of responses, inverse priming effects were unaffected suggesting that inverse priming does not originate from the primary motor cortex. Additionally, Schlaghecken, Klapp and Maylor (2009) failed to observe an inverse priming effect on bimanual responses whereas such an effect was present on freely chosen responses or on the frequency of erroneously executed responses in a NoGo-task. If primes would be capable to control individual muscle commands, the hand opposite to the prime direction should lead bimanual response execution as observed before for the Simon task: the hand ipsilateral to the target stimulus responded slightly faster when bimanual responses were required (Miller, \& Franz, 2005). Taken together, these results provide evidence for the view that inverse priming does not originate from primary motor cortex.

In opposition to these experimental findings favoring a motor locus, other results support a perceptual origin of inverse priming. Mattler (2006) examined the role of visual similarity between prime and target stimuli in inverse priming. Performance for target stimuli that were either congruent and perceptually similar to the primes, congruent and dissimilar or incongruent was compared. Motor influences should produce inverse priming irrespective of 
visual similarity, thus, yielding better performance on incongruent as compared to congruent and dissimilar primes. However, to the extent that perceptual processes are involved performance should suffer for congruent and similar primes. Behavioral results for congruentdissimilar and incongruent primes were indistinguishable, only similar primes produced the expected performance costs suggesting that entire effect is due to perceptual processes. In addition, inverse priming has been shown to extend to more complex non-motor operations which seems to obey to the same laws as inverse motor priming (Mattler, 2007; see also: Mattler, 2003). In such a non-motor priming paradigm, primes do not possess any predictive value for the upcoming response. Instead, primes are associated with a certain cognitive operation. Consequently, primes cannot affect motor processes.

Thus, at first glance empirical findings seem to be contradictory. Several studies support a post-perceptual locus while other findings point to perceptual source of the inverse priming effect. To resolve this contradiction a taxonomy of inverse priming effects is proposed in this thesis based on the design of the stimuli involved. Previous work on the role of stimulus structure in inverse priming is presented in the following section.

\subsection{A taxonomy of inverse priming based on stimulus structure}

The discovery of the phenomenon of inverse priming in 1998 produced a broad range of theoretical explanations along with a rich but often seemingly contradictory empirical literature. Notably, inverse priming effects largely varied across different studies even though the same sequence of three stimuli, prime, mask and target, with comparable timing parameters has been used. While Eimer and Schlaghecken (1998) observed about $50 \mathrm{~ms}$ response time (RT) advantage on incongruent trials when they originally discovered the phenomenon, the effect decreased to $10-20 \mathrm{~ms}$ in a later work (Schlaghecken, \& Eimer, 2006) or was even completely absent (Jaśkowski, \& Ślósarek, 2007). The current thesis is meant to provide an answer to the question of which factors are responsible for the astonishing variability of inverse priming effects even though the experimental procedure is virtually the same. This thesis will advocate the view that the design of the stimuli is one crucial factor for inverse priming. The varying size of effects provides a first clue that the mechanisms underlying the effect might differ implicating that the same observable outcome, the inverse priming effect, is generated in different ways dependent on the structure of the stimuli involved. Thus, the inconsistencies in theoretical accounts and the divergent empirical results are the product of neglecting the specific design of the stimuli employed in the respective 
experiments. In the inverse priming paradigm, primes, masks and targets can be distinguished according to two important characteristics.

The first crucial distinction is whether the masking stimulus comprises task-relevant features or not. Eimer and Schlaghecken (1998) employed relevant masks which produced large effects compared to the use of an irrelevant mask (Schlaghecken, \& Eimer, 2006). This effect of the mask structure on the magnitude of inverse priming effects can be explained by Object Updating. Mask relevance is certainly a crucial factor explaining variability of inverse priming effects among different studies but it does not fully capture all instances because Jaśkowski and Ślósarek (2007) even failed to find any inverse priming effect employing an irrelevant mask. However, in contrast to Schlaghecken and Eimer (2006), they used prime (and target) stimuli which were not compatible to the response set. Almost all studies on inverse priming, used double arrow stimuli whose orientation had to be reported by pressing a spatially corresponding response button $(\ll<$ required a left-hand response, $>$ a right-hand response). Thus, stimuli and responses varied on a common spatial dimension, a situation which had been termed dimensional overlap of stimuli and responses (Kornblum, et al., 1990). Then, stimuli might automatically activate the corresponding response (Eimer; 1993; 1995; Kornblum, et al., 1990). Instead of double arrows, Jaśkowski and Ślósarek (2007) adopted spatially non-compatible geometric figures (a square and a diamond) which are symmetrical along the horizontal dimension along which responses are aligned. Such stimuli are arbitrarily mapped onto the responses. Then, response preparation is supposed to result from a controlled response selection process based on previously established SR-associations (Kornblum, et al., 1990; Proctur, Lu, Wang, \& Dutta, 1995). As suggested by the data reported by Jaśkowski and Ślósarek (2007), response compatibility ${ }^{1}$ is a second crucial factor modulating inverse priming effects.

Consequently, four distinct stimulus categories could be discerned in the study of inverse priming. These categories result from orthogonal crossing of the response compatibility of the primes and targets and the relevance of the mask which is illustrated in Figure 1. The varying size of inverse priming within these categories might be taken as preliminary evidence for the involvement of different mechanisms producing the effect within each category (alternatively, the stimulus structure could modulate the effectiveness of a single mechanism). To further

\footnotetext{
${ }^{1}$ Throughout the current thesis, the term response compatibility will be used. However, response compatibility is not meant to be restricted to motor priming. In close correspondence to SR-compatibility in motor tasks, in non-motor priming (Mattler, 2007), a compatible mapping would involve that prime and cue stimuli and the two alternative tasks vary along a common dimension.
} 
characterize how inverse priming is generated within each stimulus category, five separate research projects have been accomplished, each of which consisting of a series of single experiments. Every single project applied a different experimental paradigm and used different stimuli in order to derive conclusions about how the effect had been produced. The stimulus categories studied in the respective projects are also summarized in Figure 1.

In Project I, we intended to localize inverse priming with relevant metacontrast masks and non-compatible stimuli based on previous research with the same stimulus material (Mattler, 2007). Since results might not be valid for other stimulus material, we sought to construct an irrelevant mask. To do so, we tried to isolate the distinguishing feature of relevant and irrelevant masks in Project II. In Project III, we used a purely irrelevant mask and studied where and how inverse priming is generated with response-compatible and non-compatible stimuli. Because we obtained evidence for a motor locus with the stimuli employed, inverse priming should not occur in a non-motor cue priming paradigm. This hypothesis was explored in Project IV. The different findings for the functional localization of inverse priming with the

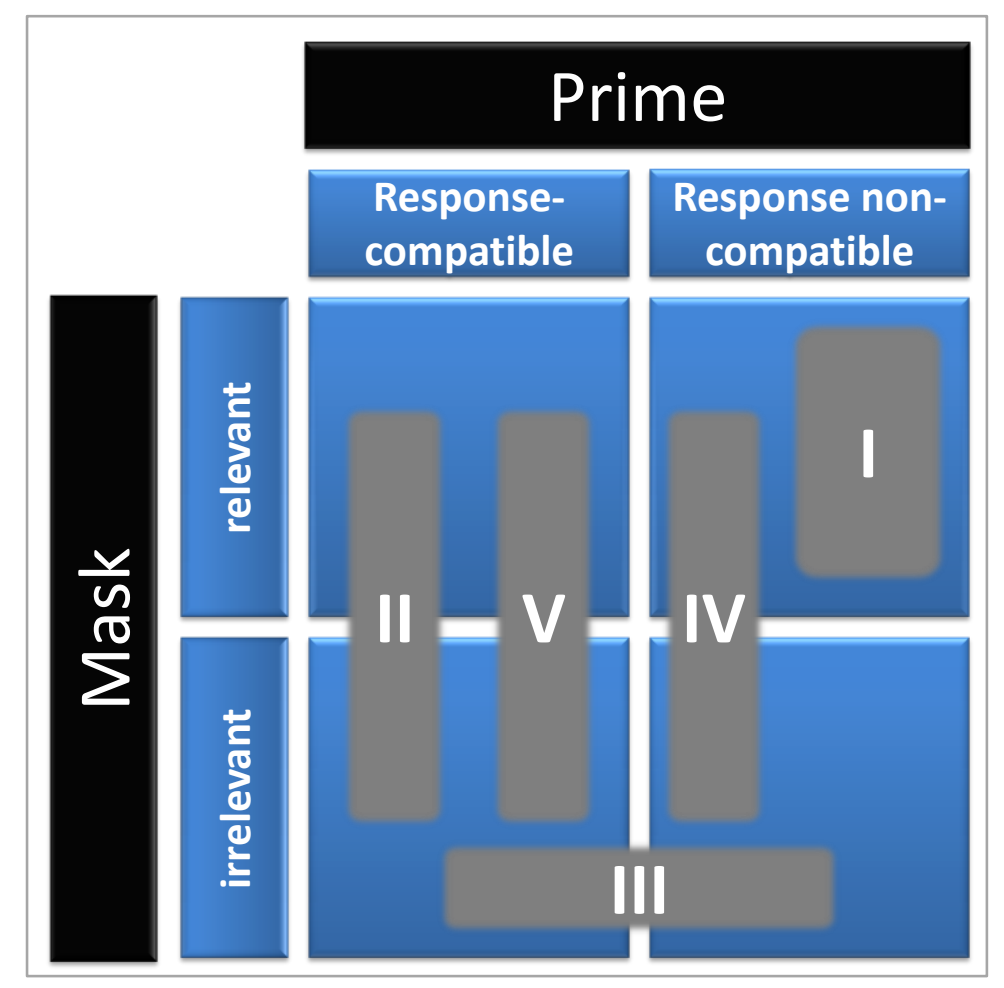

Figure 1. Illustration of the stimulus dimensions determining inverse priming effects. Grey boxes represent the five projects referred to by the latin numbers. The areas the boxes are covering represent the stimulus dimensions investigated in the respective project. For example, Project I addresses inverse priming with response noncompatible stimuli and relevant masks. In contrast, Project II combined response-compatible stimuli with both, relevant and irrelevant masks. 
different stimuli are then integrated and tested in the final Project V which applies functional magnetic resonance imaging in order to examine the anatomical substrate underlying the mechanism which produces inverse priming with relevant masks on the one hand and with irrelevant masks on the other hand. 


\section{Empirical part}

Five separate projects were conducted to address the mechanisms underlying inverse priming. The experimental paradigm and the stimuli employed differ across the projects allowing us to disentangle the diverse contributions to the inverse priming effects dependent on the stimuli used. The experimental design along with the most important findings will be briefly presented in the following chapter. Details can be found in the respective manuscripts (see Appendix I to V).

\subsection{Project I: PRP-paradigm}

In a first attempt to localize inverse priming effects, we applied the Psychological Refractory Period (PRP) paradigm (Krüger, Klapötke, \& Mattler, 2011, Appendix I). To this end, the inverse priming task was combined with a secondary tone discrimination task. Two complementary variants of the PRP-paradigm were used in two different experiments which were identical apart from the temporal order in which the two tasks had to be performed. In Experiment 1 we employed the Effect-Absorption procedure, in which the secondary tone task had to be performed briefly before the visual inverse priming task. In Experiment 2 the Effect-Propagation procedure was applied such that the visual task had to be performed first. Importantly, we adopted a version of the inverse priming task which had been successfully studied before (Mattler, 2007). Primes and targets were simple visual shapes, namely squares and diamonds which were thus response non-compatible. Primes were followed by a neutral, star-shaped metacontrast mask which possessed an inner cut-out which is identical to the overlay of both prime variants. Therefore, the mask can be considered as a relevant mask. Those timing parameters yielding largest inverse priming effects in a prior study (Mattler, 2007) were chosen (cf. Figure 3 from Krüger, et al., 2011; Appendix I).

To understand why the PRP-paradigm is suitable to localize the inverse priming effect one has to take into account two core assumptions. First, the paradigm is based on the three mentioned consecutive information processing levels: perceptual analysis of visual input, central mapping of this input onto a motor response and execution of that response at the motor level. Inverse priming could then arise from processing on any of these three levels. Second, processing on the central level is conceptualized as a structural bottleneck (Pashler, \& Johnston, 1998; Ullrich, \& Miller, 2008). While processing on all other levels can run in parallel, resources are limited on the central level allowing only one task to be processed at a 
time. Whenever two tasks have to be performed in close temporal succession, central response selection for the second task has to wait until central processing of the first task is completed.

The PRP-paradigm allows localization of experimental effects like the inverse priming effect because the temporal distance between the two tasks is varied. At short SOA $(50 \mathrm{~ms})$, interference among the two tasks occurs which is assumed to result from the processing bottleneck. The pattern of these interference effects allows the localization of the inverse priming effect relative to the central bottleneck. In the Effect-Absorption Procedure (Experiment 1), subjects first had to discriminate the pitch of a tone before accomplishing the visual inverse priming task. At short SOA, response selection for the tone task is not fully accomplished when processing in the visual task reaches the bottleneck. Thus, the visual task has to wait. If the inverse priming effect originates from processing on perceptual levels it would not become manifest in the performance due to this waiting period (cf. Figure 1 from Krüger, et al., 2010; Appendix I). If on the other hand, (parts of) of the inverse priming effect result from processing on central or motor levels a (residual) inverse priming effect should emerge. As a reference condition, a long SOA $(800 \mathrm{~ms})$ is introduced which allows for sequential and therefore independent performance of the two tasks. Consequently, any reduction of the inverse priming effect at short SOA as compared to long SOA would be attributable to a perceptual source of the effect.

In the Effect-Propagation Procedure (Experiment 2), the visual inverse priming task has to be performed first and at short SOA $(50 \mathrm{~ms})$, response selection for the secondary tone discrimination task has to wait. If the inverse priming effect affects processing time at perceptual or central levels, the total duration of this waiting period is also affected by primetarget congruency. Therefore, processing on the secondary task would start earlier in the incongruent condition of the visual task compared to the congruent condition which in turn would also lead to overall faster responses on incongruent trials for the auditory task. Consequently, the inverse priming effect would then propagate to the tone task (cf. Figure 2 from Krüger, et al., 2010, Appendix I). However, if priming effects arise from motor levels of processing no such transfer would be expected because then congruency would matter after the waiting period not affecting the secondary task. Again, a long interference-free SOA condition is part of the experimental design providing a reference for the inverse priming effect.

We obtained clear-cut results from the two experiments favoring a perceptual locus of the inverse priming effect. In Experiment 1, inverse priming effects completely disappeared at 
short SOA. Following the logic of the PRP-paradigm, this finding indexes an absorption of the effect into the waiting period which implies a perceptual locus. This result is complemented by independent evidence from Experiment 2. Inverse priming was of a comparable magnitude, thus, fully propagated to the secondary tone task pointing to a perceptual or central locus of the inverse priming effect. Taken together, these results suggest a perceptual source of the inverse priming effect. Thus, results corroborate previous data isolating the effect of visual similarity in inverse priming (Mattler, 2006). Furthermore, the existence of inverse non-motor priming (Mattler, 2006; 2007) casts doubts on the assumption of a motor locus but is in line with a perceptual explanation of inverse priming.

However, the validity of the results could be limited to the current experimental situation because of the special design of the stimuli used. The metacontrast-mask is built by superimposing the two stimulus alternatives. It is unclear whether inverse priming with such a relevant mask constitutes a special case. If inverse priming with relevant and irrelevant masks indeed results from completely distinct mechanisms (Separate-Mechanisms Approach, Krüger, et al., 2011), the generalizability of our results is restricted to the use of relevant masks. If, on the other hand, inverse priming with both types of mask relies on a shared mechanism, our evidence for a perceptual locus would be valid for irrelevant masks, too. A shared mechanism could either be a single mechanism whose efficiency is modulated by mask relevance, i.e. it produces larger effects with relevant masks (Single-Mechanism Approach) or a basic mechanism generates the entire effect for irrelevant masks while an additional mechanism operates only with relevant masks producing the larger observable inverse priming effects (Co-Active Mechanisms Approach).

Krüger et al. (2010) argued in favor of the Co-active Mechanisms Approach. From this point of view, inverse priming should originate from a perceptual source with both, relevant as well as irrelevant masks. The perceptual accounts proposed by Huber (2008) and Sohrabi and West (2009) would readily explain the shared component across the two masks. Due to this view, inverse priming results from hampered perceptual processing of targets preceded by a congruent prime irrespective of mask structure. This basic mechanism might be supplemented by an additional mechanism which is only effective with relevant masks. As proposed by Lleras and Enns (2004), the saliency advantage for prime-incongruent mask features might facilitate processing of an incongruent target. However, in contrast to their explanation it is not the activation of the unprimed response which follows from the saliency bias (Lleras, \& Enns, 2004). Instead, we proposed that the activation of the unprimed object representation 
facilitates recognition of a corresponding (i.e. incongruent) target stimulus. This idea is similar to the assumption of source confusion yielding positive priming when stimuli are presented in close succession (Huber, 2008).

However, since we cannot prove whether or not a common mechanism is involved in the generation of inverse priming with both types of masks we next aimed to study the locus of inverse priming with irrelevant masks. An irrelevant mask is characterized by the complete absence of task-relevant features. However, similarity among the mask and the masked stimulus improves masking (Breitmeyer, 1984; Kinsbourne, \& Warrington, 1972; Hellige, Walsh, Lawrence, \& Prasse, 1979) which is probably one important reason for choosing relevant masks in many early studies on inverse priming. Therefore, we intended to create an irrelevant mask which strongly reduces prime visibility. To do so, metacontrast masking is not a suitable technique because the characteristic feature of a metacontrast mask is that the mask contains an inner cut-out of the same or at least a similar shape as the masked stimuli. Therefore, we explored pattern masking, a different backward masking technique (Breitmeyer, \& Ögmen, 2006). We also had to substitute the prime stimuli because masking of the plane shape stimuli should be more difficult than masking thin lines. Therefore, we chose the broadly employed double arrow stimuli (e.g. Eimer, \& Schlaghecken, 1998).

\subsection{Project II: Crucial Features of relevant masks}

The major aim of Project II was to isolate the crucial feature of a relevant mask. Numerous studies on inverse priming combined double arrow prime and target stimuli with random line masks (RLMs; Aron, et al., 2003; Boy, Evans, et al., 2010; Boy, Husain, et al., 2010; Eimer, \& Schlaghecken, 2002; Jaśkowski, 2007; 2008; Maylor, Birak, \& Schlaghecken, 2011; Schlaghecken, \& Eimer, 2002; 2004; 2006; Schlaghecken, et al., 2009; Sumner, Tsai, Yu, \& Nachev, 2006; Sumner, et al., 2007; Sumner, \& Brandwood, 2008; Wilson, et al., 2010). Such RLMs are constructed by overlaying multiple lines of different length and often also of different orientations. When including oblique lines the masking stimulus could also randomly entail target-like elements in a very abstract fashion (Lleras, \& Enns, 2004). Intersecting lines might form arrow heads within the structure of the mask. Such a mask could be considered a relevant mask when double arrows are used as primes and targets because all those arrow-head elements within the mask pointing to the opposite direction as the prime might be especially salient as a result of Object Updating. Then, due to this special visual impression larger inverse priming effects might result as compared to inverse priming with a 
completely irrelevant mask. Prior studies came to different conclusions about whether the visual system is capable of extracting the target features from the complex visual pattern of a RLM. Eimer and Schlaghecken (2008) found inverse priming effects to be about twice as large for RLMs containing oblique lines relative to an irrelevant mask which consisted of only horizontally and vertically arranged lines. Sumner (2008) on the other hand found equal effects for an RLM comprising target orientations and one sparing them out.

Thus, we systematically varied the similarity of the mask structure to the prime and target features in order to find out at which point the perceptual interaction of prime and mask produces larger effects compared to a completely irrelevant mask which was called the orthogonal mask (Krüger, \& Mattler, in prep., see Appendix II). The four masks employed are depicted along with the other stimuli in Figure 2. For each further mask tested, the diagonal mask, the arrow pattern mask and the superposition mask, we compared inverse priming effects to this reference mask.

We unambiguously showed that inverse priming effects were only more pronounced for the superposition mask which is built by directly superimposing the two stimulus alternatives. In contrast, inverse priming effects were found to be indistinguishable among the three types of RLMs tested. Even the arrow pattern mask which directly entailed the features forming primes and target did not produce larger effects (cf. Figure 2 from Krüger, \& Mattler, in prep., Appendix II). In addition, we observed an analogous categorical distinction in the continuum

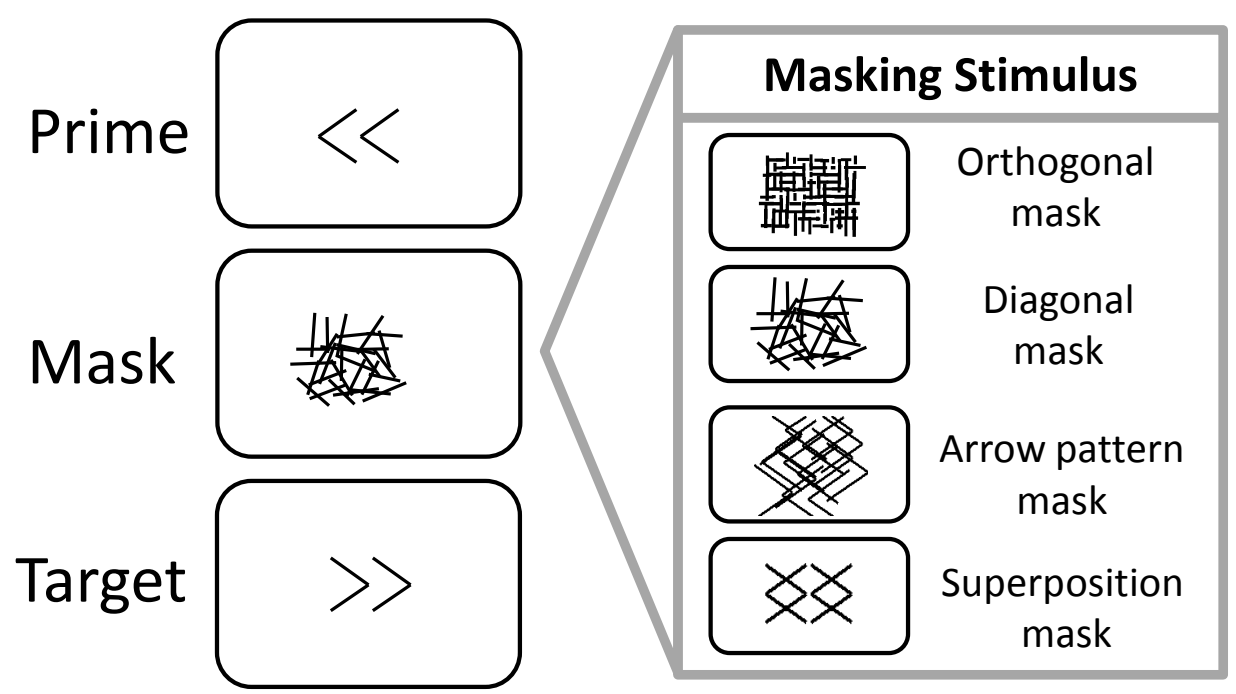

Figure 2. Illustration of the stimuli used in Project II. Primes and targets were always identical double arrows. The structure of the masking stimulus varied as a between-subject factor. 
of masking stimuli with respect to the relationship of prime visibility and the size of priming effects. Prime visibility was assessed in an independent direct prime recognition task which yielded a measure of how well subjects could perceive the masked prime. We observed a relatively strong positive linear relationship of prime visibility and priming effects for all masks $(r>.49)$ except for the superposition mask $(r=-.02$; cf. Figure 3 from Krüger, \& Mattler, in prep., Appendix II). However, while the vast majority of subjects performed rather poor on the prime recognition task, few subjects possessed a remarkable capacity to recognize the primes. Cluster-analytical results suggest that only four out of 54 subjects belonged to this category. These four subjects performing exceptionally well in the direct task were part of the orthogonal and diagonal mask condition. Potentially, data from these subjects could largely account for the observed linear relationship of prime visibility and priming effects (cf. Figure 3, Krüger, \& Mattler, in prep., Appendix II). Nevertheless, even when excluding these subjects, we still found a positive correlation of priming effects and prime visibility for the three RLMs ( $\mathrm{r}>.43)$.

Thus, the superposition mask seems to be distinct from the other masks with respect to two independent aspects. First, it is the only mask which seems to boost inverse priming due to a perceptual interaction of prime and mask. Second, inverse priming effects with the superposition mask turned out to be independent of prime visibility while a positive relation was found for the other masks implying larger inverse priming effects within those subjects being less able to recognize the primes. Such a differential impact of prime visibility for the magnitude of inverse priming effects with relevant as opposed to irrelevant masks has been discussed before (Klapp, 2005). Consequently, the visual system seems to be incapable of extracting the task-relevant features from the RLMs employed. In contrast, sequential presentation of the superposition mask might generate the perception of two successive onsets: first the prime, then (with mask onset) the opposite stimulus (Verleger et al., 2004). However, others also demonstrated that RLMs similar to our arrow-pattern mask but of a less complex structure (i.e. built of less arrow elements) also lead to an increase of inverse priming effects after extensive training (Przekoracka-Krawczyk, \& Jaśkowski, 2007). This finding suggests that the extractability of target features from the mask can be improved by perceptual learning. Potentially, with more training an additional mask-induced effect would have also been possible with our relatively complex arrow-pattern mask.

Getting back to the model outlined in the introduction, we also replicated that inverse priming effects with response-compatible arrow stimuli were about twice as large with relevant as 
opposed to irrelevant masks. In addition, apart from the relation of prime visibility and priming effects the time-course also differed for the two types of masks suggesting that two independent mechanisms might be involved in the generation of inverse priming with the two types of masks.

Having now established the distinguishing feature of relevant and irrelevant masks, we next aimed to examine the origin of inverse priming with such irrelevant masks. In Project I, we found evidence for the view that the inverse priming effect with relevant masks is completely generated at perceptual levels of processing. In Project III we focused on irrelevant masks and studied response-compatible and non-compatible stimuli.

\subsection{Project III: Stimulus-response compatibility and inverse priming}

Project III was initially stimulated by the question where inverse priming with irrelevant masks originates. To this end, we implemented an experimental paradigm which had been successfully used before to study the locus of inverse priming with relevant masks (Krüger, \& Mattler, in prep., see Appendix III). Compared to the PRP-paradigm applied in Project I, this paradigm is based on fewer assumptions. In particular, the locus of priming effects is not determined relative to a hypothetical processing bottleneck which is assumed to be located at central levels (Pashler, \& Johnson, 1998; for a critical discussion of how PRP-results should be interpreted see also: Klapötke, Krüger, \& Mattler, 2011). The basic idea of the paradigm used here is to randomly intermix two sets of target stimuli. One set is identical to the prime set, the other one is perceptually dissimilar but requires the same responses. For example, Eimer (1999) used double arrows as prime stimuli which were also presented on half of all trials as target stimuli. In addition, on the remaining trials a lateral "+"-sign appeared which required the same responses as the centrally presented double arrow target. If inverse priming is indeed a motor phenomenon relying on the inhibition of the primed response, the effect should be equally pronounced with both types of target stimuli. If, for instance, a leftward pointing double arrow activates the left response which is then inhibited by the processing of the mask, performance with both types of congruent targets, a leftward pointing double arrow or a "+"-sign on the left-hand side, should suffer compared to a target assigned to a right-hand response. On the other hand, perceptual accounts of inverse priming would predict inverse priming only for targets that are identical or at least similar to the primes because the hampered perceptual analysis on congruent trials is due to repeated presentation of the same stimulus (Huber, 2008; Sohrabi, \& West, 2009). 
Previous experiments with relevant masks demonstrated a complete transfer of inverse effects to dissimilar target stimuli (Eimer, 1999; Klapp, \& Hinkley, 2002). In Experiment 1, we replicated Eimer's (1999) experiment using irrelevant masks. Thus, primes were double arrows which were followed on half of the trials by double arrow targets and on the remaining trials by a lateral "+"-sign. In contrast to the results obtained for relevant masks (Eimer, 1999; Klapp, \& Hinkley, 2002), we only found a partial transfer of priming effects to lateral targets indicating the involvement of both, perceptual and post-perceptual parts, in the generation of inverse priming with irrelevant masks (cf. Figure 2 and Figure 3 from Krüger, \& Mattler, in prep., Appendix III). However, in contrast to the studies with relevant masks (Eimer, 1999; Klapp, \& Hinkley, 2002), we also observed faster responses for lateral target stimuli compared to central targets. Motor inhibition, however, is conceptualized as a process which critically depends on the response time (Schlaghecken, \& Eimer, 2000). Electrophysiological evidence demonstrated a biphasic pattern of priming effects (Eimer, \& Schlaghecken, 1998; 2003; Praamstra, \& Seiss, 2005). Relative to the prime onset, motor activity as reflected in LRPs was initially biased towards the primed response and then shortly after mask presentation reversed such that the unprimed response gained an activation advantage. Thus, if responses are too fast, activation might be still governed by the prime and inhibition did not fully exhibit its influence. To prove this hypothesis, we assessed priming effects separately for different absolute response times. We, therefore, divided the distribution into 19 percentiles and found that inverse priming was restricted to slowest responses $\left(15^{\text {th }}, 20^{\text {th }}, \ldots, 95^{\text {th }}\right.$ percentile) but was absent for the fastest responses $\left(5^{\text {th }}, 10^{\text {th }}\right.$ percentile). This results are confirmed by a recent study by Schlaghecken and colleagues who reported initial positive priming effects for fast responses (relative to prime offset) and inverse effects for slower responses (Schlaghecken, et al., in press). Hence, the overall bias of the RT distribution for lateral targets towards faster responses compared to responses to central targets, might have yielded a smaller inverse priming effect which at first glance reflects only a partial transfer of effects to lateral targets. To test this, we split the distributions for both types of targets and compared priming effects for the $20^{\text {th }}, 40^{\text {th }}, 60^{\text {th }}$ and $80^{\text {th }}$ percentile of the distribution (cf. Schlaghecken, \& Eimer, 2000). We indeed found that effects were only smaller for lateral targets for the fastest responses $\left(20^{\text {th }}\right.$ and $40^{\text {th }}$ percentile) but indistinguishable for slowest responses $\left(60^{\text {th }}\right.$ and $80^{\text {th }}$ percentile). Thus, when controlling for overall response speed, we observed a full transfer of priming effects to lateral targets suggesting that the mechanism mediating inverse priming is entirely located at post-perceptual levels of processing. These results exactly replicate results for arrow stimuli masked by a relevant mask in a study which 
also observed faster responses for lateral targets and a partial transfer of effects to lateral targets which disappeared when considering only slowest responses (Schlaghecken, \& Eimer, 2000). In contrast to previous results with relevant masks and non-response compatible stimuli (Mattler, 2006), the visual similarity of primes and targets seemed to be completely irrelevant for the behavioral outcome.

So far, Experiment 1 provided evidence for a post-perceptual locus of inverse priming effects with irrelevant masks and double arrow prime stimuli. This is suggested by a complete transfer of priming effects to perceptually dissimilar targets which, however, require the same responses as the prime stimuli when the different absolute response speed for central and such lateral targets is also taken into account. In Project III, eight further experiments were conducted in order to test the generality of this finding for other prime stimuli. To our surprise, we completely failed to find evidence for inverse priming even for central targets (which are identical to the prime stimuli) except when employing bar pair stimuli (Experiment 4b) or single arrow stimuli (Experiment 8). All stimuli are depicted in Figure 3.

\begin{tabular}{|c|c|c|c|}
\hline & $\begin{array}{c}\text { Primes / } \\
\text { Central Targets }\end{array}$ & Masks & $\begin{array}{l}\text { Inverse } \\
\text { Priming } \\
\end{array}$ \\
\hline Exp. 1 & 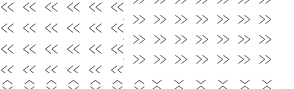 & 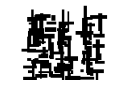 & Yes \\
\hline Exp. 2 & 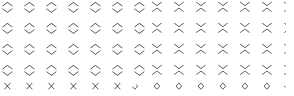 & 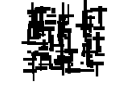 & No \\
\hline Exp. 3+9 & & 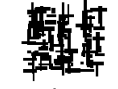 & No \\
\hline Exp. $4 a+4 b$ & & 似 & Yes (Exp. 4b) \\
\hline Exp. 5 & & 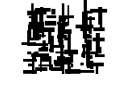 & No \\
\hline Exp. 6 & & 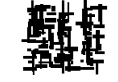 & No \\
\hline Exp. 7 & & 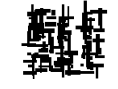 & No \\
\hline Exp. 8 & & 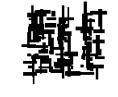 & Yes \\
\hline
\end{tabular}

Figure 3. Stimuli used in Experiment 1-9 of Project III. All experiments employed irrelevant masks while the structure of prime and central target stimuli changed across the experiments. Apart from arrow stimuli (Exp. 1, 8), we employed other stimuli with response compatible elements (Experiment 5, 6, 7) and response noncompatible stimuli (Exp. 2, 3, 4a, 4b, 9). Apart from Experiment 4b and 9, on half of all trials, a lateral target was presented which is not depicted. Even for central targets which were always identical to prime stimuli, inverse priming was only found for double arrows (Exp. 1) and single arrows (Exp.8). In addition, inverse priming was also found with bar pair stimuli but only when lateral targets were omitted (Experiment $4 \mathrm{~b}$ ) but not when they were included (Experiment 4a). 
Using the same experimental paradigm, we failed to obtain inverse priming with various different non-arrow stimuli like objects that are symmetrical relative to the spatial alignment of responses (Experiment 2, 3 and 9) and more complex stimuli which possess responsecompatible elements (Experiment 5,6 and 7) even when restricting the analysis to those subjects being unable to perceive the primes. Inverse priming was even absent when subjects were practicing the SR-mappings for five sessions (Experiment 9) which seems to play a crucial role in inverse priming (Boy, \& Sumner, 2010). We also prolonged SOAs to up to 300 $\mathrm{ms}$ to test whether the onset of inverse priming is delayed but still failed to observe the effect (Experiment 9). Furthermore, additional analyses suggested that differences with respect to overall response speed or prime visibility between arrow and such non-arrow stimuli cannot account for the absence of the effect.

Unequivocal evidence for inverse priming was only obtained with double arrow stimuli (Experiment 1) and also for single arrow stimuli when restricting the analysis to subjects showing chance-level performance on the direct prime recognition task (Experiment 8). In addition, horizontal and vertical bar pairs masked by RLMs of oblique lines (Experiment 4b) which are supposed to be irrelevant at first glance also led to inverse priming which is consistent with previous findings (Boy, et al., 2008; Boy, \& Sumner, 2010; Sumner, 2008). However, effects were only found when lateral targets were omitted (Experiment 4b) but not when they were part of the experiment (Experiment $4 \mathrm{a}$ ). As a further distinguishing feature, inverse priming effects were only detectable in the second session while effects were present right from the beginning with double arrows. Such a delayed onset of priming effects has been repeatedly shown when relevant masks were used (Klapp, \& Hinkley, 2002; Przekoracka-Krawczyk, \& Jaśkowski, 2007) which might suggest that the oblique line masks are in fact relevant masks for the bar pair stimuli, too. Notably, the elements forming the bar pairs are physically most similar to the elements building the masks when compared to the other stimuli tested (see Figure 3). Increased relative salience of new mask features (due to Object Updating) might have therefore introduced a bias facilitating the processing of incongruent targets. However, integration of vertically and horizontally oriented lines into the random line masks, which is expected to further facilitate perceptual prime-mask interactions, does not enlarge inverse priming effects of the bar pair stimuli (Sumner, 2008).

If one accepts that the bar pair effect is induced by the special design of the mask, inverse priming seems to be limited to arrow stimuli. Demonstrations of inverse priming with nonarrow stimuli are rather rare when a mask-induced effect is excluded by the use of an 
irrelevant mask. Three examples can be found in the literature on inverse priming: Jaśkowski \& Ślósarek (2007) used double rectangular brackets ([[ vs. ]]), Verleger and others (2005) used two parallel horizontal lines which are intersected by one vertical line on either the lefthand or right-hand side, and Schlaghecken and colleagues (2007) used Landolt rings with the gap either on the left-hand or right-hand side. Like arrows, all these stimuli have a spatial layout that is compatible to the response set. The existence of inverse priming with these stimuli suggest that the spatial compatibility is their common decisive feature and that arrows are not special simply because they are over-learned symbols associated with the concepts of "left" and "right". This view is further strengthened by the demonstration of unaffected inverse priming effects with double arrow stimuli when response assignments are reversed (<< requires right response and $\gg>$ left response; Jaśkowski, \& Ślósarek, 2007; O’Connor, \& Neill, 2011). In contrast to these stimuli which produced inverse priming, the responsecompatible stimuli tested in Experiment 5 to 7 might not be treated as entities by the visual system. Instead of holistic stimulus processing, spatial attention might shift to the taskrelevant feature as also suggested by post-hoc interviews with the participants. In opposition, response-compatible objects might bypass controlled response selection and can directly affect motor processes (Kornblum, et al., 1990; Eimer, 1993, 1995; Eimer, Hommel, \& Prinz, 1995) which could be critical for inverse priming with irrelevant masks. After the initial automatic response activation induced by the prime, motor inhibition might generate inverse priming. If inverse priming effects with irrelevant masks are indeed a motor phenomenon, the effects should not be found in a non-motor cue-priming paradigm. This was tested in Project IV.

\subsection{Project IV: Inverse non-motor priming}

Non-motor priming is a straightforward extension of the traditional motor priming paradigm which was introduced by Mattler (2003). Instead of three stimuli, the stimulus sequence comprises an additional target stimulus in inverse non-motor priming. The other stimuli are identical in both paradigms: primes are followed by a masking stimulus which is followed by third stimulus (the target in motor priming). Instead of requiring a specific motor response, this third stimulus serves as a cue announcing which one of two alternative tasks has to be performed with respect to the upcoming target stimulus. Just like in motor priming, primes and cues can either announce the same task (congruent condition) or different tasks 
(incongruent condition). The inverse non-motor priming effect consists in better performance in the incongruent condition as opposed to the congruent condition (Mattler, 2006; 2007).

Due to the astonishing analogies between motor and non-motor priming with respect to the role of prime visibility and the time course of the effects (Mattler, 2003; 2007), it seems reasonable to assume a common underlying mechanism producing both effects. Therefore, the application of inverse non-motor priming can provide important insights in the study of this common mechanism. Because inverse non-motor priming cannot originate from the motor system, the existence of the effect with irrelevant masks would conflict with the idea of a crucial involvement of a direct translation route from perceptual analysis to motor output in inverse priming with irrelevant masks. To test this, Project IV comprised three experiments applying a new variant of the non-motor priming paradigm developed by Mattler $(2006 ; 2007$; Krüger, \& Mattler, in prep., see Appendix IV). In Experiment 1, the new paradigm was first introduced using a relevant mask while other aspects were very similar to the experiments carried out by Mattler (2007, Exp. 2 and 3). However, using a relevant mask leaves open the possibility that effects are due to perceptual interactions of primes and masks rendering prime-incongruent parts of the mask more salient which facilitates preparation of the primeincongruent task. Therefore, we conducted Experiment 2 and 3 which studied inverse nonmotor priming with irrelevant masks. We used the orthogonal mask also employed in Project II and III and combined it with either double arrow primes and cues (Experiment 2) or the arrow pattern stimuli (Experiment 3). The stimuli are depicted in Figure 4.

\begin{tabular}{|c|c|c|c|c|}
\hline & Prime & Mask & Cue & Target \\
\hline \multicolumn{5}{|l|}{ Exp. 1} \\
\hline \multirow{3}{*}{ Exp. 2} & 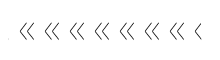 & \multirow{3}{*}{ 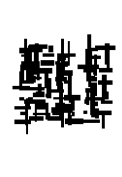 } & 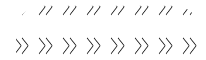 & \\
\hline & 《《《《《《《《《 & & 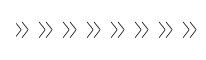 & \\
\hline & 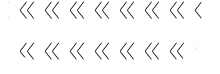 & & 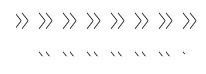 & \\
\hline \multirow{3}{*}{ Exp. 3} & $\hat{v} \hat{v} \hat{v} \hat{v} \hat{v} \hat{v} \hat{v} \hat{v}$ & \multirow{3}{*}{ 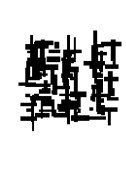 } & $\therefore x x x x x x x$ & \\
\hline & $\hat{v} \hat{v} \hat{v} \hat{v} \hat{v} \hat{v} \hat{v} \hat{v}$ & & $x x x x x x x x$ & \\
\hline & $\begin{array}{l}\hat{v} \hat{v} \hat{v} \hat{v} \hat{v} \hat{v} \hat{v} \hat{v} \\
\hat{v} \hat{v} \hat{v} \hat{v} \hat{v} \hat{v} \hat{v}\end{array}$ & & $\begin{array}{l}X X X X X X X X \\
\therefore X X X X X X X X\end{array}$ & \\
\hline
\end{tabular}

Figure 4. Stimuli used in Project IV. Experiment 1 used a relevant mask, Experiment 2 and 3 irrelevant masks. Target stimuli were the same throughout the entire series of experiments and consisted of two superimposed photographs. One (out of 16) instance is depicted here. Note also that primes and cues are non-compatible to the tasks since there is no obvious common dimension between the two alternative tasks and the stimuli (see General Discussion). 
Subjects were required to perform one out of two alternative tasks on a complex target stimulus which was composed of two overlaid photographs, one depicting a human face, the other one a visual scene. The preceding cue indicated on which aspect of the target stimulus subjects had to focus. One cue required the subject to attend to the face and report whether it is a female (left response) or male face (right response). The alternative cue required the subject to attend to the scene reporting whether the depicted setting is located indoor (left response) or outdoor (right response). Previous to cue onset, a prime followed by a mask were shown. Apart from prime-cue congruency, we also manipulated the mask-cue SOA which proved to be a crucial determinant of the size of inverse non-motor priming effects (Mattler, 2007). All in all, subjects performed four sessions, the first one serving to practice the task, the last one was a prime recognition session.

In Experiment 1, we replicated inverse non-motor priming with relevant masks using the new paradigm. Corroborating previous findings (Mattler, 2007), inverse priming effects were independent of the individual ability to recognize the prime stimuli. Evidence for inverse nonmotor priming with irrelevant masks in Experiment 2 and 3 was more ambiguous. Even though we included data from two one-hour sessions of 24 subjects in each experiment, we almost completely failed to observe a stable inverse non-motor priming effect across the entire sample (an inverse effect on error rates became manifest in Experiment 3). However, we further found positive correlations across subjects between individual priming effects and prime visibility data, especially in Experiment 2, i.e. inverse effects were more pronounced among those subjects being unable to consciously perceive the primes. This result led us to divide the entire sample according to prime visibility. A subgroup of 8 participants per experiment, showed prime recognition performance indistinguishable from chance-level. Among these subjects we observed a stable inverse priming effect across the entire range of mask-cue SOAs tested in Experiment 2. In Experiment 3, the size of inverse priming effects depended on the mask-cue SOA and we only found an inverse effect for the 150-ms-SOA.

To sum up, in contrast to relevant masks, inverse non-motor priming with irrelevant masks seems to be rather instable and was only observed among those subjects showing subliminal prime perception. The modulation of inverse priming effects with irrelevant masks by masking efficiency is in line with analogous findings from Project II (all but the superposition mask) and Project III (Experiment 1; see Krüger, \& Mattler, in prep., Appendix III). The existence of inverse non-motor priming with irrelevant masks casts doubts on the idea that a direct link to the motor system is essential for inverse priming to occur. On the other hand, the 
small effect might result from a different mechanism as inverse motor priming. This issue will be further addressed in the General Discussion.

Project I to IV provided evidence for discernible mechanisms involved in the generation of inverse priming with response-compatible and non-compatible stimuli. Disregarding the small but stable non-motor priming effect in Project IV, response non-compatible stimuli seem to require the use of a relevant mask to achieve inverse priming (Project I and III, similar results were also reported by Jaśkowski, \& Ślósarek, 2007; Kiesel, et al., 2008). Under these conditions, the effect seems to arise completely from perceptual levels of processing (Project I, Mattler, 2006) supposedly because perceptual processing of incongruent targets is facilitated due to enhanced processing of prime-incongruent mask features. On the other hand, inverse priming with response-compatible stimuli like arrows was demonstrated for both types of masks (Project II and III, e.g. Eimer, \& Schlaghecken, 1998; Schlaghecken, \& Eimer, 2006; Schlaghecken, et al., 2007). In opposition to the mask-induced effect with relevant masks, this effect seems to be completely due to motor processes irrespective of whether a relevant (Eimer, 1999; Klapp, \& Haas, 2005; Klapp, \& Hinkley, 2002) or an irrelevant mask is used (Project III, Boy, Evans, et al., 2010; Boy, Husain, et al., 2010; Klapp, 2005; Schlaghecken, et al., 2009; Sumner, et al., 2007). Thus, the perceptual effect which generates the effect with relevant masks when non-compatible stimuli are used is not detectable anymore with compatible stimuli. Such compatible stimuli might directly activate their corresponding response (Georgopoulos, et al., 1989; Kornblum, et al., 1990; Eimer, 1993, 1995; Eimer, et al., 1995; Procter, et al., 1995) and the inhibition-based reversal due to the mask might only be reinforced when a relevant mask is used. To test whether inverse priming with response-compatible stimuli truly originates from such a mechanism located at motor levels of processing we applied functional magnetic resonance imaging in order to reveal the neural substrates underlying this effect.

\subsection{Project V: Imaging control operations in inverse priming}

In Project V, we applied functional magnetic resonance imaging (fMRI) to study the anatomical location of the mechanism underlying inverse priming with response-compatible stimuli for both types of masks (Krüger, Klapötke, Bode, \& Mattler, submitted, see Appendix V). With such response-compatible stimuli, the results obtained so far suggest that primes can directly activate motor preparation processes which are subject to motor inhibition. The regulation of these motor preparation processes involves cognitive control operations 
(Ridderinkoff, Wildenberg, Segalowitz, \& Carter, 2004). Consequently, these control processes would also be expected to be located in the motor system. In contrast, it would be also possible that control operations in inverse priming intervene with processing on ealier levels such that activation of competing perceptual representations is altered by cognitive control. We applied fMRI to investigate whether control operations in inverse priming with response-compatible stimuli are indeed located within the motor system or whether they are also detectable at perceptual levels of processing.

Imaging inverse priming requires a concrete model of the processes underlying the effect from which predictions can be derived concerning the expected neural effects. Based on the evidence gathered so far and in line with current motor accounts on inverse priming (Boy, Husain \& et al., 2010; Jaśkowski, \& Verleger, 2007; Lleras, \& Enns, 2006), we hypothesized that inverse priming reflects a series of control processes which become apparent in dynamic changes of motor preparation processes. These motor activations can be illustrated by electrophysiological recordings over the motor cortices. Then, the LRP can be calculated which is suitable to infer the underlying motor preparation processes (Colebatch, 2007). In inverse priming, a three-phasic pattern of motor activation processes has been repeatedly demonstrated (Eimer, \& Schlaghecken, 1998, 2003; Praamstra, \& Seiss, 2005). An initial activation phase of the response associated with the prime is followed by a reversal probably due to mask presentation. While incongruent targets simply continue this reversed tendency until a response threshold is reached, congruent targets induce a further reversal and redirect activation accumulation in the initial prime-induced direction (see Figure 5, right column). Consequently, on congruent trials a second, independent compensation of the misleading mask-induced reversal is required which is unnecessary on incongruent trials. The abortion of such premature response tendencies is supposed to be accomplished by a central cognitive control instance moderating response competition (Ridderinkoff, et al., 2004). The aim of the present MRI study was to localize the anatomical source of this control instance in inverse priming. We expected to find either evidence that cognitive control affects processing at perceptual or post-perceptual levels.

Previous physiological evidence suggests that the control instance is directly involved in the regulation of motor activation (Boy, Evans, et al., 2010; Boy, \& Husain, et al., 2010; Sumner, et al., 2007). Then, the neural correlate of cognitive control in inverse priming would be expected to be part of the motor system. However, it is also plausible that an early perceptual mechanism controls activation of perceptual representation of the two stimulus alternatives 
(instead of controlling response preparation processes). From this perspective, motor processes as reflected in the LRP are only secondary consequences of the early competition. Supposedly, then the neural site of control would also be located in the visual system.

To obtain an optimal measure of control processes in inverse priming we systematically varied regulatory demands by implementing four different experimental conditions for each mask type. These conditions resulted from factorial combination of a short (33 ms) and long mask-target SOA condition $(150 \mathrm{~ms})$ with congruent and incongruent primes and targets. At short SOAs positive priming is expected while effects should reverse at long SOA (Boy, \& Sumner, 2010; Mattler, 2007; Schlaghecken, \& Eimer, 2000). As a neural measure of control demands we contrasted parameter estimates (of the general linear model fitted to the MRIdata) for the long and short SOA in the congruent condition. On congruent trials, increasing SOA enlarges regulatory demands because the misleading tendency towards the wrong response is lasting longer. Thus, conflict between ongoing task processing and target processing grows and consequently correction requires more cognitive effort. However, increasing SOA does not only manipulate control demands but is of course accompanied by corresponding physical differences in the temporal structure of the stimulus sequence which might also affect neural activity. To control for such confounded control-unrelated SOA effects, we considered the main effect of SOA on incongruent trials which involves the same perceptual effect whereas control operations are supposed to be unaffected by SOA changes on incongruent trials. This is because on incongruent trials only an initial mask-induced reversal occurs and target presentation simply confirms the reversed tendency irrespective of when the target is presented. Target presentation does not necessitate any change in response preparation processes and consequently, additional compensatory control operations are not required at all. This model is illustrated in Figure 5.

Three predictions can be derived from this model of the role of cognitive control in inverse priming. 1) Because changing SOA on incongruent trials does only reflect physical changes in the stimulus sequence a corresponding neural affect should be restricted to the visual system. 2) Because the same visual effect should also occur on congruent trials, the activity in the same visual areas should be susceptible to SOA variation on congruent trials too. 3) While SOA effects on incongruent trials should be limited to the visual system, further SOA effects are expected to be found in the congruent condition reflecting varying control demands. Assessing the anatomical locus of these congruency-specific SOA effects allows us to infer the origin of control operations in inverse priming. 
SOA $33 \mathrm{~ms}$
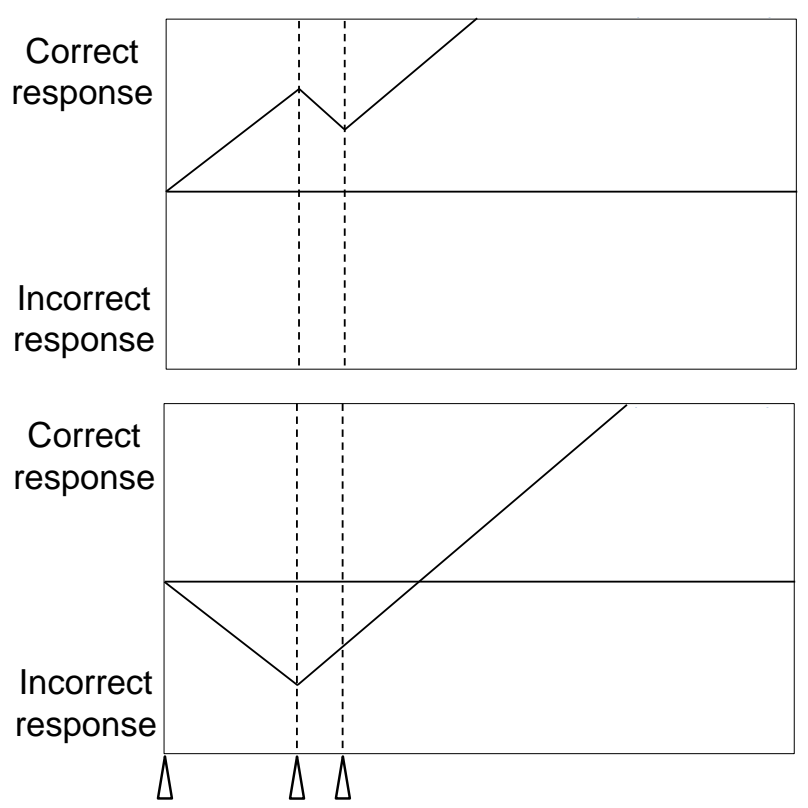

ह ह ह है के
SOA $150 \mathrm{~ms}$
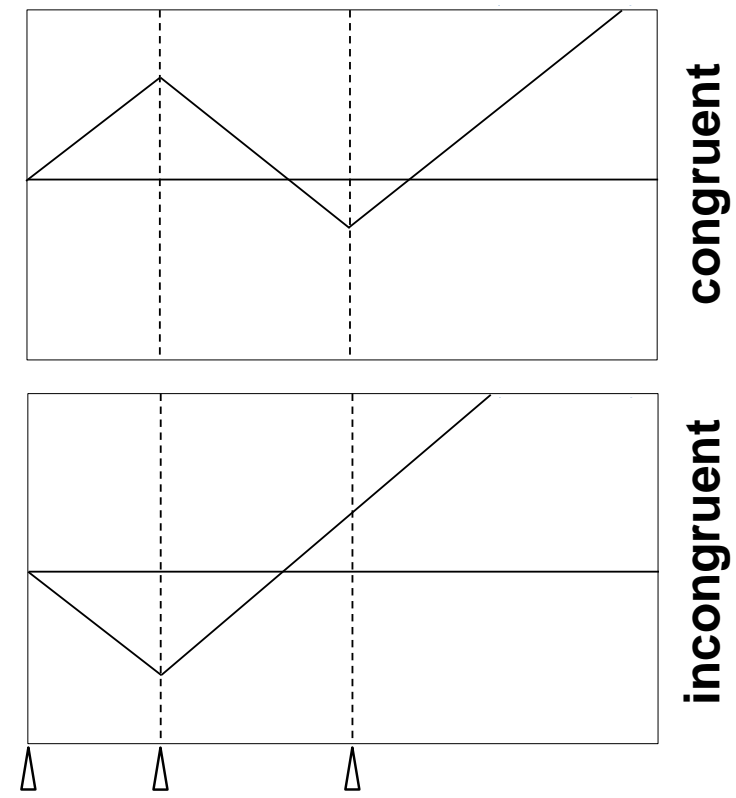

Figure 5. Response activation model for inverse priming. The model is based on electrophysiological research demonstrating the time course of LRPs in inverse priming (Eimer, \& Schlaghecken, 1998; 2003; Seiss, \& Praamstra, 2005). The figure illustrates the time course of the activation difference of left- and right-hand responses for congruent (upper row) and incongruent trials (lower row) as well as short (left column) and long SOA (right column). Three consecutive phases can be distinguished: first, the prime activates the associated response which is the correct one on congruent trials and otherwise the incorrect one. Second, the presentation of the mask reverses prime-related activation and drives it towards the unprimed response alternative until target presentation. Third, targets contribute to differential response preparation. Congruent targets induce a further reversal while incongruent targets simply lead to a continuation of mask-induced activation. The model assumes that reversals of activation processes are triggered by control operations. The regulatory demands related to these control operations depend on mask-target SOA on congruent trials. With increasing mask-target SOA, the maskinduced misleading bias also increases and compensation by target processing requires more and more resources such that regulatory demands are larger. Note that, regulatory demands are independent of SOA on incongruent trials because only the mask-induced reversal occurs. Therefore, neural activation which increases with SOA on congruent trials but not on incongruent trials reflects the increase of regulatory demands and associated control processes in inverse priming.

The MRI results obtained nicely fitted with these predictions. SOA effects on both, congruent and incongruent trials, were observed for both types of masks only in higher areas of the visual system at the transition from occipital to temporal lobe (V5/MT+, Malikovich, et al., 2007, cf. Figure 4 from Krüger, et al., submitted; Appendix V). These areas are involved in motion perception (Wilms, et al., 2005) and were previously related to the processing of 
apparent motion (Goebel, Khorram-Sefat, Muckli, Hacker, \& Singer, 1998). Probably, the employed stimulus sequence (cf. Figure 1 from Krüger, et al., submitted; Appendix V) also induced motion signals in our participants which are supposed to be more pronounced at long SOA. The importance of the temporal delay between the stimuli for the perception of apparent motion has been demonstrated before (Shepard, \& Zare, 1983).

In addition to these SOA effects common to both Congruency conditions, several brain regions showed SOA effects on congruent but importantly not on incongruent trials even at a rather liberal threshold (cf. Figure 5 and Table 3 from Krüger, et al., submitted, Appendix V). Such control specific neural effects were most pronounced in the supplementary motor area (SMA) and common to both types of masks. In large parts of the SMA, the individual increase in activity from short to long SOA in the congruent condition was correlated to the priming effects on RTs (for both masks, see Figure 6 from Krüger, et al., submitted, Appendix V). Moreover, several areas of the parietal and frontal cortex were specifically involved in control operations with relevant masks but not with irrelevant masks. These areas included the rostral cingulate zone (Picard, \& Strick, 1996), bilateral insula, the left postcentral gyrus and left supramarginal gyrus potentially reflecting a control network specifically involved with relevant masks.

Thus, application of fMRI to the inverse priming paradigm provided further support for a motor locus when response-compatible arrow stimuli are used. The foci were located in the SMA which has previously been found to be crucial for inverse priming (Boy, Evans, et al., 2010; Boy, \& Husain, et al., 2010; Sumner, et al., 2007). The moderating function of the SMA with respect to conflicting response tendencies has also been demonstrated in the Eriksen Flanker Task (Hazeltine, 1990). Moreover, suppression of inappropriate response tendencies (Swick, et al., 2011), sudden adaptation of instantiated motor plans (Matsuzaka, \& Tanji, 1996), response inhibition in reaction to sudden task changes (Chen, Scangos, \& Stuphorn, 2010) or in general the instantiation and control of relevant movements (Nachev, et al., 2008) have also been related to the SMA. Thus, the SMA seems to be the central motor control component regulating response preparation processes in inverse priming irrespective of which type of mask is used. With relevant masks, further components come into play augmenting the effect. 


\section{General Discussion}

Five projects examined the role of stimulus design in inverse priming which was suspected to be the crucial determinant of the large variability of inverse priming effects reported in the literature. Moreover, the idea was put forward that the same observable phenomenon, the inverse priming effect, is produced differently according to the stimulus characteristics. A taxonomic classification of inverse priming effects based on the response-compatibility of prime and target stimuli on the one hand and the structure of masking stimuli on the other hand has been proposed.

In the following, the mechanisms underlying inverse priming with the distinct types of stimuli will be discussed incorporating the most important findings of the five projects. First, inverse priming with response-compatible stimuli is addressed. Because we only employed arrow stimuli, it is discussed whether inverse priming with arrows is representative for the broader class of all response-compatible stimuli. Then, the mechanism underlying inverse priming with response non-compatible stimuli is considered. In particular, the divergent results from motor and non-motor priming concerning inverse priming with response non-compatible stimuli combined with irrelevant masks are discussed. Finally, implications of our results for two important issues in the study of inverse priming are addressed. The data are reanalyzed to reveal possible relations between priming effects and prime visibility. Furthermore, it is discussed whether a common mechanism is involved in inverse priming with relevant and irrelevant masks or whether the effect results from two completely separate mechanisms with both types of masks.

\subsection{Inverse priming with response-compatible stimuli}

Almost all empirical accounts of inverse priming used response-compatible double arrow stimuli. Comparatively large effects result when such stimuli are combined with relevant masks (e.g. Eimer, \& Schlaghecken, 1998; Project II and V). These effects seem to arise completely at post-perceptual levels of processing as suggested by our MRI findings (Project V) and by further psychophysical studies demonstrating the complete transfer of the effect to perceptually dissimilar target stimuli (Eimer, 1999; Klapp, \& Hinkley, 2002; Schlaghecken, \& Eimer, 2002). A post-perceptual locus is also suggested by a recent study demonstrating a complete reversal of priming effects following an unexpected reversal of SR-mappings (Boy, $\&$ Sumner, 2010) and by the demonstration of free-choice priming effects with relevant masks 
(Klapp, \& Hinkley, 2002; O’Connor, \& Neill, 2011; Schlaghecken, \& Eimer, 2004). When irrelevant masks are used, such response-compatible stimuli consistently produced smaller effects (Schlaghecken, \& Eimer, 2006; Project II and V). Nonetheless, these inverse priming effects are still generated on a post-perceptual level as revealed by the complete transfer to perceptually dissimilar targets (Project III) and the restriction of control processes to motor areas (Project V; Boy, Evans, et al., 2010; Boy, Husain, et al., 2010; Sumner, et al., 2007). Additionally, inverse priming effects on freely chosen responses have also been demonstrated with irrelevant masks (Schlaghecken, et al., 2009).

Taken together, effects with response-compatible stimuli seem to be always generated on post-perceptual processing levels. Response-compatible stimuli have been assumed to automatically elicit motor preparation processes (Kornblum, et al., 1990; Eimer, et al., 1995). Georgopoulos and colleagues (1989), for instance, recorded activity from motor cells tuned to a specific movement direction and computed the population vector as a weighted sum of the activity of the recorded neurons. Rhesus monkeys either had to move a handle towards or perpendicular to a visual stimulus. Even when required to perform the movement perpendicular to the stimulus an initial motor activation towards the stimulus was observed which was subsequently replaced by the correct response. This rapid motor activation consistent with the stimulus input might result from automatic SR-translation regardless of task requirements. Beyond this, Eimer $(1993,1995)$ reported a comparable stimulus-induced rapid motor activation in humans illustrated by LRPs. In a cuing paradigm, an arrow cue pointing either left- or rightward was followed by a target stimulus. Responses congruent to the cue were activated about $200 \mathrm{~ms}$ after cue onset although the cue was not predictive for the upcoming response (or even counter-predictive, i.e. announcing the wrong response on 75 $\%$ of all trials). In contrast, a non-spatial color cue did not elicit such motor effects (Eimer, 1995). These results were corroborated in a similar cuing paradigm demonstrating extended lateralized activity consistent with the orientation of an arrow precue irrespective of its predictive validity with respect to the target (Verleger, Vollmer, Waschkuhn, van der Lubbe, $\&$ Wascher, 2000). More recent results, demonstrated stronger activations of the respective primary motor areas when stimuli required a spatially corresponding response (compatible SR-mapping) as opposed to a response with the opposite effecter (incompatible SR-mapping). These magnetoencephalography results are consistent with an automatic contribution to response activations (Kato, Endo, Kizuka, \& Asami, 2006). Moreover, automatic activation of motor plans by visual stimuli is also suggested by inevitable saccades to suddenly appearing visual stimuli (the so-called "visual grasp reflex", Theeuwes, Kramer, Hahn, \& Irwin, 1998). 
Similarly, visual objects seem to automatically activate actions afforded by them. Graspable objects were more quickly identified as positioned upright or inverted when their graspable part (e.g. the handle of a mug) matched the spatial position of the response although the leftright orientation of the objects was completely irrelevant for the task (Tucker, \& Ellies, 1998; see also Derbyshire, Ellis, \& Tucker, 2006; Tucker, \& Ellis, 2004). Most strikingly, only recently, masked double arrow stimuli identical to those employed to study inverse priming were shown to induce activation in human premotor areas even though no movement had to be executed (D'Ostilio, \& Garraux, 2011). These masked double arrows were randomly intermixed with symmetrical prime stimuli (x). Immediately after prime offset, a mask and a pair of target stimuli presented left and right of the mask were shown simultaneously. Target stimuli were either also two double arrows or two circle stimuli. While double arrows required a corresponding response, circle stimuli signaled the subject to withhold the response. On these non-response trials, double arrow primes activated medial premotor cortex. The same area was also activated on response trials. In contrast, however, symmetrical primes failed to elicit such a cortical response on non-response trials. Primes were presented subliminally as indexed by chance-level performance on a direct prime recognition task. Thus, subliminal arrow stimuli seem to trigger in fact motor preparation at least when they are part of the current task set.

Such results were taken as evidence for a well-established account to explain SRcompatibility effects, the dimensional overlap model (Kornblum, et al., 1990). According to the model, two distinct processing routes translate stimulus input to motor output. One route is characterized by a controlled response-selection process which is essential when stimuli do not share any feature with responses. On the other hand, responses can be directly activated bypassing controlled response selection. Such automatic translation is involved when the stimulus and response set share some feature, i.e. both can be distinguished on a common underlying dimension. This situation is called dimensional overlap. For example, spatial correspondence in the simon task is assumed to automatically activate the spatially corresponding response while the correct response is determined on the second controlled translation route which operates in parallel (Leuthold, 2011). Various similar dual-route models have been proposed to account for SR-compatibility phenomena (DeJong, Liang, \& Lauber, 1994; Hommel, 1993; Lu, 1997; Virzi, \& Egeth, 1985), along with several neural network models (Kornblum, et al., 1999; Zorzi, \& Umilta, 1995). 
Inverse priming effects with response-compatible stimuli might rely on this automatic translation route. Only if prime stimuli vary along a dimension overlapping with the response set, the corresponding response is automatically activated. This initial prime-induced activation might then be suppressed by a motor-inhibition mechanism due to mask presentation (Jaśkowski, \& Przekoracka-Krawczyk, 2005; Lleras, \& Enns, 2006; Schlaghecken, \& Eimer, 2002). However, almost all studies on inverse priming used (double) arrow stimuli which constitute one example for response-compatible stimuli for left- and right-hand responses. Apart from the response compatibility, arrows are over-learned indicators for the concepts of left and right. Maybe this inherent spatial significance is the crucial feature of arrow stimuli and not their response-compatibility.

\subsection{Are arrows special or does inverse priming result with all response-compatible stimuli?}

Kornblum et al. (1990) did not differentiate between arrows and other response-compatible stimuli in their dimensional overlap model but when irrelevant masks are employed we demonstrated inverse priming only with arrow stimuli (Project III). Nevertheless, responsecompatibility seems to be the deceisive feature of arrows in the context of inverse priming for two reasons. First, some studies demonstrated inverse priming with non-arrow, but responsecompatible stimuli, under conditions ruling out a simple perceptual prime-mask interaction as the source of the effect (Jaśkowski, \& Ślósarek, 2007; Schlaghecken, et al., 2007; Verleger, et al., 2005). Second, inverse priming effects with arrow stimuli are completely unaffected by a reversal of SR-mappings (Jaśkowski, \& Ślósarek, 2007; O’Connor, \& Neill, 2011). When a leftward pointing arrow requires a right-hand response (and vice versa) response speed decreases, but importantly inverse priming is not altered.

Response-compatibility seems to be a necessary factor for inverse priming (with irrelevant masks) but in itself it is not sufficient (Project III, Experiment 5-7). Arrows might furthermore be especially suited to demonstrate inverse priming because they constitute objects (in contrast to an assembly of isolated visual features) which might be processed as entities. However, future research has to demonstrate whether other non-arrow objects also reliably produce inverse priming. Further insights might be expected from the use of human face stimuli which are known to be processed holistically (Robbins, \& McKone, 2003; Schiltz, \& Rossion, 2006). Similar to arrows (Tipples, 2008), eye gaze direction within such face stimuli has been proved to elicit automatic shifts of spatial attention (Frisen, Ristic, \& Kingstone, 
2004; Kuhn, \& Kingstone, 2009) even when such stimuli remained unconscious by masking (Sato, Okada, \& Toichi, 2007).

\subsection{Inverse priming with response non-compatible stimuli}

While response-compatible stimuli produced inverse priming with both types of masks, response non-compatible stimuli led to stable effects only with relevant masks (Project I, and IV, Eimer, 1999; Jaśkowski, \& Ślósarek, 2007; Kiesel, et al., 2008; Mattler, 2005; 2006; 2007) whereas the effect was absent with irrelevant masks (Project III; Jaśkowski, \& Ślósarek, 2007; Klauer, \& Dittrich, 2010; Kiesel, et al., 2008). There is ample evidence for a purely perceptual origin of the effect when relevant masks are employed together with response non-compatible stimuli. Two independent variants of the PRP-paradigm where applied to localize the inverse priming effect with such stimuli. Consistently, a perceptual locus was found in both experiments (Project I). Along the same lines, Mattler (2006) disentangled truly congruency-related effects from effects of the visual similarity of primes and targets and found that the entire effect was due to the visual similarity. Furthermore, a perceptual origin would readily account for inverse non-motor priming (Mattler, 2006; 2007).

When relevant masks are used along with response non-compatible stimuli, the effect seems to result from the perceptual interplay of primes and masks rendering prime-incongruent mask features more salient (Lleras, \& Enns, 2004) which in turn facilitates perceptual processing of incongruent targets. Such a perceptual effect seems to be restricted to masks that allow easy extraction of task-relevant features from the mask. Such features seem to be especially salient in simple superposition masks (Project II) and probably also in metacontrast masks (Project I, Project IV, Experiment 1; Mattler, 2007). In more complex random line masks, perceptual learning might also lead to extraction of target features from the mask structure yielding mask-induced inverse priming (Przekoracka-Krawczyk, \& Jaśkowski, 2007).

Other perceptual theories of inverse priming like the perceptual habituation account (Huber, 2008) or the idea of a congruency-related modulation of attention (Sohrabi, \& West, 2009) seem to be ruled out as an explanation for this effect because then, a comparable inverse priming effect should also occur with irrelevant masks. However, with such masks, results were divergent and evidence for inverse priming is rather small. In accordance with previous data (Jaśkowski, \& Ślósarek, 2007; Klauer, \& Dittrich, 2010; Kiesel, et al., 2008), inverse priming effects completely disappeared in several motor priming experiments which 
combined irrelevant masks with response non-compatible stimuli (Project III). In contrast, a small but statistically significant effect was detected in two independent experiments employing a non-motor priming paradigm (Project IV) which seems to rely on the same mechanism as inverse motor priming (Mattler, 2006; 2007). This residual non-motor priming effect was only found among those subjects being unable to consciously perceive the primes. The inconsistent results in motor and non-motor priming are discussed in the following summary section.

\subsection{Summary: At least two different mechanisms generating inverse priming}

Our results suggest that there are at least two different ways to obtain inverse priming effects. In most cases, inverse priming is due to the use of response-compatible stimuli which have the potential to automatically invoke motor responses. Then, inverse priming results because prime-induced response preparation is inhibited which is triggered by cognitive control operations detecting a mismatch of sensory evidence (resulting from processing of the masking stimulus) with the ongoing response preparation processes (Boy, et al., 2010; Boy, et al., 2009; Boy, \& Sumner, 2010; Jaśkowski, \& Przekoracka-Krawczyk, 2005; Jaśkowski, 2008; Lleras, \& Enns, 2006). When relevant masks are used, this process is further reinforced either because the mask-triggered inhibition is more effective with masks containing taskrelevant features (Jaśkowski, \& Verlerger, 2007) or because a perceptual prime-mask interaction produces active further support for the prime-incongruent response (mask-induced positive priming, Lleras, \& Enns, 2004; cf. subsection 3.6.). Such perceptual interactions also occur with non-compatible stimuli when a relevant mask is used. However, it does not automatically imply the translation to motor output due to the non-compatible nature of the stimuli used. Instead, as indicated by psychophysical evidence (Mattler, 2006; Krüger, et al., 2010) perceptual recognition of incongruent targets might be speeded up which constitutes the second way to achieve inverse priming effects.

This model, however, does not capture all instances of inverse priming. In Project IV, we observed evidence for inverse priming in a non-motor priming paradigm when irrelevant masks were used. If inverse priming with irrelevant masks is a pure motor phenomenon, resulting from the inhibition of the response directly activated by the prime, non-motor priming effects with irrelevant masks would not be expected because prime stimuli are not associated with any specific motor output. Alternatively, in non-motor priming, however, primes might automatically activate the assigned task sets in an analogous manner as motor 
responses are activated in motor priming. In the inverse non-motor priming paradigm employed in Project IV, for instance, the prime associated with the face discrimination task might activate task-related brain areas like e.g. the fusiform face area (Kanwisher, McDurmott, \& Chan, 1997) and the alternative prime areas involved in scene processing like e.g. the parahippocampal place area (Epstein, \& Kanwisher, 1998). Then, the processing of the masking stimulus might interrupt and even reverse this prime induced activity. Consequently, the mechanism responsible for inverse motor priming could theoretically be adapted to encompass inverse non-motor priming effects too. However, at least in the motor priming domain the important feature to trigger these processes leading to inverse priming was the response-compatibility of the primes. Similarly, in non-motor priming, primes could be compatible to the two alternative tasks in an abstract manner (cf. Footnote 1). If primes and tasks share some critical feature an automatic activation of task sets might be plausible. Although possible, the prime stimuli used, double arrows (Experiment 2) and vertically oriented arrows pointing either towards each other or away from each other (Experiment 3, see Figure 4), cannot be mapped onto the tasks in a natural way. Prime-task assignments seem arbitrary in this situation in contrast to SR-mappings for e.g. double arrows. Thus, since primes are not compatible to the two task alternatives in terms of an automatic activation of the task-related brain structures (like the motor system in inverse motor priming with response-compatible stimuli) inverse priming with irrelevant masks would not be expected. The evidence for inverse priming with such non-compatible primes contradicts the idea that inverse priming with irrelevant masks is exclusively a motor phenomenon which results from direct motor activations induced by response-compatible stimuli.

The idea that both, motor and non-motor priming, rely on common processing pathways is well established and supported by several common characteristics of both effects (Mattler, 2003; 2006; 2007). Accepting that both phenomena reflect the same underlying mechanism, the inconsistency could result from some obvious differences among the experiments. (1) In the non-motor priming paradigm, task practice was more extended, which is known to be a crucial factor for the generation of priming effects (Boy, \& Sumner, 2010; Klapp, \& Hinkley, 2002; Przekoracka-Krawczyk, \& Jaśkowski, 2007). (2) Both paradigms might have differed with respect to prime visibility, which has been found to moderate inverse priming effects (e.g. Klapp, 2005; Sumner, et al., 2006; Eimer, \& Schlaghecken, 2002). (3) Inverse priming effects are altered by overall response speed (Project III; Schlaghecken, \& Eimer, 2000; Maylor, et al., in press) which certainly differed between motor- and non-motor priming. 
(1) It is not likely that the differential amount of practice accounts for the absence of effects in the motor priming task, because in Experiment 9 of Project III we still failed to observe any effect even after five sessions of training (while subjects performed only three sessions in the non-motor priming paradigm). (2) Neither differences with respect to prime visibility seem to be able to explain the divergent results because average prime recognition performance was comparable in the non-motor priming experiments (overall $56 \%$ and $58 \%$ correctly reported primes, respectively) and motor priming experiments with non-arrow stimuli (range 52 -68 $\%)$. In particular, analyzing priming effects only among those subjects showing subliminal prime perception did not provide evidence for the effect in the motor priming paradigm. Thus, prime visibility also seems to be ruled out as a potential difference accounting for the divergent results. (3) Response speed is crucial for the magnitude of inverse priming effects potentially reflecting the temporal profile of response activation and inhibition processes (Schlaghecken, \& Eimer, 2000; Maylor, et al., in press; cf. LRP-results, e.g. Eimer, \& Schlaghecken, 1998). Nevertheless we still failed to find evidence for inverse priming with non-arrow stimuli when we searched for the effect at several different RT bins across the entire distribution. Particularly, even when considering only the slowest of all responses no effect became apparent (Krüger, \& Mattler, in prep, Appendix III). Thus, all hypotheses proposed above do not give a satisfying answer for why inverse effects were found in the nonmotor paradigm but not in the motor paradigm. Rather, differences with respect to practice, prime visibility or absolute response latencies seem to be excluded as potential factors explaining the divergent results.

Recently, similar to our non-motor priming effects, a residual inverse motor priming effect was reported under conditions ruling out perceptual prime-mask interactions and motor inhibition as the source (Klauer, \& Dittrich, 2010; Experiment 4). Perceptual interactions were prevented by presenting the stimulus sequence at very slow pace (prime-mask and masktarget SOA were $180 \mathrm{~ms}$ ) and by using a masking stimulus from a different stimulus family which is comparable to the use of an irrelevant mask. Similar to non-motor priming, primes were not associated with any motor output which was achieved by a trial-to-trial variation of SR-mappings. SR-mappings were signaled to the subject when targets were presented. Nonetheless, Klauer and Dittrich (2010) reported a residual inverse priming effect which they explained in the framework of their evaluation window account. According to this view, primes feed central accumulators representing the abstract response alternatives (which are unrelated from the representation of the response itself) biasing them before mask presentation triggers an evaluation monitoring process. Abstract response selection is based 
on the relative increase of evidence gathered during this evaluation window. Because the primed alternative already reached some non-zero state, increase rates are lower as compared to the unprimed alternative which is still in its initial state, thus facilitating selection of the unprimed, i.e. incongruent response, yielding inverse priming. This is due to diminishing increase rates with growing absolute evidence ('Weber-Fechner-Law', Miller, 1964). The evaluation window account relies on central response selection. Thus, it principally also provides an explanation for non-motor priming effects. Then, the two accumulators would represent the two alternative tasks.

If such a residual effect exists which does neither result from motor inhibition nor from perceptual prime-mask interactions, why did we fail to observe it in the motor priming paradigm (Project III, Experiment 2, 3, 5, 6, 7)? Possibly, the absence of inverse priming might be due to the experimental context. The probability for inverse priming to occur suffered from the inclusion of additional lateral targets which became apparent when comparing results from Experiment $4 \mathrm{a}$ and $4 \mathrm{~b}$. We only observed inverse priming in Experiment $4 \mathrm{~b}$ which employed two (central) targets in contrast to Experiment $4 \mathrm{a}$. Evidence for the view that additional targets reduce priming effects can also be derived from the comparison of inverse priming effects with double arrow stimuli. In Experiment 1 of Project III we obtained an effect of $23 \mathrm{~ms}$ when lateral targets were included. Omitting lateral targets, but combining the same mask with the identical double arrow stimuli (Project II, orthogonal mask condition) yielded an effect of $38 \mathrm{~ms}$. As an additional confounded difference, masktarget SOA varied in Project II. However, randomized presentation of several distinct SOAs does not affect inverse priming effects (see Krüger, \& Mattler, in prep., Appendix II). Thus, results suggest that inverse priming effects are actually reduced by the inclusion of lateral targets. The primes' influence might be limited when the target set is augmented, because prime stimuli only represent $50 \%$ of all possible outcomes. Consequently, restricting the target set might be a promising way to extract small but reliable inverse priming effects with non-compatible stimuli combined with irrelevant masks which might result from an additional central evidence monitoring mechanism.

Apart from the relevance of our results for the exploration of the mechanisms underlying inverse priming, our results also contribute to clarify two further important issues in the study of inverse priming effects: the role of prime visibility and the question whether effects of relevant masks and irrelevant masks rely on a shared mechanism. These issues will be addressed in the following two sections. 


\subsection{The role of prime visibility in inverse priming}

Initially, prime visibility has been considered as an important factor which determines the sign of priming effects. While subliminal primes produced inverse effects, supraliminal ones lead to positive priming (Eimer, \& Schlaghecken, 2002; Klapp, \& Hinkley, 2002). Subsequently, however, the claim of a strict causal relationship of prime visibility and priming effects has been relaxed (Schlaghecken, et al., 2007; Schlaghecken, et al., 2008) in the light of various studies demonstrating on the one hand inverse priming effects with clearly supraliminal primes (Jaśkowski, 2007; 2008; Jaśkowski, et al., 2008; Klapp, 2005; Lleras, \& Enns, 2005; 2006; Sumner, et al., 2006) and on the other hand positive priming across the entire range of prime visibility (cf. Lleras, \& Enns, 2006). Therefore, prime invisibility cannot be considered a prerequisite for inverse priming to occur. Nonetheless, both, priming effects and prime visibility, are affected by various factors at the same time, like prime duration (Eimer, \& Schlaghecken, 2002; Schlaghecken, et al., 2007; Sumner, et al., 2006), mask density (Eimer, \& Schlaghecken, 2002) or the prime-mask SOA (Lleras, \& Enns, 2004) suggesting that both are related to each other. To exclude such confounded factors as a potential cause for a relation, prime recognition has to be altered while other factors have to be kept constant. Thus, exploiting the natural variability of the individual capacity to recognize the primes provides insights into potential relations between prime visibility and priming effects.

The presented experiments revealed that priming effects were independent of prime visibility when relevant masks were used irrespective of whether stimuli were response-compatible (Project II, Project V: correlation of priming effects and prime visibility across participants $\mathrm{r}$ $=.11, \mathrm{p}=.63$ ) or response non-compatible (Project I, see Krüger, et al., 2011; Project IV). As reported above, data from experiments which employed irrelevant masks suggested a stable relationship (Project II, III, IV, V). Data from 142 participants of all those experiments which employed irrelevant masks and yielded inverse priming were reanalyzed. Priming effects and prime recognition performance co-varied $(r=.40, \mathrm{p}<.0001$; see Figure 6A). Inverse effects were accompanied by poor prime recognition performance and increasing recognition performance led to deteriorating inverse effects. Next, subjects showing sub- and supraliminal prime perception were considered in isolation (Figure 6B). Determining a threshold for conscious prime perception is necessarily arbitrary. Three approaches were made which yield similar results. First, equally sized groups were built by dividing the sample according to the median of prime recognition performance $(51.7 \%$ correctly reported primes, cf. Mattler, 2007). Second, assuming that a subject is guessing on the recognition task the proportion of 
correctly reported primes should follow a binomial distribution $(\mathrm{N}=$ number of trials, $\mathrm{p}=$ $0.5)$. We calculated the criterion based on the minimum number of trials we employed in the prime recognition session $(\mathrm{N}=576)$. The probability that a subject guessed is below $5 \%$ when performance exceeds $53.5 \%$ correctly reported primes which was taken as the second criterion (cf. Krüger, \& Mattler, in prep., Appendix III). Third, prime visibility data was subjected to a $\mathrm{k}$-means cluster analysis for $\mathrm{k}=2$ groups. Optimal separation of two groups according to prime recognition performance is achieved at $65.6 \%$ (mean of maximum in the poor performance group and minimum in the good performance group).

Thus, we derived three criteria to divide the complete sample into subjects performing good versus poor on the prime recognition task. For all three criteria, inverse priming effects differed across the two groups defined by prime recognition performance irrespective of whether the division followed the median-split criterion, $\mathrm{t}(140)=5.3, \mathrm{p}<.0001$, the binomial distribution criterion, $\mathrm{t}(140)=5.2, \mathrm{p}<.0001$, or the cluster-analytical criterion, $\mathrm{t}(140)=3.4, \mathrm{p}$ $<.001$ (cf. Figure 6B). In either case, subjects with poor recognition performance showed comparatively strong inverse priming effects $(-30 \mathrm{~ms}, \mathrm{t}(70)=-9.2, \mathrm{p}<.0001,-28 \mathrm{~ms}, \mathrm{t}(78)=-$ $8.8, \mathrm{p}<.0001,-22 \mathrm{~ms}, \mathrm{t}(119)=-8.6, \mathrm{p}<.0001$, for the three criteria, respectively). In contrast those subjects showing supraliminal performance showed rather small inverse priming effects according to the median-split criterion $(-7 \mathrm{~ms}, \mathrm{t}(70)=-2.6, \mathrm{p}<.05)$ and the binomial distribution criterion $(-6 \mathrm{~ms}, \mathrm{t}(62)=-2.3, \mathrm{p}<.05)$, and even no significant effect according to the cluster-analytical criterion $(-1 \mathrm{~ms}, \mathrm{t}(21)=0.2, \mathrm{p}=.85)$.

These results are in line with similar analyses performed by Klapp (Klapp, \& Hinkley, 2002; Klapp, 2005) and with observations reported by Sumner (2007). In contrast to inverse priming with irrelevant masks, positive priming effects seem to be largely independent of prime visibility (Vorberg, et al., 2003; Mattler, 2003). In the context of inverse priming, effects might depend upon visibility because prime visibility might affect the amount of primeinduced activation (at least for response-compatible stimuli). Better visibility might lead to stronger activation of the corresponding response and mask-triggered inhibition might never fully overcome this stronger initial prime-induced activation leading to smaller inverse effects (see also Sumner, 2007). 

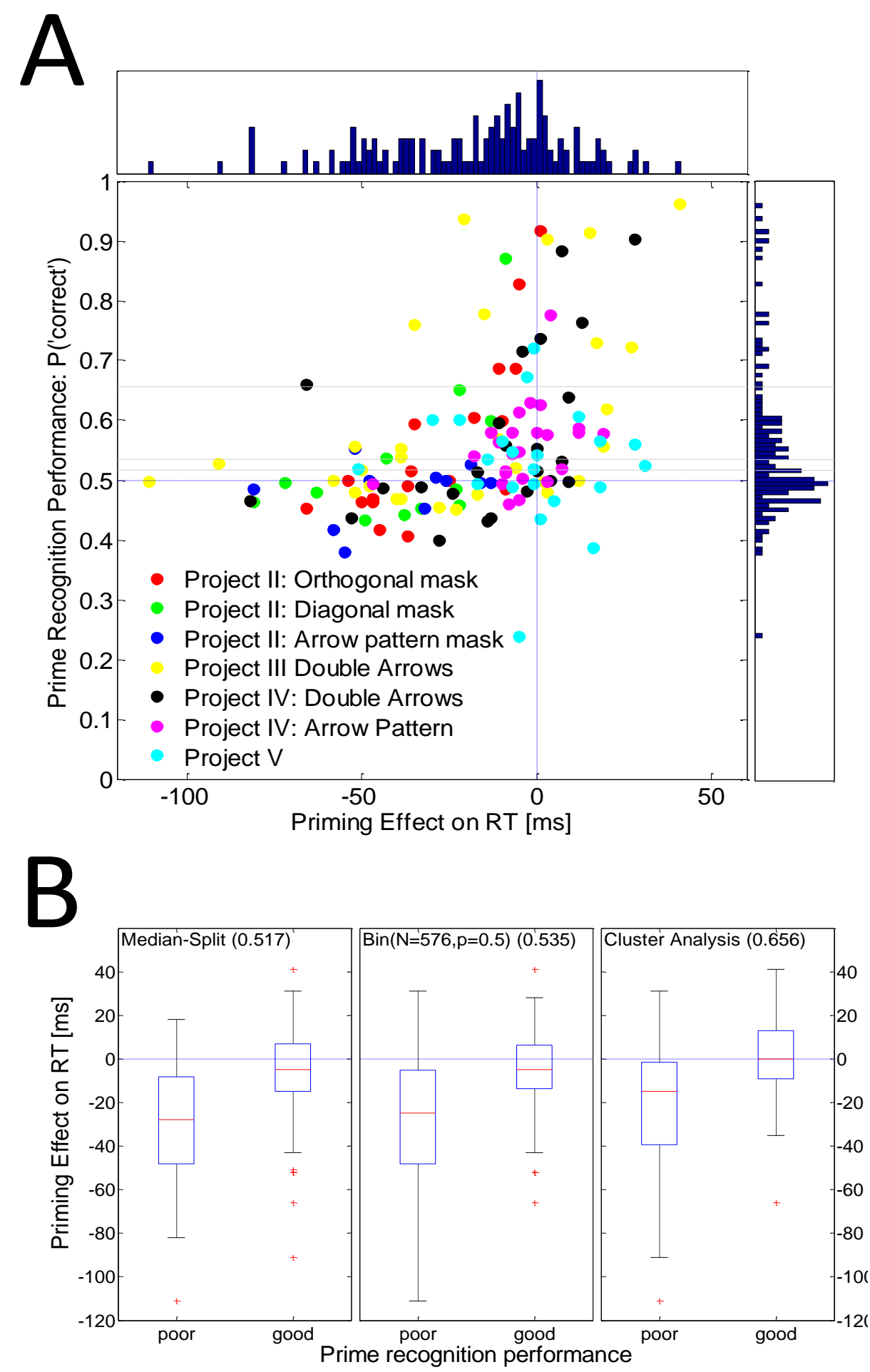

Figure 6. Reanalysis of prime recognition performance and priming effects with irrelevant masks. A) Prime recognition performance and priming effect data from 142 subjects are depicted from seven different experiments. Common to all these experiments, the mask-target SOA (or mask-cue SOA, respectively) was 150 ms. For all target-priming experiments (Project II, III, V), double arrows were employed as prime stimuli. In contrast, in the second non-motor priming experiment (Project IV) primes were arrow pattern compositions. Priming effects and prime recognition performance covaried $(r=.40, \mathrm{p}<.0001)$. Thin grey lines refer to three different criteria to distinguish subliminal from supraliminal prime recognition performance (see text). B) Box plots of priming effects of the subgroups with good and poor prime recognition performance for the three different divisions. Priming effects differed between those subjects showing poor prime recognition versus good prime recognition performance irrespective of the criterion considered. 


\subsection{A common mechanism of inverse priming with relevant and irrelevant masks}

Apart from the role of prime visibility in inverse priming, another unresolved issue is whether two completely distinct mechanisms produce inverse priming with relevant and irrelevant masks (Klapp, 2005) or whether a common mechanism is involved with both types of masks (Jaśkowski, \& Verlerger, 2007). For response non-compatible stimuli such a shared mechanism for both masks seems to be ruled out. If inverse priming with such stimuli and irrelevant masks exists at all (Project IV, Klauer, \& Dittrich, 2010), the mechanism producing it is certainly distinct from the perceptual effect found with relevant masks (Project I; Mattler, 2006; 2007). For response-compatible stimuli three approaches seem to be possible, accounting for large effects with relevant and reduced effects with irrelevant masks (Krüger, et al., 2011). First, the efficiency of a single mechanism is modulated by the mask structure ('Single-Mechanism Approach'). Thus, mask triggered motor inhibition is more effective when the mask entails task-relevant elements (Verlerger, \& Jaśkowski, 2007). Second, two mechanisms jointly produce inverse priming with relevant and irrelevant masks ('Co-active Mechanisms Approach'). Mask triggered inhibition is equally effective with both types of masks producing a basic part of the effect while perceptual prime-mask interactions further enlarge the effect with relevant masks (Lleras, \& Enns, 2006; Schlaghecken, et al., 2007). As suggested by previous results (Boy, \& Sumner, 2010; Eimer, 1999; Klapp, \& Hinkley, 2002; O'Connor, \& Neill, 2011; Schlaghecken, \& Eimer, 2004), this additional mechanism specifically involved with relevant masks should then also affect motor processes. As proposed by Lleras and Enns (2004), perceptual processes might be directly linked to the motor system such that the perceptual prime-mask interaction activates the prime-incongruent response independent of ongoing mask-triggered inhibition of the primed response. Third, one mechanism generates inverse priming with relevant masks while a completely different one independently produces the effect with irrelevant masks ('Separate-Mechanisms Approach', Klapp, 2005). Then, mask-triggered inhibition of the prime evoked motor activity would be restricted to irrelevant masks. In contrast, the effect with relevant masks might completely result from prime-incongruent motor activity induced by the more salient (i.e. incongruent) parts of the mask.

It might seem counterintuitive that one mechanism generating the effect with irrelevant masks is completely ineffective when the mask is replaced by a relevant one, but the evidence gathered so far supports the Separate-Mechanisms Approach. First, the differential role of prime visibility for inverse priming effects suggests independent mechanisms. While inverse effects increase as masking becomes more efficient with irrelevant masks, no comparable 
relation was found for relevant masks. A reliable influence of prime visibility on priming effects with irrelevant masks which is completely absent with relevant masks contradicts the notion of a common mechanism producing inverse priming with both types of masks. Along the same lines, Klapp (2005) reanalyzed data from the relevant mask conditions provided by Lleras and Enns (2004) and even argued for an opposite influence of prime visibility in case of relevant and irrelevant masks. According to his view, inverse effects grow with increasing prime visibility for relevant masks. Second, inverse priming with relevant and irrelevant mask has different underlying time-courses (cf. Figure 2 from Krüger, \& Mattler, in prep., Appendix II). With relevant masks, effects peaked at $100 \mathrm{~ms}$ declined when mask-target SOA was further increased. In contrast, effects with irrelevant masks were of a comparable magnitude at a 100 and $150 \mathrm{~ms}$ SOA condition.

In contrast to these observations, we found a common neural correlate of control operations in inverse priming for both types of masks as revealed by our MRI investigation (Project V). We extended previous evidence for a crucial role of the SMA in inverse priming with irrelevant masks to relevant masks. In addition, several further brain areas were shown to be specifically involved when relevant masks were used. These results could be taken as evidence for a common mechanism generating inverse priming with both types of masks. However, separate mechanisms could also rely on largely distinct neural networks which include the SMA as a common module.

Taken together, current evidence seems to favour two completely distinct mechanisms involved in inverse priming with relevant and irrelevant masks for both, response-compatible and response non-compatible stimuli. However, future research is needed to shed further light on this question.

\subsection{Conclusion}

The empirical studies conducted suggest that inverse priming is a rather multifaceted phenomenon. The inverse priming effect can be observed with various types of stimuli. However, the underlying mechanisms seem to differ. Inverse priming seems to arise from motor levels of processing when response-compatible stimuli are used irrespective of the mask employed. In contrast, non-compatible stimuli produce unequivocal effects only when relevant masks are used. Then, the effect originates from perceptual processes. It remains unclear whether inverse priming is also possible with such non-compatible stimuli when 
irrelevant masks are employed. Residual effects could be accounted for by contributions at response selection levels. For the investigation of inverse priming, these inferences imply that experimental findings have to be interpreted with caution because generalizability of findings might be limited to the current experimental situation.

Moreover, the phenomenon of inverse priming illustrates that processing of subliminal stimuli goes beyond the automatic activation of motor responses. The complex pattern of results in the reported experiments provides further support for the view that perceptuo-motor processing of subliminal stimuli in not a simple bottom-up process. Instead, several control processes seem to operate outside of awareness continuously monitoring for mismatches of currently incoming perceptual input and ongoing motor preparation processes in order to adapt response preparation to changing task demands. 


\section{References}

Albrecht, T., Klapötke, S., \& Mattler, U. (2010). Individual differences in metacontrast masking are enhanced by perceptual learning. Consciousness \& Cognition, 19(2), 656-666.

Ansorge, U., Becker, S.I., \& Breitmeyer, B. (2009). Revisiting the metacontrast dissociation: Comparing sensitivity across different measures and tasks. The Quarterly Journal of Experimental Psychology, 62 (2), 286-309.

Aron, A.R., Schlaghecken, F., Fletcher, P.C., Bullmore, E.T., Eimer, M., Barker, R., Sahakian, B.I., \& Robbins, T.W. (2003). Inhibition of subliminally primed responses is mediated by the caudate and thalamus: evidence from functional MRI and Huntington's disease. Brain, 126, 713-723.

Berti, A., \& Rizzolatti, G. (1992). Visual processing without awareness: evidence from unilateral neglect. Journal of Cognitive Neuroscience, 4 (4), 345-351.

Bodner, G.E., \& Masson, D.E. (2003). Beyond spreading activation: An influence of relatedness proportion on masked semantic priming. Psychonomic Bulletin and Review, $10,645-652$.

Boy, F., Clarke, K., \& Sumner, P. (2008). Mask stimulus triggers inhibition in subliminal visuomotor priming. Experimental Brain research, 190, 111-116.

Boy, F., Evans, C.J., Edden, R.A.E., Singh, K.D., Husain, M., \& Sumner, P. (2010). Individual differences in subconscious motor control predicted by GABA concentration in SMA. Current Biology, 20, 1779-1785.

Boy, F. Husain, M., Singh, K.D., \& Sumner, P. (2010). Supplementary motor area activations in unconscious inhibition of voluntary action. Experimental Brain Research, 206, 441-448.

Boy, F., \& Sumner, P. (2010). Tight coupling between positive and reversed priming in the masked priming paradigm. Journal of Experimental Psychology: Human Perception and Performance, 36(4), 892-905.

Breitmeyer, B. G. (1984). Visual masking: An integrative approach. Oxford: Clarendon Press. 
Breitmeyer, B. G., \& Ögmen, H. (2006). Visual Masking: Time Slices through Conscious and Unconscious Vision (Second Ed.). New York: Oxford University Press.

Chen, X., Scangos, K.W., Stuphorn, V. (2010). Supplementary motor area exerts proactive and reactive control of arm movements. The Journal of Neuroscience, 30(44), 1465714675.

Cole, G.C., \& Kuhn, G. (2010). What the experimenter's prime tells the observer's brain. Attention, Perception, \& Psychophysics, 72 (5), 1367-76.

Colebatch, J.G. (2007). Bereitschaftspotential and movement-related potentials: origin, significance, and application in disorders of human movement. Movement Disorders, 22(5), 601-610.

De Jong, R., Liang, C.-C., \& Lauber, E. (1994). Conditional and unconditional automaticity: A dual-process model of effects of spatial stimulus-response correspondence. Journal of Experimental Psychology: Human Perception and Performance, 20, 731-750.

Derbyshire, N., Ellis, R., \& Tucker, M. (2006). The potentiation of two components of the reach-to-grasp action during object categorisation in visual memory. Acta Psychologica, $122,74-98$.

D’Ostilio, K., \& Garraux, G. (2011). Automatic stimulus-induced medial premotor cortex activation without perception or action. PloS ONE, 6 (2): e16613.

Eimer, M. (1993). Spatial cuing, sensory gating and selective response preparation: an ERPstudy on visuo-spatial orienting. Electroencephalography and clinical Neurophysiology, $88,408-420$.

Eimer, M. (1995). Stimulus-response compatibility and automatic response activation: evidence from psychophysiological studies. Journal of Experimental Psychology: Human Perception and Performance, 21(4), 837-854.

Eimer, M. (1999). Facilitatory and inhibitory effects of masked prime stimuli on motor activation and behavioural performance. Acta Psychologica, 101, 293-313.

Eimer, M., Hommel, B., \& Prinz, W. (1995). S-R compatibility and response selection. Acta Psychologica, 90, 301-313. 
Eimer, M. \& Schlaghecken, F. (1998). Effects of masked stimuli on motor activation: Behavioral and electrophysiological evidence. Journal of Experimental Psychology: Human Perception and Performance, 24, 1737-1747.

Eimer, M., \& Schlaghecken, F. (2002). Links between conscious awareness and response inhibition: Evidence from masked priming. Psychonomic Bulletin \& Review, 9, 514-520.

Eimer, M., \& Schlaghecken, F. (2003). Response facilitation and inhibition in subliminal priming. Biological Psychology, 64, 7-26.

Eimer, M. \& Schubö, A., \& Schlaghecken, F. (2002). Locus of inhibition in the masked priming of response alternatives. Journal of Motor Behaviour, 34, 3-10.

Epstein, R., \& Kanwisher, N. (1998). A cortical representation of the local visual environment. Nature, 392,598-601.

Friesen, C.K., Ristic, J., \& Kingstone, A. (2004). Attentional effects of counterpredictive gaze and arrow cues. Journal of Experimental Psychology: Human Perception and Performance, 30, 319-329.

Georgopoulos, A.P., Lupito, J.T., Petrides, M., Schwartz, A.B., \& Massey, J.T. (1989). Mental rotation of the neuronal population vector. Science, 243, 234-236.

Goebel, R., Khorram-Sefat, D., Muckli, L., Hacker, H., \& Singer, W. (1998). The constructive nature of vision: direct evidence from functional magnetic resonance imaging studies of apparent motion and motion imagery. European Journal of Neuroscience, 10, 1563-1573.

Guzzon, D., Brignani, D., Miniussi, C., \& Marzi, C.A. (2010). Orienting of attention with eye and arrow cues and the effect of overtraining. Acta Psychologica, 134 (3), 353-362.

Jaśkowski, P. (2007). The effect of nonmasking distractors on the priming of motor responses. Journal of Experimental Psychology: Human Perception and Performance, 33, 456-468.

Jaśkowski, P. (2008). The negative compatibility effect with nonmasking flankers: A case for mask-triggered inhibition hypothesis. Consciousness and Cognition, 17, 765-777. 
Jaśkowski, P., Biłuńska, A., Tomanek, M., \& Verleger, R. (2008). Mask- and distractortriggerd inhibitory processes in the priming of motor responses: An EEG study. Psychophysiology, 45, 70-85.

Jaśkowski, P., \& Przekoracka-Krawczyk, A. (2005). On the role of mask structure in subliminal priming. Acta Neurobiologiae Experimentalis, 65, 409-417.

Jaśkowski, P., \& Ślósarek, M. (2007). How important is the prime's gestalt for subliminal priming? Consciousness \& Cognition, 16, 485-497.

Jaśkowski, P., \& Verleger, R. (2007). What determines the direction of subliminal priming. Advances in Cognitive Psychology, 3 (1-2), 181-192.

Goodale, M.A., Milner, A.D., Jakobsen, L.S., \& Carey, D.P. (1991). A neurological dissociation between perceiving objects and grasping them. Nature, 349, 154-156.

Hazeltine, E., Poldrack, R., \& Gabrieli, J.D.E. (2000). Neural activation during response conflict. Journal of Cognitive Neuoscience, 12, 118-129.

Hellige, J.B., Walsh, D.A., Lawrance, V.W., \& Prasse, M. (1979). Figural relationship effects and mechanisms of visual masking. Journal of Experimental Psychology: Human Perception and Performance, 5, 88-100.

Hommel, B. (1993). The relationship between stimulus processing and response selection in the Simon task: Evidence for a temporal overlap. Psychological Research, 55, 280-290.

Huber, D.E. (2008). Immediate priming and cognitive aftereffects. Journal of Experimental Psychology: General, 137, 324-347.

Kanwisher, N., McDermott, J., \& Chun, M.M. (1997). The fusiform face area: A modul in human extrastriate cortex specialized for face perception. The Journal of Neuroscience, $11,4302-4311$.

Kato, Y., Endo, H., Kizuka, T., \& Asami, T. (2006). Automatic and imperative motor activations in stimulus-response compatibility: magnetoencephalographic analysis of upper and lower limbs. Experimental Brain Research, 168 (1-2), 51-61.

Kiefer, M., \& Martens, U., (2010). Attentional sensitization of unconscious cognition: Task sets modulate subsequent masked semantic priming. Journal of Experimental Psychology: General, 139 (3), 464-489. 
Kiesel, A., Berner, M.P., \& Kunde, W. (2008). Negative congruency effects: A test of the inhibition account. Consciousness and Cognition, 17, 1-21.

Kinsbourne, M., \& Warrington, E.K. (1962). Further studies on the masking of brief visual stimuli by a random pattern. Quarterly Journal of Experimental Psychology, 14, 235-245.

Klapötke, S., Krüger, D., \& Mattler, U. (2011). A PRP-study to determine the locus of target priming effects. Consciousness and Cognition, 20, 882-890.

Klapp, S. T. (2005). Two versions of the negative compatibility effect: Comment on Lleras and Enns (2004). Journal of Experimental Psychology: General, 134, 431-435.

Klapp, S. T., \& Haas, B. W. (2005). Nonconscious influence of masked stimuli on response selection is limited to concrete stimulus-response associations. Journal of Experimental Psychology: Human Perception and Performance, 31, 93-209.

Klapp, S. T., \& Hinkley, L. B. (2002). The negative compatibility effect: Unconscious inhibition influences reaction time and response selection. Journal of Experimental Psychology: General, 131, 255-269.

Klauer, K.C., \& Dittrich, K. (2010). From sunshine to double arrows: An evaluation window account of negative compatibility effects. Journal of Experimental Psychology: General, $139,3,490-519$.

Klotz, W. \& Neumann, O. (1999). Motor activation without conscious discrimination in metacontrast masking. Journal of Experimental Psychology: Human Perception and Peformance, 25, 976-992.

Kornblum, S., Hasbroucq, T., \& Osman, A. (1990). Dimensional overlap: cognitive basis for stimulus-response compatibility - A model and taxonomy. Psychological Review, 97(2), 253-270.

Kornblum, S., Stevens, G, Whipple, A., and Requin, J. (1999). The effects of irrelevant stimuli: 1. The time-course of stimulus-stimulus and stimulus-response consistency effects with Stroop-like stimuli, Simon-like tasks, and their factorial combinations. Journal of Experimental Psychology: Human Perception and Performance, 25, 688-714. 
Kouider, S. \& Dehaene, S. (2007). Levels of processing during non-conscious perception: a critical review of visual masking. Philosophical Transactions of the Royal Society of London. Series B, Biological Sciences, 362, 857-875.

Krüger, D., Klapötke, S., \& Mattler, U. (2011). PRP-paradigm provides evidence for a perceptual origin of the negative compatibility effect. Consciousness and Cognition, 20, 866-881.

Kuhn, G., \& Kingstone, A. (2009). Look away! Eyes and arrows engage oculomotor responses automatically. Attention, Perception, \& Psychophysics, 71(2), 314-327.

Leuthold, H. (2011). The simon effect in cognitive electrophysiology: A short review. Acta Psychologicy, 136, 203-211.

Lleras, A. \& Enns, J.T. (2004). Negative compatibility or object updating? A cautionary tale of mask-dependent priming. Journal of Experimental Psychology: General, 133, 475-493.

Lleras, A. \& Enns, J.T. (2005). Updating a cautionary tale of masked priming: Reply to Klapp (2005). Journal of Experimental Psychology: General, 134, 436-440.

Lleras, A., \& Enns, J. T. (2006). How much like a target can a mask be? Geometric, spatial, and temporal similarity in priming: A reply to Schlaghecken and Eimer (2006). Journal of Experimental Psychology: General, 135, 495-500.

Lu, C.-H. (1997). Correspondence effects for irrelevant information in choice-reaction tasks: Characterizing the stimulus-response relations and the processing dynamics. In $\mathrm{B}$. Hommel \& W. Prinz (Eds.), Theoretical issues in stimulus-response compatibility (pp. 85- 117). Amsterdam: Elsevier.

Malikovic, A., Amunts, K., Schleicher, A., Mohlberg, H., Eickhoff, S.B., Wilms, M., Palomero-Gallagher, N., Armstrong, E., Zilles, K. (2007). Cytoarchitectonic Analysis of the Human Extrastriate Cortex in the Region of V5/MT1: A Probabilistic, Stereotaxic Map of Area hOc5. Cerebral Cortex, 17, 562-574.

Martens, S., \& Wyble, B. (2010). The attentional blink: past, present, and future of a blind spot in perceptual awareness. Neuroscience and Biobehavioral Reviews, 34(6), 947-957.

Mattler, U. (2003). Priming of mental operations by masked stimuli. Perception and Pschophysics, 65, 167-187. 
Mattler, U. (2005). Inhibition and decay of motor and non-motor priming. Perception and Pschophysics, 67, 285-300.

Mattler, U. (2006). On the locus of priming and inverse priming effects. Perception and Psychophysics, 68, 975-991.

Mattler, U. (2007). Inverse target- and cue-priming effects of masked stimuli. Journal of Experimental Psychology: Human Perception and Performance, 33, 83-102.

Maylor, E.A., Birak, K.S., \& Schlaghecken, F. (2011). Inhibitory control in old age: evidence for de-automization? Frontiers in Psychology, 2, 132-145.

McClelland, J. L. (1979). On the time relations of mental processes: A framework for analyzing processes in cascade. Psychological Review,86, 287-330.

Miller, G.A. (1964). Mathematics and psychology. New York, NY: Wiley.

Miller, J.O., \& Franz, E.A. (2005). Dissociation of bimanual responses with the simon effect: on the nonunitization of bimanual responses. Journal of Motor Behaviour, 37, 146-156.

Milner, A. D., \& Goodale, M. A. (1995). The visual brain in action. Oxford: Oxford University Press.

Milner, A. D., \& Goodale, M. A. (2006). The visual brain in action (Second Ed.). Oxford: Oxford University Press.

Milner, A.D., Perrett, D.I., Johnston, R.S., Beson, P.J., Jordan, T.R., Heeley, D.W., Bettucci, D., Mortara, F., Mutani, R., Terazzi, E., \& Davidson, D.L.W. (1991). Perception and action in visual form agnosia. Brain, 114, 405-428.

Nachev, P., Kennard, C., \& Husain, M. (2008). Functional role of the supplementary and presupplementary motor areas. Nature Reviews Neuroscience, 9, 856-869.

Neumann, O., \& Klotz, W. (1994). Motor responses to nonreportable, masked stimuli: Where is the limit of direct parameter specification? In C. Umiltà \& M. Moscovitch (Eds.), Attention and performance $X V$ : Conscious and nonconscious information processing (pp. 123-150). Cambridge,Mass.: MIT Press. 
O’Connor, P., \& Neill, W.T. (2011). Does subliminal priming of free response choices depend on task set or automatic response activation. Consciousness and Cognition, 20, 280-287.

Pashler, H., \& Johnston, J. C. (1998). Attentional limitations in dual-task performance. In H. Pashler (Ed.), Attention (pp. 155-189). Hove, England, UK: Psychology Press/Erlbaum Taylor \& Francis.

Picard, N., \& Strick, P.L. (1996). Motor areas of the medial wall: a review of their location and function. Cerebral Cortex, 6, 342-353.

Pohl, C., Kiesel, A., Kunde, W., \& Hoffmann, J. (2010). Early and late selection in unconscious information processing. Journal of Experimental Psychology: Human Perception and Performance, 36, 268-285.

Praamstra, P., \& Seiss, E. (2005). The neurophysiology of response competition: motor cortex activation and inhibition following subliminal response priming. Journal of Cognitive Neuroscience, 17 (3), 483-493.

Prinz, W. (1997). Perception and action planning. European Journal of Cognitive Psychology, 9(2), 129-154.

Procter, R.W., Lu.C.-H., Wang, H., \& Dutta, A. (1995). Activation of response codes by relevant and irrelevant stimulus information. Acta Psychologica, 90, 275-286.

Przekoracka-Krawczyk, A., \& Jaśkowski, P. (2007). Perceptual learning can reverse subliminal priming effects. Perception \& Psychophysics, 69 (4), 558-566.

Reingold, E. M. \& Merikle, P. M. (1988). Using direct and indirect measures to study perception without awareness. Perception \& Psychophysics, 44(6), 563-575.

Reingold, E. M. \& Merikle, P. M. (1993). Theory and measurement in the study of unconscious processes. In M. Davies \& G. W. Humphreys (Eds.), Consciousness: Psychological and philosophical essays (pp. 44-57). Oxford: Blackwell.

Ridderinkhof, K.R., van Wildenberg, W.P.M., Segalowitz, S.J., \& Carter, C.S. (2004). Neurocognitive mechanisms of cognitive control: The role of prefrontal cortex in action selection, response inhibition, performance monitoring, and reward-based learning. Brain and Cognition, 56, 129-140. 
Sanders, A. F. (1980). Stage analysis of reaction processes. In G. E. Stelmach \& J. Requin (Eds.), Tutorials in motor behavior (pp. 331-354). Amsterdam: North-Holland.

Sato, W., Okada, T., \& Toichi, M. (2007). Attentional shift by gaze is triggered without awareness. Experimental Brain Research, 183, 87-94.

Schiltz, C., \& Rossion, B. (2006). Faces are represented holistically in the human occipitotemporal cortex. Neuroimage, 32, 1385-1394.

Schlaghecken, F., Birak, K.S., \& Maylor, E.A. (in press). Age-related deficits in low-level motor inhibitory motor control. Psychology and Aging.

Schlaghecken F., Blagrove E., \& Maylor, E.A. (2008). No difference between conscious and nonconscious visuomotor control: evidence from perceptual learning in the masked prime task. Consciousness and Cognition. 17(1), 84-93.

Schlaghecken, F., Bowman, H., \& Eimer, M. (2006). Dissociating local and global levels of perceptuo-motor control in masked priming. Journal of Experimental Psychology: Human Perception and Performance, 32, 618-632.

Schlaghecken, F., \& Eimer, M. (2000). A central-peripheral asymmetry in masked priming. Perception \& Psychophysics, 62, 1367-1382.

Schlaghecken, F. \& Eimer, M. (2002). Motor activation with and without inhibition: evidence for a treshold mechanism in motor control. Perception and Psychophysics, 64, 148-162.

Schlaghecken, F., \& Eimer, M. (2006). Active masks and active inhibition: A comment on Lleras and Enns (2004) and on Verleger, Jaśkowski, Aydemir, van der Lubbe, and Groen (2004). Journal of Experimental Psychology. General, 135, 484-494.

Schlaghecken, F., Klapp, S.T., \& Maylor, E.A. (2009). Either or neither, but not both: locating the effects of masked primes. Proceedings of the Royal Society: Biological Sciences, 276, 515-521.

Schlaghecken, F., Maylor, E.A. (2005). Motor control in old age: Evidence for impaired lowlevel inhibition. Journal of Gerontology: Psychological Sciences, 60 (3), 158-161. 
Schlaghecken, F., Münchau, A., Bloem, B., Rothwell, J.C., \& Eimer, M. (2003). Slow frequency repetitive transcranial magnetic stimulation affects reaction times, but not priming effects, in a masked prime task. Clinical Neurophysiology, 14, 1272-77.

Schlaghecken, F., Rowley, L., Sembi, S., Simmons, R., \& Whitcomb, D. (2007). The negative compatibility effect: A case for self-inhibition. Advances in Cognitive Psychology, 3, 227-240.

Schmidt, T. \& Vorberg, D. (2006). Criteria for unconscious cognition: three types of dissociation. Perception \& Psychophysics, 68(3), 489-504.

Shepard, R.N., \& Zare, S.L. (1983). Path-guided apparent motion, Science, 220, 632-634.

Smith, E. E. (1968). Choice reaction time: An analysis of the major theoretical positions. Psychological Bulletin, 69, 77-110.

Sohrabi, A. \& West, R.L. (2009). Positive and negative congruency effects in masked priming: A neuro-computational model based on representation, attention and conflict. Brain Research, 1289, 124-132.

Sternberg, S. (1969). The discovery of processing stages: Extensions of Donders' method. Acta Psychologica, 30, 276-315.

Sumner, P. (2007). Negative and positive masked priming - implications for motor inhibition. Advances in Cognitive Psychology, 3, 317-326.

Sumner, P. (2008). Mask-induced priming and the negative compatibility effect. Experimental Psychology, 55(2), 133-141.

Sumner, P., \& Brandwood, T. (2008). Oscillations in motor priming: positive rebound follows the inhibitory phase in the masked prime paradigm. Journal of Motor Behaviour, 40(6), 484-489.

Sumner, P., Nachev, P., Morris, P., Peters, A.M., Jackson, S.R., Kennard, C., \& Husain, M. (2007). Human medial frontal cortex mediates unconscious inhibition of voluntary action. Neuron, 54(5), 697-711.

Sumner, P., Tsai, P.-C., Yu, K., \& Nachev, P. (2006). Attentional modulation of sensorimotor processes in the absence of perceptual awareness. Proceedings of the National Academy of Sciences USA, 103 (27), 10520-25. 
Swick, D., Ashley, V., \& Turken, U. (2011). Are the neural correlates of stopping and not going identical? Quantitative meta-analysis of two response inhibition tasks, Neuroimage, $56,1655-1665$.

Robbins, R., \& McKone, E. (2003). Can holistic processing be learned for inverted faces? Cognition, 88, 79-107.

Tipples, J. (2008). Orienting to counterpredictive gaze and arrow cues. Perception and Psychophysics, 70 (1), 77-87.

Theeuwes, J., Kramer, A.F., Hahn, S., Irwin, D.E. (1998). Our eyes do not always go where we want them to go: capture of eyes by new objects. Psychological Science, 9, 379-385.

Tucker, M., \& Ellis, R. (1998). On the relations between seen objects and components of potential actions. Journal of Experimental Psychology: Human Perception and Performance, 24, 830-846.

Tucker, M., \& Ellis, R. (2004). Action priming by briefly presented objects. Acta Psychologica, 116, 185-203.

Vorberg, D., Mattler, U., Heinecke, A., Schmidt, T., \& Schwarzbach, J. (2003). Different time courses for visual perception and action priming. Proceedings of the National Academy of Sciences USA, 100, 6275-6280.

Ulrich, R., \& Miller, J. (2008). Response grouping in the psychological refractory period (PRP) paradigm: Models and contamination effects. Cognitive Psychology, 57, 75-121.

Van den Bussche, E., Van den Noortgate, W., \& Reynvoet, B. (2009). Mechanisms of masked priming: A meta-analysis. Psychological Bulletin, 135, 452-477.

Verleger, R., Görgen, S., \& Jaśkowski, P. (2005). An ERP indicator of processing of relevant gestalts in masked priming. Psychophysiology, 42, 677-690.

Verleger, R., Jaśkowski, P., Aydemir, A., van der Lubbe, R.H.J. \& Groen, M. (2004).Qualitative differences between conscious and non-conscious processing? On inverse priming induced by masked arrows. Journal of Experimental Psychology: General, 133, 494-515. 
Verleger, R., Vollmer, C., Waschkuhn, B., van der Lubbe, R.H.J., \& Wascher, E. (2000). Dimensional overlap between arrows as cueing stimuli and responses? Evidence from contra-ipsilateral differences in EEG-potentials. Cognitive Brain research, 10, 99-109.

Virzi, R. A., \& Egeth, H. E. (1985). Toward a translational model of Stroop interference. Memory and Cognition, 13, 304-319.

Weisskrantz, L., Warrington, E.K., Sanders, M.D., \& Marshall, J. (1974). Visual capacity in the hemianopic field following a restricted occipital ablation. Brain, 97, 709-728.

Wilms, M., Eickhoff, S.B., Specht, K. ,Amunts, K., Shah, N.J., Malikovic, A., \& Fink, G.R. (2005). Human V5/MT+: comparison of functional and cytoarchitectonic data. Anatomy and Embryology, 210(5-6), 485-495.

Wilson, A.D., Tresilian, J.R., \& Schlaghecken, F. (2010). Continuous priming effects on discrete response choices. Brain and Cognition, 74, 152-159.

Zorzi, M., and Umiltà, C. (1995). A computational model of the Simon effect. Psychological Research, 58, 193-205. 


\section{Appendix I}

Krüger, D. Klapötke, S., \& Mattler, U. (2011). PRP-paradigm provides Evidence for a perceptual locus of the negative compatibility effect. Consciousness \& Cognition, 20, 866881. doi:10.1016/j.concog.2010.09.014 


\section{Appendix II}

Krüger, D., \& Mattler, U. (in prep.). How features of the mask modulate inverse priming effects of unconscious visual stimuli. 


\title{
How features of the mask modulate inverse priming effects of unconscious visual stimuli
}

\author{
Daniel Krüger, \& Uwe Mattler
}

Georg-Elias-Müller Institute for Psychology, Georg-August University Göttingen, Germany

Send correspondence to:

Uwe Mattler

Georg-Elias-Müller Institute for Psychology

Georg-August University Göttingen

Gosslerstr. 14

D-37073 Göttingen

Germany

Email: uwe.mattler@psych.uni-goettingen.de 


\begin{abstract}
Inverse priming refers to the counterintuitive phenomenon of better classification performance of a target stimulus if the preceding prime belonged to an alternative response category. The magnitude of this effect is modulated by the structure of a masking stimulus being presented after the prime and prior to the target: If it contains features of the target stimuli (relevant masks) the effect is larger than if composed of only irrelevant features (irrelevant masks). However, the crucial features of relevant masks are unclear. Here we varied the structure of the mask in four levels by successively assimilating it to the target stimuli. Instead of a gradual increase of the effect, we found the same effect for three variants of irrelevant masks and increased effects with a clearly relevant mask, which consisted of a superimposition of the two target alternatives. With such a relevant mask, the size of inverse priming effects was the same irrespective of prime visibility. With all other masks, however, inverse priming was more pronounced within those subjects being less able to perceive the prime. These findings suggest two independent mechanisms which generate inverse priming effects: one mechanism operates independent of prime visibility with relevant masks, whereas the other mechanism operates only when the effective stimuli remain unconscious.
\end{abstract}




\section{Introduction}

A prime stimulus even if backward masked can influence classification of a subsequent target stimulus (Mattler, 2003; Vorberg, Mattler, Schmidt, Heineke, \& Schwarzbach, 2003). Performance benefits are observed when prime and target belong to the same category (congruent condition) as opposed to belonging to alternative categories (incongruent condition). Apart from these so-called positive priming effects, a counterintuitive reversal of priming effects with better performance in the incongruent condition has been reported in studies employing an additional masking stimulus which follows the prime and precedes the target (e.g. Eimer \& Schlaghecken, 1998). This phenomenon has been termed the negative compatibility effect (NCE) or simply inverse priming. Later on, several studies demonstrated that the size of this effect depended on the structure of the masking stimulus (e.g. Lleras \& Enns, 2004; Jaśkowski, \& Przekoracka-Krawczyk, 2005; Schlaghecken, \& Eimer, 2006; Verleger, Jaśkowski, Aydemir, van der Lubbe, \& Groen, 2004). In the literature, relevant masks which contain task-relevant features are distinguished from irrelevant masks which are entirely free of target-like elements. Most studies employed left- versus rightward pointing double arrows as prime and target stimuli composed of oblique lines only. With such stimuli a dense pattern of horizontally and vertically aligned lines could serve as an irrelevant mask. In contrast, a relevant mask could be built by simply superposing the two target alternatives. Typically, inverse priming effects with relevant masks are much larger as compared to irrelevant masks (Jaśkowski, 2007, 2008; Jaśkowski, \& Przekoracka-Krawczyk, 2005; Jaśkowski, Biłuńska, Tomanek, \& Verleger, 2008; Kiesel, Berner, \& Kunde, 2008; Lleras \& Enns, 2004, 2005, 2006; Schlaghecken, \& Eimer, 2006; Verleger et al., 2004, Verleger, Görgen, \& Jaśkowski, 2005). The Object Updating account of inverse priming (Lleras, \& Enns, 2004) provided an explanation for this difference. The main idea is that novel elements of the mask, i.e. all those elements not contained in the prime, possess a saliency advantage which is due to an iterative Updating process of visual input emphasizing novel elements of a dynamically changing input. According to the account, this saliency advantage of the primeopposite stimulus alternative results in faster processing of prime-incognruent targets which in turn leads to inverse priming. For obvious reasons, however, Object Updating does not explain inverse priming with irrelevant masks (Eimer \& Schlaghecken, 2002; Klapp, 2005; Klapp \& Haas, 2005; Lleras \& Enns, 2005; Schlaghecken, \& Eimer, 2006; Schlaghecken, Rowley, Sembi, Simmons, \& Whitcomb, 2007; Sumner, 2008). An alternative account of inverse priming, originally proposed by Eimer and Schlaghecken (2002) assumes that primeinduced activity is inhibited as a result of mask presentation. While Eimer and Schlaghecken 
(2002) emphasized that inhibition is self-triggered when the perceptual support for the prime is absent, others consider it as a process elicited by the mask irrespective of masking (Jaśkowski, 2007; Lleras, \& Enns, 2006; Mattler, 2005). Such an inhibitory process whether self-triggered or mask-triggered could also give the unprimed response alternative a relative advantage which might result in inverse priming. In contrast to the aforementioned ObjectUpdating account, these inhibitory accounts would readily explain inverse priming with both types of masks, relevant as well as irrelevant masks.

Apart from the question which mechanism(s) produce inverse priming for relevant and irrelevant masks another unresolved issue is which features constitute a relevant mask. A relevant mask is characterized by the fact that it contains both target alternatives. The most typical example is a mask formed by overlaying the two target alternatives. Presentation of such a mask is equal to the onset of the alternative prime (Verleger, et al., 2004). However, several studies employed masks composed of random lines of differing orientations (similar to the diagonal mask used in the current study, cf. Figure 1). While it seems clear that the presentation of such a mask is not equal to the appearance of the prime-opposite stimulus, one might argue that all arrow-like elements within such a mask possessing the prime-opposite orientation become particularly salient due to Object Updating giving rise to inverse priming. Whether such random line masks (RLMs) built by oblique lines have to be considered as relevant masks or irrelevant masks remains an open question. Empirical findings are divergent. On the one hand, Schlaghecken and Eimer (2006) found inverse priming effects about twice as large for RLMs of diagonal lines as opposed to RLMs of horizontally and vertically lines only (which were thus irrelevant masks) suggesting that the diagonal mask should be considered a relevant mask. On the other hand, however, Sumner (2008) reported inverse priming effects whose size did not differ for RLMs either containing or sparing out target orientations (targets were horizontal and vertical line pairs). The current study is intended to isolate the crucial feature of a relevant mask by comparing inverse priming effects across four different masks whose structure is successively assimilated to the target stimuli.

\section{Methods}

Design Rationale. A relevant mask is characterized by larger inverse priming effects compared to a completely irrelevant mask which therefore serves as a reference in our study. This reference mask was composed of a pattern of horizontally and vertically oriented lines 
(the orthogonal mask; all mask types are depicted in Figure 1). We used double arrows as primes and targets and thus such a mask is completely neutral and free of target-like features. We compared the size of inverse priming effects for three further masks to this reference mask. Apart from the superposition mask which should clearly lead to larger effects as reported in the literature (e.g. Lleras, \& Enns, 2004), we created two pattern masks. One was built by intersecting oblique lines (diagonal mask, comparable to the mask used by Schlaghecken, \& Eimer, 2006) which could thus randomly entail (at least some) task-relevant arrow-like elements. Another mask was composed of several arrows equal to those forming primes and targets which is termed arrow pattern mask. Thus, target features are clearly present in this mask.

Thus, we employed a continuum of three masks whose features were more and more similar to the target elements. We expected a discontinuity in the increase of inverse priming effects from the reference mask to the superposition mask. If inverse priming effects were larger only with the superposition mask but indistinguishable with the other masks, results would suggest that inverse priming induced by Object Updating requires that masks are built by both prime alternatives. If, in contrast, the presence of (exactly the) target elements in the mask is crucial, larger inverse priming would already be expected for the arrow pattern mask while effects are identical for the diagonal and orthogonal mask. And if, on the other hand, the mere presence of target-similar features as randomly contained in the diagonal mask would suffice to trigger inverse priming via Object Updating the diagonal mask should yield larger inverse priming effects compared to the reference mask. The first increase in inverse priming effects with respect to the reference mask would suggest that task-relevant features contained in this mask are extractable by the visual system and therefore facilitate processing of an incongruent target.

Participants. 54 participants took part in the current experiment. Each group consisted of 12 participants while 18 participants were in the orthogonal mask condition (26 male, mean age 23.5 years, std 2.7 years, range 19-33 years). 50 were right-handed by self-report, and all had normal or corrected-to-normal vision. Each participant received $7 €$ per hour for participation, adding up to $24.50 €$ for the three sessions in the experiment.

Tasks. Participants performed three sessions with two different tasks. (a) Choice-RT task: In the first and second session participants had to judge the orientation of a double arrow pointing either left- or rightwards $(<<,>>$, see Figure 1) by pressing a corresponding button with the left or right index finger. (b) Prime-recognition: In the third session participants were 
informed about the presence of the primes and they should indicate the prime orientation in the same way.

Stimuli. Primes and target stimuli were identical double arrows which possessed the same orientation on half of the trials (congruent trials) and which were of opposite orientations on the remaining trials (incongruent trials). Except for the superposition mask, a new mask was presented on each trial to avoid perceptual learning which might aid in prime recognition (e.g. Schlaghecken, Blagrove, \& Maylor, 2008). The orthogonal masks were composed of 130 line elements of varying length and width (within limits) which were centred on a $13 \times 10$ grid (including a spatial jitter). In contrast, the diagonal masks were composed of only 30 lines centred on a 6x5 gird. Line width was constant and identical to prime and target stimuli. The arrow pattern mask comprised 15 arrows identical to the target stimuli which were placed on every second point of the 6x5 grid. In each arrow pattern mask, the frequency of the leftward and rightward oriented arrows was matched. All stimuli were presented in black on white background at the centre of the monitor. Primes, target stimuli and superposition masks subtended approximately $1^{\circ} \times 1.5^{\circ}$ of visual angle. The grid points of the other masks subtended approximately $1.3^{\circ} \times 1.7^{\circ}$ (maximum area of the mask $2.8^{\circ} \times 2.2^{\circ}$ ). During all sessions, incorrect responses were signalled by visual error feedback (translated from German: "Error").

Please insert Figure 1 about here

Procedure. Participants were tested individually on separate days. In a dimly lit room they sat $100 \mathrm{~cm}$ in front of a computer monitor with the chin fixed in a rest. The initial and the prime recognition session started with instructions and a practice section. In the first session, participants were familiarized with the target stimuli and performed 48 demo-trials which were identical to those of the experiment. At the beginning of the third session, participants were told about the presence of prime stimuli and they were shown the entire stimulus sequence with long stimulus durations to ensure that they understood the prime recognition task. Participants were instructed to identify prime stimuli as accurately as possible without speed stress. Apart from this initial part, the third session was identical to the first two sessions. 
The stimulus sequence is depicted in Figure 1. Stimuli and timing parameters were identical across all three sessions. A central fixation cross that was presented for $500 \mathrm{~ms}$ indicated the start of each trial. After a blank interval of $300 \mathrm{~ms}$ the prime was shown for $20 \mathrm{~ms}$. After a prime-mask SOA of $30 \mathrm{~ms}$ the mask was presented for $100 \mathrm{~ms}$ which in turn was followed by the target, again presented for $100 \mathrm{~ms}$. The mask-target SOA varied between 0, 100, 150, 200 ms. The 0-ms-SOA served as a control condition by omitting the mask. For this reason, positive priming was expected to occur. In the first two sessions, the computer monitored for a response for $1800 \mathrm{~ms}$ while during prime recognition response latencies were unlimited. After response delivery the next trial started after a variable inter-trial-interval (range 1250$2050 \mathrm{~ms}$, mean ca. $1115 \mathrm{~ms}$, pseudo-exponentially distributed).

Apparatus. The experiment was run on a personal computer with a CRT-display $(100 \mathrm{~Hz}$ vertical refresh rate, resolution 1024 x 768). During all three sessions, response buttons were the left and right CTRL-buttons of the computer keyboard.

Design. In the choice RT task of the first two sessions, a three-factorial design with two dependent variables (response times and error rates) was implemented. Mask-type varied between subjects (orthogonal, diagonal, arrow pattern, superposition mask), SOA (0, 100, 150, $200 \mathrm{~ms}$ ) and Congruency (congruent vs. incongruent) were within-subject factors. Prime recognition performance was summarized by d' for each mask-type and SOA separately. Arbitrarily, leftward-oriented arrows were defined as signal and d' was derived for each target separately and then averaged. 16 Blocks of 48 trials were acquired in each session yielding 96 replications for each of the 8 experimental conditions resulting from the factorial combination of SOA and Congruency. Prime visibility estimates were based on 192 trials per SOA and mask-type.

Within each session the order of trials was pseudo-randomized. However, we initially suspected that the order of presenting the different mask-target SOAs (especially the temporal position of the $0-\mathrm{ms}-\mathrm{SOA}$ ) to the subject would matter. We systematically investigated a potential influence of SOA presentation order by presenting SOAs either intermixed or blocked (0-ms-SOA first versus at the end of the experiment) for those 18 subjects in the diagonal mask condition. Six participants performed the 0-ms-SOA condition first, another six participants performed it last and the remaining six participants received a randomized presentation of SOA conditions (just like all the other 36 subjects of the other mask-type groups). Within the two blocked-presentation groups, the positive SOA conditions were also blocked and their order was counter-balanced across subjects. We subjected these data to a 
mixed ANOVA with between-subjects factors SOA-presentation order and within-subjects factors SOA, Congruency and Session (first vs. second). However, priming effects on RTs and error rates turned out to be independent of SOA presentation order (RT and ER: interaction presentation order x Congruency $\mathrm{F}(6,45)<1$ ), even when including SOA (RT and ER: $\mathrm{F}(6,45)<1)$ or Session (RT and ER: $\mathrm{F}(2,15)<1)$ or both $(\mathrm{RT}: \mathrm{F}(6,45)<1$, ER: F(6,45) = $1.78, \mathrm{p}=.124$ ) as additional factors in the model. Thus, we did not find any evidence for an influence of SOA presentation order on inverse priming effects and thus we collapsed the data for all further analyses across the three groups within the diagonal mask group.

Statistical Analysis. RTs in the choice RT task were summarized by trimmed means: the two fastest and three slowest responses were discarded for each participant and condition. Error and post-error trials were also excluded. RTs and arc-sine transformed error rates were analyzed separately by means of a mixed three-way ANOVA. We carried out two separate ANOVAs for each dependent measure: one including all levels of SOA, one including only those levels of SOA supposed to lead to inverse priming (100, 150, $200 \mathrm{~ms})$. To isolate the crucial mask feature making up a relevant mask we intended to compute a set of orthogonal a priori contrasts if the ANOVA restricted to those SOAs where inverse priming is expected to occur $(100,150,200 \mathrm{~ms})$ indicates a modulation of the size of inverse priming effects by mask structure. To this end, priming effects for each mask-type are contrasted to the baseline condition built by the irrelevant orthogonal mask. If the interaction of mask-type and Congruency is also modulated by SOA such an analysis should be done for each SOA separately.

\section{Results}

$R T$. Results are depicted in Figure 2. Mask-type influenced response times, $\mathrm{F}(3,50)=5.7$, $\mathrm{p}<$ .01. Increasing mask-target similarity, lead to slower responses $(380,371,406,414 \mathrm{~ms}$ for the orthogonal, diagonal, arrow pattern and superposition mask, respectively), probably due to poorer target salience. When a mask was presented, larger mask-target SOAs lead to faster responses (413, 387, $373 \mathrm{~ms}$ for the 100, 150, $200 \mathrm{~ms}$ SOA). When the mask was omitted (0ms-SOA condition), response speed was intermediate $(388 \mathrm{~ms})$. The influence of SOA is reflected in the significant main effect of SOA, $\mathrm{F}(3,150)=115.6, \mathrm{p}<.00001$. Furthermore, also the interaction of mask-type and SOA reached significance, $F(9,150)=2.75 ; p<.01-$ RT performance benefited over-additively from increasing SOA and by employing target- 
dissimilar masks. Considering priming effects, when all levels of SOA were included in the analysis, a main effect of Congruency was absent, $\mathrm{F}(3,50)<1$. As expected, the 0 -ms-SOA led to positive priming effects $(+111 \mathrm{~ms})$ while at all other SOAs inverse priming was observed as reflected by the significant interaction of Congruency and SOA, F(3,150) = $421.1 ; \mathrm{p}<.00001$. When analysing positive SOAs $(100,150,200)$ alone, the main effect of Congruency was significant, $\mathrm{F}(1,50)=165.0 ; \mathrm{p}<.00001$. However, the magnitude of inverse priming was still influenced by SOA, $\mathrm{F}(2,100)=24.2 ; \mathrm{p}<.00001$ : inverse priming decreased with increasing SOA (-42 ms, $-37 \mathrm{~ms},-27 \mathrm{~ms}$, respectively). Most importantly for the purpose of the current study, inverse priming was also affected by mask-type which became manifest in the three-way interaction of mask-type, SOA and Congruency for the 100,150, $200 \mathrm{~ms}$ SOA, $\mathrm{F}(6,100)<3.67, \mathrm{p}<.01$. Thus, the time-courses of inverse priming effects differed across the mask types. Inverse priming effects were largest for the superposition mask, especially at the 100 and $200 \mathrm{~ms} \mathrm{SOA}$ (see Figure 2B).

To disentangle which mask included the crucial feature inducing inverse priming via Object Updating we compared priming effects for each mask to the orthogonal mask separately at each SOA using t-contrasts. Only the superposition mask produced larger inverse priming effects relative to the reference condition at least in the 100 and 200-ms-SOA condition: at the $100 \mathrm{~ms} \mathrm{SOA}$ the effects were $35 \mathrm{~ms}$ larger $(\mathrm{t}(22)=7.8, \mathrm{p}<.0001)$, at the $150 \mathrm{~ms}$ SOA $7 \mathrm{~ms}$ $(\mathrm{t}(22)=1.5 ; \mathrm{p}=.15)$ and at the $200 \mathrm{~ms}$ SOA $18 \mathrm{~ms}(\mathrm{t}(22)=4.0, \mathrm{p}<.001)$. All other comparisons between mask-types for the three SOAs did not reach significance, with two exceptions: inverse priming effects for the diagonal mask were significantly smaller than for the reference mask at the $150 \mathrm{~ms} \mathrm{SOA}(9 \mathrm{~ms}, \mathrm{t}(28)=2.3 ; \mathrm{p}<.05)$ and at the $200 \mathrm{~ms}$ SOA $(9$ $\mathrm{ms}, \mathrm{t}(28)=2.2 ; \mathrm{p}<.05)$.

Please insert Figure 2 about here

Accuracy data. Results are depicted in Figure 2. Errors occurred on $3.6 \%$ of all trials. Error rates differed between mask types, $\mathrm{F}(3,50)=6.2 ; \mathrm{p}<.01$ : error rates were highest for the diagonal mask $(4.9 \%)$ and the arrow pattern mask (3.9\%) and lower for the orthogonal (2.4 $\%)$ and the superposition mask $(1.8 \%)$. Error rates were also influenced by SOA, F(3,150) = 14.6; $\mathrm{p}<.00001$ : when a masking stimulus was presented error rates increased with SOA $(2.6 \%, 2.7 \%, 4.5 \%)$ and were intermediate at the 0 -ms-SOA condition $(4.1 \%)$. Furthermore 
and interaction of both factors was observed, $\mathrm{F}(9,150)=5.0 ; \mathrm{p}<.00001$. Across all SOAs, no evidence for priming effects was obtained, $F(1,50)<1$. In contrast, the significant interaction of Congruency and SOA, F(3,150) = 142.8; $\mathrm{p}<.00001$, is due to positive priming at the 0 -msSOA condition $(+7.4 \%)$ and inverse priming at the other SOA conditions $(-2.3 \%,-2.1 \%$, $3.2 \%$ ). When positive SOA conditions were analyzed alone the interaction of Congruency and SOA was not significant, $\mathrm{F}(2,100)=1.7 ; \mathrm{p}=.18$, but the time-courses of inverse priming effects differed according to mask-type as indexed by the three-way interaction of Congruency, SOA and mask-type, $\mathrm{F}(6,100)=2.7 ; \mathrm{p}<.02$.

When comparing inverse priming for each mask to the reference mask (for each SOA) inverse priming was significantly larger for the superposition mask at the $100 \mathrm{~ms}-\mathrm{SOA}, \mathrm{t}(22)=3.3 ; \mathrm{p}$ $<.005$. Additionally, we found significantly smaller inverse effects for the diagonal mask at the 100-ms-SOA compared to the reference mask, $\mathrm{t}(28)=2.5 ; \mathrm{p}<.03$. For all other masks and SOAs, inverse priming effects were indistinguishable from the baseline condition.

Prime visibility. As expected, prime visibility depended on the presence of a masking stimulus as indexed by the significant main effect of SOA, $F(3,150)=619.6 ; p<.0001$. While participants could perceive the prime almost perfectly when no mask was present $\left(\mathrm{d}^{\prime}=2.03\right)$, discrimination performance was poor at the other SOA conditions $\left(\mathrm{d}^{\prime}=.17, .10, .09\right.$, respectively) and did not differ significantly across these conditions, $\mathrm{F}(2,100)=2.5, \mathrm{p}=.09$. Similarly, mask-type did not affect prime recognition performance, $F(3,50)=1.1, p=.36$, but masking functions differed between the mask-types as indexed by the interaction of SOA and mask type, $\mathrm{F}(9,150)=2.1, \mathrm{p}<.03$. However, this difference was due to the unmasked SOA condition because when excluding this condition, the interaction was far from being significant, $\mathrm{F}(6,100)<1$. Interestingly, prime recognition performance seemed to be poorer in the 0 -ms-SOA condition for the superposition masks ( $d^{\prime}=1.7$ as opposed to $d^{\prime}=2.1,2.2,2.1$ for the three other masks) even though these conditions were physically identical and only differed with respect to the mask employed on the other trials. Disregarding the 0-ms-SOA condition, subject's discrimination performance did not significantly differ from chance level for any SOA nor mask-type ( $\mathrm{d}^{\prime}<.23, \mathrm{t}<2.0, \mathrm{p}>.06$ ). Nevertheless, prime recognition performance varied substantially across participants (min d' $=-.77$, $\max \mathrm{d}^{\prime}=1.58$ ) which led us to test whether inverse priming was affected by prime visibility.

Prime visibility and inverse priming. In a first attempt, we calculated Pearson correlation coefficients for priming effects and prime visibility separately for each mask-type and SOA. In the 0 -ms-SOA condition, priming effects were independent of prime visibility $(r=-.44$, 
$.16, .38, .23, \mathrm{p}>.14$ for the orthogonal, diagonal, arrow pattern and superposition mask, respectively). In contrast, the magnitude of inverse priming seemed to differ according to the individual ability to perceive the prime for the orthogonal, diagonal and arrow pattern mask for the other SOAs (averaged across SOA 100, 150 and 200: $\mathrm{r}=.56, .66, .49, \mathrm{p}<.09, .02, .30$, respectively). However, using the superposition mask such a relationship was clearly absent ( $\mathrm{r}$ $=-.02, \mathrm{p}>.43$ ). Figure 3 illustrates the dependency of RT-priming effects on prime visibility. Visual inspection suggests that a linear model is not the best way to describe the relationship among prime visibility and priming effects. At least for the orthogonal and diagonal mask, it seems more adequate to distinguish between two groups of participants according to prime visibility. The vast majority of participants seemed to be unable to perceive the prime and reliably shows inverse priming. A minority which showed above-chance level performance in prime recognition tended towards positive priming effects.

Please insert Figure 3 about here

To further test the hypothesis that chance level performance in the prime recognition task (subliminal prime perception) was accompanied by inverse priming effects and above-chance level performance (supraliminal prime perception) by positive priming we had to introduce a numerical criterion to distinguish sub- from supra-threshold prime perception. A determination of such a threshold for conscious prime perception is necessarily arbitrary. Taking into account the unequal numbers of participants showing good as opposed to poor prime identification performance we subjected prime visibility data (from 100, 150, $200 \mathrm{~ms}$ SOA) to a kmeans-cluster analysis for $\mathrm{k}=2$ groups. The cluster analysis generated a cluster of four participants who demonstrated above-chance level prime perception (average $\mathrm{d}^{\prime}=1.3$, 1.2, 1.2 for the respective SOAs, $\mathrm{t}(3)>5.8, \mathrm{p}<.02)$, while the performance of the remaining 50 participants did not differ significantly from chance level $\left(d^{\prime}=.07, .02, .01 ; \mathrm{t}(49)<2.3 ; \mathrm{p}\right.$ $>.06$, one-tailed). After having established these two groups showing sub- vs. supra-liminal prime perception, we analyzed whether both groups also differed with respect to priming effects. Therefore, we included Visibility Group (sub- vs. supraliminal) as an additional factor in a mixed ANOVA model (with SOA 100, 150, $200 \mathrm{~ms}$ and Congruency as further factors). Priming effects differed between the two groups as indexed by the interaction of Congruency and Visibility Group with respect to both, RTs: $\mathrm{F}(1,48)=9.7 ; \mathrm{p}<.01$, as well as error rate 
data, $\mathrm{F}(1,48)=12.5 ; \mathrm{p}<.001$. Inverse priming was found among those subjects showing subliminal prime perception $(-38 \mathrm{~ms}, \mathrm{t}(49)=11.8, \mathrm{p}<.0001$; and $-3.1 \%, \mathrm{t}(49)=4.7, \mathrm{p}<$ .0001) but priming effects were absent among those subjects showing supraliminal prime perception $(-2 \mathrm{~ms}, \mathrm{t}(3)<1$; and $+3.8 \%, \mathrm{t}(3)=1.1, \mathrm{p}>.05)$.

Taken together, across all masks the cluster-analytical approach revealed a qualitative difference with respect to priming effects: only those subjects being unable to perceive the primes showed subliminal priming. However, the role of prime visibility might crucially depend on whether a relevant or irrelevant mask is used as proposed by Klapp (2005). While, according to his view, inverse priming effects grow with increasing prime visibility for relevant masks, effects grow with deteriorating prime visibility for irrelevant masks. To assess this hypothesis, we had to explore the relationship of prime visibility and priming effects for each mask separately. However, correlations of prime visibility and prime recognition might be largely driven by the data from few subjects showing supraliminal prime perception for the orthogonal and diagonal mask (cf. Figure 3). To achieve comparable conditions for all masks, we excluded these four subjects and calculated correlations only among the remaining 50 subjects showing subliminal prime perception for each mask.

We found substantial positive correlations for each SOA and mask-type apart from the superposition mask (orthogonal: .52, .38, .41; diagonal: .09, .62, .61; arrow pattern: .37, .33, .75 ; superposition: $-.25, .06, .10)$. The mean correlation averaged across SOAs for the orthogonal, diagonal and the arrow pattern mask was .45. Due to the small sample sizes (11, $15,12,12$ subjects for the orthogonal, diagonal, arrow pattern and superposition mask, repectively), however, correlations only reached significance for the 150-ms-SOA for the diagonal mask and the 200-ms-SOA for the diagonal and the arrow pattern mask (each $\mathrm{p}<$ .02). In order to assess whether positive relationships between priming and prime visibility for all but the superposition mask truly exist we performed a permutation-test. The aim is to estimate the probability for the observed mean correlation of .45 to result from completely uncorrelated data. To this end, we generated 10,000 times 12 random number pairs (each of the nine correlations reported above is on average also based on data from 12 subjects) from a standardized-normal distribution and calculated the correlation for each set of 12 pairs. Then, we randomly sampled 10,000 times sets of 9 correlations (representing those correlations for the orthogonal, diagonal and arrow pattern mask) from this pool and calculated the mean correlation to derive its distribution. The one-tailed p-value can be determined by estimating the proportion of values from this distribution being larger than the observed mean 
correlation. However, all of the values were smaller than $r=.45$ thus resulting in $p=0$. To get a better estimate, we repeated the procedure 10 times and only considered the maximum value of the distribution. On 9 out of 10 iterations, the maximum mean correlation was smaller than the observed one of .454 (mean of maximums .37) and only in one case marginally larger (.460) indicating that the p-value for the rejection of the null hypothesis that the positive correlations between prime visibility and priming effects occurred by chance is actually almost indistinguishable from zero.

For the superposition mask, we observed correlations close to zero for each SOA. The observed mean correlation of -.02 is representative for the distribution of mean correlations among triplets of correlations from uncorrelated random variables (.43-quantile, mean $r=0)$. The same is true for the maximum correlation within the observed triplet (.10) which corresponds to the .26-quantile of the distribution of maxima from triplets of random correlations (mean max $\mathrm{r}=.25$ ). Additionally, we calculated the probability that the correlation triplet for the superposition mask stems from the same distribution of correlations as the other three masks. To this end, we pooled all 12 correlation coefficients and generated the common distribution of mean correlations among all possible triplets (220 possibilities to choose 3 out of 12 values). Strikingly, such a small or a smaller mean correlation only arises on only 2 out of 220 cases which means that the null hypothesis that the three correlations stem from the same population as the other nine correlations can be rejected at $\mathrm{p}=.009$.

Thus, even when controlling for the different absolute level of prime recognition performance between the masks by excluding those subjects with supraliminal prime perception, we still found a linear relationship between priming effects and prime visibility for the orthogonal, diagonal and arrow pattern mask but not for the superposition mask. Except for this mask, correlations were positive indicating that inverse priming effects were larger for those subjects who performed especially poor in prime discrimination.

\section{Discussion}

We compared inverse priming effects between four mask-types which differ with respect to the similarity of their features to the target stimuli. We intended to isolate the crucial feature of a relevant mask which allows for larger inverse priming effects due to Object Updating as compared to the irrelevant reference mask. We found that only priming effects for the superposition mask were larger while the size of the effect was indistinguishable across the 
orthogonal, the diagonal and the arrow pattern mask. Additionally, we found that the relationship of prime visibility and priming effects differed between the superposition mask and the other masks employed. These results add further support to the notion that random line masks which also contain target-like features do not trigger Object Updating leading to larger inverse priming effects as demonstrated in another recent study (Sumner, 2008). Therefore, the mere presence of target features as part of the mask does not seem to be sufficient to induce larger inverse priming effects. At least with respect to the RLMs adopted in this study, the visual system seems incapable of extracting target-like elements from the mask. What is then the essential difference with the superposition mask? In contrast to RLMs, presentation of the superposition mask creates the perception of adding a second (opposite) prime when the mask appears on the screen (Verleger, et al., 2004). Such a perception seems to be absent in the arrow pattern mask. Therefore, the explanatory power of Object Updating for inverse priming seems to be limited to such superposition masks when the onset of a "second prime" is clearly identifiable. The Object Updating account claimed that in general successively shown stimuli are integrated into a common underlying representation (Lleras, \& Enns, 2004). However, in the case of inverse priming prime and mask might only be held for different instantiations of a single object if they are sufficiently similar. If the mask differs with respect to size, number of elements or position of the elements (like RLMs) it does not seem to be integrated into the existing prime representation but instead considered an independent object whose exact (rather complex) structure is considered as being unrelated to the current task. Further evidence for the view that the critical feature of a relevant mask is that the target features have to be easily extractable from the mask is provided by another recent study. Jaśkowski (2009) compared two masks being composed of the two target alternatives (left- and right-pointing triangles, 4 and $>$ ) which were either superimposed (resulting in a star-shaped object) or positioned side-by-side (resulting in a double-arrow). According to Object Updating, both masks should therefore lead to the same amount of inverse priming. But larger inverse priming effects were found for the double-arrow mask which made extraction of the target-elements much easier. When the mask is shown the perception of the prime stimulus "jumping" to a new position is generated while simultaneously the prime-opposite stimulus also appears which yields faster processing of incongruent targets.

We were not the first using an arrow pattern mask in the context of inverse priming. Contrary to our findings, Przekoracka-Krawczyk and Jaśkowski (2007) demonstrated that inverse priming effects grew with training when an arrow pattern mask was used and that the absolute 
size depended on the complexity of this mask (i.e. number of elements). In a prior study, Klapp and Hinkley (2002) demonstrated in the same way dependency of inverse priming on training with potentially relevant letter string masks. Przekoracka-Krawczyk and Jaśkowski (2007) argue that perceptual learning of mask structure causes the increase of inverse priming effects during the experiment because subjects learn to perceptually extract target-like features from the mask which potentiates inverse priming effects via perceptual interactions with the prime. In our study, an increase relative to the completely irrelevant mask should have been the consequence. But we failed to observe such an increase even when considering the second session alone. Why didn't we find larger effects for the arrow pattern mask? First, our mask contained more elements even than the more complex one of PrzekorackaKrawczyk and Jaśkowski (2007) potentially hampering the visual extraction of target-like features within the mask. Second, in the study of Przekoracka-Krawczyk and Jaśkowski (2007) participants always viewed the same mask whereas in our study trial-to-trial changes might have prevented perceptual learning of mask content. This argument is supported by findings from Schlaghecken, Blagrove and Maylor (2008) who demonstrated that prime recognition performance was clearly better when subjects viewed always the same mask compared to another group of subjects who were exposed to a different mask on each trial. If prime recognition is facilitated by presenting the same mask on each trial the extraction of target-like features might also be facilitated explaining the larger effects found by Przekoracka-Krawczyk and Jaśkowski (2007). Potentially conflicting with this interpretation, Schlaghecken et al. (2008) observed unaltered inverse priming effects after five sessions but they were using a RLM similar to our diagonal mask, not the arrow pattern mask. Therefore, keeping mask structure constant throughout the experiment, using a mask which contains exactly the target features and which is not too complex (Przekoracka-Krawczyk, \& Jaśkowski, 2007) might also allow for recognition of task-relevant features within the mask as a result of perceptual learning which in turn leads to preparation of the unprimed response.

To sum up, presentation of a relevant mask creates the perception of the onset of a second prime (of opposite direction) which seems to be limited to such masks that easily allow extracting target features from the mask display. Such a perception easily arises for superposition masks but can also be achieved via perceptual learning when the masks remains unchanged throughout the experiment and mask features resemble target stimuli. 


\section{Differential impact of prime visibility suggests two separate mechanisms}

Some participants possessed an exceptional capacity to perceive masked primes. As previously reported by Sumner (2007), within this minority of subjects, processing of prime and mask seems to differ from the other participants resulting in smaller or even positive priming effects which is an example for a qualitative processing difference between conscious and unconscious stimuli. It could be due to larger positive priming effects induced by visible primes that are only partially inhibited by the mask as proposed by Sumner (2007). However, the vast majority of participants were incapable of perceiving the prime and showed inverse priming within a typical range. Interestingly, within this group the magnitude of inverse priming still correlated with the individual prime recognition performance but only for those masks which turned out to be irrelevant providing evidence for the view that two separate mechanisms (with different characteristics) are at play for the two kinds of masks which could be due to Object Updating being such a dominant process for the superposition mask that it eliminates the influence of an inhibitory mechanism suppressing prime activation. This view receives further support from the fact that time-courses of priming effects also differed between the two kinds of masks.

Klapp (2005) argued for the view of two completely distinct phenomena with relevant and irrelevant masks which are differentially influenced by prime visibility. According to his view, for irrelevant masks a positive correlation occurs (which we also found) while Object Updating could be more effective for visible primes implying a negative correlation of priming effects and prime visibility (which we did not observe). At first glance, such a relationship for relevant masks seems to be at odds with our data. However, Klapp's (2005) argument is based on a review of previously published data (Lleras, \& Enns, 2004). Prime visibility and the magnitude of inverse priming varied in this study as a function of primemask SOA, but in contrast to our study prime visibility was generally much larger (worst prime recognition performance at their $15 \mathrm{~ms}$ prime-mask SOA of about $60 \%$, otherwise up to $90 \%$ correct). Therefore, it could be that such a negative correlation for relevant masks exists but that we did not capture it due to almost perfect masking in our study for every participant in this masking condition. Alternatively, the observation made by Klapp (2005) might be wrong because one has to keep in mind that comparing prime visibility and priming effects across conditions (within the same subjects) always implies the risk of analyzing a relationship that is actually the result of the manipulation of a third factor affecting both priming and visibility (and the prime-mask SOA is supposed to be a possible candidate for 
such a factor, cf. Also Schlaghecken, et al., 2008). Therefore, Object Updating is maybe not more effective with better visible primes but instead it is fostered by increasing prime-mask SOAs which also lead to better prime visibility. Future studies have to clarify whether prime visibility is also directly linked to priming effects with relevant masks. With irrelevant masks, converging evidence suggests that prime visibility is positively related to inverse priming (see also Eimer, \& Schlaghecken, 2002). Thus, with both types of masks, prime visibility seems to play a different role which could be interpreted as evidence for two completely independent mechanisms generating the effect with both masks as proposed by Klapp (2005). 


\section{References}

Eimer, M. \& Schlaghecken, F. (1998). Effects of masked stimuli on motor activation: Behavioral and electrophysiological evidence. Journal of Experimental Psychology: Human Perception and Performance, 24, 1737-1747.

Eimer, M., \& Schlaghecken, F. (2002). Links between conscious awareness and response inhibition: Evidence from masked priming. Psychonomic Bulletin \& Review, 9, 514-520.

Jaśkowski, P. (2007). The effect of nonmasking distractors on the priming of motor responses. Journal of Experimental Psychology: Human Perception and Performance, 33, $456-468$.

Jaśkowski, P. (2008). The negative compatibility effect with nonmasking flankers: A case for mask-triggered inhibition hypothesis. Consciousness and Cognition, 17, 765-777.

Jaśkowski, P. (2009). Negative compatibility effect: the object-updating hypothesis revisited. Experimental Brain Research, 193, 157-160.

Jaśkowski, P., Biłuńska, A., Tomanek, M., \& Verleger, R. (2008). Mask- and distractortriggerd inhibitory processes in the priming of motor responses: An EEG study. Psychophysiology, 45, 70-85.

Jaśkowski, P., \& Przekoracka-Krawczyk, A. (2005). On the role of mask structure in subliminal priming. Acta Neurobiologiae Experimentalis, 65, 409-417.

Klapp, S. T. (2005). Two versions of the negative compatibility effect: Comment on Lleras and Enns (2004). Journal of Experimental Psychology: General, 134, 431-435.

Klapp, S. T., \& Haas, B. W. (2005). Nonconscious influence of masked stimuli on response selection is limited to concrete stimulus-response associations. Journal of Experimental Psychology: Human Perception and Performance, 31, 93-209.

Klapp, S. T., \& Hinkley, L. B. (2002). The negative compatibility effect: Unconscious inhibition influences reaction time and response selection. Journal of Experimental Psychology: General, 131, 255-269.

Kiesel, A., Berner, M.P., \& Kunde, W. (2008). Negative congruency effects: A test of the inhibition account. Consciousness and Cognition, 17, 1-21. 
Lleras, A. \& Enns, J.T. (2004). Negative compatibility or object updating? A cautionary tale of mask-dependent priming. Journal of Experimental Psychology: General, 133, 475-493.

Lleras, A. \& Enns, J.T. (2005). Updating a cautionary tale of masked priming: Reply to Klapp (2005). Journal of Experimental Psychology: General, 134, 436-440.

Lleras, A., \& Enns, J. T. (2006). How much like a target can a mask be? Geometric, spatial, and temporal similarity in priming: A reply to Schlaghecken and Eimer (2006). Journal of Experimental Psychology: General, 135, 495-500.

Loftus, G. R., \& Masson, M. E. J. (1994). Using confidence intervals in within-subject designs. Psychonomic Bulletin \& Review, 1, 476-490.

Mattler, U. (2003). Priming of mental operations by masked stimuli. Perception and Pschophysics, 65, 167-187.

Mattler, U. (2005). Inhibition and decay of motor and non-motor priming. Perception and Pschophysics, 67, 285-300.

Przekoracka-Krawczyk, A., \& Jaśkowski, P. (2007). Perceptual learning can reverse subliminal priming effects. Perception \& Psychophysics, 69(4), 558-566.

Schlaghecken F., Blagrove E., \& Maylor, E.A. (2008). No difference between conscious and nonconscious visuomotor control: evidence from perceptual learning in the masked prime task. Consciousness and Cognition. 17(1), 84-93.

Schlaghecken, F., \& Eimer, M. (2006). Active masks and active inhibition: A comment on Lleras and Enns (2004) and on Verleger, Jaśkowski, Aydemir, van der Lubbe, and Groen (2004). Journal of Experimental Psychology. General, 135, 484-494.

Schlaghecken, F., Rowley, L., Sembi, S., Simmons, R., \& Whitcomb, D. (2007). The negative compatibility effect: A case for self-inhibition. Advances in Cognitive Psychology, 3, 227-240.

Sumner, P. (2007). Negative and positive masked priming - implications for motor inhibition. Advances in Cognitive Psychology, 3, 317-326.

Sumner, P. (2008). Mask-induced priming and the negative compatibility effect. Experimental Psychology, 55(2), 133-141. 
Verleger, R., Görgen, S., \& Jaśkowski, P. (2005). An ERP indicator of processing of relevant gestalts in masked priming. Psychophysiology, 42, 677-690.

Verleger, R., Jaśkowski, P., Aydemir, A., van der Lubbe, R.H.J. \& Groen, M. (2004).Qualitative differences between conscious and non-conscious processing? On inverse priming induced by masked arrows. Journal of Experimental Psychology: General, 133, 494-515.

Vorberg, D., Mattler, U., Heinecke, A., Schmidt, T., \& Schwarzbach, J. (2003). Different time courses for visual perception and action priming. Proceedings of the National Academy of Sciences USA, 100, 6275-6280. 


\section{Figure Legends}

Figure 1. Stimuli used in the experiment. Primes and targets were congruent on half of the trials and otherwise incongruent. Mask-target SOA varied randomly from one trial to the next. Four different masks were employed, each one presented to a different group of participants. The orthogonal mask served as a reference condition. Inverse priming effects for the other masks were compared to this reference. Larger inverse priming effects with one of the other masks would suggest that the corresponding mask contains the task-relevant feature triggering object updating.

Figure 2. A) Response time and error rate results for the orthogonal, the diagonal, the arrow pattern and the superposition mask are depicted. B) Net priming effects on RT data for the four masks. While positive priming occurred for the 0 -ms-SOA condition, inverse priming was found for the other SOAs. The magnitude and time course of inverse priming effects differed across the four masks. Effects were indistinguishable for the orthogonal, the diagonal and the arrow pattern mask but were larger for the superposition mask at the 100 and 200-msSOA.

Figure 3. Scatterplots illustrating the relationship of prime visibility and priming effects for the orthogonal, diagonal, arrow pattern and superposition mask. Within each panel, data for each SOA are depicted separately. Priming effects were independent of prime visibility for the superposition mask whereas both measures were correlated for the other masks. We performed a two-means cluster analysis on the prime visibility data which revealed a group of participants showing supraliminal prime perception and no priming effects and another group showing subliminal prime perception and inverse priming effects. 
Figure 1
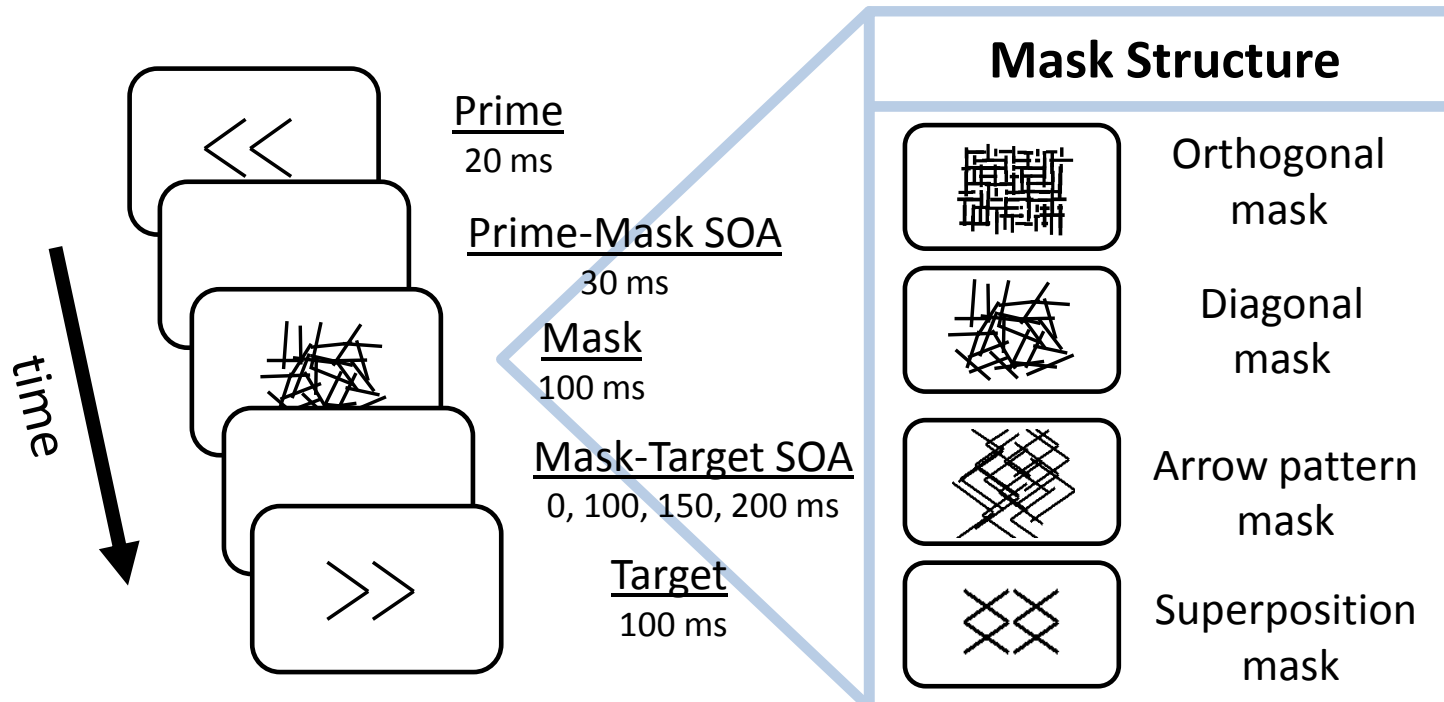
Figure 2

A

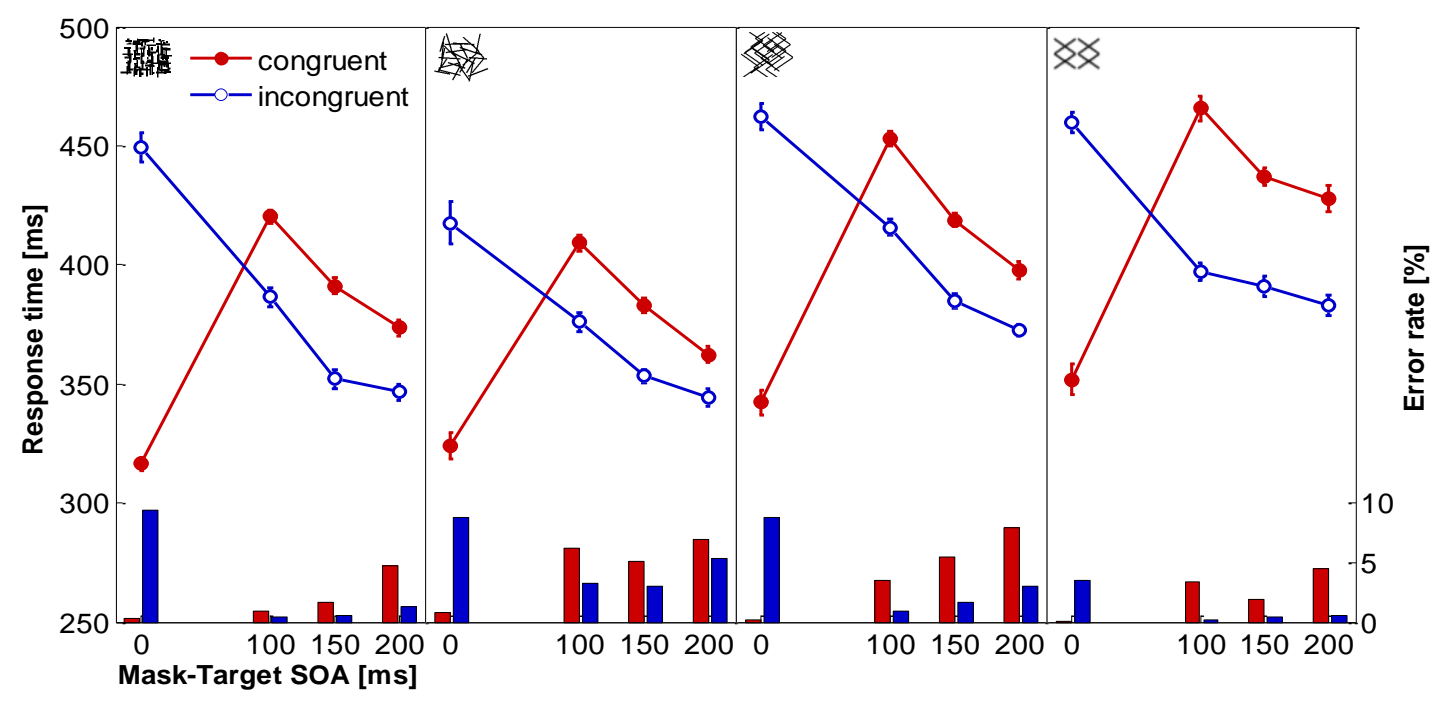

B

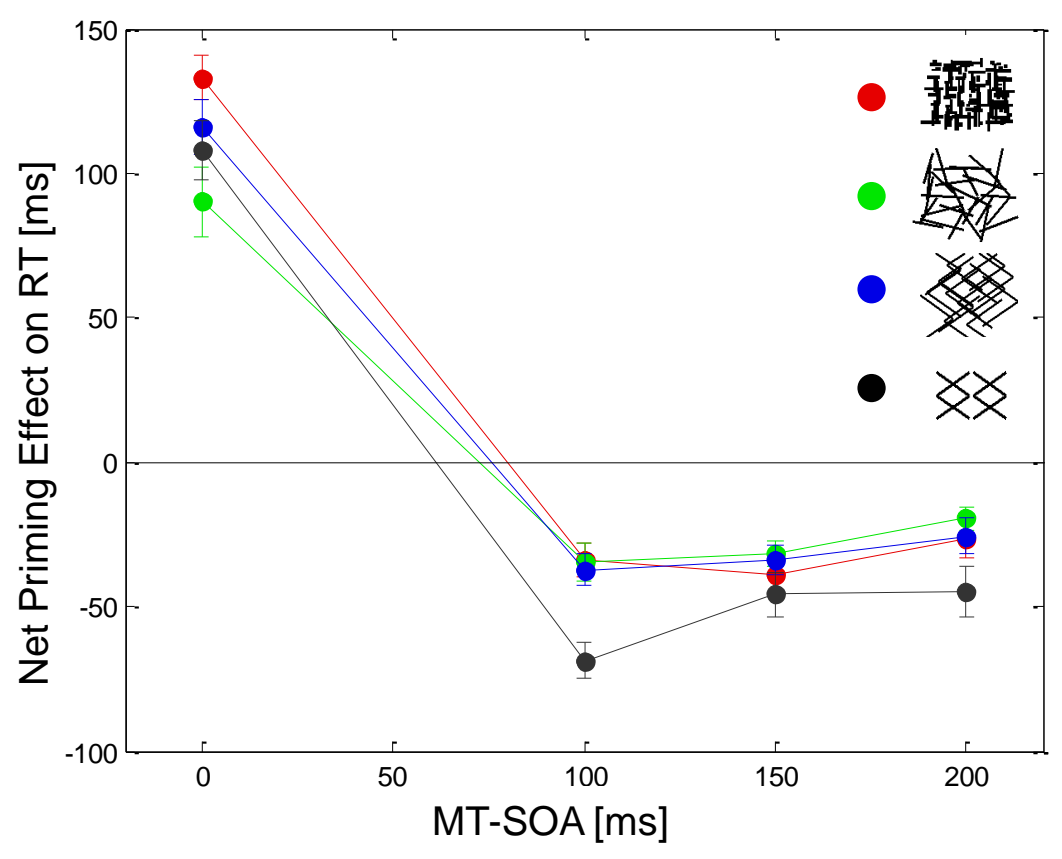


Figure 3

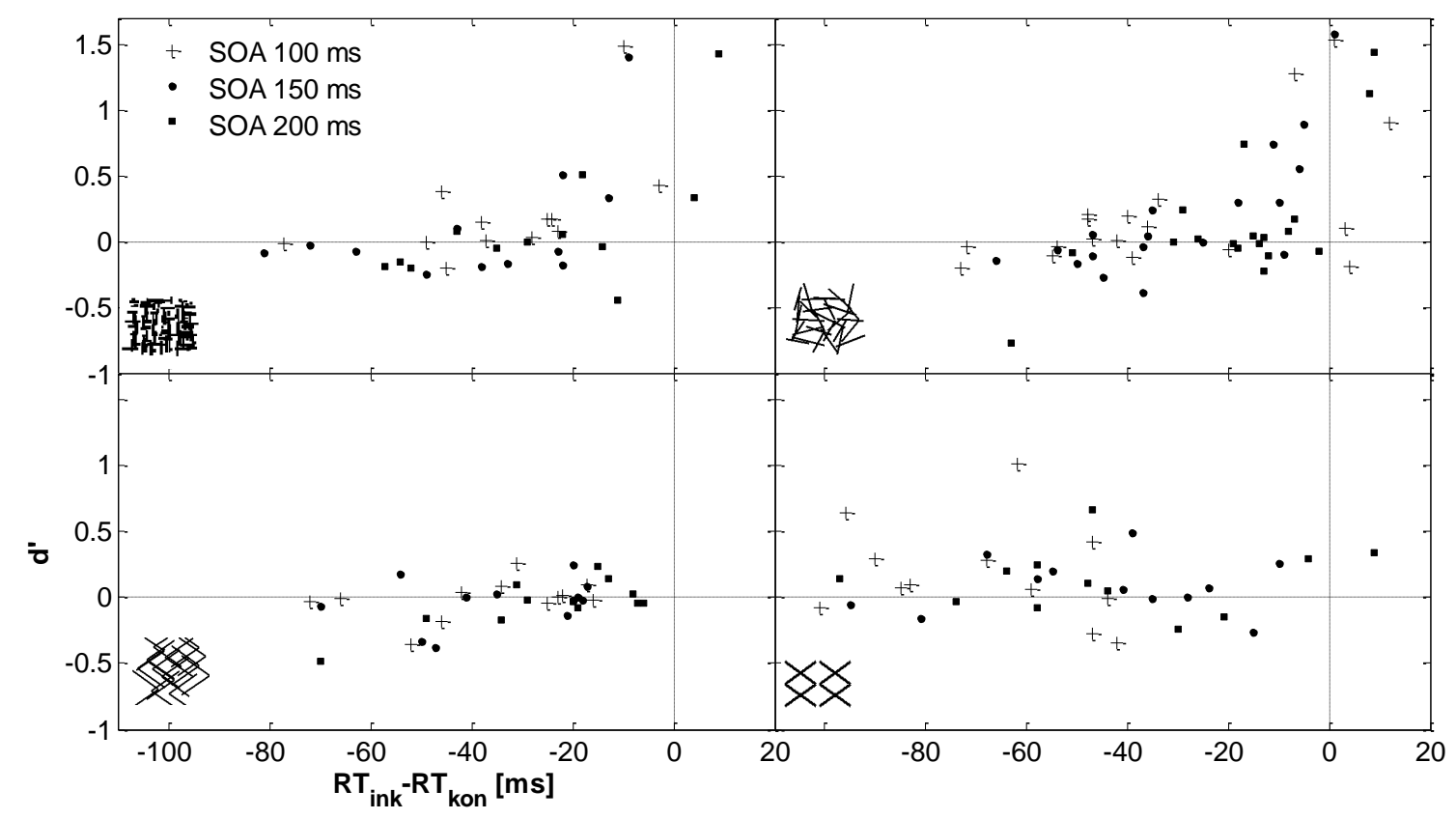




\section{Appendix III}

Krüger, D., \& Mattler, U. (in prep.). The role of stimulus-response compatibility in inverse priming. 


\title{
The role of stimulus-response compatibility in inverse priming
}

\author{
Daniel Krüger \& Uwe Mattler
}

Georg-Elias-Müller Institute for Psychology, Georg-August University Göttingen

Send correspondence to:

Uwe Mattler

Georg-Elias-Müller Institute for Psychology

Georg-August University Göttingen

Gosslerstr. 14

D-37073 Göttingen

Germany

Email: uwe.mattler@psych.uni-goettingen.de 


\begin{abstract}
(250 words)
Inverse priming is a counterintuitive reversal of priming effects with better performance on incongruent trials, which seems to be due to the presentation of a separate masking stimulus following the prime but preceding the target. When the masking stimulus contains taskrelevant features a perceptual interaction with the prime could readily explain inverse priming. In contrast, inverse priming with neutral masks free of target-like elements has been explained by inhibition of the primed response due to mask presentation triggered by a motor control mechanism. Here, we studied the locus of inverse priming with such so-called irrelevant masks. We investigated whether inverse effects of masked double arrow primes also extend to physically dissimilar targets. We observed a full transfer of the effect providing further evidence for a motor account of inverse priming with arrow stimuli. In contrast, however, in eight further experiments, we failed to find any evidence for inverse priming employing non-arrow prime and target stimuli. Converging evidence supports the idea that stimulus-response compatibility is crucial for inverse priming to occur with irrelevant masks. Such stimuli might directly affect motor preparation and thereby trigger motor inhibition due to mask presentation. Response non-compatible stimuli, in contrast, are subjected to a controlled response selection process and do not directly activate motor responses. Consequently, such stimuli fail to provoke inverse priming when simple perceptual interactions of the stimuli are excluded as a potential source of the effect by using irrelevant masks.
\end{abstract}




\section{Introduction}

Masked prime stimuli can affect overt behavior even when they are not consciously accessible. Masked priming effects are typically demonstrated on the speeded discrimination of a following target stimulus. In most cases, performance benefits are observed whenever primes share some response-critical feature with the target stimulus (congruent condition) and costs when they share features with the alternative target (incongruent condition). Apart from such positive priming effects (e.g. Mattler, 2003), a counterintuitive reversal of priming effects termed inverse priming or the negative compatibility effect (NCE) is often found (e.g. Eimer, \& Schlaghecken, 1998). The boundary conditions for this phenomenon are still a matter of discussion. In most cases, a separate masking stimulus presented after the prime but prior to the target led to the reversal. This mask is considered as a trigger for the reversal to occur by the majority of theoretical accounts on inverse priming (Klapp, \& Hinkley, 2002; Lleras, \& Enns, 2006; Jaśkowski, \& Przekoracka-Krawczyk, 2005; Schlaghecken, \& Eimer, 2002; but see: Klauer, \& Dittrich, 2010). The design of the mask itself modulated the strength of the reversal. Inverse priming has been found more pronounced with masks containing target-like features (e.g. Jaśkowski, 2007, 2008; Klauer, \& Dittrich, 2010; Lleras and Enns, 2004, 2005, 2006; Schlaghecken and Eimer, 2006). Object Updating accounts for this relative increase of inverse priming effects with such so-called relevant masks. The core assumption is a perceptual interaction of prime and mask increasing saliency for those elements of the mask dissimilar to the prime which in turn facilitates processing of incongruent targets whenever masks are built from both prime alternatives (Lleras, \& Enns, 2004). Object Updating, however, is not an exhaustive explanation for all instances of inverse priming because the effect is still obtained with masks entirely free of target-like elements (Eimer, \& Schlaghecken, 2002; Klapp, 2005; KLapp, \& Haas, 2005; Lleras, \& Enns, 2005; Schlaghecken, \& Eimer, 2006; Schlaghecken, Rowley, Sembi, Simmons, \& Whitcomb, 2007). A broad range of theoretical explanations have been proposed for inverse priming with these so called irrelevant masks. Various researchers supported the idea of a motor control mechanism triggered by the mask which counteracts prime elicited response activation (Lleras, \& Enns, 2006; Jaśkowski, \& Przekoracka-Krawczyk, 2005; Schlaghecken, \& Eimer, 2002). In contrast, recently various alternative accounts have been proposed challenging the idea of mask-triggered motor inhibition. Some of these theories ascribe inverse priming to more central levels of processing, others postulated a perceptual source of the effect. 
Perceptual accounts explain the relative performance costs on congruent trials by hampered perceptual processing of congruent targets either due to habituation (Huber, 2008) or a conflict-sensitive attentional refractory period (Sohrabi, \& West, 2009). In contrast, adherents of a more central source of inverse priming effects, claimed that masked (arrow) primes induce an automatic shift of spatial attention according to the prime's orientation which is, however, immediately compensated in order to reorient attention to the centrally presented target stimulus. The direction of backward moving attention supports response selection for incongruent but interferes for congruent targets leading to inverse priming (Cole, \& Kuhn, 2010). Alternatively, Klauer and Dittrich (2010) proposed an evaluation window account of inverse priming which postulates two accumulators located at an abstract response selection level where incoming information is assigned to the task-relevant categories (left and right). These accumulators collect evidence for either response during an evaluation window. In the typical inverse priming paradigm, the mask serves as a go-signal triggering the evidence monitoring process in anticipation of the target. Consequently, prime stimuli are excluded from the evaluation window in case of inverse priming. However, primes fed the accumulators before leading to a bias at the onset of the evaluation window which hampers selection of a prime-congruent response because response selection is based on the relative increase rate which is higher for the unprimed accumulator which is still in its initial state. In contrast, in positive priming the prime itself is the go-signal for the evaluation window to start and thus the prime is included in the evaluation process biasing response selection towards the primed alternative.

The aforementioned accounts do not claim to fully capture all instances of inverse priming. These accounts are rather not mutually exclusive. The spatial attention account (Cole, \& Kuhn, 2010), for example, is clearly limited to prime stimuli known to produce a reflexive shift of spatial attention and thus seems to be unable to account for inverse priming with nonarrow stimuli (Schlaghecken, et al., 2007). Numerous studies suggest that different sources contribute to inverse priming dependent on e.g. the mask structure (e.g. Lleras, \& Enns, 2004) or the prime and target stimuli employed (Jaśkowski, \& Ślósarek, 2007).

Consequently, evidence from studies seeking to localize inverse priming effects led to divergent results. Support for a perceptual account comes from a recent study which combined the inverse priming task with a secondary task in a Psychological Refractory Period paradigm. Assessment of the interference effects between both tasks consistently suggested a purely perceptual source of inverse priming effects (Krüger, Klapötke, \& Mattler, 2011). 
Further support is derived from a study by Mattler (2006) who disentangled the contribution to inverse priming which is due to visual similarity of primes and targets (i.e. a perceptual contribution) from that of their congruency (post-perceptual contributions). He found that the entire effect results from the perceptual similarity. Supporting the same idea, inverse priming effects have been found to extend to the priming of non-motor operations which seems to obey to similar laws as motor priming suggesting a common mechanisms producing both (Mattler, 2007, within the domain of positive priming see also: Mattler, 2003). In a non-motor priming paradigm, primes are completely dissociated from motor output. Consequently, primes cannot affect motor processes. However, all these studies in favor of a perceptual account of inverse priming employed meta-contrast masks which entail task-relevant features raising the question of the generalizability of these results to irrelevant masks.

Moreover, there is also a bulk of evidence supporting the notion of a motor origin of inverse priming effects. Electrophysiological studies, for instance, demonstrate that inverse priming is reflected in lateralized readiness potentials (LRP, Eimer, \& Schlaghecken, 1998, 2003; Praamstra, \& Seiss, 2005) which is considered to be an index of response preparation processes. These results are further supported by some more recent studies demonstrating a crucial involvement of the supplementary motor area (SMA) in inverse priming (Boy, Evans, et al., 2010; Boy, Husain, et al., 2010; Sumner, et al., 2007). This area within the dorsal part of the medial frontal lobe has been found involved in motor control processes (for a review see: Nachev, et al., 2008). However, one has to keep in mind that these physiological correlates of inverse priming can be secondary consequences from earlier, e.g. perceptual, processes that simply translate to the motor system. In contrast, demonstrations of inverse priming effects on free-choice responses (Klapp, \& Haas, 2005; Klapp, \& Hinkley, 2002, Schlaghecken, Klapp, \& Maylor, 2009) impose more serious constraints for a perceptual account because even though target stimuli are perceptually dissimilar response choices are biased towards the unprimed response. Maybe the strongest evidence for a motor locus has been presented sofar by Boy and Sumner (2010) who demonstrated that an unexpected reversal of arbitrarily chosen stimulus-response (SR) mappings led to a full reversal of priming effects (inverse effects became positive and positive effects became inverse). From a motor perspective, the reversal is due to the learned association of the stimuli with responses when the reversal of SR-mappings occurs. In concrete terms: A congruent prime associated through learning with the left-response leads to a motor bias towards a right response after mask-induced inhibition occurred which is the correct response to a congruent target after the reversal of SR-mappings. A perceptual account, however, predicts unchanged priming effects 
because the similarity relation is unaffected by the reversal of SR-mappings: performance should be still worst on congruent trials even after the reversal occurred.

In contrast to the reviewed findings, some evidence would favor an origin at more central processing levels where abstract response categories are represented instead of concrete motor commands. Schlaghecken, Münchau, Bloem, Rothwell and Eimer (2003) applied repetitive transcranial magnetic stimulation (rTMS) to the primary motor cortex. While TMS altered overall response speed it failed to influence inverse priming effects suggesting that the effects does not originate from M1. Furthermore, Schlaghecken, Klapp and Maylor (2009) demonstrated an inverse effect of primes on the performance with an additional perceptually dissimilar target stimulus when it required withholding to respond (NoGo condition) or choosing either response (Free-Choice condition) but not when it required bimanual responses. The fact that bimanual responses were not led by the hand opposite to the prime's direction suggests that primes affect more abstract directional representations not individual muscle commands.

A straight-forward account to isolate perceptual from post-perceptual contributions to inverse priming has been developed by Eimer (1999). He studied to what degree inverse priming effects translate to perceptually dissimilar targets. To this end, double arrow targets (identical to the primes) were intermixed with lateral '+' -signs which, thus, differed from primes with respect to their geometrical shape and location. Eimer (1999) found that priming effects on response times fully propagated to such perceptually dissimilar targets conflicting with a perceptual source of the effect. Later on, Klapp and Hinkley (2002) extended these findings by showing a transfer of priming effects to a different stimulus modality. Reversed effects of visual primes were equally large for visual and auditory targets. In contrast, two studies failed to find such a transfer of priming effects to perceptually dissimilar targets for different reasons. Schlaghecken and Eimer (2000) found that inverse priming was limited to identical targets which is at odds with Eimer's fingings (1999). To account for this difference, Schlaghecken and Eimer (2000) argued that motor inhibition could not fully develop for lateral targets because responses to lateral targets were much faster (which was not the case in Eimer, 1999). In line with this interpretation, inverse priming was found for lateral targets when only considering the slowest responses of each participant $\left(60^{\text {th }}\right.$ and $80^{\text {th }}$ percentile of RT distribution). Eimer, Schubö and Schlaghecken (2002) also studied priming effects for identical targets combined with dissimilar ones. However, in contrast to the above mentioned studies, lateral targets required responding with a different effecter (hands or feet, 
respectively). No transfer of priming effects was found which led the authors to conclude that inverse priming is effecter-specific disregarding the possibility that perceptual similarity is crucial for the effect to occur.

Thus, we then have evidence from at least two studies investigating the transfer of inverse priming to dissimilar targets for a motor locus of the effect (Eimer, 1999; Klapp, \& HInkley, 2002, and with limitations also: Schlaghecken, \& Eimer, 2000). However, all these studies employed an at least potentially relevant mask. While Eimer (1999) and Schlaghecken and Eimer (2000) constructed the mask by simply superimposing both prime alternatives, Klapp and Hinkley (2002) used letter masks also containing arrow-elements. Therefore, it is possible that the transfer of the effects is completely due to special design of the mask. Its presentation after the prime could generate the impression of adding the alternative prime. The onset of the prime-opposite stimulus might then facilitate responding to a lateral target appearing at the location pointed at by this "second prime arrow". To be confident about a motor locus of inverse priming, the transfer of priming effects should be demonstrated with a mask that excludes the possibility of such perceptual interactions with the prime. When inverse priming with such an irrelevant mask is due to the inhibition of the primed motor response the effect should also become manifest for perceptually dissimilar targets just sharing the same responses. In contrast, to the degree that priming effects to perceptually dissimilar targets are diminished perceptual similarity contributes to the effect. These hypotheses were tested in the current experiments.

Please insert Figure 1 about here 


\section{Experiment 1}

To investigate to what degree perceptual processes contribute to inverse priming with irrelevant masks we compared responses to centrally presented double arrows with responses to lateral target stimuli. The central targets were identical to the primes while the lateral targets were perceptually dissimilar. When inverse priming is indeed a motor phenomenon and the primed response is inhibited then equal effects on responses to both types of targets are expected. However, to the degree that perceptual processes contribute, effects of the prime should be reduced for the dissimilar lateral targets.

\section{Method}

Participants. 30 students from the University of Göttingen participated in Experiment 1 (6 males, mean age 23.4 years, std 2.8 years, range 19-31 years). All but three were right-handed by self-report, and all of them had normal or corrected-to-normal vision. Each participant received $17.50 €$ for participation in the two sessions of the experiment.

Tasks. (a) Choice-RT task: In the first session, participants were required to make speeded responses to a target stimulus which was a centrally presented double arrow on half of the trials. Double arrows could either point left- or rightwards (see Figure 1). On the remaining trials, a '+'-sign appeared either on the left-hand side or right-hand side of the screen. Leftward pointing double arrows and a cross located on the left-hand side required a button press with the index finger of the left hand, rightward-pointing arrows and a cross on the right-hand side with the index finger of the right hand. (b) Prime-recognition: Primes were always centrally presented double arrows which also pointed either left- or rightwards. In the second session participants were informed about their presence and they should report the prime orientation with the same response keys but without speed stress.

Stimuli. Primes were identical to the double arrows used as target stimuli. On half of the trials, primes required the same response as the target stimuli (congruent condition) and required opposite responses on the other trials (incongruent condition). Primes were followed by a masking stimulus also presented at the screen centre (see Figure 1). A new mask was generated for each trial to avoid perceptual learning which might aid in prime recognition (Schlaghecken, Blagrove, \& Maylor, 2008). Masks were composed of 130 line elements centred on a 13 x 10 grid, including a spatial jitter. All stimuli were presented in black color on a white background. Double arrows subtended $1.5^{\circ} \times 1^{\circ}$ of visual angle. Random line masks subtended $1.7^{\circ} \times 1.4^{\circ}$, the length of individual lines ranged from $0.06^{\circ}$ to $0.45^{\circ}$ and 
their width from $0.08^{\circ}$ to $0.16^{\circ}$. Lateral ' + '-signs subtended $0.6 \times 0.6^{\circ}$ and were presented with an eccentricity of $4.6^{\circ}$. During both sessions, incorrect responses were signaled by visual error feedback (translated from German: "Error").

Procedure. Participants were tested individually on separate days. In a dimly lit room they sat $100 \mathrm{~cm}$ in front of a computer monitor with the chin fixed in a rest. Both sessions started with instructions and a practice section. In the first session, participants were familiarized with the target stimuli and performed a block of 48 demo-trials which was identical to those of the experiment. At the beginning of the second session, participants were told about the presence of prime stimuli and they were shown the entire stimulus sequence with long stimulus durations to ensure that they understood the prime recognition task. Participants were instructed to identify prime stimuli as accurately as possible without speed stress. Apart from this initial part, both sessions were identical and comprised 12 blocks with 48 trials each.

Stimuli and timing parameters were identical across both sessions. A central fixation cross indicated the start of each trial. After $500 \mathrm{~ms}$ it disappeared and was followed after $300 \mathrm{~ms}$ by the prime shown for $20 \mathrm{~ms}$. After a prime-mask SOA of $30 \mathrm{~ms}$ the mask was presented for $100 \mathrm{~ms}$. Targets were also presented for $100 \mathrm{~ms}$ and followed the mask with a mask-target SOA of $150 \mathrm{~ms}$. In the first session, the computer monitored for a response for $1800 \mathrm{~ms}$ while during prime recognition response latencies were unlimited. After response delivery the next trial started after a variable inter-trial-interval (range 800-2050 ms, mean ca. $1115 \mathrm{ms,}$ pseudo-exponentially distributed).

Apparatus. The experiment was run on a personal computer connected to a CRT-display (100 $\mathrm{Hz}$ vertical refresh rate, resolution 1024 x 768). During all three sessions, response buttons were the left and right CTRL-buttons of the computer keyboard.

Design. (a) Choice-RT task: During the first session, a two-factorial repeated measures design with the factors Target Type (central vs. lateral) and prime-target Congruency (congruent vs. incongruent) was implemented. Response times and error rates served as dependent measures. 12 Blocks of 48 trials were acquired in each session yielding 144 replications for each of the 4 experimental conditions resulting from the factorial combination of SOA and Congruency. (b) Prime recognition performance was measured by the proportion of correctly reported primes differentiating only for Target Type. Thus, prime visibility estimates were based on 288 trials for each Target Type. 
Data Analysis. Prior to statistical analysis of RT data, error and post-error trials were excluded. Trimmed means were built by discarding the two fastest and three slowest responses for each participant and condition. Error rates were arc-sine transformed. RT and error rates data were then subjected to repeated measures ANOVAs for the factors Target Type and Congruency. To further assess propagation of priming effects to lateral targets correlations of priming effects between central and lateral targets were computed.

Results

$R T$. Target Type affected RTs, $\mathrm{F}(1,29)=13.5, \mathrm{p}<.0001$ (see Figure 2). Reponses to the lateral targets were faster $(361 \mathrm{~ms})$ as opposed to central double arrows (376 ms). Priming effects differed between these two target types as indexed by the significant two-way interaction, $\mathrm{F}(1,29)=4.7, \mathrm{p}<.05$. With both types of target stimuli, inverse priming effects were obtained but these were larger with the central targets $(-23 \mathrm{~ms}, \mathrm{t}(29)=-3.5, \mathrm{p}<.01)$ as compared to lateral targets $(-15 \mathrm{~ms}, \mathrm{t}(29)=-3.2, \mathrm{p}<.01)$. Priming effects for central and lateral targets were correlated $(r=.83, p<.001$, cf. Figure 2$)$.

Please insert Figure 2 about here

Accuracy data. Errors were committed on $3.5 \%$ of all trials (see Figure 2). Errors occurred more frequently with central $(5.6 \%)$ as compared to lateral targets $(1.4 \%), \mathrm{F}(1,29)=102.0, \mathrm{p}$ $<.0001$. Priming effects differed for the two targets, $\mathrm{F}(1,29)=12.0, \mathrm{p}<.01$. For the lateral targets, priming effects did not differ significantly from zero, $+0.9 \%, \mathrm{t}(29)=-1.5, \mathrm{p}<.14$. In contrast, for central targets incongruent primes led to fewer errors resulting in an inverse priming effect, $-2.2 \%, \mathrm{t}(29)=2.1, \mathrm{p}<.05)$. Priming effects to central and lateral targets were correlated $(\mathrm{r}=.44, \mathrm{p}<.05$; see Figure 2$)$

Please insert Figure 3 about here 
Prime Recognition. Participants correctly reported prime orientation on $59.4 \%$ of all trials, which is significantly better than chance level, $\mathrm{t}(29)=3.1, \mathrm{p}<.01$. Recognition performance was not affected by Target Type, $\mathrm{t}(29)=.4, \mathrm{p}=.72$. However, across participants prime recognition performance varied substantially: while worst performance in our sample was around chance level (44.2\% correct averaged across both Target types), best performance reflected almost unimpaired recognition of the primes $(96.0 \%)$. This substantial interindividual variability and the notion that inverse priming has been found to depend on prime visibility (for a review see: Sumner, 2007) led us to test whether priming effects changed with prime visibility.

Prime recognition and priming effects for central targets. In a first attempt, we calculated correlation coefficients to estimate a linear relation of prime recognition measures and priming effects on response times and error rates for the central targets. For both dependent measures, a significant positive correlation was observed $(\mathrm{RT}: \mathrm{r}=.49, \mathrm{p}<.01$; accuracy data: $\mathrm{r}=.39, \mathrm{p}<.05)$. The relationship is illustrated in Figure 4A. Visual inspection of the scatterplots, however, does not suggest a continuous linear relationship but rather the existence of two distinct groups of participants (for a similar interpretation see also: Sumner, 2007). While the majority of participants showed chance-level performance in prime recognition, few subjects were clearly better. Apparently, these two groups also differed with respect to priming effects: while the distribution of priming effects seems to be clearly shifted towards inverse effects for those subjects with poor prime recognition, good prime recognition seems to be accompanied by priming effects scattered around zero. Therefore, we aimed to compare priming effects between these two groups. However, determining a criterion to distinguish subliminal from supraliminal perception is necessarily arbitrary (Kouider, \& Dehaene, 2007). Therefore, we chose two criteria derived from two different approaches. In a data-driven approach, we entered prime recognition performance of a total of 128 subjects from all those experiments in the current study that include lateral target stimuli (i.e. all but Experiment $4 \mathrm{~b}$ and 9) in a two-means cluster analysis. As a criterion we chose that value which best differentiates between the two groups built by the cluster analysis which is the mean between the maximum prime recognition among the poor performance group and minimum among the good performance group. The resulting critical value was $63.9 \%$. In a second account, we derived a criterion from the theoretical distribution of prime recognition 
performance at chance-level. We assumed that when subjects were guessing during prime recognition the percentage of correct responses should follow a binomial distribution with $\mathrm{N}$ $=576$ trials and a probability for a correct response of $\mathrm{p}=.5$. Chance-level performance is rather unlikely $(\mathrm{p}<.05)$ when prime recognition performance exceeded $53.5 \%$ of correct responses. Thus, we obtained a rather strict criterion from these theoretical considerations (53.5\%) compared to the empirical criterion given by the cluster analysis (63.9\%). However, results of the comparison of priming effects between good versus bad recognition performance were essentially the same for these two criteria confirming the impression from the visual inspection of Figure 4: When groups were built according to the theoretical criterion, priming effects on $\mathrm{RTs}, \mathrm{F}(1,28)=8.3, \mathrm{p}<.01$, and error rates, $\mathrm{F}(1,28)=5.9, \mathrm{p}<.05$ differed among the two visibility groups. While the group with poor prime recognition showed inverse priming effects on RTs, $-39 \mathrm{~ms}, \mathrm{t}(15)=4.5, \mathrm{p}<.001$, and error rates, $-4.1 \%$, $t(15)=2.8, p<.05$, no priming effects were observed for the good prime recognition group, neither on RTs, $-5 \mathrm{~ms}, \mathrm{t}(13)=.6, \mathrm{p}=.53$, nor on error rates, $0.0 \%, \mathrm{t}(13)=-.3, \mathrm{p}=.80$. When groups were built according to the empirical criterion, priming effects depended on prime visibility with respect to RT data, $\mathrm{F}(1,28)=7.5$, $\mathrm{p}<.05$, and error rates, $\mathrm{F}(1,28)=5.3$, $\mathrm{p}<$ .05. Inverse priming effects on RTs, $-33 \mathrm{~ms}, \mathrm{t}(21)=4.5, \mathrm{p}<.001$, and error rates, $-3.9 \%$, $\mathrm{t}(22)=3.0, \mathrm{p}<.01$, were observed for the poor prime recognition group and no priming effects on RTs, $+4 \mathrm{~ms}, \mathrm{t}(7)=-.4, \mathrm{p}=.68$, as well as error rates $,+2.5 \%, \mathrm{t}(7)=-.8, \mathrm{p}=.41$, for the good performers (Figure 4B).

\section{Discussion}

In accordance with previous research employing the same paradigm with relevant masks (Eimer, 1999; Klapp, \& Hinkley, 2002), we found evidence for the idea that inverse priming truly reflects inhibition of motor responses even when irrelevant masks are used because priming effects were also observed for perceptually dissimilar targets which were spatially separated from the primes. Across subjects, the magnitude of priming effects on central targets was strongly related to the magnitude of the effect on lateral targets suggesting that the same motor processes occur irrespective of which target followed. However, in contrast to the aforementioned studies, we obtained a clear reduction of the inverse priming effects with lateral targets pointing towards a non-motor part of the effect which is due to the perceptual similarity of primes and targets. A similar reduction of inverse priming effects to lateral has also been observed before (Eimer, Schubö, \& Schlaghecken, 2002). In this study, priming 
effects completely failed to transfer to lateral targets which, however, required responding with a different response modality as opposed to responses to central targets. In the light of the prior results by Eimer (1999) the authors completely ruled out a perceptual source of inverse priming as an explanation for the absence of effects to lateral targets and instead concluded that inhibition of prime-induced activity is effecter-specific. A further study also found that inverse priming effects disappeared with lateral targets (Schlaghecken, \& Eimer, 2000). The authors attributed the absence of inverse priming effects to the overall shorter response latencies for lateral targets. They argue that the prime-response interval is crucial for the magnitude of inverse priming effects because when response latencies are rather short responses are selected during the initial activation phase exclusively governed by the prime. In line with these considerations, only the slowest responses to lateral targets $\left(60^{\text {th }}\right.$ and $80^{\text {th }}$ percentile) showed reliable inverse priming. In the light of this finding, we performed the same analysis on our data. We split our data into RT bins $\left(20^{\text {th }}, 40^{\text {th }}, 60^{\text {th }}, 80^{\text {th }}\right.$ percentile $)$ and analyzed whether priming effects depended on absolute response speed for central and lateral targets by means of a repeated measures ANOVA with the factors Percentile, Congruency and Target Type. Priming effects depended on Percentile, $F(3,87)=6.9, p<.001$. This dependency further differed between central and lateral targets as indexed by the three-way interaction Percentile $x$ Congruency $x$ Target Type: $F(3,87)=5.7, p<.01$. When analyzed alone, priming effects to central target did not differ for the percentiles considered, $\mathrm{F}(3,87)=$ $1.0, \mathrm{p}=.38$ (effects were $-22,-27,-26,-27 \mathrm{~ms}$, for the $20^{\text {th }}, 40^{\text {th }}, 60^{\text {th }}, 80^{\text {th }}$ percentile, respectively), but they clearly did for lateral targets, $\mathrm{F}(3,87)=11.0, \mathrm{p}<.0001$. In accordance with the results presented by Schlaghecken and Eimer (2000), priming effects seemed to increase with increasing response latencies (-6, -14, -20, -24 ms). To assess to what degree effects transferred to lateral targets, we compared priming effects for each percentile between central and lateral targets. Note that the absolute response speed in each Percentile condition of course differed for both targets. Priming effects differed between both targets for the $20^{\text {th }}$ and $40^{\text {th }}$ percentile, Congruency $x$ Target Type, $F(1,29)=14.7$ and $7.1, p<.001$ and $<.02$, but were indistinguishable for the $60^{\text {th }}$ and $80^{\text {th }}$ percentile, $F(1,29)=2.3$ and $0.5, p=.14$ and .48 . Thus, our analyses confirmed the assumption put forward by Schlaghecken and Eimer (2000). When correcting for absolute response speed by considering only slowest responses, priming effects completely transferred to lateral targets suggesting that the motor control processes leading to inverse priming require some time to fully develop. If so, however, why were priming effects to central targets not influenced by response speed? Because the analysis reported above was not fine-grained enough. When augmenting the RT bin resolution, we 
found the same tendency. When considering $5^{\text {th }}$ to $95^{\text {th }}$ percentile in steps of $5 \%$, priming effects clearly depended on Percentile also for central targets, $F(18,522)=3.8$, $p<.0001$. Inverse priming effects were small an insignificant for the fastest responses $\left(5^{\text {th }}\right.$ and $10^{\text {th }}$ percentile: $\mathrm{t}(29)<-1.6, \mathrm{p}>.13$ ) but clearly found for slower responses (all other percentiles: $\mathrm{t}(29)<-2.1, \mathrm{p}<.04)$. In sum, these additional analyses provide evidence for the view that inverse priming effects depend on absolute response speed and that the effects seem to be entirely due to motor processes when taking the response speed differences between central and lateral targets into account.

Apart from the question of the origin of inverse priming effects we examined the relation between priming effects and prime visibility. We found that primes only affected task performance within those subjects being unable to perceive it in the latter prime recognition session. A minority of subjects possessed an extraordinary capacity to recognize prime stimuli. Within these subjects, no effect of the prime was found. The influence of prime visibility on priming effects has been demonstrated in several prior studies. Klapp (2005; Klapp and Hinkley, 2002) compared priming effects for different letter masks and reported larger inverse priming effects for more effective masks. Similarly, Eimer and Schlaghecken (2002) altered prime visibility by manipulating prime duration and in another experiment mask density. They observed a remarkable correspondence between the individual transition point from sub- to supraliminal prime perception and whether priming effects were positive or inverse. Along the same lines, Sumner, Tsai, Yu and Nachev (2006) manipulated prime duration and found positive effects of visible primes and inverse effects of invisible ones. As in the current experiment, priming effects across different subjects with varying prime recognition capacities could also be compared while stimulus conditions are kept constant. Applying this account, Sumner (2007) reported positive priming for subjects consciously perceiving the prime and inverse priming for the remaining participants. In sum, all these data demonstrate more inverse effects for primes rendered invisible and more positive effects for supra-threshold primes. Thus, at first glance, these data suggest a causal role of prime visibility for inverse priming effects. In contrast to this interpretation, other recent data demonstrated inverse priming with "masking stimuli" that do not spatially overlap the primes thus leaving them perfectly visible (Jaśkowski, 2007; 2008; Jaśkowski, Biłuńska, Tomanek, \& Verleger, 2008; Lleras, \& Enns, 2006). Furthermore, Schlaghecken and colleagues found a dissociation of priming effects from prime visibility in a perceptual learning experiment: while prime recognition performance systematically improved across five learning sessions, inverse priming effects remained unchanged (Schlaghecken, Blagrove, \& Maylor, 2008). 
Therefore, it seems more reasonable to understand prime visibility as a moderating factor for the size of inverse priming effects instead of considering it as a necessary prerequisite for the effect. Experimental manipulations within the same individual on the one hand or preexisting inter-individual differences on the other hand might affect both, prime visibility and priming effects, at the same time.

Coming back to the initial question concerning the locus of inverse priming, we observed a reduction of priming effects for lateral targets which, however, disappeared when compensating for the higher response speed to these targets by considering only slowest responses. These data are in line with previous evidence from the same paradigm applied to relevant masks (Eimer, 1999; Klapp \& Hinkley, 2002). Because the current experiment is the only empirical attempt addressing inverse priming with irrelevant masks we intended to replicate the findings in Experiment 2 using different prime and target stimuli.

\section{Experiment 2}

Experiment 2 was identical to Experiment 1 except for the design of the prime stimuli which again also appeared as central target stimuli on half of the trials. Masks were identical random line masks as employed in Experiment 1. In order to keep the similarity of mask features to the new prime stimuli comparable to Experiment 1, we rearranged the elements of the double arrows yielding two vertically aligned arrows pointing up and down which were either directed away from each other or towards each other (see Figure 1). According to our results from Experiment 1, we expected to replicate inverse priming effects for central targets and the partial transfer to lateral targets.

\section{Method}

Participants. 14 new students from the University of Göttingen participated in Experiment 2 (3 males, mean age 24.1 years, std 3.4 years, range 20-33 years). All but one were righthanded by self-report, and all of them had normal or corrected-to-normal vision. Each participant received $24.50 €$ for participation in the three sessions of the experiment.

Task, Stimuli, Procedure, and Design. Experiment 2 replicated Experiment 1 using different prime and central target stimuli. These new stimuli consisted in a vertically aligned pair of 
arrows which was built from the same elements as the double arrows used in Experiment 1. When the arrows pointed towards each other a left-hand response was required and when they pointed away from each other a right-hand response was required. Both stimuli subtended $1.2^{\circ} \times 1.1^{\circ}$. Again, central targets were interspersed with lateral targets which were identical ' + '-signs as in Experiment 1. Because stimulus-response relations for the central targets were non-compatible and thus had to be learned first, subjects performed two Choice-RT sessions before the final prime recognition session. Therefore, we included Session as an additional factor in the analysis yielding a three-factorial repeated measures design.

Results

RT. As depicted in Figure 5, Target Type affected RTs, $\mathrm{F}(1,13)=40.6, \mathrm{p}<.0001$. Participant's responses were faster for lateral $(382 \mathrm{~ms})$ than for central targets $(501 \mathrm{~ms})$. Overall RTs also differed across the two sessions, $\mathrm{F}(1,13)=31.3, \mathrm{p}<.0001$. Participants responded slower in the first $(474 \mathrm{~ms})$ as compared to the second session (409 ms). This learning effect differed between the two Target Types, $\mathrm{F}(1,13)=8.4, \mathrm{p}<.05$. RTs decreased by $77 \mathrm{~ms}$ for central targets and by $53 \mathrm{~ms}$ for lateral targets. Contrary to our expectations, RTs were not affected by Congruency, $\mathrm{F}<1$. The other effects also failed to reach significance.

Accuracy data. On average, participants committed an error on $2.5 \%$ of all trials. The error rate was influenced by Target Type, $\mathrm{F}(1,13)=49.6, \mathrm{p}<.0001$. More errors were made in response to central $(4.6 \%)$ as compared to lateral $(0.4 \%)$ target stimuli. Importantly, the error rate was not affected by Congruency, $\mathrm{F}(1,13)<1$. No other effect reached significance.

Please insert Figure 5 about here

Prime recognition. Prime recognition performance was influenced by Target Type, $\mathrm{t}(13)=-$ $2.6, \mathrm{p}<.05$. The percentage of correctly reported primes was larger for lateral $(55.3 \%)$ than for central targets $(52.4 \%)$. When tested against chance level, performance turned out to be significantly above chance level for lateral targets, $\mathrm{t}(13)=2.9, \mathrm{p}<.05$, but not for central targets, $\mathrm{t}(13)=1.5, \mathrm{p}=.17$. Prime recognition performance varied across individuals and 
ranged from $46.5 \%$ to $66.7 \%$ for central targets and from $47.6 \%$ to $69.4 \%$ for lateral targets. Next, we explored whether priming effects on central targets depended on prime visibility as in Experiment 1.

Prime recognition and priming effects. Across all subjects, we did not find any evidence for inverse priming. However, in Experiment 1 inverse priming effects seemed to be limited to those participants being unable to consciously perceive the prime. Thus, we examined whether priming effects on central targets again depended on prime visibility and whether inverse priming was present within those subjects showing poor prime recognition. Whereas RT-priming effects averaged across both sessions did not show any correlation with prime recognition performance $(\mathrm{r}=-.15, \mathrm{p}=.60)$, a positive correlation was observed for priming effects on error rates $(r=.69, \mathrm{p}<.01)$. However, when comparing priming effects between those subjects showing poor and good prime recognition performance no differences were obtained neither for RTs and the stricter, $\mathrm{F}(1,12)<1$, or the more liberal criterion, $\mathrm{F}(1,12)<$ 1 , nor on error rates for the stricter, $\mathrm{F}(1,12)=2.0, \mathrm{p}=.19$, or the more liberal criterion, $\mathrm{F}(1,12)<1$. However, the good performance group was rather small (four participants for the stricter criterion and only a single one for the more liberal criterion) leading to unequal group sizes which undermines statistical power. Therefore, we tested whether inverse priming could be found within the poor prime recognition group. However, inverse priming on RTs was absent independent of which criterion or session was considered (all $t<.90, \mathrm{p}>.38$ ). The same holds true for error rate data (all $\mathrm{t}<.97, \mathrm{p}>.35$ ).

\section{Discussion}

To our surprise, we did not observe any priming effects neither for central nor for lateral targets even when only looking at those subjects showing subliminal prime perception. In contrast to Experiment 1 which used double arrows as imperative stimuli, subjects seemed to be much slower classifying the arrow patterns used in Experiment 2 which were ambiguously assigned to the responses. To test this observation, data from Experiment 1 and 2 were subjected to a mixed ANOVA with the factors Congruency, Target Type and Experiment. The performance advantage for responses to the lateral targets (which were identical for both experiments) was larger in Experiment $2(131 \mathrm{~ms})$ compared to Experiment $1(15 \mathrm{~ms})$ as indexed by the interaction of Target Type and Experiment, $F(1,42)=59.7, p<.0001$. Apart from the response compatibility of double arrows with responses, another obvious difference 
to the arrow patterns of Experiment 2 is that double arrows constitute a natural object to which participants should have been exposed before. Therefore, we intended to replicate Experiment 2 with symbolic, more object-like stimuli.

\section{Experiment 3}

In Experiment 2 we did not obtain inverse priming effects with the arrow pattern stimuli. This might be due to the complex nature of the stimuli used. Therefore, Experiment 3 was designed to assess inverse priming effects with simple geometrical forms, namely a diamond and a cross, as prime and central target stimuli which should be easier accessible and therefore processed more rapidly.

Method

Participants. 14 new students from the University of Göttingen participated in Experiment 3 (3 males, mean age 24.5 years, std 3.1 years, range 20-33 years). All but one were righthanded by self-report, and all of them had normal or corrected-to-normal vision. Each participant received $24.50 €$ for participation in the three sessions of the experiment.

Task, Stimuli, Procedure, and Design. Experiment 3 differed from Experiment 2 only with respect to the prime and target stimuli used. An ' $x$ '-shaped target required left-hand responses and a diamond-shaped target right-hand responses. Both stimuli subtended $0.7^{\circ} \times 0.7^{\circ}$ of visual angle. Because perceptual similarity among primes and lateral targets is crucial here, the lateral '+'-signs were also replaced by a red lateral dot of $0.1^{\circ} \mathrm{x} 0.1^{\circ}$ of visual angle to increase dissimilarity to the new prime stimuli. Mask stimuli were left unchanged. Again, two Choice RT sessions were acquired.

Results

$R T$. Responses to lateral targets were faster $(377 \mathrm{~ms})$ than to central targets $(461 \mathrm{~ms})$ as indexed by the main effect of Target Type, $F(1,13)=71.4, p<.0001$ (see Figure 6). Overall RT differed across the two sessions, $\mathrm{F}(1,13)=37.4, \mathrm{p}<.0001$ : responses were slower in the 
first (439 ms) as compared to the second session (400 ms). Congruency did not affect response times and no other effects were significant.

Accuracy data. On average responses were incorrect on $2.1 \%$ of all trials. Target Type influenced error rates, $\mathrm{F}(1,13)=42.3$, $\mathrm{p}<.0001$, with fewer errors for lateral $(0.3 \%)$ as compared to central targets $(4.0 \%)$. The main effect of Congruency was not significant, $F(1,13)<1$. No further effects reached significance.

Please insert Figure 6 about here

Prime recognition. Prime recognition performance differed across the two Target Types, $\mathrm{t}(13)$ $=-3.7, \mathrm{p}<.01$. Prime visibility was higher for lateral $(65.2 \%)$ as compared to central targets $(69.9 \%)$. With both types of targets, performance was significantly above chance level, $\mathrm{t}(13)$ $>2.9, \mathrm{p}<.05$. Prime recognition ranged from $46.5 \%$ to $95.8 \%$ for central targets and from $48.2 \%$ to $98.2 \%$ for lateral targets. Again, we checked whether priming effects for central targets depended on visibility.

Prime recognition and priming effects for central targets. Prime recognition and priming effects were correlated with respect to RTs, $r=.67, \mathrm{p}<.01$, but not with respect to error rates, $\mathrm{r}=.30, \mathrm{p}=.28$. However, priming effects on RTs did not differ between those participants with good versus poor prime recognition performance when the strict criterion was adopted, $\mathrm{F}(1,12)=1.1, \mathrm{p}=.31$. In contrast, for the more liberal criterion priming effects differed among the two groups, $\mathrm{F}(1,12)=10.8, \mathrm{p}<.01$. Effects did not significantly deviate from zero for those subjects being unable to perceive the prime, $-7 \mathrm{~ms}, \mathrm{t}(8)=-1.3, \mathrm{p}=.24$, and positive for the remaining participants, $+20 \mathrm{~ms}, \mathrm{t}(4)=3.8, \mathrm{p}<.05$. Priming effects on error rates were indistinguishable across the two groups irrespective of whether the strict, $F(1,12)<1$, or the more liberal criterion, $\mathrm{F}(1,12)=4.3, \mathrm{p}=.06$, was considered. 


\section{Discussion}

Again, we failed to find inverse priming effects. Even when restricting the analysis to those subjects being unable to perceive the prime we did not observe any evidence for inverse priming. At first glance, results from Experiment 2 and 3 might suggest that inverse priming with irrelevant masks only exists for arrow-like prime and target stimuli. Evidence for this view has been presented before by Jaśkowski and Ślósarek (2007) who found inverse priming effects only for double arrows or arrow-like rectangular brackets but not for non-arrow stimuli like diamonds and squares. Such arbitrarily mapped stimuli only yielded inverse priming with a relevant mask consisting in the superposition of both stimuli. With such relevant masks, presentation of the mask seems to be equivalent to the occurrence of a second opposite prime. Thus, inverse effects are simply induced by this perceptual interaction of prime and mask (Lleras, \& Enns, 2004; Verleger, Jaśkowski, Aydemir, van der Lubbe, \& Groen, 2004). The use of non-arrow stimuli in the study of inverse priming in combination with irrelevant masks is rather rare. Verleger, Görgen and Jaśkowski (2005) found inverse priming with pairs of parallel horizontal lines intersected by a vertical line either on the left or the right side of these lines. However, these stimuli also resemble arrows insofar as they point to either side. To the best of our knowledge almost all other demonstrations of inverse priming with non-arrow stimuli employed relevant (mostly metacontrast) masks (Eimer, 1999; Jaśkowski, \& Ślósarek, 2007; Kiesel, Berner, \& Kunde, 2008; Mattler, 2006, 2007; Krüger, Klapötke, \& Mattler, 2010). However, two exceptions are found in the literature that deserve further consideration: First, Schlaghecken and colleagues employed so-called Landolt ring stimuli - an annulus with a gap on either the left-hand or right-hand side (similar to the letter 'C'; Schlaghecken, Rowley, Sembi, Simmons, \& Whitcomb, 2007). These stimuli were masked by an array of circles such that presentation of the mask was not equivalent to adding the prime-contrary stimulus alternative. However, evidence seemed to be rather fragile because effects were absent with the typical prime duration of about or less than $30 \mathrm{~ms}$. Only increasing prime duration to $50 \mathrm{~ms}$ let to a stable effect. Second, Sumner repeatedly demonstrated reliable inverse priming with pairs of either horizontally or vertically aligned bars which were masked by random line masks typically sparing out orientations close to the prime orientations (Boy, Clarke, \& Sumner, 2008; Boy, \& Sumner, 2010; Sumner, 2008). Thus, such masks could be readily held for irrelevant masks. Additionally, these effects seemed to be particularly robust insofar as they were found in almost every single subject (Boy, \& Sumner, 2010). Therefore, we next tried to replicate these finding and also considered to what degree effects translate to perceptually dissimilar targets. 


\section{Experiment 4a}

We intended to replicate inverse priming effects with bar pairs and to further assess whether effects propagate to perceptually dissimilar lateral targets. To this end, we rebuilt primes and central targets according to Sumner (2008). To ensure that the mask is free of task-relevant features, we also created a new mask which was composed of oblique lines only (see Figure $1)$.

\section{Method}

Participants. 14 new students from the University of Göttingen participated in Experiment 4a (7 males, mean age 23.3 years, std 2.7 years, range 20-30 years). All but one were righthanded by self-report, and all of them had normal or corrected-to-normal vision. Each participant received $24.50 €$ for participation in the three sessions of the experiment.

Task, Stimuli, Procedure, Design. Experiment 4 replicated Experiment 3 using different stimuli. Central targets were bar pairs. When the bar pair was horizontally aligned a left-hand response was required and a right-hand response for vertically aligned bar pairs. Both bar pairs subtended $1^{\circ}$ x $1^{\circ}$ of visual angle. Red dots served again as lateral targets. Masks now comprised only oblique lines of random orientations sparing out those orientations closer than $5^{\circ}$ to those of the targets.

Results

$R T$. Response times were not affected by Congruency, $\mathrm{F}(1,13)<1$. Target Type influenced RTs, $\mathrm{F}(1,13)=60.5, \mathrm{p}<.0001$, with faster responses to lateral $(382 \mathrm{~ms})$ as compared to central targets (481 ms). Also, response speed increased across Sessions, $F(1,13)=69.3, p<$ .0001 . Average RTs in the first session $(457 \mathrm{~ms})$ were larger as compared to the second session (406 ms). No further effects were significant.

Accuracy data. The overall error rate was $3.2 \%$. Target Type influenced the error rate, $\mathrm{F}(1,13)=108.1, \mathrm{p}<.0001$, more errors were committed in response to central $(6.2 \%)$ as compared to lateral targets $(0.1 \%)$. The main effect of Congruency failed to reach significance, $F(1,13)=2.6, p=.13$. No other effects were significant. 
Please insert Figure 7 about here

Prime recognition. Recognition performance did not differ for central and lateral targets, $\mathrm{t}(13)$ $=-.4, \mathrm{p}=.66$. On average, $63.3 \%$ of all primes were correctly reported which is significantly better than chance level, $\mathrm{t}(13)=16.7, \mathrm{p}<.0001$. Recognition performance ranged across subjects from $48.6 \%$ to $93.4 \%$.

Prime recognition and priming effects. Priming effects on RTs were again correlated with prime visibility, $\mathrm{r}=.57, \mathrm{p}<.05$, but effects on error rates did not show such a correlation, $\mathrm{r}=$ $.27, \mathrm{p}=.34$. However, according to the stricter criterion, $\mathrm{F}(1,12)<1$, as well as to the more liberal one, $\mathrm{F}(1,12)<1$, no difference on RT-priming effects was observed between those subjects showing subliminal and those showing supraliminal prime perception. In contrast, priming effects on error rates differed between the two groups divided according to the stricter criterion and this effect of visibility differed between the two sessions as indexed by the interaction of Session, Visibility Group and Congruency, $F(1,12)=5.2, p<.05$. While for those subjects with subliminal prime perception a trend towards inverse priming was observed during the first, $-4.5 \%, \mathrm{t}(3)=2.9, \mathrm{p}=.06$, and the second session, $-3.3 \%, \mathrm{t}(3)=2.5, \mathrm{p}=.09$, no priming effects were found for those with supraliminal prime perception neither in the first, $1.1 \%, \mathrm{t}(9)=-.3, \mathrm{p}=.75$, nor in the second session, $-1.6 \%, \mathrm{t}(9)=1.4, \mathrm{p}=.20$. When considering the more liberal criterion, no such group differences were found, $F(1,12)<1$, and no interaction of Session, Group and Congruency, $\mathrm{F}(1,12)<1$.

\section{Discussion}

In Experiment 4 we intended to replicate inverse priming effects with stimuli which proofed to produce inverse priming in several prior studies (Sumner, 2008; Boy, et al., 2008; 2010). Notably, RT performance was not affected at all by prime-target congruency. We only found a very weak, insignificant tendency towards inverse priming on error rates for central targets among those subjects with subliminal prime perception. Notably, the complete absence of RT effects in our experiment is not due to better prime visibility compared to Sumner (2008) who 
excluded two subjects with almost perfect prime recognition because when only those subjects with poor prime recognition were considered priming effects were still absent. There are some further differences to Sumner (2008) which could potentially account for the absence of inverse priming in the current experiment: first, in Sumner (2008) the line orientations within the mask were taken from a more discrete orientation spectrum only including orientations deviating from the prime orientation from $22.5^{\circ}$ to $157.5^{\circ}$ (his Mask B) in steps of $22.5^{\circ}$. In contrast, our mask was composed of line elements whose orientations ranged from $5^{\circ}$ to $175^{\circ}$ in steps of $1^{\circ}$. Second, each participant experienced two slightly different types of masks in Sumner (2008). One mask included prime orientations (Mask A) and another one sparing them out (Mask B). Both masks yielded inverse priming effects of comparable magnitude. Third, participants performed 400 trials in contrast to 576 in the current experiment. Fourth, in Sumner (2008) RTs shorter than $200 \mathrm{~ms}$ and above $800 \mathrm{~ms}$ were excluded from data analysis. Post-error trials were not excluded. Fifth, intertrial intervals were of a constant duration of $1000 \mathrm{~ms}$ and thus shorter as the average interval in the current experiment. Sixth, Sumner's experiment (2008) did not include lateral targets.

We reanalyzed our data to rule out that the absence of inverse priming effects in our experiment was due to the greater number of trials or the different way to summarize RT data. To this end, we adopted the exclusion criteria of Sumner (2008) and restricted the analysis to the first 400 trials of each session. However, even with these adaptations priming effects did not differ from zero, neither in the first, $+11 \mathrm{~ms}, \mathrm{t}(13)=-1.7, \mathrm{p}=.11$, nor for the second session, $-1 \mathrm{~ms}, \mathrm{t}(13)=.1, \mathrm{p}=.95$. Beyond this, the analysis revealed that participants responded faster in Sumner's experiment (525 ms compared to about $410 \mathrm{~ms}$, estimated based on Figure 2, Sumner (2008)) but error rates were a bit lower (7.5\% compared to about $5 \%$ ). This performance difference could be due to the inclusion of lateral targets in our experiment. Performance might suffer in the light of the more heterogeneous target set. Apart from global performance, this might have also affected priming effects. The fact that primes appeared as targets on only $50 \%$ of all trials might have reduced the prime's impact on target processing such that priming effects disappeared. This interpretation is supported by the fact that inverse priming effects completely disappear when primes and target sets are entirely distinct: Eimer and Schlaghecken (1998, Exp. 1b) failed to find inverse priming for arrow primes combined with letter targets (LL, RR). Therefore, we replicated Experiment 4a leaving out lateral target stimuli. 


\section{Experiment 4b}

Experiment $4 \mathrm{~b}$ was an exact replication of Experiment $4 \mathrm{a}$ apart from the fact that only central bar pairs were presented as target stimuli, thereby reducing the stimulus set to only two imperative stimuli.

Method

Participants. 14 new students from the University of Göttingen participated in Experiment 4b (9 males, mean age 25.7 years, std 3.0 years, range 21-33 years). All but two were righthanded by self-report, and all of them had normal or corrected-to-normal vision. Each participant received $24.50 €$ for participation in the three sessions of the experiment.

Task, Stimuli, Procedure, Design. Apart from the exclusion of lateral targets, all other aspects of Experiment $4 \mathrm{~b}$ were identical to Experiment $4 \mathrm{a}$.

Results

$R T$. RTs changed with training as indexed by the main effect of Session, $\mathrm{F}(1,13)=5.3$, $\mathrm{p}<$ .05 . RTs were shorter in the second $(425 \mathrm{~ms})$ as compared to the first session (440 ms). While the main effect of Congruency failed to reach significance, $F(1,13)=3.8, p=.07$, the interaction of Congruency and Session was significant, $F(1,13)=12.9, p<.01$. Priming effects were absent in the first session, $-2 \mathrm{~ms}, \mathrm{t}(13)=.6, \mathrm{p}=.56$, but present in the second session, $-13 \mathrm{~ms}, \mathrm{t}(13)=3.2, \mathrm{p}<.01$.

Accuracy data. No effect reached significance.

Prime recognition. On average, $54.1 \%$ of all primes were correctly reported which does not significantly differ from chance level, $\mathrm{t}(13)=1.7, \mathrm{p}=.11$. Prime recognition performance ranged from $45.7 \%$ to $81.4 \%$.

Prime recognition and priming effects. Prime visibility and priming effects were uncorrelated with respect to $\mathrm{RT}$ data, $\mathrm{r}=.38, \mathrm{p}=.17$, and error rates, $\mathrm{r}=.31, \mathrm{p}=.28$. Considering the strict criterion, priming effects on RTs differed between the two visibility groups, $\mathrm{F}(1,12)=9.5$, $\mathrm{p}$ .01. Those subjects with subliminal prime perception showed inverse priming effects, $-12 \mathrm{~ms}$, $\mathrm{t}(9)=-2.8, \mathrm{p}<.05$ whereas those with supraliminal prime perception did not show any 
priming effect, $+9 \mathrm{~ms}, \mathrm{t}(3)=-1.7, \mathrm{p}=.19$. The same interaction of visibility group and Congruency reached significance for error rates, $\mathrm{F}(1,12)=5.3, \mathrm{p}<.05$. However, neither those with subliminal prime perception, $-.8 \%, \mathrm{t}(9)=1.8, \mathrm{p}=.12$, nor the remaining participants showed any priming effect, $1.6 \%, \mathrm{t}(3)=-1.9, \mathrm{p}=.16$. In contrast, when the more liberal criterion was considered, no group differences were found with respect to RT data, $\mathrm{F}(1,12)=1.5, \mathrm{p}=.24$, and to error rates, $\mathrm{F}(1,12)<1$.

\section{Discussion}

While in Experiment 4a we completely failed to observe inverse priming with bar pairs as prime and target stimuli (disregarding an insignificant trend for the error rate data among those subjects with subliminal prime perception) we found evidence when we omitted lateral targets in Experiment $4 \mathrm{~b}$. Augmenting the target set by lateral stimuli might diminish the capability of individual items to trigger processes leading to inverse priming. When the entire sample of participants was considered, inverse priming was restricted to the second session. The importance of training for inverse priming to occur has been repeatedly shown (Klapp, \& Hinkley, 2002; Przekoracka-Krawczyk, \& Jaśkowski, 2007; Schlaghecken, Blagrove, \& Maylor, 2007). This seems to be at odds with recent data by Boy and Sumner (2010) who reported inverse priming for almost every single subject after only 20 practice trials with the same stimulus material. Apart from practice, however, prime visibility also influenced the direction of priming effects. In the current study, average prime recognition performance was comparable to Boy and Sumner (2010). However, the procedure of estimating prime visibility differs. In contrast to Boy and Sumner (2010), subjects performed a separate prime recognition session during which they received feedback. Additionally, Boy and Sumner (2010) omitted targets during the prime recognition part. Therefore, it seems difficult to directly compare results but it seems plausible that Boy and Sumner (2010) achieved better masking which led to more stable inverse priming effects right from the beginning.

Across experiments, we found an astonishing variability of inverse priming effects with changing stimulus material. While double arrows produced reliable inverse priming effects, arbitrarily mapped stimuli like the arrow patterns in Experiment 2 or geometrical shapes in Experiment 3 did not lead to inverse priming which is in accord with previous studies on inverse priming with non-compatible stimulus material and irrelevant masks (Jaśkowski \& Ślósarek , 2007; Kiesel, Berner, \& Kunde, 2008, Klauer, \& Dittrich, 2010, Exp.3 Single- 
Family Group). At first glance, this might suggest that inverse priming is limited to arrow-like stimuli being compatible to the response set (Jaśkowski \& Ślósarek , 2007). However, less pronounced inverse priming effects were also obtained with bar pair stimuli that are not spatially compatible to the response set. However, the delayed onset of inverse priming compared to arrow stimuli suggests a different mechanism producing inverse priming. In contrast, to the stimuli from in Experiment 2 and 3 bar pairs are rather similar to the mask elements which consist of lines of similar width and length only differing with respect to their orientation. Therefore, it seems possible that inverse priming is induced by the mask: the rapid serial presentation of prime and mask might facilitate processing of those line elements within the mask whose orientations differs most from the prime's orientation facilitating processing of incongruent targets (Lleras, \& Enns, 2004). Thus, the current masks even though sparing out target orientations could be interpreted as relevant masks. With such relevant masks, perceptual learning seems to facilitate recognition of task-relevant mask features (Przekoracka-Krawczyk, \& Jaśkowski, 2007; Klapp, \& Hinkley, 2002) which would explain why the onset of inverse priming effects was delayed. Furthermore, PrzekorackaKrawczyk and Jaśkowski (2007) found that inverse priming effects occurred earlier for less complex masks suggesting that mask-induced priming occurs earlier when task-relevant features are easier extractable from such less complex masks. Maybe, Boy and colleagues (2008) whose masks were composed of lines from less different orientations could also be considered less complex compared to our masks which might account for why they observed inverse priming effects from almost right the beginning of the experiment. However, we have to note that Sumner (2008) found equal priming effects for a mask containing the target orientations and one leaving them out casting doubts on the interpretation of bar pair priming as a phenomenon resulting from perceptual interactions of primes and masks because effects are expected to be larger when the target orientations themselves are part of the mask.

In contrast to the bar pair stimuli, mask-induced priming cannot account for inverse priming with double arrows and the random line masks employed because they were entirely free of arrow-like features. As discussed in Experiment 3, double arrows or arrow-like brackets account for the vast majority of inverse priming effects. Therefore, a special status of arrows for inverse priming has been suggested (Jaśkowski \& Ślósarek, 2007; Verleger, Jaśkowski, Aydemir, van der Lubbe, \& Groen, 2004; Verleger, Görgen, \& Jaśkowski; 2005) which might be either due to preexisting learned directional associations or to their response compatibility. This question can be addressed by reverting the natural stimulus-response mapping for double arrows (i.e. left response to a rightward pointing arrow and vice versa). Such a reversal of 
stimulus-response pairings impairs overall performance but does not affect inverse priming (Jaśkowski \& Ślósarek , 2007; O’Connor, \& Neill, 2011) leading to the conclusion that response compatibility is crucial for inverse priming to occur with irrelevant masks. Further support casting doubt on a special status of arrows due to learning comes from a recent demonstration of inverse priming with non-arrow stimuli (Schlaghecken, et al. 2007). In this experiment, circle stimuli with a missing section on either the left-hand or right-hand side served as primes and targets. These stimuli do not represent overlearned symbols for 'left' and 'right' like arrows but they are still response compatible.

That processing of response compatible stimuli is qualitatively different from processing of non-compatible stimuli is a central assumption of dual-route models of perceptuo-motor translation (Hommel, 2000). According to the dimensional overlap model (Kornblum, Hasbroucq, \& Osman, 1990), automatic and intentional response activation compete against each other whenever stimuli and responses have features in common like the spatial alignment of double arrows and the spatial alignment of response buttons in our setting. This situation is called dimensional overlap. A rapid, automatic translation of perceptual input is accompanied by a second controlled translation route which ensures that the automatic activations only results in a response when it matches the current task demands. Inverse priming with irrelevant masks might operate only on the automatic route and therefore, dimensional overlap is prerequisite for inverse priming to occur. Therefore, we tried to find inverse priming with response compatible stimuli that do not resemble double arrows.

\section{Experiment 5}

In Experiment 5, lateral targets were again intermixed with central targets. Primes and central targets were a combination of a horizontal bar and a dot forming a "horizontal exclamation mark". To improve masking, the exact horizontal and vertical position varied randomly from trial to trial below the mask. While the dot and the bar were always presented at the same vertical position, the horizontal spacing of both elements varied independently.

\section{Method}

Participants. 14 new students from the University of Göttingen participated in Experiment 5 (5 males, mean age 24.2 years, std 2.8 years, range 20-29 years). All but three were right- 
handed by self-report, and all of them had normal or corrected-to-normal vision. Each participant received $24.50 €$ for participation in the three sessions of the experiment.

Task, Stimuli, Procedure, Design. Compared to Experiment 4, we again employed '+'-signs as lateral targets which seemed to be more distinct than red dots to the central target stimuli. Again identical to Experiment 3, the orthogonal mask was employed. The vertical position of the prime and target stimuli varied $0.2^{\circ}$ of visual angle around fixation. The horizontal position was independently determined for the dot and bar element within the stimuli. It ranged from $0.1^{\circ}$ to $0.25^{\circ}$ of visual angle deviation from fixation. The size of the dot element was around $0.05^{\circ} \times 0.05^{\circ}$. The horizontal extension of the bar element was twice of that of the dot. In contrast to previous experiments, stimulus-response mappings were counterbalanced across participants. Half of them had to respond with the left index finger when the dot was left and with the right index finger when the dot was right. The remaining participants responded to the position of the bar with the index finger of the corresponding side.

Results

$R T$. Congruency did not affect response times, $\mathrm{F}(1,13)=2.4, \mathrm{p}=.15$. Response latencies changed across the sessions, $\mathrm{F}(1,13)=16.4, \mathrm{p}<.01$. Responses were faster during the second (374 ms) compared to the first session (406 ms). Target Type also influenced response speed, $\mathrm{F}(1,13)=66.1, \mathrm{p}<.0001$. Responses to lateral targets $(362 \mathrm{~ms})$ were faster compared to central targets $(418 \mathrm{~ms})$. No other effects reached significance.

Accuracy data. The overall error rate was $3.6 \%$. It was affected by Congruency, $\mathrm{F}(1,13)=$ $4.7, \mathrm{p}<.05$. The error rate was lower on congruent $(2.9 \%)$ compared with incongruent $(4.2$ $\%)$ trials which corresponds to a positive priming effect. In addition, Target Type also influenced the error rate, $\mathrm{F}(1,13)=32.0, \mathrm{p}<.0001$, with fewer errors for lateral $(0.1 \%)$ as opposed to central $(6.2 \%)$ targets. No further effects were significant.

Please insert Figure 8 about here 
Prime recognition. Prime recognition performance did not differ between central and lateral targets, $\mathrm{t}(13)=-1.9, \mathrm{p}=.09$. Primes were correctly reported on $54.4 \%$ of all trials which does not significantly differ from chance level, $t(13)=1.5, p=.16$. Individual performance ranged from $46.0 \%$ to $83.5 \%$.

Prime recognition and priming effects on central targets. Priming effects on RTs were correlated with prime recognition performance, $\mathrm{r}=.56, \mathrm{p}<.05$, but not priming effects on error rates, $\mathrm{r}=.23, \mathrm{p}=.42$. Considering the strict criterion, priming effects on $\mathrm{RTs}, \mathrm{F}(1,12)=$ $3.8, \mathrm{p}=.07$, and error rates, $\mathrm{F}(1,12)=3.6, \mathrm{p}=.08$, did not differ among the two visibility groups. The same holds for the more liberal criterion with respect to both, RTs, $\mathrm{F}(1,12)=4.2$, $\mathrm{p}=.06$, and error rates, $\mathrm{F}(1,12)=1.4, \mathrm{p}=.26$.

\section{Discussion}

Again, we did not obtain inverse priming effects with the current stimulus material. The initial idea was to develop stimuli that are compatible to the spatial alignment of the responses but that are not associated with the abstract concepts of 'left' and 'right' like arrows. The horizontal exclamation marks seemed to fulfill these requirements. The question, however, is what aspects of a given stimulus render it response compatible. It seems plausible that response activation processes are directly driven by early visual analyses of the global asymmetry of the current perceptual input. If the total amount of asymmetrical features along the spatial dimension of the response buttons within a given stimulus determines its response compatibility, double arrows would be much more compatible. The exclamation marks possess a distracter on the opposite site of its critical feature that reduces the total asymmetry which might preclude automatic response activation. Within the double arrows none of the elements has a counterpart on the opposite site. This might guarantee automatic processing and direct activation of a motor response which is also reflected in faster responses to the central double arrows compared to lateral stimuli, $\mathrm{F}(1,42)=26.1, \mathrm{p}<.0001$. While responses to double arrows were only by $15 \mathrm{~ms}$ slower compared to lateral targets, responses to the exclamation mark stimuli were about $56 \mathrm{~ms}$ delayed compared to the same lateral targets. 
To sum up, even though the present stimuli possess some degree of response compatibility it might not be enough to trigger an automatic response activation and instead stimuli are subjected to a controlled response selection process.

\section{Experiment 6}

In a new attempt, we created new stimuli increasing the asymmetry in order to induce automatic processing of these stimuli. To this end, we employed small crosses shifted either to the left or right from fixation (below the mask) and omitted the distracting features on the contralateral side.

\section{Method}

Participants. 14 new students from the University of Göttingen participated in Experiment 6 (4 males, mean age 25.1 years, std 2.2 years, range 21-30 years). All of them were righthanded by self-report, and all of them had normal or corrected-to-normal vision. Each participant received $24.50 €$ for participation in the three sessions of the experiment.

Task, Stimuli, Procedure, Design. Apart from primes and central targets, Experiment 6 was identical to Experiment 5. Now, we employed small crosses of $0.2^{\circ} \times 0.2^{\circ}$ of visual angle which were presented at a random location below the mask ranging from $0.2^{\circ}$ to $0.3^{\circ}$ of horizontal deviation from fixation and less than $0.3^{\circ}$ deviation on the vertical axis in order to optimize masking efficiency.

\section{Results}

$R T$. Results are illustrated in Figure 9. Response times were affected by Congruency, $\mathrm{F}(1,14)$ $=9.1, \mathrm{p}<.01$. Responses on congruent trials $(310 \mathrm{~ms})$ were faster compared to incongruent trials $(320 \mathrm{~ms}$ ) which corresponds to a positive priming effect. Response speed changed across the sessions, $\mathrm{F}(1,13)=10.5, \mathrm{p}<.01$. Latencies were shorter in the second $(309 \mathrm{~ms})$ than in the first session (322 ms). Target Type also affect RTs, $F(1,13)=68.4, p<.0001$. Responses were faster to lateral $(300 \mathrm{~ms})$ as compared to central targets $(331 \mathrm{~ms})$. This advantage for central targets differed between the two sessions as indexed by the interaction 
of Session and Target Type, $\mathrm{F}(1,13)=12.5, \mathrm{p}<.01$. During the first session the advantage was larger (34 ms) compared to the second session $(29 \mathrm{~ms})$. No further results reached significance.

Accuracy data. Mean error rate was $2.7 \%$. It was not affected by Congruency, $\mathrm{F}(1,13)=4.2$, $\mathrm{p}=.06$, but by Target Type, $\mathrm{F}(1,13)=87.8, \mathrm{p}<.0001$. Subjects made more errors for central $(4.6 \%)$ than for lateral targets $(0.8 \%)$. Priming effects differed for central and lateral targets, $\mathrm{F}(1,13)=5.3, \mathrm{p}<.05$. While effects were positive for central targets, $1.8 \%, \mathrm{t}(13)=-2.6, \mathrm{p}<$ .05 , no priming effects were observed for lateral targets, $0.3 \%, \mathrm{t}(13)=-.6, \mathrm{p}=.58$. No other effects were significant.

Please insert Figure 9 about here

Prime recognition. Prime recognition performance was affected by Target Type, $\mathrm{t}(13)=-2.2$, $\mathrm{p}<.05$. Performance was better for lateral $(64.2 \%)$ compared to central targets $(60.9 \%)$ which differed from chance level, both $\mathrm{t}(13)>2.8, \mathrm{p}<.05$. Performance ranged from $47.2 \%$ to $94.1 \%$ for central and from $42.7 \%$ to $90.3 \%$ for lateral targets.

Prime recognition and priming effects for central targets. Priming effects on $\mathrm{RTs}, \mathrm{r}=-.01, \mathrm{p}$ $=.96$, and error rates, $\mathrm{r}=-.08, \mathrm{p}=.77$, were not correlated with prime recognition performance. With respect to the stricter criterion, priming effects were indifferent among the two visibility groups for RTs, $F(1,12)<1$ and error rates, $F(1,12)<1$. However, differences on error rate priming effects between the groups differed across the two sessions, Congruency x Group x Session: $F(1,12)=5.1, p<.05$. However, inverse priming was absent in all groups and sessions. Also for the more liberal criterion, priming effects were the same for both visibility groups with respect to both, $\mathrm{RTs}, \mathrm{F}(1,12)<1$, and error rates, $\mathrm{F}(1,12)<1$. 


\section{Discussion}

Inverse priming was again absent with the slightly eccentric cross stimuli. In contrast to double arrows, the current task required reporting the position of the target stimulus relative to fixation instead of the identification of an object. However, the sudden onset of an eccentric stimulus shifts spatial attention (e.g. Eriksen, \& Hoffman, 1972; Todd, \& van Gelder, 1979). After such a shift the spatial reference frame is centered on the object thereby potentially eliminating the asymmetry within the stimulus because the cross object itself is symmetric. Therefore, inverse priming might only work with response compatible objects which are presented centrally thus not eliciting automatic shifts of attention. Then, the task involves identification of that object rather than the position where it is localized. Therefore, we made another attempt and created complex object-like stimuli which were presented centrally.

\section{Experiment 7}

In Experiment 7, we intended to use stimuli that are on the one hand as asymmetrical as possible but that comprise a single centrally presented object. Therefore, we used barbell-like stimuli which were built by the small cross on the one side and a larger diamond on the other side both connected by a horizontal line (see Figure 1). The elements on both sides did not overlap when overlaid, thus allowing for maximal left-right asymmetry.

\section{Method}

Participants. 14 new students from the University of Göttingen participated in Experiment 7 (4 males, mean age 22.8 years, std 3.2 years, range 19-30 years). All of them were righthanded by self-report, and all of them had normal or corrected-to-normal vision. Each participant received $24.50 €$ for participation in the three sessions of the experiment.

Task, Stimuli, Procedure, Design. The barbell-like stimuli were built based on the cross stimuli employed in Experiment 6 and the diamond from Experiment 3. The size of these elements was left unchanged. The horizontal separation of the centre of both elements was $0.8^{\circ}$ of visual angle. Stimulus-response mappings were balanced across participants. Thus, half of the participants had to press the button spatially corresponding to the side of the cross, the remaining participants to the diamond. Stimuli were introduced to the subject as complex objects that had to identified in order to discourage them from responding to the position of a single feature within the object. 
Results

$R T$. Response latencies were influenced by Congruency, $\mathrm{F}(1,13)=5.1, \mathrm{p}<.05$. Overall responses were faster on congruent $(352 \mathrm{~ms})$ as compared to incongruent primes (358 $\mathrm{ms})$ corresponding to a positive priming effects. However, priming effects differed between the two sessions, $F(1,13)=8.9, p<.05$. While priming effects were positive during the first session, $9 \mathrm{~ms}, \mathrm{t}(13)=-3.7, \mathrm{p}<.01$, no effect of the prime was found in the second session, 2 $\mathrm{ms}, \mathrm{t}(13)=.6, \mathrm{p}=.55$. Additionally, response latencies changed with Session, $\mathrm{F}(1,13)=33.9$, $\mathrm{p}<.0001$. On average, responses were given faster in the second $(341 \mathrm{~ms})$ as opposed to the first session (369 ms). Target Type also influenced RTs, F(1,13) = 51.4, p <.0001. Responses to lateral targets were faster $(335 \mathrm{~ms})$ than to central targets $(376 \mathrm{~ms})$. No other effects were significant.

Accuracy data. The mean error was $1.7 \%$. Target Type affected it, $\mathrm{F}(1,13)=43.0, \mathrm{p}<.0001$. Trials with central targets were more prone to errors $(3.2 \%)$ than lateral target trials $(0.2 \%)$. Congruency just missed significance, $\mathrm{F}(1,13)=4.1, \mathrm{p}=.06$, but it significantly interacted with Session, $\mathrm{F}(1,13)=7.2, \mathrm{p}=.02$, and the three-way interaction of Session, Target Type and Congruency also reached significance, $\mathrm{F}(1,13)=5.8, \mathrm{p}<.05$. Priming effects on lateral targets were indistinguishable, Session $x$ Congruency $F(1,13)<1$, between the first, $+0.2 \%$, $\mathrm{t}(13)=-1.2, \mathrm{p}=.26$, and the second session, $+0.2 \%, \mathrm{t}(13)=-1.3, \mathrm{p}=.21$. In contrast, for central targets priming effects differed across the two sessions, $\mathrm{F}(1,13)=12.3$, $\mathrm{p}<.01$. Effects were clearly positive in the first, $+2.2 \%, \mathrm{t}(13)=-2.7, \mathrm{p}<.05$, but absent in the second session, $+0.2, \mathrm{t}(13)=-1.2, \mathrm{p}=.26$. No other effects were significant.

Please insert Figure 10 about here

Prime recognition. Prime recognition performance did not differ between central and lateral targets, $\mathrm{t}(13)=-.9, \mathrm{p}=.38$. Average performance was $68.5 \%$ which was significantly above chance level, $\mathrm{t}(13)=3.6, \mathrm{p}<.01$. Individual performance ranged from $45.5 \%$ to $99.7 \%$ of correct prime reports. 
Prime recognition and priming effects for central targets. Prime recognition performance and priming effects on RTs were correlated, $\mathrm{r}=.60, \mathrm{p}<.05$, but not effects on error rates, $\mathrm{r}=.36$, $\mathrm{p}=.20$. According to the stricter criterion, RT priming effects were indifferent across the two groups, $\mathrm{F}(1,12)<1$. In contrast, when the more liberal criterion was adopted, priming effects differed, $F(1,12)=7.8, p<.05$. While those being unable to perceive the prime did not show any priming effect, $-3 \mathrm{~ms}, \mathrm{t}(7)=.6, \mathrm{p}=.52$, the others showed positive priming, $+15 \mathrm{~ms}, \mathrm{t}(5)$ $=-3.3, \mathrm{p}<.05$. With respect to error rates, priming effects were not different between visibility groups irrespective of which criterion had been chosen, $F(1,12)<1$ and $F(1,12)=$ $1.4, \mathrm{p}=.26$.

\section{Discussion}

Also the barbell-like objects did not produce inverse priming irrespective of prime visibility. In post-experimental interviews, 11 out of 14 subjects reported to have focused their attention during task performance on the critical element (the diamond for half of them and the cross for the remaining participants) instead of drawing attention to the entire object. Thus, the majority of subjects might have encoded the task as a position not an identification task. Thus, they responded to the position of "their" target feature within the stimuli (mappings were balanced across participants). Feature-based attention might have induced a similar shift as if only a single target element would have been presented eccentrically as in Experiment 6.

So far, we only found inverse priming effects for double arrows and bar pairs as central target stimuli. While we attributed the effect in case of double arrows to the automatic processing of these stimuli, the effect with bar pairs should be induced by the random line mask containing features which might be interpreted as task-relevant. However, it could also be that a factor common to both types of stimuli improves the circumstances for inverse priming to occur. In contrast to all other stimuli, both of these stimulus sets are built of parallel lines. Processing of parallel line elements might be facilitated. To rule out that inverse priming only results when parallel lines are contained in the stimuli we next employed single arrows as prime and target stimuli. 


\section{Experiment 8}

The only difference to Experiment 7 is that primes and central target stimuli were now single arrows which of course do not contain any parallel lines.

Method

Participants. 14 new students from the University of Göttingen participated in Experiment 5 (8 males, mean age 24.2 years, std 2.5 years, range 20-30 years). All but two were righthanded by self-report, and all of them had normal or corrected-to-normal vision. Each participant received $24.50 €$ for participation in the three sessions of the experiment.

Task, Stimuli, Procedure, Design. Single arrows of equal size as those contained in the double arrows were presented centrally. Response mappings were kept constant across participants and spatially compatible with the orientation of the arrows.

Results

$R T s$. Session affected RTs, $\mathrm{F}(1,13)=12.5, \mathrm{p}<.01$. Responses were slower in the first $(391$ ms) compared to the second session $(365 \mathrm{~ms})$. Target Type also influenced RTs, F(1,13) = $17.0, \mathrm{p}<.01$. RTs to central targets were slower $(388 \mathrm{~ms})$ than to lateral targets $(368 \mathrm{~ms})$. Both effects interacted, $F(1,13)=28.5$. The advantage for lateral targets increased with training. It was relatively small in the first $(11 \mathrm{~ms})$ compared to the second session $(28 \mathrm{~ms})$. Congruency did not affect RTs, $\mathrm{F}(1,13)<1$, even when differentiating for Target Types, Congruency x Target Type, $F(1,13)=1.4, p=.25$, or Session, Congruency x Session, $F(1,13)$ $=1.5, \mathrm{p}=.25$. No other effects reached significance.

Accuracy data. Overall, subjects responded incorrectly on $1.6 \%$ of all trials. Target Type influenced the error rate, $\mathrm{F}(1,13)=21.1, \mathrm{p}<.001$. Subjects made less errors for lateral $(0.5 \%)$ than for central targets $(2.8 \%)$. While the main effect of Congruency was far from being significant, $F(1,13)<1$, Congruency interacted with Session, $F(1,13)=5.8, p<.05$. Effects were slightly but insignificantly biased towards positive priming in the first session, $1.0 \%$, $\mathrm{t}(13)=1.5, \mathrm{p}=.15$, but absent in the second session, $-0.3 \%, \mathrm{t}(13)=.8, \mathrm{p}=.43$. No other effects were significant. 
Please insert Figure 11 about here

Prime Recognition. Recognition performance differed between the Target Types, $\mathrm{t}(13)=-2.9$, $\mathrm{p}<.05$. Average performance was $59.9 \%$ for central and $62.3 \%$ for lateral targets, both significantly above chance level, $\mathrm{t}(13)>2.4, \mathrm{p}<.05$. For central targets individual performance ranged from $45.8 \%$ to $95.5 \%$ and for lateral targets from $45.1 \%$ to $98.4 \%$.

Prime recognition and priming effects for central targets. Individual prime recognition performance showed a strong linear relationship to priming effects on RTs as indexed by the correlation coefficient, $\mathrm{r}=.88, \mathrm{p}<.0001$, but not to priming effects on error rates, $\mathrm{r}=.27, \mathrm{p}$ $=.35$. The following comparisons of priming effects between those showing subliminal versus supraliminal prime perception are illustrated in Figure 12. Irrespective of whether the stricter, $\mathrm{F}(1,12)=6.3, \mathrm{p}<.05$, or the more liberal criterion, $\mathrm{F}(1,12)=13.0, \mathrm{p}<.01$, was adopted to assign participants to prime visibility groups, priming effects differed for those being unable to perceive the prime and the remaining participants showing supraliminal prime perception. Irrespective of the criterion, subjects with subliminal prime perception showed inverse priming which however was significant only for the strict, $-22 \mathrm{~ms}, \mathrm{t}(5)=5.1, \mathrm{p}<.01$, but not for the liberal criterion, $-13 \mathrm{~ms}, \mathrm{t}(5)=1.4, \mathrm{p}=.21$. In contrast, those with supraliminal prime perception showed more variable priming effects statistically indistinguishable from zero, strict: $+13 \mathrm{~ms}, \mathrm{t}(7)=-1.1, \mathrm{p}=.31$, and liberal: $+39 \mathrm{~ms}, \mathrm{t}(7)=-2.3, \mathrm{p}=.15$. With respect to error rate effects, the results were less clear-cut. For the more liberal criterion, we did not observe any group differences with respect to priming effects, $F(1,12)=2.2, p=.16$. For the stricter criterion, however, changes of priming effects across sessions differed between the groups, Visibility Group x Congruency x Session: $\mathrm{F}(1,12)=13.6, \mathrm{p}<.01$. During the first session, both visibility groups did not show any priming effect, subliminal: $+2.4 \%, \mathrm{t}(5)=-.5$, $\mathrm{p}=.63$, supraliminal: $+0.8 \%, \mathrm{t}(7)=-1.1, \mathrm{p}=.30$. In contrast, in the second session priming effects reversed for those with subliminal prime perception, $-2.6 \%, \mathrm{t}(5)=3.2, \mathrm{p}<.05$, but not for the remaining subjects, $+1.0 \%, \mathrm{t}(7)=-1.1, \mathrm{p}=.29$. 


\section{Discussion}

When the entire sample of participants was considered inverse priming effects were absent. However, when we included prime recognition data into the consideration, we observed a tight link between priming effects and prime visibility. Inverse priming effects were limited to those subjects being unable to discriminate the prime while the other participant's responses were not affected at all by the prime. This relation of priming effects and prime recognition performance replicates findings from the double arrow data from Experiment 1 suggesting that single arrow effects are only reduced but do not qualitatively differ from double arrow effects. This interpretation is in line with other recent demonstrations of inverse priming with single arrow stimuli that were either entirely free of parallel lines (Jaśkowski, 2009; ) or all parallel elements were at least orthogonal to the spatial response alignment (Boy, Husain, \& Sumner, 2010). These demonstrations of inverse priming with single arrows contradict a crucial role of parallel elements for inverse priming according to the idea outlined in the introduction to this experiment.

Nevertheless, compared to double arrows inverse priming effects were clearly reduced as revealed by a mixed ANOVA upon the central target data from the first session of Experiment 1 and 9 including Congruency and Stimuli (double vs. single arrows) as factors. Priming effects differed between the experiments with respect to RTs, $\mathrm{F}(1,42)=4.9$, p $<.05(-23 \mathrm{~ms}$ vs. $-8 \mathrm{~ms})$, but the interaction just missed significance for error rates, $\mathrm{F}(1,42)=3.8, \mathrm{p}=.057$ $(-2.2 \%$ vs. $+1.5 \%)$. Because this initial session of both experiments was identical with respect to all aspects except for the prime and target stimuli used, the reduction of inverse priming is completely due using a single instead of a double arrow. Thus, the size of inverse priming seems to depend on the amount of discriminatory information available to the visual system. Similarly, peripheral presentation of primes also weakened their influence leading to smaller inverse priming effects (Schlaghecken, \& Eimer, 2000; Lingnau, \& Vorberg, 2005) 
Integrating findings from Experiment 1 to 8, we observed a surprising stimulus dependency of inverse priming effects. Evidence for inverse priming was unequivocal for double arrows, somewhat more ambiguous for single arrows and bar pair stimuli, but clearly absent for all other stimuli tested including stimulus material that is non-compatible to the response set (Experiment 2 and 3) and compatible stimuli (Experiment 5,6 and 7) which, however, might not have been holistically processed but instead required responding to the position of a certain target feature within a rather complex stimulus. We argued that inverse priming is only possible with response compatible stimuli that are processed as an object, like double arrows, the Landolt ring stimuli (Schlaghecken, et al., 2007), the rectangular brackets (Jaśkowski \& Ślósarek , 2007) or the intersected line stimuli (Verleger, et al., 2005). However, since inverse priming effects are known to depend on mask-target SOA (e.g. Mattler, 2007; Schlaghecken, \& Eimer, 2000), one could argue that inverse priming is principally also possible with other stimuli but that we just missed it because we concentrated on a single point in time which was chosen based on prior knowledge about the optimum for double arrows (Schlaghecken, \& Eimer, 2000). It is possible that the mask-induced reversal requires more time to reach its maximum for non-compatible stimuli. To rule out that inverse priming occurs relatively delayed with non-arrow stimuli we broadly varied the mask-target SOA using the geometrical shapes employed in Experiment 3 as target stimuli.

\section{Experiment 9}

Apart from investigating the influence of prolonging mask target SOAs, we pursued a second goal in Experiment 9. We tried to shed further light on the issue of whether the special role of arrows for inverse priming is due to overlearned associations to the concepts of "left" and "right" or to their spatial compatibility to the responses. The current evidence would favor the latter explanation (Jaśkowski \& Ślósarek , 2007; O’Connor, \& Neill, 2011; Schlaghecken, et al., 2007). However, if arrows nevertheless cause inverse priming because of their strong overlearned link to motor responses, then in principle other stimuli should have the same effect after extensive training. Learning of stimulus-response mappings has been considered as a crucial prerequisite for priming effects to occur (Boy, \& Sumner, 2010). Consequently, we tested this hypothesis and gave participants the opportunity to learn mappings across five sessions. If inverse priming depends on stable learned SR-links, one would expect that inverse priming effects grow with practice. 
Method

Participants. 14 new students from the University of Göttingen participated in Experiment 5 (9 males, mean age 23.4 years, std 4.5 years, range 18-38 years). All but two were righthanded by self-report, and all of them had normal or corrected-to-normal vision. Each participant received $42 €$ for participation in the six sessions of the experiment.

Tasks, Stimuli, Procedure. We chose the same stimuli used in Experiment 3. Lateral targets were omitted to ensure enough data in each experimental condition and to provide optimal conditions for inverse priming to occur (cf. results from Experiment 4a with lateral targets to Experiment $4 \mathrm{~b}$ without lateral targets). To study practice effects, subjects performed five Choice-RT sessions in advance to the prime recognition session. Mask-target SOA varied randomly from trial to trial between $0,100,150,200,250$ and $300 \mathrm{~ms}$. At the $0 \mathrm{~ms}$ SOA masks were not presented and thus positive priming was expected. All other aspects were identical to the previous experiments.

Design. We adopted a three-factorial 5x6x2 repeated measures design with the independent variables Session (1-5), SOA (0-300 ms) and Congruency. Since each session consisted of 576 trials we acquired 48 trials per condition.

Results

$R T$. Practice led to a decrease in response latencies, $\mathrm{F}(4,52)=20.7, \mathrm{p}<.0001$. Average RTs were 415, 391, 368, 360, $363 \mathrm{~ms}$ for the Sessions, respectively. SOA also affected RTs, $\mathrm{F}(5,65)=108.5, \mathrm{p}<.0001$. Apart from the 0 -ms SOA when no mask was presented, RTs decreased with increasing temporal separation of mask and target. The respective mean RTs were $408,415,381,362,356$, and $354 \mathrm{~ms}$ for the $0 \mathrm{~ms}$ to $300 \mathrm{~ms}$ SOA. This main effect of SOA was modulated by the Session, $\mathrm{F}(20,260)=6.3$, $\mathrm{p}<.05$. More important, Congruency also had an impact on RTs, $\mathrm{F}(1,13)=35.1, \mathrm{p}<.0001$. Responses were faster on congruent (365 ms) as compared to incongruent trials (394 ms). Importantly, priming effects depended on SOA, $\mathrm{F}(5,65)=130.3, \mathrm{p}<.0001$. Large positive priming effects occurred for the 0 -msSOA, $+108 \mathrm{~ms}$, whereas effects were much smaller but never inverse for the other SOAs $(+11,+11,+11,+15,+19 \mathrm{~ms}$, respectively). The numerical size of the priming effects suggests an increase with SOA, which also turned out to be statistically reliable as revealed by a separate analysis leaving out the 0 -ms-SOA condition, Congruency x SOA, F(4,52) $=3.0$, p 
$<$.05. Priming effects did not change with training as indexed by the interaction of Congruency and Session which failed to reach significance, $F(4,52)=2.2, p=.08$. This trend towards an interaction completely disappeared when restricting the analysis to the positive SOAs where we expected inverse priming to occur, $F(4,52)=1.6, p=.19$, suggesting that it seems to result in large part from a modulation of 0 -ms-SOA priming effects with Session. In accord, variance of priming effects across sessions was largest in the 0-ms-SOA condition (43 $\mathrm{ms}^{2}$ compared to $8,21,27,14,21 \mathrm{~ms}^{2}$ ). To complete the picture, the three-way interaction of Congruency, Session and SOA was far from being significant, $\mathrm{F}(20,260)<1$.

Accuracy data. Overall error rate was $3.2 \%$. SOA affected error rates, $\mathrm{F}(5,65)=7.6$, p < .0001. Subjects committed 4.5, 1.6, 2.5, 3.6, 3.4, $3.8 \%$ errors for the SOAs, respectively. This SOA effect was further modulated by Session, $\mathrm{F}(5,65)=2.1, \mathrm{p}<.01$. More important, error rates were affected by Congruency, $\mathrm{F}(1,13)=8.9, \mathrm{p}<.05$. As expected, these priming effects were influenced by SOA, $\mathrm{F}(20,260)=24.5, \mathrm{p}<.0001$. Large priming effects were observed for the 0 -ms-SOA, $+7.2 \%, \mathrm{t}(13)=7.0, \mathrm{p}<.0001$, and smaller effects for the other SOAs $(-$ $0.2,+0.4,-0.1,+0.2,+2.3 \%)$. At the $300-\mathrm{ms}-\mathrm{SOA}$ positive priming was significant, $\mathrm{t}(13)=$ $2.7, \mathrm{p}<.05$ but priming effects did not deviate from zero for all other SOAs, $|\mathrm{t}(13)|<1.7, \mathrm{p}>$ .13. The increase of priming effects with SOA was also reflected in the fact that the interaction of SOA and Congruency was still present when the analysis excluded the 0-msSOA condition, $\mathrm{F}(4,52)=5.2, \mathrm{p}<.01$. No further effects were significant. Especially, Session did not affect priming effects, $\mathrm{F}(4,52)<1$, even not in a three-way interaction with SOA, $\mathrm{F}(20,260)<1$.

Please insert Figure 13 about here

Prime recognition. Prime recognition performance depended on SOA when taking all SOA conditions into consideration, $\mathrm{F}(5,65)=31.4, \mathrm{p}<.0001$. Average performance was 94.2, 67.2, 69.7, 67.7, 71.5, 69.5\% for each SOA conditions, respectively, which exceeded chance level significantly for each SOA, $\mathrm{t}(13)>3.6, \mathrm{p}<.01$. When only positive SOAs were considered, which implied presentation of a masking stimulus, no SOA effect was found, $F(4,52)=2.1, p$ 
$=.09$. Individual recognition performance averaged across these masked prime conditions ranged from $49.6 \%$ to $98.1 \%$.

Prime recognition and priming effects. Effects of masked primes did not depend on Session but depended on SOA. Thus, we started by calculating correlations among priming effects averaged across sessions and prime recognition data for each SOA. For the RT data we found reliable positive correlations for each positive SOA, $\mathrm{r}>.58, \mathrm{p}<.05$. In the 0 -ms-SOA condition, this relation was absent, $\mathrm{r}=.04, \mathrm{p}=.89$. With respect to error rate data, no correlations were observed for positive SOAs, $|\mathrm{r}|<.22, \mathrm{p}>.45$. However, in the 0-ms-SOA condition, we obtained a negative correlation, $\mathrm{r}=-.73, \mathrm{p}<.01$. Next, we compared priming effects within positive SOA conditions among those subjects showing subliminal and supraliminal prime perception according to the two criteria described in Experiment 1. Individual performance averaged across positive SOAs (100-300 ms) was compared to the criteria to divide the sample of participants. Considering RT data, a dependency of priming effects on prime visibility group was not found for the division according to the strict criterion, group x Congruency: $\mathrm{F}(1,12)=1.6, \mathrm{p}=.24$, but for according to the more liberal criterion, $\mathrm{F}(1,12)=4.9, \mathrm{p}<.05$. While no priming effects were observed among those subjects showing subliminal prime perception, all $|\mathrm{t}(13)|<1.8, \mathrm{p}>.13$, the remaining participants who were able to discriminate the prime to some degree showed positive priming effects, all $\mathrm{t}(13)<2.5, \mathrm{p}<.05$ (except for the 200-ms-SOA: $\mathrm{t}(13)=-2.3, \mathrm{p}=.06$ ). With respect to error rate effects, no group differences were found neither for the stricter, $\mathrm{F}(1,12)<$ 1 , nor for the more liberal criterion, $\mathrm{F}(1,12)=1.5, \mathrm{p}=.25$.

\section{Discussion}

Evidence from Experiment 1-8 suggested that inverse priming requires response-compatible stimuli that are holistically processed. Experiment 9 was designed to rule out two different alternative explanations. First, we aimed to investigate whether inverse priming also arises temporally delayed with non-compatible stimuli. Therefore, we varied the mask-target SOA and especially included large SOA values of up to $300 \mathrm{~ms}$ when the inverse priming effect with other stimuli has already decayed (Mattler, 2005; Sumner, \& Brandwood, 2008). However, we failed to observe inverse priming for the entire range of SOAs from 100 to 300 ms. Instead priming effects were small but reliably biased towards positive priming. If SOA had any effect, positive effects increased with increasing SOA. Second, theoretically inverse 
priming could result with all kind of stimuli after extensive training, thereby establishing stimulus-response mappings. Such learning of SR-associations has been considered to be crucial for priming effects to occur (Boy, \& Sumner, 2010). However, we failed to observe any effect of practice on priming effects. Priming effects remained rather stable, and in particular, we did not observe any tendency for a reversal of priming effects during the last sessions which might have indicated that training was not sufficient. Stable inverse priming effects across several training sessions have also been reported by others (Schlaghecken, et al., 2008). Thus, it seems rather unlikely that we missed inverse priming effects in the experiments employing non-arrow stimuli because the SOA was too short or because SRlearning was incomplete. 


\section{General Discussion}

The current study was initially driven by the question of whether inverse priming effects originate from perceptual or post-perceptual processes. Eimer (1999) was the first to investigate this question by comparing inverse effects of primes identical to the target as opposed to perceptually dissimilar ones. His results and subsequent attempts by Klapp and Hinkley (2002; and also Schlaghecken, \& Eimer, 2000) demonstrated a complete transfer of priming effects to these dissimilar targets pointing to a motor origin of the effect. However, since all these studies used relevant masks the transfer of inverse priming effects could have been simply due to a perceptual interaction of primes and masks. To rule out this possibility, we replicated the experiment with irrelevant masks. At first glance, we found perceptual and post-perceptual processes to be involved in inverse priming with such irrelevant masks because effects only partially appeared with lateral dissimilar targets. Absolute response speed, however, differed for the two types of target stimuli. This difference might also alter inverse priming effects because the temporal interval between prime presentation and response delivery is considered critical by at least one theoretical account on inverse priming (Schlaghecken, \& Eimer, 2002). This idea is based on electrophysiological findings of inverse priming (Eimer, \& Schlaghecken, 1998, 2003; Praamstra, \& Seiss, 2005). Analyzing LRPs, Eimer and Schlaghecken (1998), for instance, observed a bi-phasic pattern of response activations in inverse priming. Initially, the prime drove response activation processes. This activation phase was reversed after mask presentation. Thus, quick responses might fall into the initial prime-governed period yielding more positive priming effects whereas only slower responses are affected by the mask induced reversal of response activation. As predicted, only slowest responses to lateral targets $\left(60^{\text {th }}\right.$ and $80^{\text {th }}$ percentile of RT distribution) showed inverse priming reaching a similar magnitude as inverse priming with central targets. Thus, when controlling for absolute response latencies, priming effects seem to fully transfer to lateral targets. The same finding has been reported before by Schlaghecken and Eimer (2000) when using relevant masks. The current results extend these findings to irrelevant masks and suggest that at least inverse priming with double arrow stimuli seems to be a phenomenon completely originating from post-perceptual levels of processing making a perceptual contribution to the effect unlikely. These results are in line with further empirical evidence supporting the notion of a post-perceptual locus of inverse priming (Boy, \& Sumner, 2010; Klapp, \& Haas, 2005; Klapp, \& Hinkley, 2002, Schlaghecken, Klapp, \& Maylor, 2009). Together, these studies provide evidence for the view that inverse priming truly reflects motor control processes of unconsciously triggered response tendencies as proposed by the broad 
class of motor accounts of inverse priming which assume a mask-triggered inhibition mechanism (Klapp, \& Hinkley, 2002; Lleras, \& Enns, 2006; Jaśkowski, \& PrzekorackaKrawczyk, 2005; Schlaghecken, \& Eimer, 2002).

On the other hand, the finding that inverse priming originates at post-perceptual levels of processing conflicts with other research suggesting a perceptual basis of the effect (Krüger, et al., in press; Mattler, 2006, 2007). However, in all these studies prime stimuli were masked by a metacontrast-mask which comprised both stimulus alternatives. In contrast to the above mentioned studies (e.g. Eimer, 1999), however, masks did not result from a direct superposition of both prime alternatives such that mask presentation does not simply add the opposite prime. Nevertheless, they can be interpreted as a relevant mask because inverse priming could result from a perceptual interaction of prime and target rendering the primeopposite elements of the mask more salient (Lleras, \& Enns, 2004) which facilitates the recognition of the target stimulus. In contrast, inverse priming with arrow-like stimuli might yield the same behavioral outcome but the way how this outcome is generated is qualitatively different. As will be discussed below with respect to the current experiments, inverse priming with non-arrow stimuli might only be possible with relevant masks but disappears for irrelevant masks (e.g. Jaśkowski, \& Ślósarek , 2007) and no contribution of motor processes that completely determine the effect with arrows (irrespective of the mask) might be expected for such non-arrow stimuli. Therefore, results from studies on inverse priming should be carefully interpreted. Their generalizability might be limited to the particular prime (arrow vs. non-arrow) and mask stimuli (relevant vs. irrelevant) used.

\section{Stimulus Dependency of Inverse Priming}

This study was initially stimulated by the question of the origin of inverse priming. However, we thereby discovered an astonishing dependency of inverse priming effects on the prime stimuli used irrespective of whether targets were identical or dissimilar. We failed to find inverse priming with a large number of different stimuli including symmetrical objects that do not possess any natural mapping with the responses (Experiment 2,3) and stimuli that possess elements which are compatible to the spatial alignment of responses (Experiment 5, 6, 7, 9). Evidence for inverse priming was only obtained with arrows (Experiment 1,8) and with bar pairs (Experiment 4b) confirming previous findings (Sumner, 2008; Boy, et al., 2008; Boy and Sumner, 2010). It is noticeable that these inverse priming effects with bar pairs were 
comparatively small and they seemed to require more practice than the effects with double arrows to arise because effects were only present during the second session. These differences together with the fact that the bar pair stimuli are physically most similar to the mask elements among all other stimuli tested suggested that the primes perceptually interact with the masks in this case leading to a saliency bias for those elements of the mask being most distinct from the prime which in turn leads to a mask-induced inverse priming effect. One has to note in opposition to this interpretation, however, that the inclusion of the targetorientations in the mask does not further enlarge inverse priming effects (Sumner, 2008). With the other stimuli tested here, such a perceptual interaction seems to be completely impossible. In other words, masks were truly irrelevant for these stimuli and then only arrow stimuli produced inverse priming. In the literature, instances of inverse priming with nonarrow stimuli are rather rare. Exceptions include rectangular double brackets (Jaśkowski, \& Ślósarek , 2007) and arrow-like patterns of horizontal lines intersected by a vertical line on either the left or right side (Verleger, et al., 2005). Apart from these stimuli that also possess some of the typical characteristics of arrows, inverse priming has been demonstrated with Landolt ring stimuli (at least when prime duration was increased to $50 \mathrm{~ms}$; Schlaghecken et al., 2007). These stimuli are not composed of intersecting straight lines and are not overlearned indicators of 'left' and 'right' suggesting that the directional associations are not the decisive property of arrow stimuli making them such suitable for inverse priming. Instead, the special status of arrows seems to be due to their high spatial compatibility to the response set. This idea receives further support by the fact that a reversal of SR-mappings with arrows does not reduce inverse priming effects (Jaśkowski \& Ślósarek, 2007; O’Connor, \& Neill, 2011). In accordance, symmetrical spatially non-compatible stimuli produced inverse priming only when relevant masks were employed allowing for a mask-induced inverse priming effect (Jaśkowski, \& Ślósarek , 2007; Krüger, et al., 2010; Kiesel, Berner, \& Kunde, 2008; Mattler, 2006; 2007). Supposedly, such a mask-induced effect also produced inverse priming in Experiment 4b. But why, then, did we fail to find any evidence for inverse priming with stimuli that also possess a high degree of spatial response compatibility? The decisive feature distinguishing tilted exclamation marks (Experiment 5), laterally displaced crosses (Experiment 6) or the complex barbell-like objects (Experiment 7) from arrows or Landolt rings might be that they are not treated as holistic entities by the visual system. Instead, they are more an assembly of single features. Instead of the identification of the target object, the task merely requires reporting the spatial position of the critical feature within this assembly. However, the idea that only holistic objects are required to produce inverse priming with 
irrelevant masks is preliminary and future experiments have to isolate the crucial characteristic of the stimuli suitable to generate the effect. If the interpretation is correct, spatially compatible stimuli that are holistically processed should lead to inverse priming. Stimuli like the lowercase letters ' $d$ ' and ' $b$ ' seem to be adequate for this purpose. Furthermore, faces are known to be processed as entities by the visual system (Robbins, \& McKone, 2003; Schiltz, \& Rossion, 2006). In studies on spatial attention faces with the eye's gaze directed either to the left or right have been successfully employed to automatically shift attention (e.g. Kuhn, \& Kingstone, 2009) even when these stimuli remain unconsciously (Sato, Okada, \& Toichi, 2007). Another promising way might be the manipulation of the instructions. Such top-down effects are known to alter visual perception (e.g. Liepelt, \& Brass, 2010). For instance, introducing the barbell-like objects as fish swimming left- or rightward might induce holistic visual processing which in turn allows for inverse priming.

Possible objections against the absence of inverse priming with non-arrow stimuli

The conclusion that inverse priming with irrelevant masks only works with spatial-compatible stimuli that are holistically processed might be inappropriate because it is of course impossible to proof the absence of inverse priming with other stimuli. One might argue that inverse priming exists with such stimuli but that we just failed to discover it because the effect obeys to different laws and the conditions triggering it were not met in the current study. Two such objections were considered in Experiment 9. First, inverse priming might necessitate more time to fully develop with other non-arrow stimuli. However, in Experiment 9 we did not observe inverse priming across the entire SOA range from 100 to $300 \mathrm{~ms}$ rendering this argument rather unlikely. Alternatively, one might argue that SR-mappings have to be established as prerequisite for inverse priming to occur (Boy, \& Sumner, 2010). Thus, practice should be critical for inverse priming. However, even after five sessions with 576 trials each performed on separate days no evidence for inverse priming was found.

Other potential confounding factors which potentially explain the absence of inverse priming with non-arrow stimuli include prime visibility or the overall response speed which turned out to be crucial for inverse priming with double arrows, too (see Experiment 1).

Prime visibility has previously been deemed crucial (Eimer, \& Schlaghecken, 2002) or at least considered as a moderating factor for inverse priming (Schlaghecken, et al., 2008; Sumner, 2007). Therefore it seems plausible that possible differences in prime recognition 
performance could account for the differences in priming effects. However, visibility cannot explain the absence of inverse priming with non-arrow stimuli, first, because the average visibility did not vary across the experiments (all experiments with lateral targets: $F(7,120)=$ $1.7, \mathrm{p}=.11$ ). In particular, visibility was not extremely low for arrow stimuli (double arrows: $59.6 \%$ and single: $59.8 \%$ ) compared to the other stimuli (average performance ranged from $52.4 \%$ in Experiment 2 to $68.0 \%$ in Experiment 7) which is expected to facilitate the occurrence of inverse priming (Sumner, 2007). Second, because prime visibility largely varied across subjects we analyzed priming effects separately for those subjects being unable to identify the primes in the prime recognition session. Even when a rather conservative criterion was adopted for subliminal prime perception (less than $53.5 \%$ of correctly identified primes) we failed to find inverse priming among these subjects. All these findings seem to rule out that different prime visibility was responsible for the absence of inverse priming effects.

Apart from prime visibility, overall RTs varied between the experiments. And in Experiment 1, we found that inverse priming effects depended on absolute response speed (cf. Schlaghecken \& Eimer, 2000). In line with previous LRP-findings (e.g. Eimer, \& Schlaghekcen, 1998) inverse priming effects occurred only for slowest responses. In the current experiments response speed to central targets changed markedly (only those experiments including lateral targets: $\mathrm{F}(7,120)=16.6, \mathrm{p}<.0001)$. Mean RTs ranged from 322 (Experiment 6) to $474 \mathrm{~ms}$ (Experiment 2). Response speed to double arrows (368 ms) and single arrows (391 ms) was somehow intermediate providing a first hint for the notion that we did not fail to find inverse priming with non-arrow stimuli simply because motor inhibition required more time to produce inverse prime with such stimuli. Because then, the occurrence of inverse priming for the arrow pattern stimuli from Experiment 2 should be especially likely. Apart from this global comparison between stimuli, we analyzed priming effects across different RT bins in the RT distributions which largely overlap between the experiments. Thus, if absolute response speed is critical, inverse priming effects should be found for at least some part of the RT distribution, especially that part overlapping with the RT distribution obtained in Experiment 1. We performed the same analysis which successfully indicated the dependency of priming effects with arrows in Experiment 1. To this end, we compared performance on congruent and incongruent trials for 19 percentiles of the RT distribution $\left(5^{\text {th }}\right.$, $10^{\text {th }}, \ldots, 95^{\text {th }}$ ) separately using t-tests for Experiment 2 to 9 . For most experiments, priming effects depended on response speed as indexed by an interaction of the Percentile factor and Congruency in a repeated-measures ANOVA (Experiment 3, 4, 5, 7 and 8: $\mathrm{F}(18,234)>1.9, \mathrm{p}$ 
$<.02$ ) but not for all of them (Experiment 2 and 6: $F(18,234)<0.6, p>.90$ ). Most importantly, however, we did not find inverse priming for any of the 19 percentiles for any stimulus in the first session (all $\mathrm{t}(13)>-1.4, \mathrm{p}>.18$ ) and almost also not in the second session (all $\mathrm{t}(13)>-2.2, \mathrm{p}>.05$ except for the $85^{\text {th }}$ percentile in Experiment $3: \mathrm{t}(13)=-2.4, \mathrm{p}=.034$, "neighboring percentiles" did not reach significance). Therefore, it seems rather unlikely that differences in response speed could account for the absence of inverse priming with nonarrow stimuli.

To sum up, we can be fairly confident that the observed stimulus dependency of inverse priming effects is simply a result of differences in prime visibility or response speed across arrow and non-arrow stimuli. Furthermore, extended practice or prolonged mask-target SOAs do not seem to increase the probability for inverse priming to occur with non-arrow stimuli further encouraging the conclusion that inverse priming effects with irrelevant masks are limited to spatially compatible object-like stimuli. Implications for a mechanism underlying inverse priming are discussed in the following section.

Why is inverse priming restricted to response compatible objects?

The idea that response compatible objects are processed on a distinct route is rather old (for a review see: Hommel, 2000). Dual route models accommodate this special status of response compatible stimuli by assuming an automatic, unconditional route for such stimuli in opposition to a controlled one relying on short-term binding of perceptual input and motor output which involves strategic control. Kornblum, Hasbroucq and Osman (1990) proposed the dimensional overlap model, a prominent representative of such dual route modals of SRtranslation. Whenever stimuli and response vary along a common underlying dimension - a situation called dimensional overlap - compatibility affects performance. Then, stimuli produce a rapid automatic response activation which is verified by a controlled response selection process operating in parallel. Both processes yield converging evidence when the compatible response is assigned to the current stimulus (e.g. left response to leftward pointing arrow) which leads to a quick response initiation. The involvement of automatic stimulus processing might constitute a prerequisite for inverse priming effects. Various studies demonstrated evidence for the view that in particular arrows are processed automatically. Eimer $(1993,1995)$ found early LRP-deflections consistent with the orientation of an arrow pre-cue even though it was completely useless in predicting the response to an upcoming 
target stimulus. These effects seemed to be independent of the instructions and subjective expectations. Strikingly, the arrow precues even activated the compatible response when the likely response to the target was contralateral (75\% invalid cues). In contrast, color precues even though predictive for the correct response ( $75 \%$ valid) failed to elicit such early response preparation processes.

Similar automatic effects of arrows have been repeatedly reported in studies on spatial attention. Pratt and Hommel (2003) demonstrated reflexive orientation of attention according to the orientation of an arrow (see also Pratt, Radulescu, Guo, \& Hommel, 2010). Seemingly the orientation of arrows cannot be ignored automatically inducing a shift of spatial attention even when they are counter-predictive for the likely position of a following target stimulus (Tipples, 2008). Before, such reflexive attentional shifts have been considered to be unique to biologically relevant cues like eye gaze (Friesen, Ristic, \& Kingstone, 2004) but numerous studies demonstrated comparable effects of arrow precues on overt and covert attention (Kuhn, \& Kingstone, 2009) even for arrows rendered invisible by backward masking (Cole, \& Kuhn, 2010). Premotor theories of attention directly relate attentional shifts to according motor planning processes (Rizzolatti, Riggio, Dascola, \& Umiltà, 1987). It might be that arrows automatically induce such a cascade of processes involving attentional shifts and motor planning which might constitute a prerequisite the inhibitory mechanism involved in inverse priming to start to operate.

\section{Conclusion}

Inverse priming seems to be a quite diverse phenomenon. When arrows (or probably also other response-compatible objects) are employed as primes and targets the effect seems to be completely due to motor control processes irrespective of the mask design. Relevant masks only additionally foster these motor processes leading to larger effects. In contrast, noncompatible stimuli seem to require relevant masks in order to produce inverse priming. Then, inverse priming originates at purely perceptual levels of processing. Thus, even though behavioral outcomes are essentially the same generalizability of results from experiments on inverse priming seem to be limited to the category of prime- and mask-stimuli used. 


\section{References}

Boy, F., Clarke, K., \& Sumner, P. (2008). Mask stimulus triggers inhibition in subliminal visuomotor priming. Experimental Brain research, 190, 111-116.

Boy, F., Evans, C.J., Edden, R.A.E., Singh, K.D., Husain, M., \& Sumner, P. (2010). Individual differences in subconscious motor control predicted by GABA concentration in SMA. Current Biology, 20, 1779-1785.

Boy, F. Husain, M., Singh, K.D., \& Sumner, P. (2010). Supplementary motor area activations in unconscious inhibition of voluntary action. Experimental Brain Research, 206, 441-448.

Boy, F., Husain, M., \& Sumner, P. (2010). Unconscious inhibition separates two forms of cognitive control. Proceedings of the Royal Society: Biological Sciences, 107 (24), 1113439.

Boy, F., \& Sumner, P. (2010). Tight coupling between positive and reversed priming in the masked priming paradigm. Journal of Experimental Psychology: Human Perception and Performance, 36(4), 892-905.

Cole, G.C., \& Kuhn, G. (2010). What the experimenter's prime tells the observer's brain. Attention, Perception, \& Psychophysics, 72 (5), 1367-76.

Eimer, M. (1993). Spatial cuing, sensory gating and selective response preparation: an ERPstudy on visuo-spatial orienting. Electroencephalography and clinical Neurophysiology, $88,408-420$.

Eimer, M. (1995). Stimulus-response compatibility and automatic response activation: evidence from psychophysiological studies. Journal of Experimental Psychology: Human Perception and Performance, 21(4), 837-854.

Eimer, M. (1999). Facilitatory and inhibitory effects of masked prime stimuli on motor activation and behavioural performance. Acta Psychologica, 101, 293-313.

Eimer, M. \& Schlaghecken, F. (1998). Effects of masked stimuli on motor activation: Behavioral and electrophysiological evidence. Journal of Experimental Psychology: Human Perception and Performance, 24, 1737-1747. 
Eimer, M., \& Schlaghecken, F. (2002). Links between conscious awareness and response inhibition: Evidence from masked priming. Psychonomic Bulletin \& Review, 9, 514-520.

Eimer, M., \& Schlaghecken, F. (2003). Response facilitation and inhibition in subliminal priming. Biological Psychology, 64, 7-26.

Eimer, M. \& Schubö, A., \& Schlaghecken, F. (2002). Locus of inhibition in the masked priming of response alternatives. Journal of Motor Behaviour,34, 3-10.

Eriksen, C., \& Hoffman, J.E. (1972). Some characteristics of selective attention in visual perception determined by vocal reaction time. Perception and Psychophysics, 11, 169171.

Friesen, C.K., Ristic, J., \& Kingstone, A. (2004). Attentional effects of counterpredictive gaze and arrow cues. Journal of Experimental Psychology: Human Perception and Performance, 30, 319-329.

Jaśkowski, P. (2007). The effect of nonmasking distractors on the priming of motor responses. Journal of Experimental Psychology: Human Perception and Performance, 33, 456-468.

Jaśkowski, P. (2008). The negative compatibility effect with nonmasking flankers: A case for mask-triggered inhibition hypothesis. Consciousness and Cognition, 17, 765-777.

Jaśkowski, P. (2009). Negative compatibility effect: the object-updating hypothesis revisited. Experimental Brain Research, 193, 157-160.

Jaśkowski, P., Biłuńska, A., Tomanek, M., \& Verleger, R. (2008). Mask- and distractortriggerd inhibitory processes in the priming of motor responses: An EEG study. Psychophysiology, 45, 70-85.

Jaśkowski, P., \& Przekoracka-Krawczyk, A. (2005). On the role of mask structure in subliminal priming. Acta Neurobiologiae Experimentalis, 65, 409-417.

Jaśkowski, P., \& Ślósarek, M. (2007). How important is the prime's gestalt for subliminal priming? Consciousness \& Cognition, 16, 485-497.

Hommel, B. (2000). The prepared reflex: Automaticity and control in stimulus-response translation. In S. Monsell \& J. Driver (eds.), Control of cognitive processes: Attention and performance XVIII (pp. 247-273). Cambridge, MA: MIT Press. 
Huber, D.E. (2008). Immediate priming and cognitive aftereffects. Journal of Experimental Psychology: General, 137, 324-347.

Kornblum, S., Hasbroucq, T., \& Osman, A. (1990). Dimensional overlap: cognitive basis for stimulus-response compatibility - A model and taxonomy. Psychological Review, 97(2), 253-270.

Kiesel, A., Berner, M.P., \& Kunde, W. (2008). Negative congruency effects: A test of the inhibition account. Consciousness and Cognition, 17, 1-21.

Klapp, S. T. (2005). Two versions of the negative compatibility effect: Comment on Lleras and Enns (2004). Journal of Experimental Psychology: General, 134, 431-435.

Klapp, S. T., \& Haas, B. W. (2005). Nonconscious influence of masked stimuli on response selection is limited to concrete stimulus-response associations. Journal of Experimental Psychology: Human Perception and Performance, 31, 93-209.

Klapp, S. T., \& Hinkley, L. B. (2002). The negative compatibility effect: Unconscious inhibition influences reaction time and response selection. Journal of Experimental Psychology: General, 131, 255-269.

Klauer, K.C., \& Dittrich, K. (2010). From sunshine to double arrows: An evaluation window account of negative compatibility effects. Journal of Experimental Psychology: General, $139,3,490-519$.

Kouider, S., \& Dehaene, S. (2007). Levels of processing during non-conscious perception: a critical review of visual masking. Philosophical Transactions of the Royal Society B: Biological Sciences, 362, 857-875.

Krüger, D., Klapötke, S., \& Mattler, U. (in press). PRP-paradigm provides evidence for a perceptual origin of the negative compatibility effect. Consciousness and Cogntion.

Kuhn, G., \& Kingstone, A. (2009). Look away! Eyes and arrows engage oculomotor responses automatically. Attention, Perception, \& Psychophysics, 71(2), 314-327.

Liepelt, R., \& Brass, M. (2010). Top-down modulation of motor priming by belief about animacy. Experimental Psychology, 57(3), 221-227.

Lingnau, A., \& Vorberg, D. (2005). The time course of response inhibition in masked priming. Perception and Psychophysics, 67(3), 545-557. 
Lleras, A. \& Enns, J.T. (2004). Negative compatibility or object updating? A cautionary tale of mask-dependent priming. Journal of Experimental Psychology: General, 133, 475-493.

Lleras, A. \& Enns, J.T. (2005). Updating a cautionary tale of masked priming: Reply to Klapp (2005). Journal of Experimental Psychology: General, 134, 436-440.

Lleras, A., \& Enns, J. T. (2006). How much like a target can a mask be? Geometric, spatial, and temporal similarity in priming: A reply to Schlaghecken and Eimer (2006). Journal of Experimental Psychology: General, 135, 495-500.

Mattler, U. (2003). Priming of mental operations by masked stimuli. Perception and Pschophysics, 65, 167-187.

Mattler, U. (2005). Inhibition and decay of motor and non-motor priming. Perception and Pschophysics, 67, 285-300.

Mattler, U. (2006). On the locus of priming and inverse priming effects. Perception and Psychophysics, 68, 975-991.

Mattler, U. (2007). Inverse target- and cue-priming effects of masked stimuli. Journal of Experimental Psychology: Human Perception and Performance, 33, 83-102.

Nachev, P., Kennard, C., \& Husain, M. (2008). Functional role of the supplementary and presupplementary motor areas. Nature Reviews Neuroscience, 9, 856-869.

Praamstra, P., \& Seiss, E. (2005). The neurophysiology of response competition: motor cortex activation and inhibition following subliminal response priming. Journal of Cognitive Neuroscience, 17 (3), 483-493.

Pratt, J, \& Hommel, B. (2003). Symbolic control of visual attention: The role of memory and attentional control settings. Journal of Experimental Psychology: Human Perception and Performance,29, 835-845.

Pratt, J., Radulescu, P., Guo, R.M., \& Hommel, B. (2010). Visuospatial attention is guided by both the symbolic and the spatial proximity of selected arrows. Journal of Experimental Psychology: Human Perception and Performance, 36 (5), 1321-1324.

Przekoracka-Krawczyk, A., \& Jaśkowski, P. (2007). Perceptual learning can reverse subliminal priming effects. Perception \& Psychophysics, 69 (4), 558-566. 
O’Connor, P., \& Neill, W.T. (2011). Does subliminal priming of free response choices depend on task set or automatic response activation. Consciousness and Cognition, 20, 280-287.

Rizzolatti, G., Riggio, L, Dascola, I., \& Umiltà, C. (1987). Reorienting attention across the horizontal and vertical meridians: Evidence in favour of a premotor theory of attention. Neurophysiologica, 25, 31-40.

Robbins, R., \& McKone, E. (2003). Can holistic processing be learned for inverted faces? Cognition, 88, 79-107.

Sato, W., Okada, T., \& Toichi, M. (2007). Attentional shift by gaze is triggered without awareness. Experimental Brain Research, 183, 87-94.

Schiltz, C., \& Rossion, B. (2006). Faces are represented holistically in the human occipitotemporal cortex. Neuroimage, 32, 1385-1394.

Schlaghecken, F., \& Eimer, M. (2000). A central-peripheral asymmetry in masked priming. Perception \& Psychophysics, 62, 1367-1382.

Schlaghecken, F. \& Eimer, M. (2002). Motor activation with and without inhibition: evidence for a treshold mechanism in motor control. Perception and Psychophysics, 64, 148-162.

Schlaghecken F., Blagrove E., \& Maylor, E.A. (2007). Incidental learning of S-R contingencies in the masked prime task. Journal of Experimental Psychology: Human Perception and Performance, 33 (5), 1177-1188.

Schlaghecken F., Blagrove E., \& Maylor, E.A. (2008). No difference between conscious and nonconscious visuomotor control: evidence from perceptual learning in the masked prime task. Consciousness and Cognition. 17(1), 84-93.

Schlaghecken, F., Klapp, S.T., \& Maylor, E.A. (2009). Either or neither, but not both: locating the effects of masked primes. Proceedings of the Royal Society: Biological Sciences, 276, 515-521.

Schlaghecken, F., Rowley, L., Sembi, S., Simmons, R., \& Whitcomb, D. (2007). The negative compatibility effect: A case for self-inhibition. Advances in Cognitive Psychology, 3, 227-240. 
Schlaghecken, F., \& Eimer, M. (2006). Active masks and active inhibition: A comment on Lleras and Enns (2004) and on Verleger, Jaśkowski, Aydemir, van der Lubbe, and Groen (2004). Journal of Experimental Psychology. General, 135, 484-494.

Schlaghecken, F., Münchau, A., Bloem, B., Rothwell, J.C., \& Eimer, M. (2003). Slow frequency repetitive transcranial magnetic stimulation affects reaction times, but not priming effects, in a masked prime task. Clinical Neurophysiology, 14, 1272-77.

Sohrabi, A. \& West, R.L. (2009). Positive and negative congruency effects in masked priming: A neuro-computational model based on representation, attention and conflict. Brain Research, 1289, 124-132.

Sumner, P. (2007). Negative and positive masked priming - implications for motor inhibition. Advances in Cognitive Psychology, 3, 317-326.

Sumner, P. (2008). Mask-induced priming and the negative compatibility effect. Experimental Psychology, 55(2), 133-141.

Sumner, P., \& Brandwood, T. (2008). Oscillations in motor priming: positive rebound follows the inhibitory phase in the masked prime paradigm. Journal of Motor Behaviour, 40(6), 484-489.

Sumner, P., Nachev, P., Morris, P., Peters, A.M., Jackson, S.R., Kennard, C., \& Husain, M. (2007). Human medial frontal cortex mediates unconscious inhibition of voluntary action. Neuron, 54(5), 697-711.

Sumner, P., Tsai, P.-C., Yu, K., \& Nachev, P. (2006). Attentional modulation of sensorimotor processes in the absence of perceptual awareness. Proceedings of the National Academy of Sciences USA, 103 (27), 10520-25.

Tipples, J. (2008). Orienting to counterpredictive gaze and arrow cues. Perception and Psychophysics, 70 (1), 77-87.

Todd, J.T., \& van Gelder, P. (1979). Implications of a transient-sustained dichotomy for the measurement of human performance. Journal of Experimental Psychology: Human Perception and Performance, 5, 625-638.

Verleger, R., Görgen, S., \& Jaśkowski, P. (2005). An ERP indicator of processing of relevant gestalts in masked priming. Psychophysiology, 42, 677-690. 
Verleger, R., Jaśkowski, P., Aydemir, A., van der Lubbe, R.H.J. \& Groen, M. (2004).Qualitative differences between conscious and non-conscious processing? On inverse priming induced by masked arrows. Journal of Experimental Psychology: General, 133, 494-515. 
Figure Legends

Figure 1. Stimulus material used Experiment 1-9. Prime stimuli also appeared as central target stimuli on half of the trials. Otherwise a lateral target was presented (which is not depicted). The same irrelevant masks were used in each experiment except for Experiment 4.

Figure 2. Results of Experiment 1: Mean response times (lines) and error rates (bars) for central (solid line, black bars) and lateral targets (dashed line, grey bars). Inverse priming effects were more pronounced for central targets but partially transferred to lateral targets.

Figure 3. Scatterplots displaying individual inverse priming effects for central and lateral targets on RT data (left) and accuracy data (right). Regression functions are depicted as solid black lines. If priming effects fully transfer to lateral targets regression functions would be expected to have the same slope as the blue dashed line (slope of 1). However, the slope of the regression function was smaller in the RT domain (.83) and accuracy data domain (.44) indicating the partial transfer of priming effects to lateral targets.

Figure 4. Relation of priming effects and prime visibility. A) Scatterplots for prime recognition performance and priming effects on RTs (left) and error rates (right), respectively. Black dashed lines denote the two thresholds for subliminal prime perception. B) Mean priming effects on RTs (left) and error rates (right) within the good (green) versus poor (black) prime recognition performance group according to the two alternative divisions derived from two different criteria for subliminal prime perception. An intermediate group (red) was either assigned to the good performance group according to the stricter theoretical criterion $(53.5 \%)$ or to the poor performance group according to the more liberal empirical criterion $(63.9 \%)$. However, priming effects differed in a similar way between the two groups irrespective of the criterion. See subsection 'Prime recognition and priming effects' for further details.

Figure 5. Results of Experiment 2: RTs and error rates for central (solid lines and black bars) and lateral targets (dashed lines and grey bars) for the first (left panel) and second session (right panel). Primes did neither affect performance to central nor to lateral targets irrespective of the session.

Figure 6. Results of Experiment 3: RTs and error rates for central (solid lines and black bars) and lateral targets (dashed lines and grey bars) for the first (left panel) and second session (right panel). As in Experiment 2, prime-target congruency did not affect performance. 
Figure 7. Results of Experiment 4a: RTs and error rates for central (solid lines and black bars) and lateral targets (dashed lines and grey bars) for the first (left panel) and second session (right panel). Again, prime-target congruency did not affect performance.

Figure 8. Results of Experiment 5: RTs and error rates for central (solid lines and black bars) and lateral targets (dashed lines and grey bars) for the first (left panel) and second session (right panel). No evidence for inverse priming was found.

Figure 9. Results of Experiment 6: RTs and error rates for central (solid lines and black bars) and lateral targets (dashed lines and grey bars) for the first (left panel) and second session (right panel). Priming effects were positive but again we failed to find any evidence for inverse priming.

Figure 10. Results of Experiment 7: RTs and error rates for central (solid lines and black bars) and lateral targets (dashed lines and grey bars) for the first (left panel) and second session (right panel). If anything, priming effects were positive but again inverse priming was completely absent.

Figure 11. Results of Experiment 8: RTs and error rates for central (solid lines and black bars) and lateral targets (dashed lines and grey bars) for the first (left panel) and second session (right panel). Priming effects averaged across all participants were indifferent form zero. However, among those subjects being unable to discriminate the primes in the prime recognition session, evidence for inverse priming was indeed found.

Figure 12. Mean priming effects on RTs (left column) and error rates (right column) for the first (top panels) and second session (bottom panels) among the good (green) versus poor (black) prime recognition performance group. The intermediate group (red) is assigned to the group with supraliminal prime perception according to the stricter criterion $(53.5 \%)$ and to the subliminal prime perception group according to the more liberal criterion (63.9\%). Priming effects differed among the groups. Inverse priming was found on RTs and error rates (restricted to the second session) when adopting the stricter theoretical criterion.

Figure 13. Results of Experiment 9: RTs and error rates for central (solid lines and black bars) and lateral targets (dashed lines and grey bars) for session 1 to 5. Again, we failed to find any evidence for inverse priming. 
Figure 1

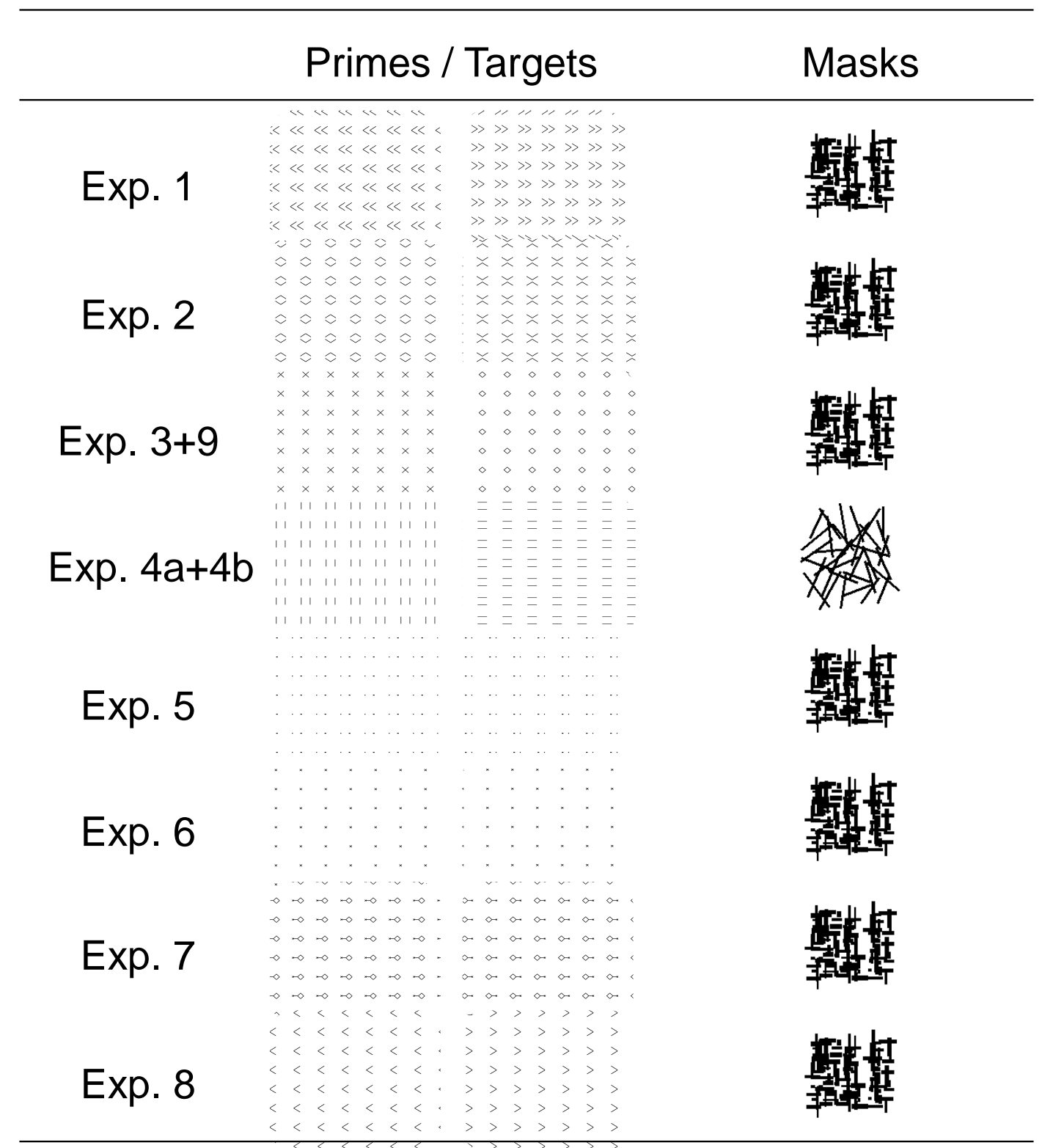


Figure 2

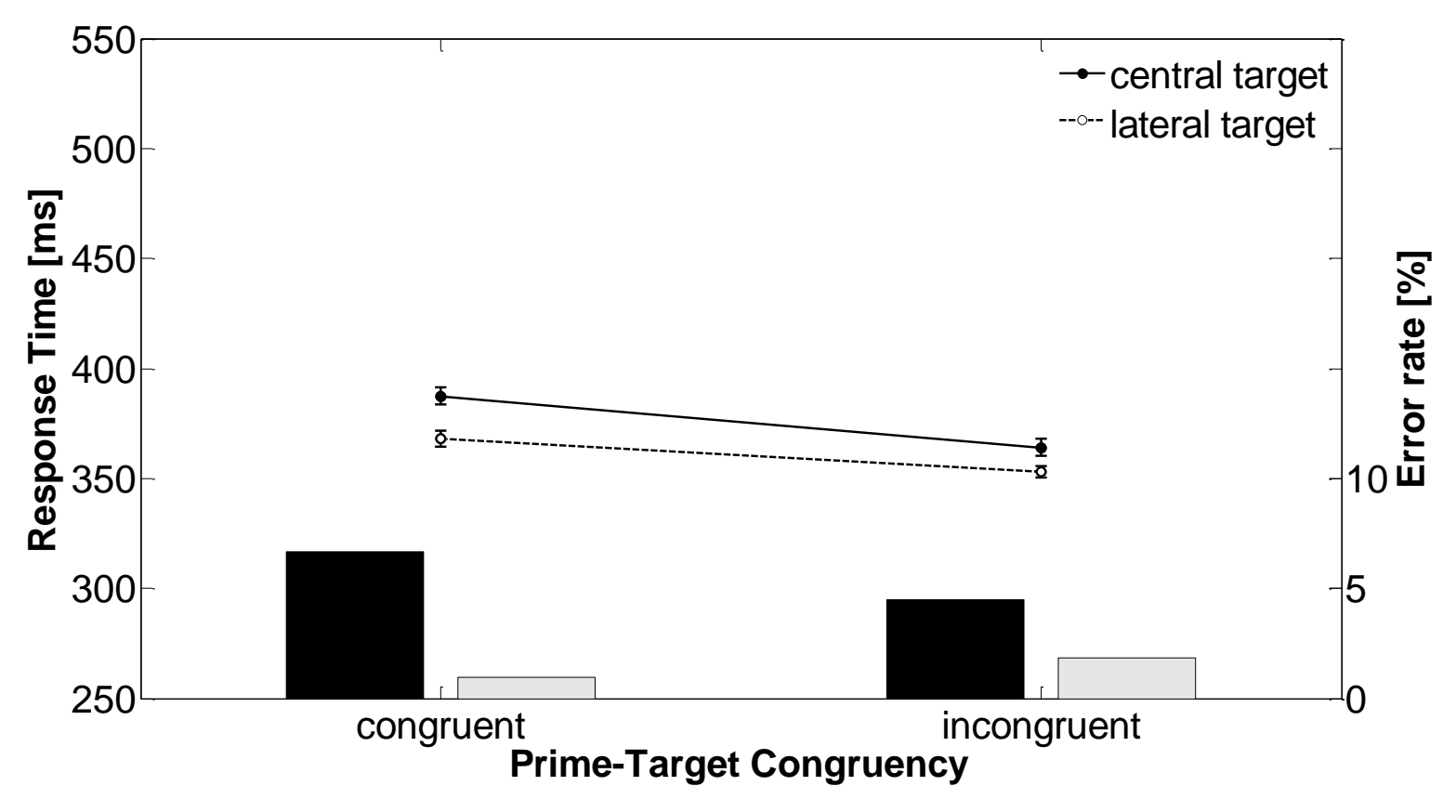


Figure 3

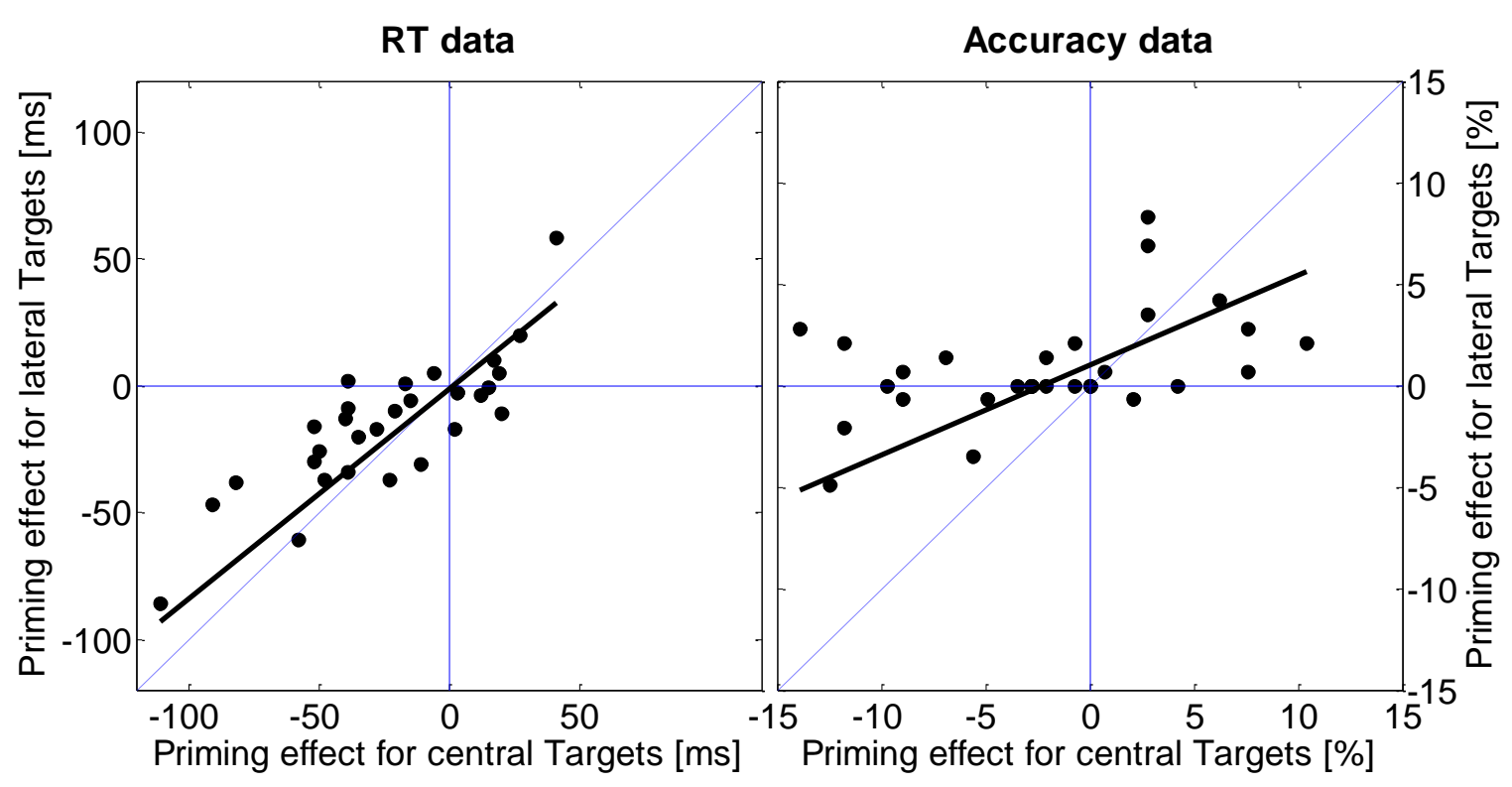


Figure 4

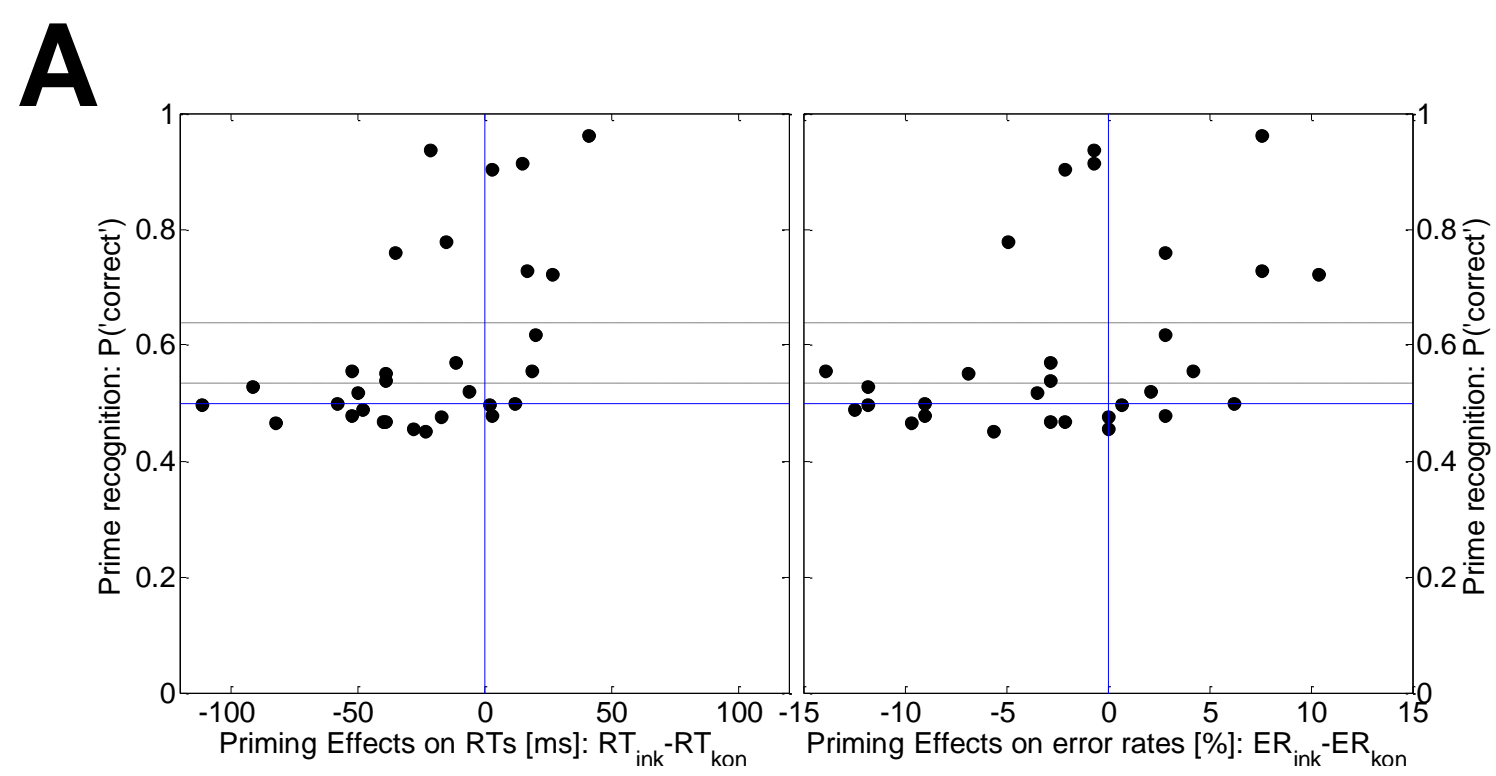

B

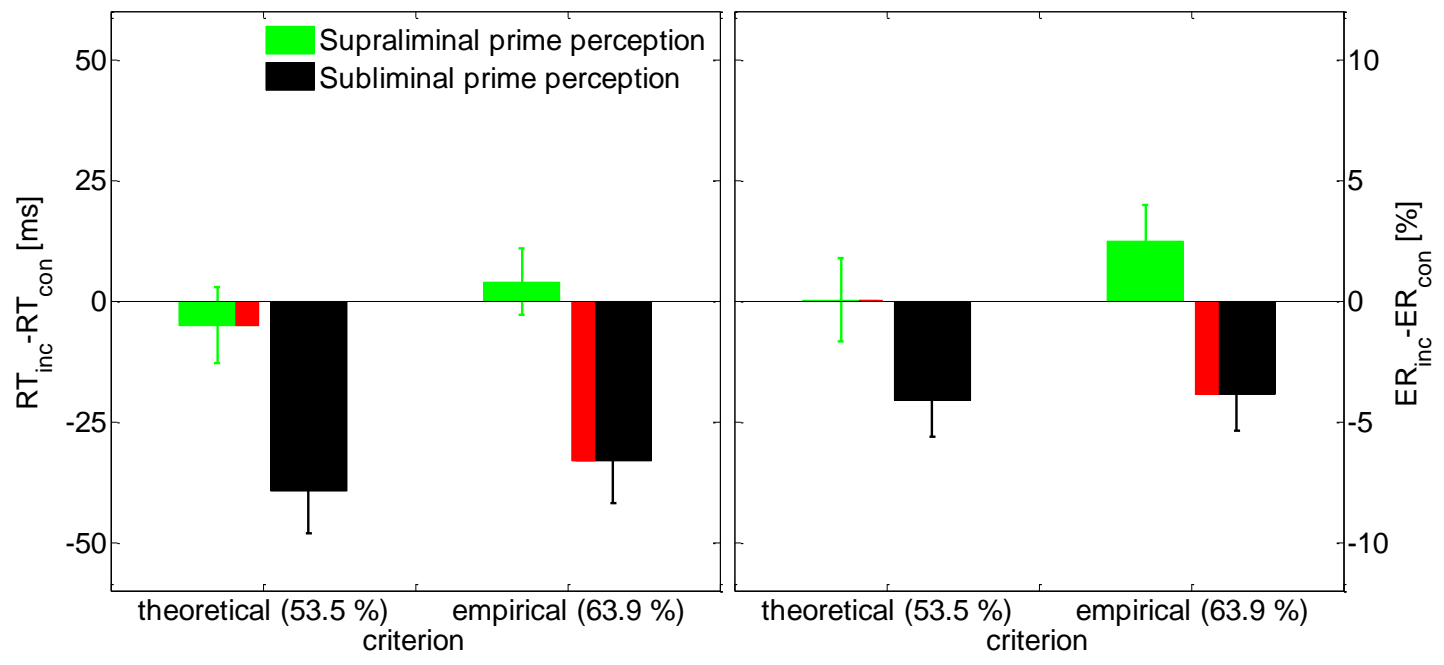


Figure 5

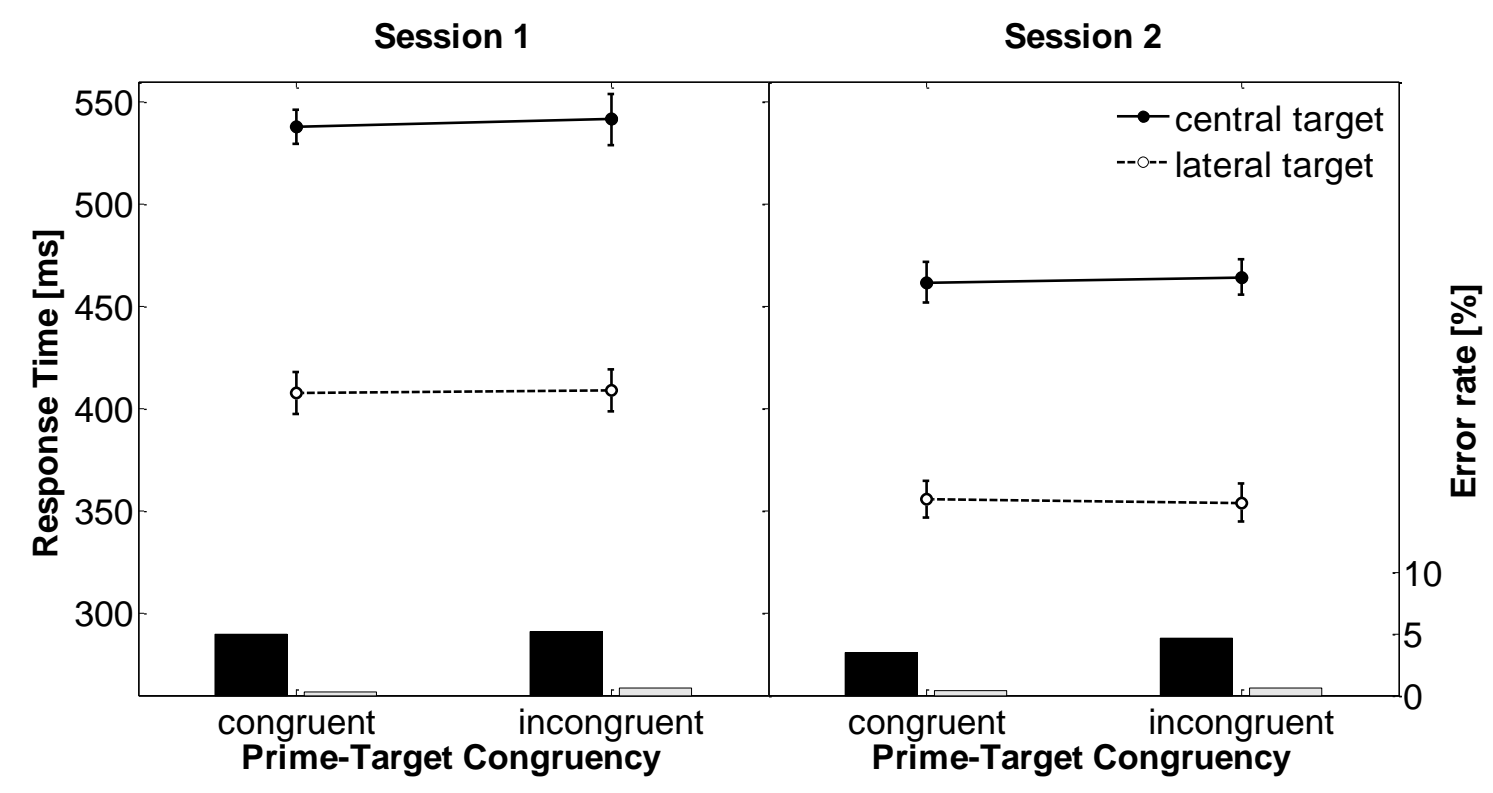


Figure 6

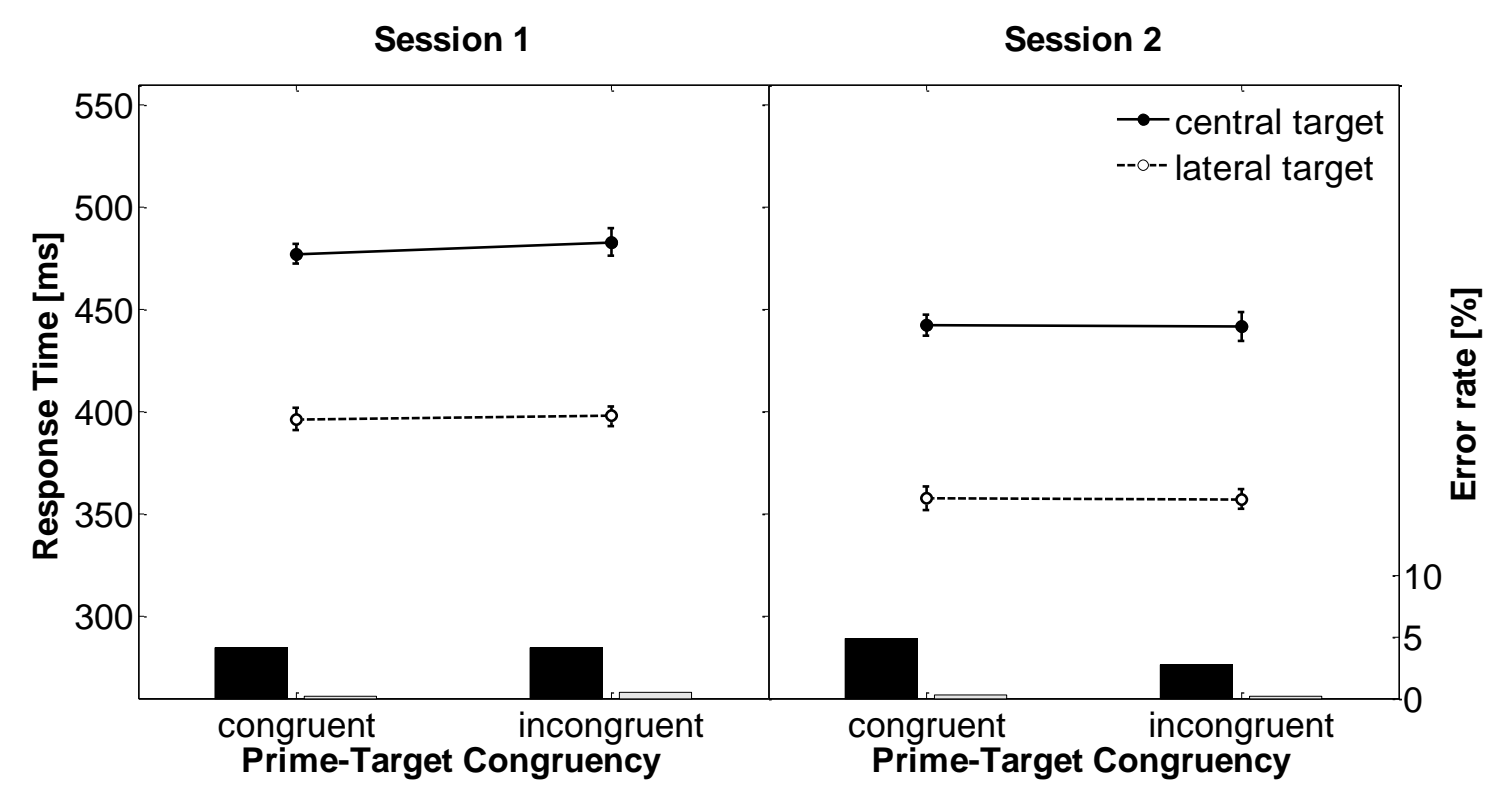


Figure 7

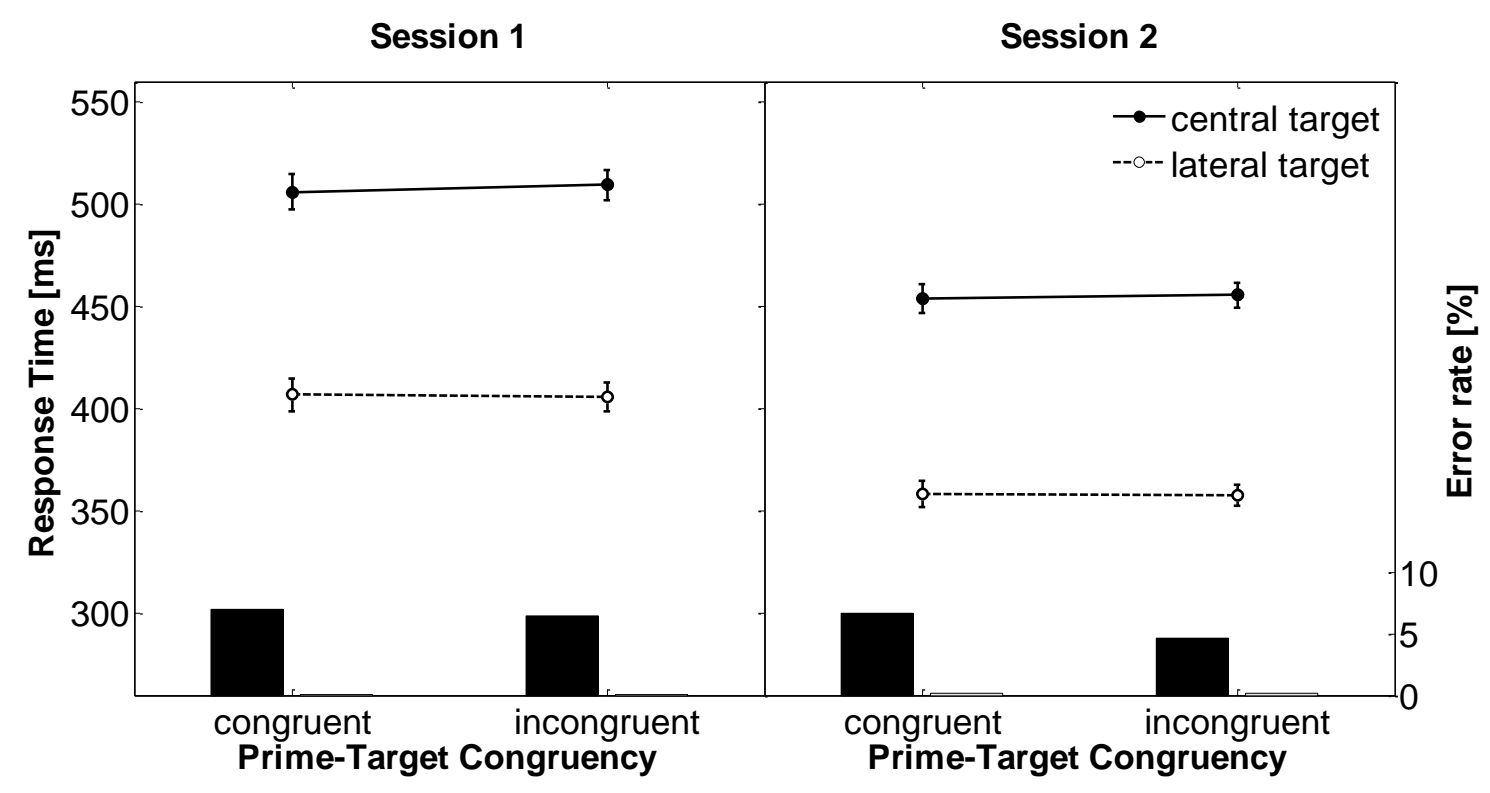


Figure 8

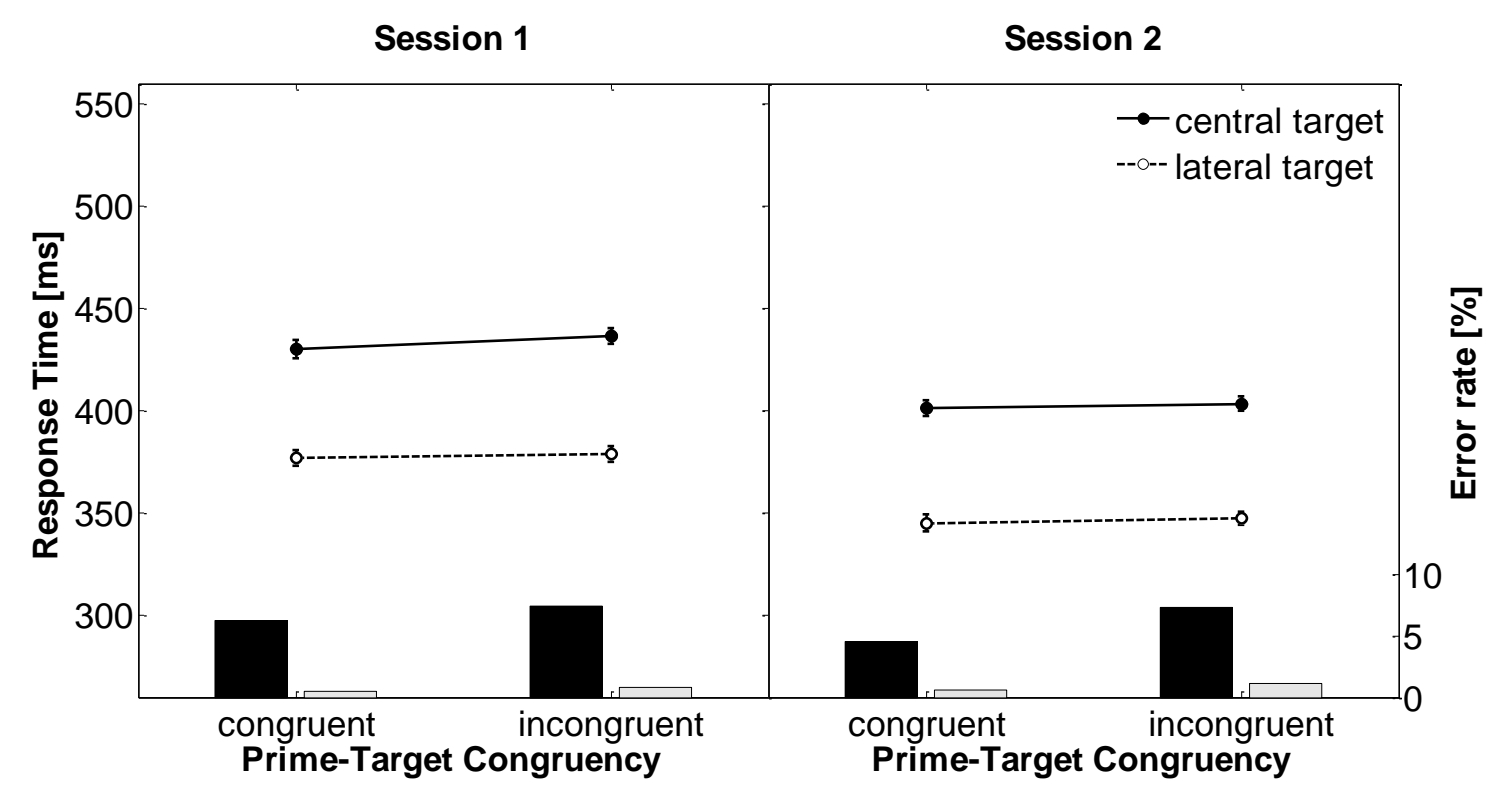


Figure 9

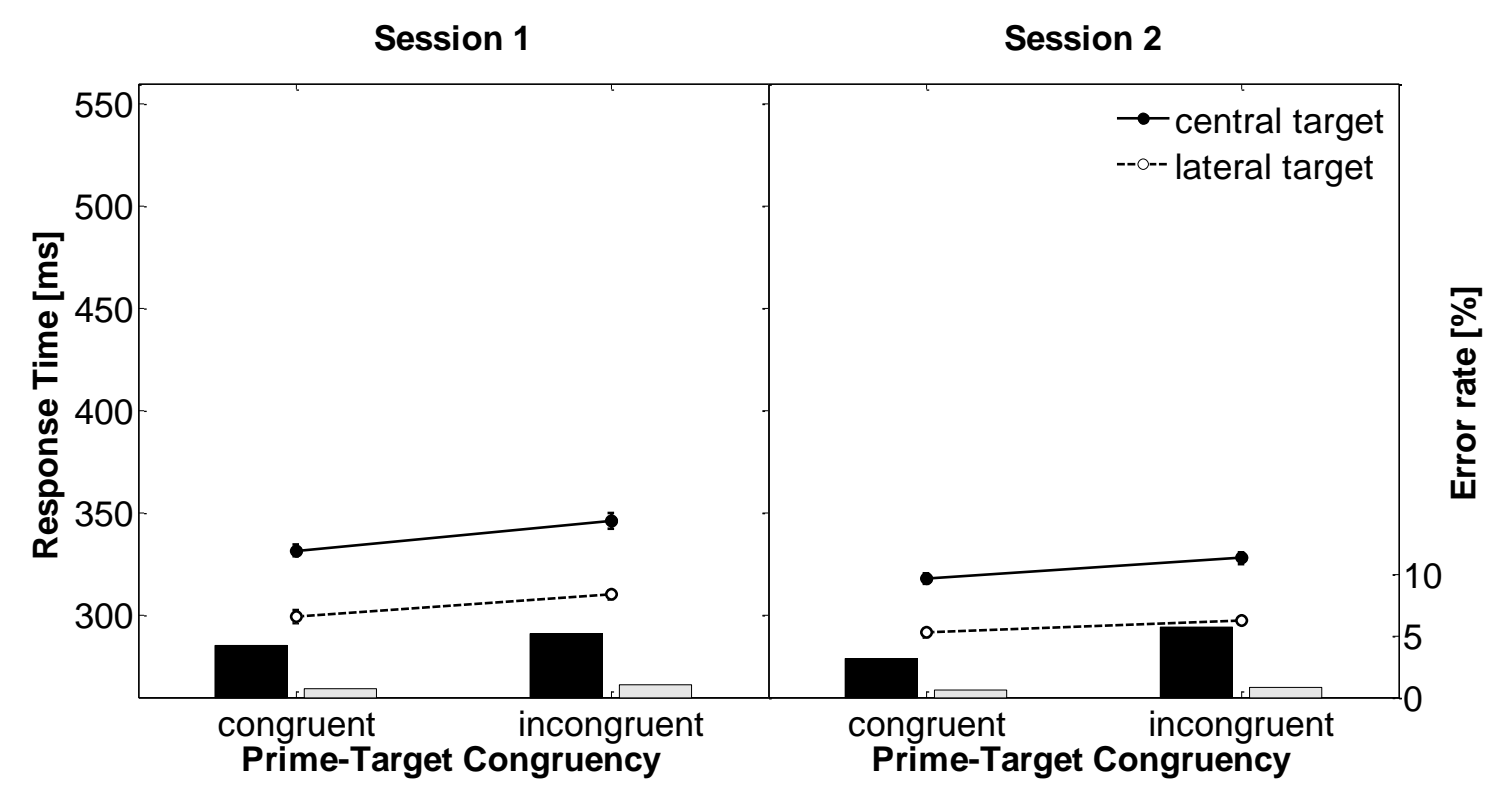


Figure 10

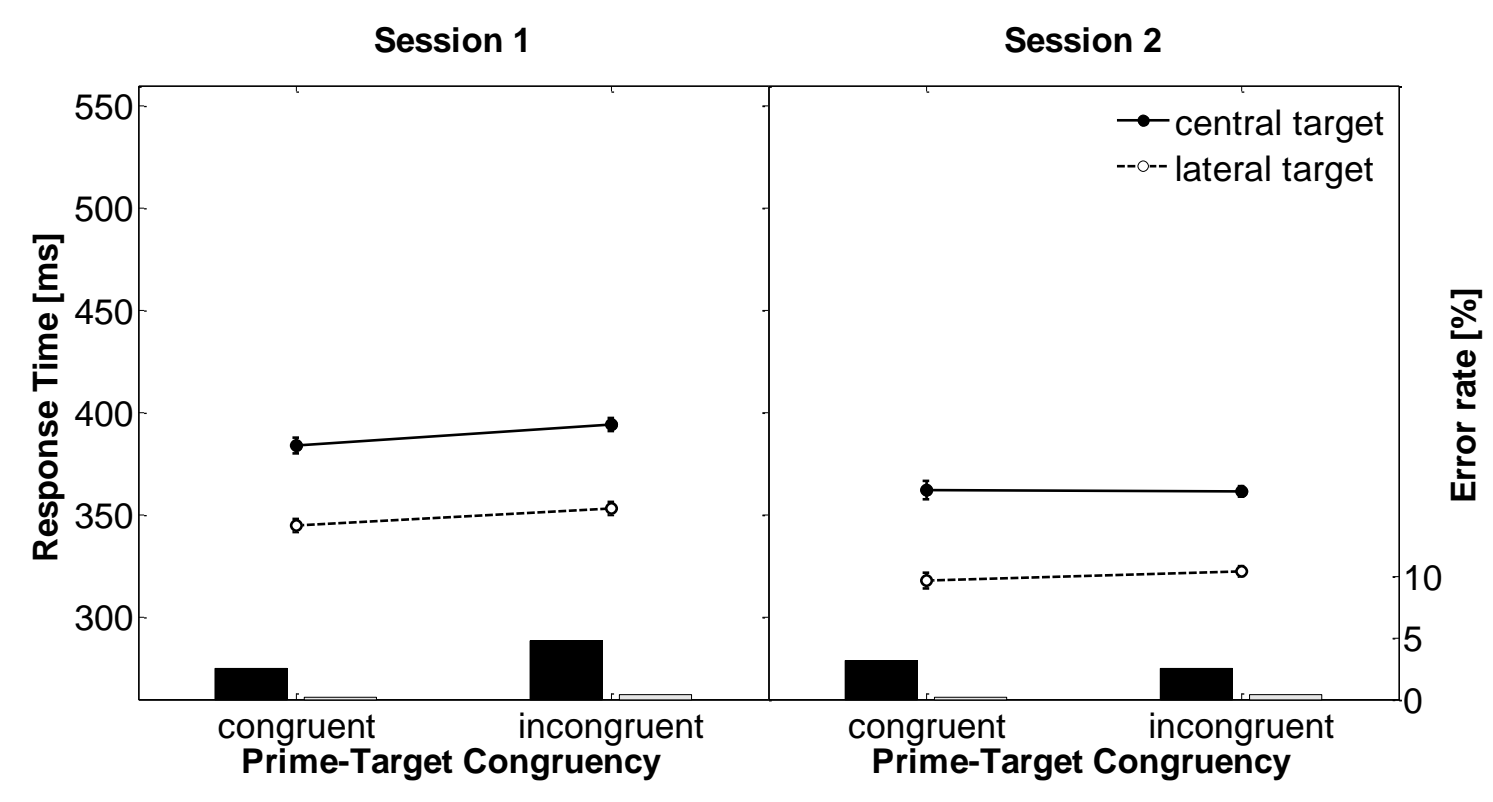


Figure 11

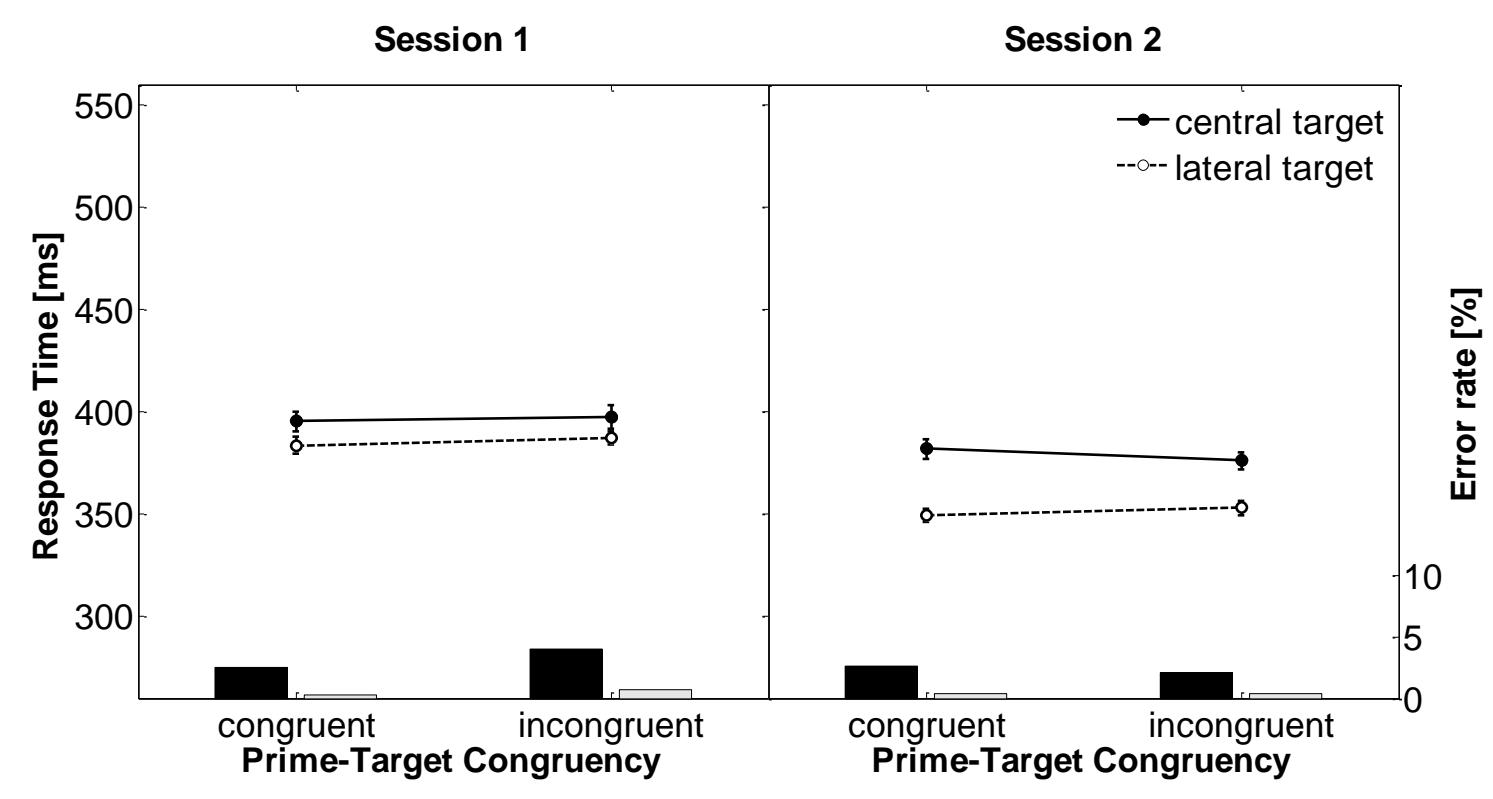


Figure 12

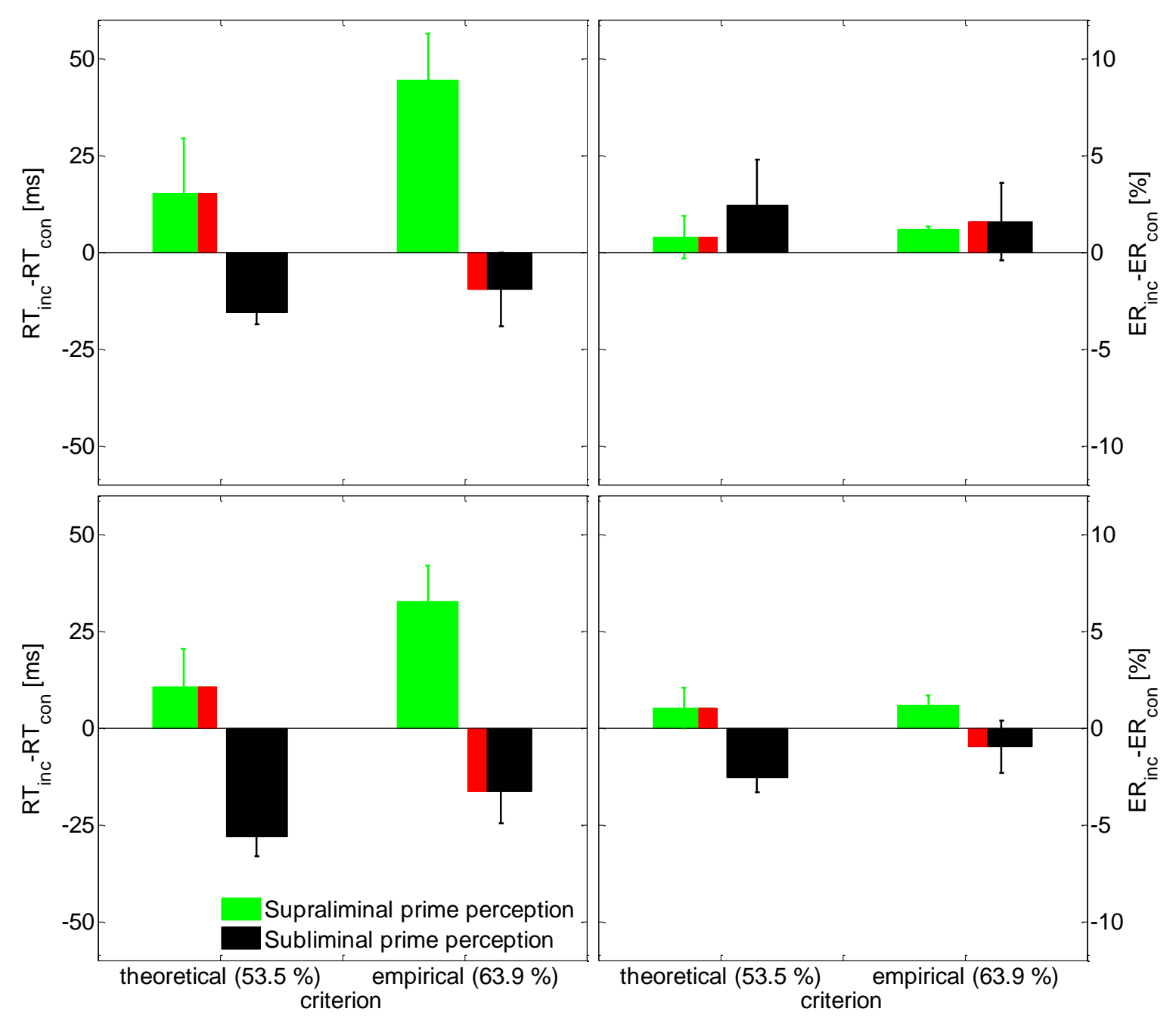


Figure 13

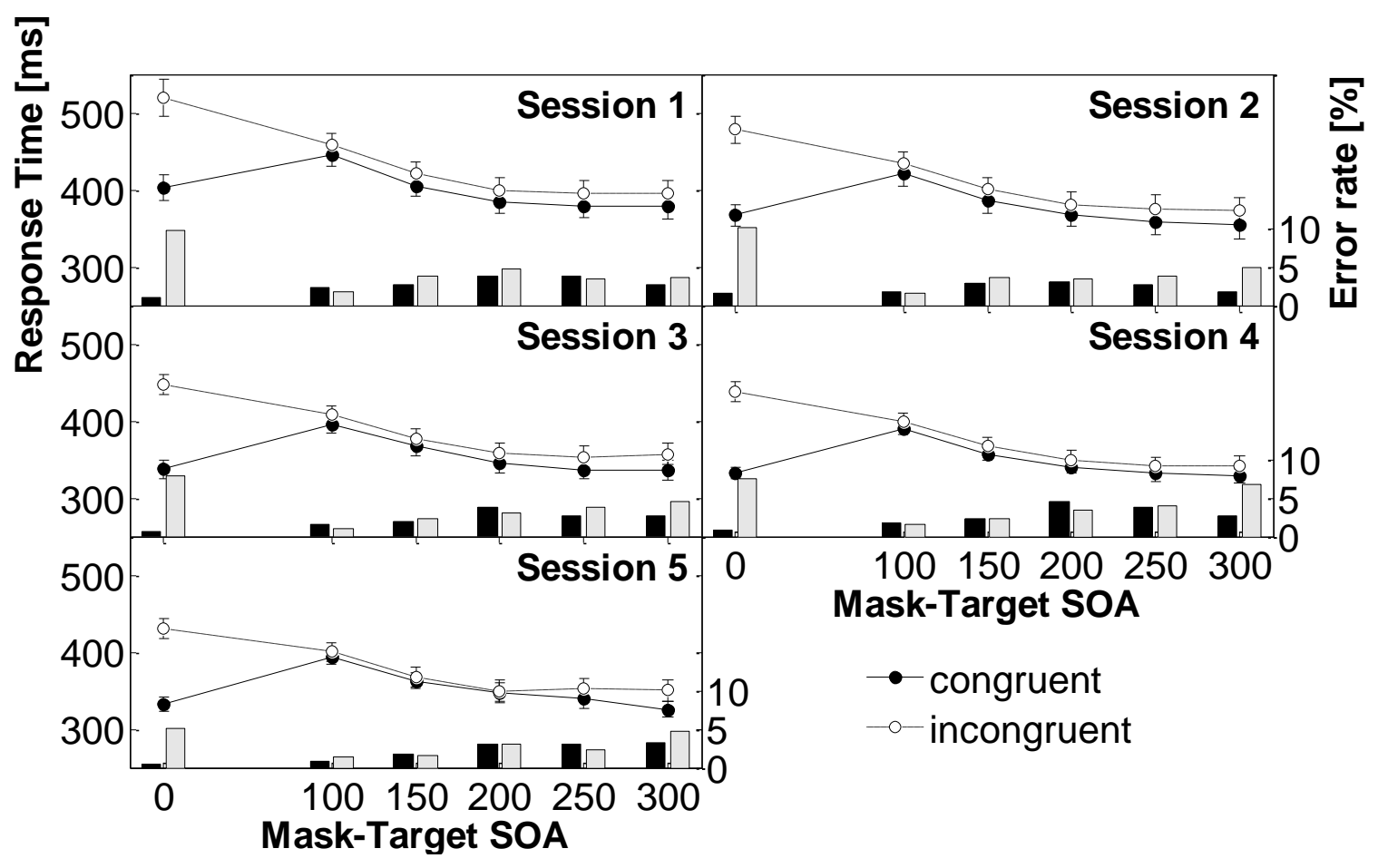




\section{Appendix IV}

Krüger, D., Mattler, U. (in prep). Inverse non-motor priming: Implications for the mechanisms of inverse priming. 


\title{
Inverse non-motor priming: implications for the mechanisms of inverse priming
}

\author{
Daniel Krüger \& Uwe Mattler
}

Georg-Elias-Müller Institute for Psychology, Georg-August University Göttingen

Send correspondence to:

Uwe Mattler

Georg-Elias-Müller Institute for Psychology

Georg-August University Göttingen

Gosslerstr. 14

D-37073 Göttingen

Germany

Email: uwe.mattler@psych.uni-goettingen.de

Keywords: priming, masking, consciousness, inhibition

Manuscript includes 4 Figures 


\begin{abstract}
Apart from positive priming effects, masked prime stimuli can impair responses to a subsequent target stimulus which shares response-critical features in contrast to a target assigned to the opposite response. This counterintuitive phenomenon is called inverse priming. We used a cue-priming paradigm to study the underlying mechanism of inverse priming for relevant and irrelevant masks. We found inverse cue-priming effects with both types of masks. When irrelevant masks were employed inverse priming was restricted to those subjects being unable to perceive the prime. The existence of inverse non-motor priming under conditions where simple perceptual interactions between the stimuli are ruled out as the source of inverse priming is at odds with the current view that inverse priming reflects motor inhibition. Alternatives are discussed.
\end{abstract}




\section{Introduction}

Unconscious information can influence overt behaviour. As demonstrated in numerous studies, a masked prime which can either be assigned to the same (congruent case) or the opposite response (incongruent case) compared to a subsequent target stimulus influences performance (e.g. Vorberg, Mattler, Schmidt, Heinecke \& Schwarzbach, 2003). In the traditional masked priming paradigm, the target also acts as backward-mask for the prime diminishing its visibility. Typically, performance benefits are observed for primes and targets which share task-relevant features, and performance costs if primes and target stimuli are incongruent. Strikingly, this pattern is reversed when the prime is followed by a neutral mask which in turn is followed by the target stimulus with a sufficiently long delay (about $100 \mathrm{~ms}$ ). This phenomenon has been termed inverse priming or the negative compatibility effect (Eimer \& Schlaghecken, 1998).

Originally inverse priming was ascribed to inhibitory processes that originate in the motor system (Klapp \& Hinkley, 2002; Schlaghecken \& Eimer, 2002; Schlaghecken, Bowman \& Eimer, 2006). Schlaghecken and Eimer (2002) proposed that the removal of prime-induced activity by the mask leads to self-inhibition of the primed response. Note that this assumption entails a causal link between prime visibility and the processes that lead to inverse priming: only effective masking causes the self-inhibition process.

The motor self-inhibition account has often been criticized for this assumption (e.g. Jaśkowski, \& Verleger, 2007). Even though there seems to be a positive correlation of priming effects (expressed as the difference between performance in the incongruent minus the congruent condition) with prime visibility (Lleras, \& Enns, 2006; Sumner, 2007) positive priming also occurred for invisible primes and, even more surprising, inverse priming was observed with completely unmasked primes (Jaśkowski, 2007; 2008; Jaśkowski, Biłuńska, Tomanek, \& Verleger, 2008; Lleras, \& Enns, 2006). This evidence challenged the view that the complete removal of the prime representation is a necessary prerequisite for inverse priming. Nevertheless, further accounts still postulated that the masking stimulus is of crucial importance for the reversal of priming effects even though these accounts did not focus on its masking property (Jaśkowski, \& Przekoracka-Krwczyk, 2005; Mattler, 2005; Lleras \& Enns, 2006). Instead every stimulus that is immediately following the prime and appears within the focus of attention might trigger the processes that lead to inverse priming (Jaśkowski, \& Verleger, 2007). Sumner (2007) summarized these accounts by their common assumption of stimulus-triggered instead of self-triggered inhibition. 
A completely different conception of inverse priming was introduced by Lleras and Enns (2004). They outlined one important commonality of the early studies on inverse priming: in the vast majority of experiments, masks were composed of the two stimulus alternatives employed as primes and targets (typically left- and right-pointing double arrows were superimposed, e.g. Eimer, \& Schlaghecken, 1998). These masks were built of task-relevant elements and therefore called relevant masks. Using such masks Lleras and Enns (2004) observed inverse priming while irrelevant masks, which exclusively consist of task-irrelevant elements, led to positive priming. Therefore, Lleras and Enns (2004) interpreted inverse priming within the framework of their Object Updating theory. Basically, they assume a saliency modulation of the mask elements caused by the prime that facilitates processing of an prime-incongruent target and disturbs processing of a congruent target. They postulate that the three stimuli presented in sequential order are integrated into a common internal representation. An iterative updating process of this representation yields a special accentuation of new elements in the changing visual input. If the mask is composed of both prime alternatives, the prime-opposite elements in the mask gain a relative saliency advantage which in turn produces the observable performance benefits for the processing of primeincongruent target stimuli. Thus, Lleras and Enns interpreted inverse priming as a positive priming effect generated by a subset of mask elements whose saliency is reinforced by a perceptual interaction of prime and mask.

For obvious reasons, Object Updating can only explain inverse priming with relevant masks because updating of new elements within a mask only built by task-irrelevant elements would not generate a bias for any response alternative. However, further research demonstrated that inverse priming also exists when irrelevant masks were applied (Eimer \& Schlaghecken, 2002; Klapp, 2005; Klapp \& Haas, 2005; Lleras \& Enns, 2005; Schlaghecken, \& Eimer, 2006; Schlaghecken et al., 2007; Sumner, 2008). This observation led to the assumption that more than a single mechanism produces inverse priming. Some researchers proposed that Object Updating is responsible for the generation of inverse priming when relevant masks are used and a separate inhibitory mechanism comes into play when irrelevant masks are used (Klapp, 2005). Instead of assuming two distinct mechanisms for each type of mask, one mechanism could contribute in both cases ("basic mechanism") while for relevant masks an "additional mechanism" like Object Updating is involved. This latter view is supported by the fact that inverse priming is usually larger when relevant masks are applied as compared to irrelevant masks (Eimer, \& Schlaghecken, 2006; Jaśkowski, 2007, 2008; Jaśkowski, \& Przekoracka-Krawczyk, 2005; Jaśkowski, Biłuńska, Tomanek, \& Verleger, 2008; Kiesel et 
al., 2008; Lleras \& Enns, 2004, 2005, 2006; Verleger et al., 2004, Verleger, Görgen, \& Jaśkowski, 2005). In contrast, also a single mechanism could theoretically explain these empirical findings when assuming that the effectiveness of this mechanism is altered by the mask structure such that it produces larger effects with relevant masks (Verleger, \& Jaśkowski, 2007).

A useful experimental tool to assess how and where inverse priming originates with both types of masks is the non-motor or cue-priming paradigm (Mattler, 2006, 2007).

\section{Cue priming}

Cue-priming is a straightforward extension of the traditional masked priming paradigm. Compared to it, the sequence of prime, mask and the target stimulus remains the same. However, the third stimulus now serves as a cue indicating which of two tasks has to be performed on a subsequent target stimulus. Thus, in inverse cue-priming four stimuli are presented in succession. The cue can be preceded by a prime which signals either the same (congruent case) or the opposite task (incongruent case). Consequently, cue-priming can be understood as a transfer of target-priming to priming of more complex cognitive operations (cf. Mattler, 2003). In contrast to target priming, the cue stimulus does not require a response but the execution of certain cognitive operations related to it. From this perspective, the processes that lead to target- and cue-priming could be the same only that in cue-priming the cue requires a more complex "response".

For instance, Mattler (2007) introduced a task switching variant in which the cue provided information about the dimension (instrument vs. pitch) along which an auditory target stimulus had to be classified. An inverse cue-priming effect with better performance on incongruent trials as compared to congruent trials was obtained. The effect exhibited a similar time-course as target-priming: inverse effects increased with increasing stimulus onset asynchrony between mask and cue (MC-SOA), which corresponds to the mask-target SOA in inverse target-priming.

Apart from the comparable time-course, inverse cue-priming effects with relevant masks turned out to be independent of prime-visibility just like inverse target-priming effects (Mattler, 2007). Additionally, Mattler (2006) compared the influence of perceptually similar and dissimilar congruent primes with incongruent primes in inverse target- and cue-priming 
when relevant masks were applied. For both, target- and cue-priming, only perceptual similarity modulated performance: if prime and target or cue-stimuli, respectively, were perceptually similar performance was worse as compared to perceptually dissimilar but congruent stimuli. In contrast, incongruent stimuli did not further impair performance. These results point to a perceptual source of both, inverse cue- and target priming effects.

These commonalities suggest a common mechanism underlying target- and cue-priming. Therefore, cue-priming seems to be suited to gain new insights into the generation of inverse priming, especially because, in cue-priming, neither the prime nor the cue stimulus is related to any motor consequences. Instead both stimuli are assigned to a certain task. For this reason, processes that result in inverse cue-priming effects cannot be located at the motor stage. Therefore, the current evidence for inverse cue-priming points to a premotor-locus of inverse priming which is in line with previous results (Mattler, 2006). However, these results might be due to the relevant metacontrast-masks that have been employed. Under these conditions, Object Updating might be responsible for inverse cue-priming to occur. Until now, inverse cue-priming has not been demonstrated when irrelevant masks are used. Inverse cue-priming with irrelevant masks would provide clear evidence against a motor locus of the mechanism being involved with the generation of inverse priming with such irrelevant masks. If the processes caused by the mask that lead to inverse priming operate on a pre-motor level then cue-priming with irrelevant masks would be expected. On the other hand, if motor inhibition is responsible for the reversal no priming effect could arise because in cue-priming primes are independent of motor preparation processes.

\section{Overview}

We performed three experiments using a new cue-priming task. While target stimuli and the related cue-priming tasks remained the same across all experiments, primes, masks and cues differed (see Figure 1). Experiment 1 employed relevant masks extending previous demonstrations of inverse cue-priming to the new paradigm introduced here. In contrast, Experiment 2 and 3 used irrelevant masks. We expected to replicate inverse cue-priming in Experiment 1 . However, this effect might be completely due to the use of a relevant mask. To the extent that we also observe inverse cue-priming effects in Experiment 2 and 3 we sought to conclude that these effects originate from a premotor locus also with irrelevant masks. 
Please insert Figure 1 about here

\section{Experiment 1: Cue-Priming with relevant masks}

Experiment 1 is directly linked to the cue-priming experiments carried out by Mattler (2007). We extended his paradigm by introducing a new cue-priming task. Here, pictures composed of photographs of a human face overlaid with a visual scene served as response targets. Depending on the prior cue, subjects had to attend either to the gender of the face or the location of the visual scene. Like Mattler (2007), we employed relevant metacontrast-masks to provide optimal conditions for inverse cue-priming to occur.

Method

Participants. 12 psychology students from the University of Goettingen (6 male, mean age 22.4 years, range 19-27 years) participated in the experiment. 11 were right-handed by selfreport, and all had normal or corrected- to-normal vision. Each participant received $7 €$ per hour for participation.

Tasks. Subjects had to perform two different tasks across four separate sessions. (a) Choice $R T$ task: During the first three sessions, subjects responded to a picture which consisted of a human face superimposed with a visual scene. A preceding cue stimulus indicated consistently whether the subjects had to judge the gender of the face (cue = diamond) or the location of the visual-scene (cue $=$ square). A female face required responding with the left index finger, a male face with the right index finger; in the same way, an outdoor-scene required responding with the left index finger, an indoor-scene with the right index finger. (b) Prime recognition task: In the final session, a prime recognition task was implemented. After being informed about the presence of the prime stimuli, subjects had to report the shape of the primes without speed stress in order to obtain a measure of prime visibility. A diamondshaped prime required a response with the left index finger, a square-shaped prime with the right index finger.

Stimuli. The stimulus sequence was held constant across the four sessions (see Figure 1). Apart from their size, primes were identical to the cue stimuli and consisted of either 
diamond-shaped or square-shaped filled black forms. Primes and cues were of the same shape (congruent trials) in half of the trials and otherwise of different shapes (incongruent trials). The metacontrast-mask was built by superimposing both cue-stimuli. Both prime alternatives fitted into its inner cut-out (with a gap of 1 pixel). The target stimuli were composed of two transparently superimposed photographs showing the face of a person and a visual scene. Male faces were obtained from the CVL Face Database (Solina et al., 2003; http://lrv.fri.unilj.si/facedb.html), female faces were self-made photographs from volunteers that gave written permission for scientific usage and publication. All stimuli were presented in black on white on the computer monitor. Stimuli were positioned at a fixation cross in the centre of the monitor. Squares and diamonds were made of the same number of pixels. Prime squares and diamonds subtended visual angles of about $1.1^{\circ}$ and $1.6^{\circ}$, respectively. The outer contour of the square and diamond cues subtended about $1.8^{\circ}$ and $2.6^{\circ}$ of visual angle, respectively. The target stimuli subtended about $10.3^{\circ}$ in the horizontal dimension and $12.3^{\circ}$ in the vertical. During all sessions, incorrect responses were signalled by visual error feedback (the german word for error: "Fehler").

Procedure. Participants were tested individually on separate days. At the beginning, they gave written informed consent. Participation depended on performance in each session assessed by a performance index which was a weighted mean of the overall RT and error rate in that session. Missing a given performance criterion resulted in exclusion from further participation. Participation depended on performance in order to ensure high motivation and meeting task requirements. However, all participants met the criterion.

During the experiment, participants sat in a dimly lit room $55 \mathrm{~cm}$ in front of a computer monitor with the chin fixed in a rest. The first and the fourth session started with instructions and a practice section. In the first session, participants were initially familiarized with the two picture discrimination tasks. To this end, they classified 16 pictures presented alone according to the person's face and 16 trials to the visual scene. Then, they were introduced to the meaning of the cue and performed 36 practice trials under equal conditions as in the experiment. Instructions emphasized the importance of the active preparation of the task signalled by the cue before target onset. To remind them of this aspect, participants were asked after each block of trials, to report how well they could prepare for the indicated task. At the beginning of the fourth session, participants were informed about the presence of the prime. They were shown the entire stimulus sequence with long stimulus durations and they performed some practice trials. This procedure ensured that participants understood the task 
and recognized the perceptual effects that were generated by the stimulus sequence. They were instructed to identify the primes as accurate as possible without speed stress.

Stimuli and timing parameters were identical across the second, third and fourth session. The initial practice session only differed with respect to the cue-target interval which was successively shortened during this session. From its initial value of $600 \mathrm{~ms}$ it was reduced in steps of $200 \mathrm{~ms}$ each time after three blocks until reaching $50 \mathrm{~ms}$ in the $12^{\text {th }}$ block, henceforward it was held constant during the entire experiment. Trials started with a fixation cross. After $650 \mathrm{~ms}$ the prime was presented for $17 \mathrm{~ms}$, followed by the mask with a SOA of $67 \mathrm{~ms}$. Mask duration was $33 \mathrm{~ms}$ und cue duration $100 \mathrm{~ms}$. The mask-cue SOA (MC-SOA) was either $0,50,100,150$ or $200 \mathrm{~ms}$. With 0 -ms-MC-SOA the mask was absent and thus the cue followed the prime immediately. The target followed the cue with a SOA of $150 \mathrm{~ms}$ and was presented for $700 \mathrm{~ms}$. Then, the fixation cross reappeared on the screen until a response was recorded. The computer waited for $2500 \mathrm{~ms}$ after target-onset for the response. In the fourth session, the computer did not proceed until a response was received. In all sessions, the next trial started after the response with a variable inter-trial-interval ranging from $1250 \mathrm{~ms}$ to $2050 \mathrm{~ms}$ (pseudo-exponential distribution).

Apparatus. The experiment was run on a personal computer with a CRT-display $(60 \mathrm{~Hz}$, resolution 1024*768). Participants delivered their responses by pressing CTRL-buttons on the computer keyboard with their index fingers.

Design. Response speed and accuracy were the dependent variables in the choice RT task with independent variables MC-SOA (0, 50, 100, 150, $200 \mathrm{~ms}$ ), and Congruency (congruent vs. incongruent). Only data from the second and third session were included in this analysis. In the prime identification task of the fourth session prime visibility was assessed by accuracy rates as dependent measure. In each session, data from 14 experimental blocks of 40 trials each were included in the analysis. Consequently, 112 replications were achieved for each of the 10 conditions resulting from the factorial combination of MC-SOA and Congruency. The estimation of prime visibility was based on 560 trials. Within each session the order of the trials was randomized.

Statistical analysis. RTs were summarized by trimmed means for each participant and condition. Error and post-error trials were excluded. RTs and the arcsine-transformed errorrates for the two tasks were analyzed separately with a two-way repeated-measures analysis of variance (ANOVA) with the within-subject factors MC-SOA and Congruency. For each 
dependent variable two separate ANOVAs were performed: One including all levels of SOA, one including only those levels that are supposed to lead to inverse priming, i.e. $50,100,150$ and $200 \mathrm{~ms}$ MC-SOA. Prime recognition performance was assessed by signal detection methods with d' serving as a global measure of prime visibility. Arbitrarily, we defined the square as signal, and counted a correct response to a square as "hit" and an incorrect response to a diamond as "false alarm". Measures of d' were determined for each cue stimulus and each MC-SOA separately, and then averaged across these conditions.

Results

Accuracy data. Results are depicted in Figure 2A. Errors occurred on $4.4 \%$ of the trials. Error rates were not affected by any experimental manipulation irrespective of whether all SOAs were considered jointly or only non-zero SOAs (50, 100, 150, $200 \mathrm{~ms})$.

$R T$. As expected, priming effects depended on the MC-SOA when considering the entire SOA range as reflected by the significant interaction of Congruency and SOA, F = 4.42, p $<.01$; MSE $=122.0$. At the 0 -ms-SOA, positive priming was observed with faster responses on congruent trials $(517 \mathrm{~ms})$ as compared to incongruent trials $(526 \mathrm{~ms}), \mathrm{t}(11)=2.7, \mathrm{p}<.05$. This effect reversed for non-zero MC-SOAs $(-11,-15,-11,-9 \mathrm{~ms}, \mathrm{t}(11)=-2.0,-2.8,-4.0,-1.5$, $\mathrm{p}=.08, .02, .002, .17$ for the $50,100,150$ and $200 \mathrm{~ms}$ SOA, respectively). Probably due to the preponderance of conditions supporting the occurrence of inverse priming, also the main effect of Congruency reached significance, $\mathrm{F}=7.64, \mathrm{p}<.05 ; \mathrm{MSE}=205.4$ (-7 ms). Restricting the analysis to non-zero SOA values complemented this picture: while there was no interaction of SOA and Congruency, $\mathrm{F}<1$, incongruent trials led to faster responses (511 $\mathrm{ms})$ as compared to congruent trials $(522 \mathrm{~ms}), \mathrm{F}=18.38, \mathrm{p}<.01 ; \mathrm{MSE}=168.77$. Furthermore, response speed depended on SOA, $\mathrm{F}=3.3, \mathrm{p}<.05 ; \mathrm{MSE}=315.5$.

Please insert Figure 2 about here

Prime recognition. Overall, the prime was correctly classified on $58 \%$ of all trials which corresponds to an average $d^{\prime}=.31$. The MC-SOA did not affect prime recognition performance, $\mathrm{F}(4,44)<1$ with means of $.38, .28, .22, .41, .30$ for MC-SOA 0, 50, 100, 150, 
$200 \mathrm{~ms}$. Post-hoc t-tests demonstrated that the performance deviated significantly from chance level for SOA 0, 50, 150 and $200 \mathrm{~ms}$, all $\mathrm{t}(11)>2.65 ; \mathrm{p}<.05$, while it missed significance at the 100-ms-SOA, $\mathrm{t}(11),=1.85, \mathrm{p}=.09$.

To examine a possible relationship between the size of the priming effects and prime visibility, we computed correlations for each SOA separately. Neither RT-priming effects $(|\mathrm{r}|$ $<.25, \mathrm{p}>.42)$ nor priming effects on error rates $(|\mathrm{r}|<.41, \mathrm{p}>.19)$ correlated significantly with prime recognition performance. However, the absence of a linear relationship between prime visibility data and priming effects as reflected in the Pearson correlation coefficient does not rule out a different relationship. Therefore, we analysed priming effects separately for those subjects showing best versus worst prime recognition performance (median-split, see Figure 2B). In the subgroup of those subjects with the best recognition performance the average d' was .51 (ranging from .40 to .69 across MC-SOAs) which is significantly better than chance level, all $\mathrm{t}(11)>2.80, \mathrm{p}<.05$, except for the 150 -ms-SOA condition, $\mathrm{t}(11)=1.90, \mathrm{p}=.12$. In the second subgroup the average d' was .12 (ranging from -.06 to .29 across MC-SOAs). ttests did not indicate above-chance performance, $\mathrm{t}(11)<1.33, \mathrm{p}>.24$, in this subgroup except for the $150-\mathrm{ms}-\mathrm{SOA}, \mathrm{t}(11)=4.48, \mathrm{p}<.01$. Having established two extreme groups which differ with respect to their capacity to recognize the primes, we next focused on potential difference in priming effects among the two groups. However, a mixed ANOVA including the factors Visibility Group, Congruency and SOA revealed no difference with respect to priming effects on both, RTs: $\mathrm{F}(1,10)=1.52 ; \mathrm{p}=.25 ; \mathrm{MSE}=56271$, as well as accuracy data, $\mathrm{F}(1,10)$ $<1)$. Even the three-way interaction of Visibility Group, Congruency and SOA failed to reach significance for RT data, $\mathrm{F}(4,40)<1$, and accuracy data, $\mathrm{F}(4,40)=1.35 ; \mathrm{p}=.27$; $\mathrm{MSE}=$ .00056. Thus, even though subjects substantially differed with respect to their ability to consciously perceive the primes, priming effects on the Choice RT task were the same.

\section{Discussion}

Experiment 1 demonstrated inverse cue-priming effects with a new cue-priming paradigm. Thus, findings extend the generality of inverse non-motor priming which has been demonstrated before with relevant masks for two different paradigms (Mattler, 2007). In accordance with these earlier demonstrations of inverse cue-priming, priming effects were again independent of prime visibility conflicting with the view that effective masking is the crucial prerequisite for inverse priming (e.g. Schlaghecken \& Eimer, 2002). Inverse cue- 
priming emerged also within those subjects showing prime recognition performance above chance-level and its time-course was indistinguishable from that of another subgroup of participants showing chance-level performance in the prime recognition task. This comparison across participants is in line with several recent studies demonstrating inverse priming with completely unmasked primes (Jaśkowski, 2007; 2008; Jaśkowski, et al., 2008; Lleras, \& Enns, 2006). These results suggest that prime visibility is not causally related to the generation of inverse priming which seems to be widely accepted now (cf. Schlaghecken, Blagrove, \& Maylor, 2008; Sumner, 2007)

The existence of inverse non-motor priming with relevant masks suggests that the mechanism producing this effect is not located at motor levels of processing because the prime is not linked to any motor consequences in the cue-priming paradigm and thus it cannot exert its influence on a motor level. The current results, thus, accord well with evidence from two further studies which locate inverse priming at perceptual levels of processing (Mattler, 2006; Krüger, Klapötke, \& Mattler, 2011). On the other hand, the results are at odds with current theoretical accounts on inverse priming locating the effect at motor levels of processing (Jaśkowski, \& Przekoracka-Krwczyk, 2005; Lleras \& Enns, 2006; Schlaghecken, \& Eimer, 2002). However, as outlined in the introduction, inverse priming with relevant masks might completely result from Object Updating. Using irrelevant masks, inverse priming might be generated in a different way, potentially via mask induced motor inhibition of the primed response. If this is true and cue-priming indeed relies on the same mechanism as target priming, which is suggested by several analogous results for both effects (Mattler, 2006, 2007), inverse cue-priming should not exist with irrelevant masks. This hypothesis was examined in Experiment 2.

\section{Experiment 2: Cue Priming with irrelevant masks}

Experiment 2 served to demonstrate the existence of inverse non-motor priming when irrelevant masks are used. To achieve a substantial reduction of prime visibility we used a pattern masking procedure which has been successfully employed by several earlier studies on inverse priming (e.g. Schlaghecken, \& Eimer, 2006). The cues were double arrows signalling which task has to be performed on the target stimulus. Primes were identical to the cues and were masked by a complex pattern of random horizontally and vertically oriented lines (see Figure 1). Thus, these random line masks do not comprise any task-relevant features 
and are therefore irrelevant masks. With such irrelevant masks inverse priming cannot result from the perceptual interaction of prime and mask.

\section{Method}

Participants. 24 new students from the University of Goettingen (12 male, mean age 24.2 years, range 19-23 years) participated in this experiment. All but one were right-handed by self-report and all had normal or corrected-to-normal vision. Furthermore, three participants did not complete the experiment, two of them because they missed the performance criterion in one of the sessions (see procedure of Experiment 1). The other one dropped out voluntarily. Each participant received $7 €$ per hour for participation.

Stimuli. The stimulus sequence was comparable to that of Experiment 1. Apart from the target stimuli new stimuli were created. Primes and cue stimuli were identical and consisted in a left or right-pointing double arrow $(<<$ or $>>)$ which possessed the same orientation on half of the trials (congruent condition) and different orientations otherwise (incongruent condition). Masks were a pattern of 130 horizontally and vertically aligned lines of varying length (from ca. $0.06^{\circ}$ to $0.45^{\circ}$ of visual angle) and width (from ca. $0.08^{\circ}$ to $0.16^{\circ}$ ). To achieve optimal masking, a different randomly generated mask was presented on each trial (cf. Schlaghecken, et al., 2008). The individual lines were centred on a $13 \times 10$ grid which subtended about $1.7^{\circ}$ $\mathrm{x} 1.4^{\circ}$ of visual angle. Primes and cues subtended $1.5^{\circ} \times 1.0^{\circ}$.

Procedure. Again, four sessions were performed on separate days with the initial session serving to practice the choice RT task in which the cue-target interval was successively reduced. In the fourth session prime recognition was measured. To ensure optimal masking, timing parameters deviated slightly from those of Experiment 1. Primes were now presented for $20 \mathrm{~ms}$; masks followed with a SOA of $30 \mathrm{~ms}$ and were presented for $100 \mathrm{~ms}$. The MCSOA varied between $0,100,150$ or $200 \mathrm{~ms}$ and the cue was presented for $100 \mathrm{~ms}$. The $50 \mathrm{~ms}-$ MC-SOA condition was omitted to avoid overlapping presentation of mask and cue. Again, in the 0 -ms-SOA condition no mask was presented. $150 \mathrm{~ms}$ after cue onset the target was presented for $700 \mathrm{~ms}$.

Design. Dependent and independent variables were identical to Experiment 1. However, in this experiment the MC-SOA varied on only four levels (0, 100, 150, $200 \mathrm{~ms})$. Choice-RT data from 1280 trials from the second and third session were included in the analysis. Every 
session now comprised 20 blocks with 32 trials each. Thus, 160 replications were achieved for each of the eight conditions. Prime visibility was measured on the basis of 640 trials from the fourth session.

Tasks, Apparatus, Statistical analysis. See Experiment 1.

Results

Accuracy data. Results are depicted in Figure 3A. Errors occurred on $7.0 \%$ of all trials. As expected, when all levels of SOA were included in the analysis no effect of Congruency could be observed, $\mathrm{F}(1,23)<1$, but Congruency interacted with SOA, $\mathrm{F}(3,69)=5.03, \mathrm{p}<.01$, MSE $=.00057$. Priming effects were positive at the 0 -ms-SOA $(+1.8 \%, \mathrm{t}(23)=2.4, \mathrm{p}<.05)$ while they tended to be reversed for the other SOA conditions $(-1.2 \%,-0.4 \%$ and $-1.6 \%, \mathrm{t}(23)=-$ $1.5,-0.6,-2.7, \mathrm{p}=.15, .57, .01$, respectively). The absolute error rates did not depend on the MC-SOA, F $<1$. When the 0 -ms-SOA condition was excluded from the analysis, the reported numerical trend towards inverse priming failed to reach significance, $\mathrm{F}(1,23)=3.4, \mathrm{p}=.08$, MSE $=.0013$. No other effects did not became significant.

Please insert Figure 3 about here

$R T$. Most important, RT priming effects depended on SOA, $\mathrm{F}(3,69)=76.8, \mathrm{p}<.00001$, MSE $=511$, with a large positive effect at the shortest SOA $(+105 \mathrm{~ms}, \mathrm{t}(23)=9.0, \mathrm{p}<.0001)$ and a trend for inverse effects for the other SOA conditions $(-4 \mathrm{~ms},-13 \mathrm{~ms}$ and $-8 \mathrm{~ms}, \mathrm{t}(23)=-0.6$, $2.5,-1.2, \mathrm{p}=.55, .02, .24$, respectively). Additionally, RT depended on SOA, F(3,69) = 18.1, $\mathrm{p}<.00001, \mathrm{MSE}=630$, increasing SOA was accompanied by monotonically decreasing RTs $(663 \mathrm{~ms}, 661 \mathrm{~ms}, 642 \mathrm{~ms}$, and $631 \mathrm{~ms})$. Seemingly due to the large positive priming effect at the 0-ms-SOA condition, the overall Congruency effect also reached significance, $\mathrm{F}(1,23)=$ $11.85, \mathrm{p}<.01, \mathrm{MSE}=1597$. Again, when considering only those conditions with the mask included, the Congruency effect failed to reach significance, $\mathrm{F}(1,23)=2.7, \mathrm{p}=.12$, MSE $=$ 1015.8 and its interaction with SOA as well, $\mathrm{F}(2,46)=1.1, \mathrm{p}=.33, \mathrm{MSE}=229.6$. Only the main effect of SOA still became significant, $\mathrm{F}(2,46)=25.9, \mathrm{p}<.00001, \mathrm{MSE}=425.2$. 
Spatial Compatibility. Double arrows were used as primes and cues. It has been assumed that arrow stimuli are able to automatically invoke corresponding motor activation (Eimer, 1995) which could interact with the responses required by the target stimuli. When cue orientation and response required by the target are compatible, performance benefits could occur while a misleading cue could yield performance costs. To examine the role of spatial compatibility, we performed a repeated-measures ANOVA including the spatial compatibility of cue and response as additional factor. Subjects tended to respond faster when the cue orientation matched the correct response $(632 \mathrm{~ms})$ as compared to the incompatible condition (648 $\mathrm{ms})$, $\mathrm{F}(1,23)=3.76, \mathrm{p}=.06, \mathrm{MSE}=6474$. When restricting the analysis to those SOAs producing inverse priming, it became apparent that spatial compatibility altered inverse priming effects as indexed by the two-way interaction of these factors, $\mathrm{F}(1,23)=4.66, \mathrm{p}<.05, \mathrm{MSE}=208.4$. Inverse priming effects were about twice as large for compatible trials ( $-12 \mathrm{~ms}$ ) as compared to incompatible ones $(-5 \mathrm{~ms})$. Other effects failed to reach significance. In particular, priming effects on accuracy data were unaffected by spatial compatibility, $\mathrm{F}<1$.

Prime recognition. Masked primes were correctly identified on $58 \%$ of all trials (corresponding to $\mathrm{d}^{\prime}=.32$ ) and on $94 \%$ of all trials $\left(\mathrm{d}^{\prime}=2.06\right)$ when the cue followed the prime immediately. The effect of omitting the mask on prime recognition is reflected by the significant main effect of SOA, F(3,69) = 138.4, p <.00001, MSE $=.131$, whereas SOA did not affect prime recognition performance when only considering those conditions including a mask, $\mathrm{F}<1$. At all levels of SOA, prime recognition performance differed significantly from chance level $(\mathrm{t}(23)>2.61, \mathrm{p}<.02)$. To examine whether prime visibility was related to priming effects we computed correlations. In fact, differences with respect to individual prime visibility were accompanied by differences with respect to inverse priming effects as indexed by a significant correlation of RT-priming effects at all non-zero SOAs: $r=.44, r=.48, r=$ .64 (all $\mathrm{p}<.04)$. Such a relationship was absent in the accuracy data $(r<.37, \mathrm{p}>.07)$.

Next, relations between inverse priming effects and prime recognition performance were explored in a group analysis. Because more data was acquired compared to Experiment 1, we split the data into three equal sized subgroups according to the proportion of correctly classified masked primes: one group with best, one with worst and one with intermediate recognition performance. We then compared prime recognition performance in the two extreme groups (see Figure 3B). In the group with best performance the prime identification rates exceeded chance level at each $\mathrm{SOA}, \mathrm{t}(7)>5.28, \mathrm{p}<.002\left(\mathrm{~d}^{\prime}=.92, .85, .86\right.$, 
respectively), while performance was at chance level for the poor performance group, $|\mathrm{t}(7)|<$ $2.4, \mathrm{p}>.05 ; \mathrm{d}^{\prime}=.07,-.15,-.07$, respectively).

In order to examine inverse priming within these two extreme subgroups, choice-RT data (only 100, $150200 \mathrm{~ms}$ SOA conditions) was subjected to a mixed ANOVA with the withinsubject factors Congruency and SOA and the between-subject factor Visibility Group. Inverse priming effects on RTs differed between the groups, Visibility Group x Congruency: $F(1,14)$ $=7.27, \mathrm{p}<.02, \mathrm{MSE}=906.9$, irrespective of the SOA, Visibility Group $\mathrm{x}$ Congruency $\mathrm{x}$ SOA: $\mathrm{F}<1$. Effects were more pronounced within the poor performance group $(-23 \mathrm{~ms})$ as compared to the best performance group $(10 \mathrm{~ms})$. Error rate effects also differed between the groups, Visibility Group x Congruency: $\mathrm{F}(1,14)=5.05, \mathrm{p}<.05$, MSE $=.0012$, while the timecourse of priming effects was the same, Visibility Group x Congruency x SOA: F < 1 . The inverse priming effect on error rates was more pronounced for the worst performance group ($3.3 \%)$ as compared to the best performance group (-0.2\%).

Thus, not only the individual ability to perceive the primes differed but priming effects were also affected. To explore the reliability of the reported numerical trends towards inverse priming among the poor performance group, we subjected data from each group separately to a repeated-measures ANOVA including the factors Congruency and SOA. In the poor performance group, Congruency actually influenced RTs, F(1,7) = 14.03, p <.01, MSE = 458.7, and error rates, $\mathrm{F}(1,7)=7.23, \mathrm{p}<.04, \mathrm{MSE}=.0018$. In contrast, in the good performance group, Congruency neither affected RT nor error rate performance, both $\mathrm{F}<1$.

\section{Discussion}

Experiment 2 provided evidence for inverse priming with irrelevant masks. In contrast to Experiment 1, however, inverse cue-priming effects were insignificant when the entire sample of participants was considered. Instead, when restricting the analysis to those subjects showing chance-level performance on the prime recognition task, significant inverse priming effects were found. Such a link between prime visibility and priming effects has been reported in several previous studies on inverse priming. For example, Eimer and Schlaghecken (2002) manipulated prime visibility by either varying prime duration or mask density. Individual prime identification thresholds were determined by an adaptive staircase procedure. They observed a remarkable correspondence between the individual transition point from subliminal to supraliminal prime perception and the direction of priming effects: subliminal 
primes elicited inverse priming, supraliminal ones had positive priming effects. Sumner, Tsai, Yu, \& Nachev (2006) also manipulated prime duration and observed that well visible primes of long durations produced positive effects and inverse priming resulted from the use of subliminal primes of short duration. Comparing letter masks of different masking efficiency, Klapp (2005) also found larger inverse priming effects for the more effective masks (see also Klapp, \& Hinkley, 2002). These studies, thus, altered prime visibility by the experimental manipulation of some factor and studied the consequences on priming effects. In addition, a trend towards larger inverse effects of primes when masking is more effective has also been observed when relating inter-individual differences in prime recognition performance to priming effects (Sumner, 2007). Taken together, it is not surprising that we also found such a relation. But interestingly, no such dependence was observed in Experiment 1 when relevant masks were used. This issue will be further treated in the General Discussion.

Inverse priming effects were modulated by the spatial compatibility of the cue stimulus which was a double arrow and the response required by the target. Effects were more pronounced when the orientation of the cue matched the correct response to the target. Thus, cue orientation seemed to affect task performance even though the cues did not possess any predictive value with respect to the response required by the target. Cue orientation matched the response on $50 \%$ of all trials and conflicted with it on the remaining trials. Previous research demonstrated an automatic response activation of arrow stimuli as reflected in lateralized readiness potentials even though these stimuli were unpredictive or even detrimental in the studied task context (Eimer, 1993; 1995). Further support for the automatic processing of unpredictive arrow cues comes from studies on reflexive attentional shifts (Kuhn, \& Kingstone, 2009; Pratt, Radulescu, Guo, \& Hommel, 2010; Tipples, 2008) which are supposed to invoke corresponding motor preparation processes (Rizzolatti, Riggio, Dascola, \& Umiltà, 1987). To avoid interference effects of potential motor activations resulting from automatic cue processing, we replicated the experiment with symmetrical cues which cannot be compatibly mapped onto the motor responses.

\section{Experiment 3: Inverse Cue-Priming with irrelevant masks and symmetrical cues}

Experiment 3 was an exact replication of Experiment 2 except for the design of prime and cue stimuli. We created stimuli that were neutral with respect to the spatial arrangement of 
response buttons to avoid any spatial compatibility effects between cue-orientation and the target response which might interfere, and therefore reduce inverse priming effects.

Method

Participants. 24 new psychology students from the University of Goettingen (6 male, mean age years, range 19-23 years) participated in this experiment. All but one were right-handed by self-report and all had normal or corrected-to-normal vision. All participants completed the experiment. Each participant received $7 €$ per hour for participation.

Stimuli. Masking and target stimuli remained the same compared with Experiment 2. The prime and cue stimuli were modified yielding two patterns composed of two vertically aligned arrows pointing in opposite directions (see Figure 1). One variant consisted of two arrows pointing towards each other which required the subject to perform the face discrimination task. The alternative consisted of two arrows pointing away from each other requiring the subject to perform the scene task. Both stimuli resulted from rearrangement of the elements forming the double arrow stimuli in order to keep mask relevance comparable to Experiment 2.

Tasks, Procedure, Apparatus, Deign, Statistical analysis. See Experiment 2.

Results

Accuracy data. Results are depicted in Figure 4A. The overall error rate was $5.2 \%$. When all SOAs were included in the analysis, no effect reached significance. However, when restricting the analysis to non-zero SOAs, a small but reliable inverse priming effect became apparent, $\mathrm{F}(1,23)=10.03, \mathrm{p}<.005$, with fewer errors on incongruent trials $(4.7 \%)$ as compared to congruent trials $(5.2 \%)$.

Please insert Figure 4 about here

$R T$. RTs were affected by Congruency. Averaging across SOAs, subjects responded faster on congruent $(535 \mathrm{~ms})$ than incongruent trials $(547 \mathrm{~ms}), \mathrm{F}(1,23)=46.30, \mathrm{p}<.00001, \mathrm{MSE}=$ 141.2. This overall tendency towards positive priming was due to a large positive RT-priming 
effect in the 0 -ms-SOA condition $(+116 \mathrm{~ms}, \mathrm{t}(23)=8.1, \mathrm{p}<.0001)$, while no priming effects were found for the other SOAs $(+1,-4 \mathrm{~ms},-2 \mathrm{~ms},|\mathrm{t}(23)|<1.6, \mathrm{p}>.14)$. This time course of priming effects is reflected in a significant interaction of Congruency and SOA, F $(3,69)=$ 43.30, $\mathrm{p}<.00001, \mathrm{MSE}=196.2$. Additionally, SOA affected response times, $\mathrm{F}(3,69)=19.03$, $\mathrm{p}<.00001, \mathrm{MSE}=279.6$ (means 548, 551, 535, $529 \mathrm{~ms}$ for the respective SOAs). In contrast to accuracy data, inverse priming effects in the non-zero SOA conditions were far from being significant when considered alone, $\mathrm{F}<1$, and their size was not modulated by the SOA, $\mathrm{F}(2,46)=1.11, \mathrm{p}=.34, \mathrm{MSE}=56.92$. Still, the main effect of SOA reached significance, $\mathrm{F}(2,46)=39.54, \mathrm{p}<.000001, \mathrm{MSE}=157.9$.

Prime recognition. Primes were correctly identified on $55.7 \%$ of the trials $\left(d^{\prime}=.19\right)$ in which they were masked. Unmasked primes presented in the 0-ms-SOA condition were recognized on $93.0 \%$ of the trials $\left(d^{\prime}=1.91\right)$. Thus, SOA modulated prime recognition performance, $\mathrm{F}(3,69)=291.1, \mathrm{p}<.00001, \mathrm{MSE}=.062$. However, the MC-SOA had no effect when only the masked trials were considered, $\mathrm{F}<1$, (mean performance 54.8, 56.3, 56.1\% for the respective SOAs.). Prime recognition performance differed significantly from chance level for all SOA conditions, $\mathrm{t}(23)>2.6, \mathrm{p}<.02$.

Then, we considered the relation between priming effects and individual prime recognition performance (see Figure 4B). In a first approach, we explored correlations for each SOA. For priming effects on RTs no significant correlations were found for any SOA $(|\mathrm{r}|<.27, \mathrm{p}>.19)$ but the $150-\mathrm{ms}-\mathrm{SOA}$ condition $(\mathrm{r}=.41, \mathrm{p}<.05)$. Correlations for accuracy data also failed to reach significance $(|\mathrm{r}|<.23, \mathrm{p}>.25)$. Then, we again sorted participants according to their prime recognition performance into three groups of equal size and compared priming effects among those with best and worst recognition performance. While performance was at chance level in the poor performance group, $|\mathrm{t}(7)|<1.3, \mathrm{p}>.23$, d' $=-.04,-.02, .03$, respectively, it was clearly above chance level in the good performance group, $t(7)>2.95, p<.03$, d' $=.39$, $.47, .50$.

In order to test the dependency of priming effects on prime recognition performance choiceRT data was subjected to an omnibus-ANOVA including the factors Visibility Group, Congruency and SOA (only non-zero values). Neither the size of RT-priming effects differed between the groups, Visibility Group x Congruency: $\mathrm{F}<1$, nor the priming effects on error rates, $\mathrm{F}<1$. However, the three-way interaction of Visibility Group, Congruency and SOA just missed significance, at least for RT data: $F(2,28)=3.16, p=.06, \mathrm{MSE}=40.1$, but not for accuracy data, $\mathrm{F}<1$. 
To test the reliability of priming effects within the groups, we performed two separate twoway repeated-measures ANOVAs with the factors Congruency and SOA. In the good performance group no interaction of Congruency with SOA was obtained, neither for RT-data nor for accuracy data, both $\mathrm{F}<1$. Congruency did not affect response times, $\mathrm{F}<1$, but error rates, $\mathrm{F}(1,7)<11.31, \mathrm{p}<.02, \mathrm{MSE}=.000039$, with fewer errors on incongruent trials $(4.8 \%)$ as compared to congruent trials $(5.4 \%)$. In the poor performance group, an interaction of Congruency and SOA indicated that the size of priming effects on RTs depended on the SOA, $\mathrm{F}(2,14)=4.26, \mathrm{p}<.05, \mathrm{MSE}=49.74$. Post-hoc testing revealed a significant inverse priming effect at the $150 \mathrm{~ms}-\mathrm{SOA}, \mathrm{t}(7)=-2.51, \mathrm{p}<.05$. The interaction was absent in the accuracy data, $\mathrm{F}<1$.

\section{Discussion}

In Experiment 3, we substituted the double arrow stimuli which were employed as primes and cues in Experiment 2 in order to rule out an interference of automatic motor consequences of these stimuli with the task. Contrary to our expectations, however, inverse priming effects were even less pronounced in Experiment 3. While we found an SOA independent inverse priming effect on error rates irrespective of prime recognition performance, RTs were unaffected by prime-cue congruency. Only when restricting the analysis to subjects being unable to perceive the prime, a small but significant effect in the 150-ms-SOA condition could be detected.

\section{General Discussion}

Experiment 1 replicated inverse cue-priming with relevant masks with a new cue-priming task comprising complex visual stimuli which adds further evidence for the generality of the phenomenon (cf. Mattler, 2007). In line with previous findings, inverse cue-priming with such relevant metacontrast masks turned out to be independent of prime visibility (Mattler, 2007). Beyond this, the results from Experiment 2 and 3 provide evidence for inverse cue-priming when irrelevant masks are used. Prime visibility seemed to be closely linked to priming effects. Evidence for inverse cue-priming with irrelevant masks was only obtained within those subjects being unable to perceive the prime which is in line with previous findings in the domain of target priming (Eimer, \& Schlaghecken, 2002; Klapp, 2005; Sumner, 2007). 
Importantly, evidence for inverse cue-priming with irrelevant masks improves our understanding of the mechanism underlying inverse priming. While the effect could completely result from simple perceptual prime-mask interactions when relevant masks are used (Lleras, \& Enns, 2004), such an explanation is ruled out with irrelevant masks. If both, target and cue-priming are generated by a common mechanism (Mattler, 2007), this mechanism cannot be located at motor levels of processing which conflicts with the majority of theoretical accounts on inverse priming (Lleras \& Enns, 2006; Jaśkowski \& PrzekorackaKrawczyk, 2005; Schlaghecken, \& Eimer, 2002). Potential alternatives are discussed in the following section.

The locus of inverse priming effects

A large body of research provided evidence for the notion that inverse priming does not originate from a single source (Klapp, 2005; Sumner, 2007). It is now widely accepted that with relevant masks, prime-induced saliency modulations of the mask elements contribute to inverse priming (Jaśkowski, \& Przekoracka-Krawczyk, 2005; Kiesel, Berner, \& Kunde, 2008; Lleras, \& Enns, 2006; Schlaghecken, \& Eimer, 2006). In contrast, when applying irrelevant masks such perceptual interactions can be excluded as a potential source of the effect. In this case, a different mechanism must be responsible for the reversal of priming effects. The nature of this mechanism remains debated. Lleras and Enns (2006) and Jaśkowski \& Przekoracka-Krawczyk (2005) claimed that some kind of motor inhibition causes inverse priming. This inhibition is triggered by the appearance of a second stimulus following the prime which signals the visual system that the prime induced activity was premature and should therefore be inhibited acting like an "emergency break" to avoid wrong responses (Sumner, 2007). The strength of inhibition depends on the location of the mask and its structure (Jaśkowski, \& Verleger, 2007). Stimuli that contain task-relevant features result in stronger confusion and, thus, these stimuli trigger inhibition of the primed response more effectively (or alternatively they lead to larger inverse priming effects via Object Updating, cf. Krüger, Klapötke, \& Mattler, 2011).

The current demonstration of inverse cue-priming with irrelevant masks cast doubts on the assumption that response alternatives inhibit each other in the generation of inverse priming. Such a mutual inhibition within the motor system does not agree with the reported inverse cue-priming effects with irrelevant masks. In the cue-priming paradigm, neither the prime nor 
cue-stimulus is directly linked to any motor output. With these conditions, the presentation of the mask cannot lead to the inhibition of one response alternative. Instead, if inhibition matters at all, such an inhibitory mechanism must exhibit its influence on prior processing levels: Either the sensory representation of the primed object could be inhibited or the abstract task-set related to it by the instructions. In the current cue-priming task, the solution of the tasks is supposed to be primarily completed in two distinct high-level visual brain areas: the Fusiform Face Area (FFA, Kanwisher, McDurmott, \& Chan, 1997) and the Parahippocampal Place Area (PPA, Epstein, \& Kanwisher, 1998). The mask might trigger inhibition of the primed task-set or its related neural substrate which impedes the execution of that task on congruent trials leading to the observed performance costs. Such modulations of activity in task-specific brain areas by subliminal primes have been successfully demonstrated before in a positive cue-priming paradigm ( $\mathrm{Lau}_{-} \&$ Passingham, 2007). On the other hand, mutual inhibition could take place on a comparatively early level of processing. The processing of the mask could inhibit the perceptual representation of the preceding prime stimulus impeding perceptual processing of a congruent cue more difficult. This early advantage on incongruent trials could translate into actual response tendencies and finally into behavioural outcomes. Current evidence from a psychological refractory period paradigm applied to motor priming pointed to such a perceptual locus of priming effects for relevant metacontrast masks (Krüger, Klapötke, \& Mattler, 2011). Recently, either perceptual habituation (Huber, 2008) or an attentional refractory period (Sohrabi, \& West, 2009) were proposed as a potential mechanism hampering perceptual processing on congruent trial, which thereby explains the inverse priming effect.

Recently, similar to our non-motor cue priming effects, a residual inverse motor priming effect was reported under conditions ruling out perceptual prime-mask interactions and motor inhibition as the source (Klauer, \& Dittrich, 2010; Experiment 4). Perceptual interactions were prevented by presenting the stimulus sequence at very slow pace (prime-mask and masktarget SOA were $180 \mathrm{~ms}$ ) and by using a masking stimulus from a different stimulus family which is comparable to the use of an irrelevant mask. Similar to non-motor priming, primes were not associated with any motor output which was achieved by a trial-to-trial variation of SR-mappings. SR-mappings were signaled to the subject when targets were presented. Nonetheless, Klauer and Dittrich (2010) reported a residual inverse priming effect which they explained in the framework of their evaluation window account. According to this view, primes feed central accumulators representing the abstract response alternatives (which are unrelated from the representation of the response itself) biasing them before mask 
presentation triggers an evaluation monitoring process. Abstract response selection is based on the relative increase of evidence gathered during this evaluation window. Because the primed alternative already reached some non-zero state, increase rates are lower as compared to the unprimed alternative which is still in its initial state, thus facilitating selection of the unprimed, i.e. incongruent response, yielding inverse priming. This is due to diminishing increase rates with growing absolute evidence ('Weber-Fechner-Law', Miller, 1964). The evaluation window account relies on central response selection. Thus, it principally also provides an explanation for non-motor priming effects. Then, the two accumulators would represent the two alternative tasks.

The role of prime visibility in inverse priming

In line with current findings from an inverse target-priming paradigm (Krüger, \& Mattler, in prep.) and several further studies (Eimer, \& Schlaghecken, 2002; Klapp, \& Hinkley, 2002; Klapp, 2005; Sumner, Tsai, Yu, \& Nachev, 2006), we found inverse priming with irrelevant masks to be closely linked to prime visibility. Especially in Experiment 2, inverse effects only occurred within those subjects that proofed to be unable to consciously perceive the prime in the following prime recognition session. In Experiment 3 results were more heterogeneous. While all subjects showed inverse effects in the accuracy domain, a small but reliable RT effect was only found among those subjects showing chance-level performance in the prime recognition part. Strikingly, priming effects were completely independent of visibility when relevant masks were used as also observed before (Krüger, \& Mattler, in prep., Mattler, 2007). This differential influence of prime visibility on priming effects suggests the existence of two completely distinct mechanisms generating inverse priming with both types of masks (Klapp, 2005).

Therefore, at first glance, our findings for irrelevant masks seem to support the motor selfinhibition account (Schlaghecken \& Eimer, 2002; Schlaghecken, Bowman \& Eimer, 2006) which considers perfect masking of the prime as a necessary condition for inverse priming to occur. However, a number of experiments demonstrated inverse priming effects when prime recognition is clearly above chance level (e.g. Klapp, 2005; Lleras \& Enns, 2005; Mattler, 2005, Sumner, Tsai, Yu, \& Nachev, 2006) or even with non-masking distracters leaving prime recognition almost completely unimpaired (Jaśkowski, 2007, 2008; Jaśkowski et al., 2008; Lleras, \& Enns, 2006). Just like masks, such non-masking distracters could either 
contain target-like elements or not. Generally, relevant distracters produced larger inverse priming effects (Jaśkowski, et al., 2008) which could also be accounted for by a broader version of the Object Updating theory neglecting the spatial position of the stimuli in the sequence. In contrast, when irrelevant flankers were used typically positive priming effects were obtained. However, even though primes were perfectly visible irrespective of the temporal position of the mask between the prime and target (prime-target SOA was kept constant) the size of the priming effect was modulated by this temporal parameter. This is difficult to reconcile with the self-inhibition hypothesis which would not ascribe any importance to such a non-masking flanker in the processing of the prime and, therefore, predict equal positive priming effects for all prime-mask SOAs. Even more difficult to explain, Jaśkowski (2008) also achieved inverse priming using an irrelevant flanker by reducing prime duration (to $8 \mathrm{~ms}$ ) in spite of a correct prime identification rate of more than $90 \%$ under these conditions. He interpreted this finding in the framework of his masktriggered inhibition hypothesis assuming that prime visibility is not crucial for inverse priming to occur. Along the same lines, Schlaghecken, Blagrove and Maylor (2008) reported a dissociation of priming effects and visibility in a perceptual learning task. While inverse priming effects remained constant across five sessions, prime recognition improved substantially also questioning a causal relationship of both factors.

However, even though perfect masking does not seem to constitute a necessary prerequisite of inverse priming, considerable evidence for a relation of both factors has been collected (Eimer, \& Schlaghecken, 2002; Klapp, \& Hinkley, 2002; Klapp, 2005; Sumner, Tsai, Yu, \& Nachev, 2006). For example, also Jaśkowski demonstrated that primes followed by a masking stimulus rather than a non-masking flanker generated larger inverse priming effects (Jaśkowski, 2007; Jaśkowski et al., 2008; Jaśkowski, 2008). Therefore, prime visibility seems to matter but not in an all-or-none fashion as initially proposed by Eimer and Schlaghecken. Maybe, an effective mask reduces the initial prime-induced activation making it easier for the inhibitory process triggered by the mask to overcome this initial bias (Jaśkowski, 2008; Sumner, 2007).

Inverse priming with relevant and irrelevant masks: shared or independent mechanisms?

In the three experiments of this study, the mask-cue SOA was varied and a similar timecourse of inverse priming was found compared to previous research (Jaśkowski, 2007, Jaśkowski et al., 2008, Jaśkowski, 2008, 2009; Mattler, 2007; Vorberg \& Lingnau 2005) which even remained the same in the light of changing prime visibility (Experiment 2). 
Inverse priming increased with increasing SOA between the mask and the third stimulus (the target in target-priming and cue in cue-priming). For larger SOAs of about $200 \mathrm{~ms}$ effects declined.

The comparable time-course with relevant and irrelevant masks can be taken as evidence for a common mechanism underlying inverse priming with relevant and irrelevant masks. According to this interpretation, larger inverse priming effects with relevant masks (e.g. Lleras, \& Enns, 2004) might either result from the involvement of a second independent mechanism whose influence is restricted to the use of relevant masks like Object Updating (Co-Active Mechanisms Approach, Krüger, et al., 2011) or because the effectiveness of a common mechanism is modulated by the mask structure (Single Mechanism Approach, Krüger, et al., 2011; cf. Jaśkowski, \& Verleger, 2007). In opposition to the idea of a shared mechanism with both types of masks, however, the effect of prime visibility would then be expected to be comparable between the two masks. But we consistently found an influence of prime visibility only when irrelevant masks were used.

This latter finding could be interpreted as evidence in favour of the view that two independent mechanisms produce inverse priming with both types of masks (Separate Mechanisms Approach, Krüger, et al., 2011). Both mechanisms might possess the same temporal characteristics yielding comparable time-courses of inverse priming effects with both types of masks. For relevant masks, the integration of the stimuli in a common internal representation could depend on the temporal lags between these stimuli in the same fashion as a potential inhibitory mechanism. For the inhibitory mechanism the influence of timing parameters has already been discussed. There is disagreement about the temporal factors being effective in inverse priming. Jaśkowski and colleagues (2008) argued that the prime-mask interval is critical for the size of inverse priming. According to their view, net priming effects represent the aggregation of initial positive prime-induced acitivity bias and inhibition elicited by the mask. Therefore, they proposed that the prime-mask SOA is critical. Short prime mask SOAs might leave less time for the prime to induce activity which is then compensated faster by the presentation of the mask. However, they confounded prime-mask and mask-target SOA allowing for the alternative conclusion that the time window during which inhibition exerts its influence, i.e. the mask-target or mask-cue SOA, determines the size of inverse priming effects as argued by Mattler (2007). Only a sufficiently long delay of cue- or targetpresentation allows for a complete reversal of priming effects. 
Comparing time-courses or the influence of prime visibility among the two types of masks only provides initial clues to the question of whether inverse priming relies on a shared mechanism with relevant and irrelevant masks. Further research is certainly required to answer this question. 


\section{References}

Eimer, M. (1993). Spatial cuing, sensory gating and selective response preparation: an ERPstudy on visuo-spatial orienting. Electroencephalography and clinical Neurophysiology, $88,408-420$.

Eimer, M. (1995). Stimulus-response compatibility and automatic response activation: Evidence from psychophysiological studies. Journal of Experimental Psychology: Human Perception and Performance, 21, 837-854.

Eimer, M. \& Schlaghecken, F. (1998). Effects of masked stimuli on motor activation: Behavioral and electrophysiological evidence. Journal of Experimental Psychology: Human Perception and Performance, 24, 1737-1747.

Eimer, M., \& Schlaghecken, F. (2002). Links between conscious awareness and response inhibition: Evidence from masked priming. Psychonomic Bulletin \& Review, 9, 514-520.

Epstein, R., \& Kanwisher, N. (1998). A cortical representation of the local visual environment. Nature, 392,598-601.

Huber, D.E. (2008). Immediate priming and cognitive aftereffects. Journal of Experimental Psychology: General, 137, 324-347.

Jaśkowski, P. (2007). The effect of nonmasking distractors on the priming of motor responses. Journal of Experimental Psychology: Human Perception and Performance, 33, 456-468.

Jaśkowski, P. (2008). The negative compatibility effect with nonmasking flankers: A case for mask-triggered inhibition hypothesis. Consciousness and Cognition, 17, 765-777.

Jaśkowski, P., Biłuńska, A., Tomanek, M., \& Verleger, R. (2008). Mask- and distractortriggerd inhibitory processes in the priming of motor responses: An EEG study. Psychophysiology, 45, 70-85.

Jaśkowski, P., \& Verleger, R. (2007). What determines the direction of subliminal priming. Advances in Cognitive Psychology, 3 (1-2), 181-192.

Jaśkowski, P., \& Przekoracka-Krawczyk, A. (2005). On the role of mask structure in subliminal priming. Acta Neurobiologiae Experimentalis, 65, 409-417. 
Kanwisher, N., McDermott, J., \& Chun, M.M. (1997). The fusiform face area: A modul in human extrastriate cortex specialized for face perception. The Journal of Neuroscience, $11,4302-4311$.

Kiesel, A., Berner, M.P., \& Kunde, W. (2008). Negative congruency effects: A test of the inhibition account. Consciousness and Cognition, 17, 1-21.

Klauer, K.C., \& Dittrich, K. (2010). From sunshine to double arrows: An evaluation window account of negative compatibility effects. Journal of Experimental Psychology: General, $139,3,490-519$.

Klapp, S. T. (2005). Two versions of the negative compatibility effect: Comment on Lleras and Enns (2004). Journal of Experimental Psychology: General, 134, 431-435.

Klapp, S. T., \& Haas, B. W. (2005). Nonconscious influence of masked stimuli on response selection is limited to concrete stimulus-response associations. Journal of Experimental Psychology: Human Perception and Performance, 31, 93-209.

Klapp, S. T., \& Hinkley, L. B. (2002). The negative compatibility effect: Unconscious inhibition influences reaction time and response selection. Journal of Experimental Psychology: General, 131, 255-269.

Krüger, D., Klapötke, S., \& Mattler, U. (2011). PRP-paradigm provides evidence for a perceptual origin of the negative compatibility effect. Consciousness and Cogntion, 20, 866-881.

Krüger, D., \& Mattler, U. (in prep.). How features oft he mask modulate inverse priming effects of unconscious visual stimuli.

Kuhn, G., \& Kingstone, A. (2009). Look away! Eyes and arrows engage oculomotor responses automatically. Attention, Perception, \& Psychophysics, 71(2), 314-327.

Lau, H.C., \& Passingham, R.E. (2007). Unconscious activation of the cognitive control system in the human prefrontal cortex. The Journal of Neuroscience, 27, 5805-5811.

Lingnau, A., \& Voberg, D. (2005). The time course of response inhibition in masked priming. Perception \& Psychophysics, 67, 545-557.

Lleras, A. \& Enns, J.T. (2004). Negative compatibility or object updating? A cautionary tale of mask-dependent priming. Journal of Experimental Psychology: General, 133, 475-493. 
Lleras, A. \& Enns, J.T. (2005). Updating a cautionary tale of masked priming: Reply to Klapp (2005). Journal of Experimental Psychology: General, 134, 436-440.

Lleras, A., \& Enns, J. T. (2006). How much like a target can a mask be? Geometric, spatial, and temporal similarity in priming: A reply to Schlaghecken and Eimer (2006). Journal of Experimental Psychology: General, 135, 495-500.

Loftus, G. R., \& Masson, M. E. J. (1994). Using confidence intervals in within-subject designs. Psychonomic Bulletin \& Review, 1, 476-490.

Mattler, U. (2003). Priming of mental operations by masked stimuli. Perception and Pschophysics, 65, 167-187.

Mattler, U. (2005). Inhibition and decay of motor and non-motor priming. Perception and Pschophysics, 67, 285-300.

Mattler, U. (2006). On the locus of priming and inverse priming effects. Perception and Psychophysics, 68, 975-991.

Mattler, U. (2007). Inverse target- and cue-priming effects of masked stimuli. Journal of Experimental Psychology: Human Perception and Performance, 33, 83-102.

Pratt, J., Radulescu, P., Guo, R.M., \& Hommel, B. (2010). Visuospatial attention is guided by both the symbolic and the spatial proximity of selected arrows. Journal of Experimental Psychology: Human Perception and Performance, 36 (5), 1321-1324.

Rizzolatti, G., Riggio, L, Dascola, I., \& Umiltà, C. (1987). Reorienting attention across the horizontal and vertical meridians: Evidence in favour of a premotor theory of attention. Neurophysiologica, 25, 31-40.

Schlaghecken F., Blagrove E., \& Maylor, E.A. (2008). No difference between conscious and nonconscious visuomotor control: evidence from perceptual learning in the masked prime task. Consciousness and Cognition. 17(1), 84-93.

Schlaghecken, F., Bowman, H., \& Eimer, M. (2006). Dissociating local and global levels of perceptuo-motor control in masked priming. Journal of Experimental Psychology: Human Perception and Performance, 32, 618-632.

Schlaghecken, F. \& Eimer, M. (2002). Motor activation with and without inhibition: evidence for a threshold mechanism in motor control. Perception and Psychophysics, 64, 148-162. 
Schlaghecken, F., \& Eimer, M. (2006). Active masks and active inhibition: A comment on Lleras and Enns (2004) and on Verleger, Jaśkowski, Aydemir, van der Lubbe, and Groen (2004). Journal of Experimental Psychology. General, 135, 484-494.

Schlaghecken, F., Rowley, L., Sembi, S., Simmons, R., \& Whitcomb, D. (2007). The negative compatibility effect: A case for self-inhibition. Advances in Cognitive Psychology, 3, 227 240.

Sohrabi, A. \& West, R.L. (2009). Positive and negative congruency effects in masked priming: A neuro-computational model based on representation, attention and conflict. Brain Research, 1289, 124-132.

Sumner, P., Tsai, P.C., Yu, K., \& Nachev, P. (2006). Attentional modulation of sensorimotor processes in the absence of perceptual awareness. Proceedings of the National Academy of Sciences USA, 103, 10520-10525.

Sumner, P. (2007). Negative and positive masked priming - implications for motor inhibition. Advances in Cognitive Psychology, 3, 317-326.

Tipples, J. (2008). Orienting to counterpredictive gaze and arrow cues. Perception and Psychophysics, 70 (1), 77-87.

Verleger, R., Görgen, S., \& Jaśkowski, P. (2005). An ERP indicator of processing relevant gestalts in masked priming. Psychophysiology, 42, 677-690.

Verleger, R., Jaśkowski, P., Aydemir, A., van der Lubbe, R.H.J. \& Groen, M. (2004).Qualitative differences between conscious and non-conscious processing? On inverse priming induced by masked arrows. Journal of Experimental Psychology: General, 133, 494-515.

Vorberg, D., Mattler, U., Heinecke, A., Schmidt, T., \& Schwarzbach, J. (2003). Different time courses for visual perception and action priming. Proceedings of the National Academy of Sciences USA, 100, 6275-6280. 
Figure Legends

Figure 1. Stimulus used in Experiment 1 to 3. A relevant mask was used in Experiment 1 whereas Experiment 2 and 3 employed a task-irrelevant masking stimulus. All examples depict incongruent prime-cue relations. Target stimuli were the same across all experiments. One example is depicted.

Figure 2. Results of Experiment 1. (A) Lines represent mean RTs for congruent (filled circles) and incongruent trials (open circles). Error bars indicate standard error of means according to Loftus and Masson (1994). Bars represent mean error rates for congruent (filled) and incongruent trials (white bars). (B) Prime visibility and priming effects. Prime recognition performance (top) and priming effects on RTs (bottom) are depicted separately for those subjects with good and poor prime recognition performance.

Figure 3. Results of Experiment 2. (A) Lines represent mean RTs for congruent (filled circles) and incongruent trials (open circles). Error bars indicate standard error of means according to Loftus and Masson (1994). Bars represent mean error rates for congruent (filled) and incongruent trials (white bars). (B) Prime visibility and priming effects. Prime recognition performance (top) and priming effects on RTs (bottom) are depicted separately for those subjects with best and worst prime recognition performance. The 24 participants were split into three separate groups based on their performance on the prime recognition task. Data from the intermediate group is not depicted.

Figure 4. Results of Experiment 3. (A) Lines represent mean RTs for congruent (filled circles) and incongruent trials (open circles). Error bars indicate standard error of means according to Loftus and Masson (1994). Bars represent mean error rates for congruent (filled) and incongruent trials (white bars). (B) Prime visibility and priming effects. Prime recognition performance (top) and priming effects on RTs (bottom) are depicted separately for those subjects with best and worst prime recognition performance. The 24 participants were split into three separate groups based on their performance on the prime recognition task. Data from the intermediate group is not depicted. 
Figure 1

\begin{tabular}{|c|c|c|c|c|}
\hline & Prime & Mask & Cue & Target \\
\hline \multicolumn{5}{|l|}{ Exp. 1} \\
\hline \multirow{3}{*}{ Exp. 2} & $\ll \ll \ll \ll \ll \ll \ll \ll$ & \multirow{3}{*}{ 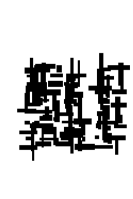 } & 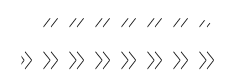 & \\
\hline & $\ll \ll \ll \ll \ll \ll \ll \ll$ & & $\gg\rangle \gg \gg \gg \gg \gg \gg \gg \gg \gg\rangle$ & \\
\hline & $\begin{array}{l}\| \ll \ll \ll \ll \ll \ll \ll \\
" \ll \ll \ll \ll \ll \ll \ll \ll\end{array}$ & & & \\
\hline \multirow{4}{*}{ Exp. 3} & $\hat{v} \hat{v} \hat{v} \hat{v} \hat{v} \hat{v} \hat{v} \hat{v}$ & & $x x_{X} x_{X} X X X X X$ & \\
\hline & $\hat{v} \hat{v} \hat{v} \hat{v} \hat{v} \hat{v} \hat{v} \hat{v}$ & & $X X X X X X X X X$ & \\
\hline & $\hat{v} \hat{v} \hat{v} \hat{v} \hat{v} \hat{v} \hat{v} \hat{v}$ & 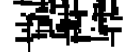 & $X X X X X X X X$ & \\
\hline & $\hat{v} \hat{v} \hat{v} \hat{v} \hat{v} \hat{v} \hat{v} \hat{r}$ & & $\forall X X X X X X X X$ & \\
\hline
\end{tabular}


Figure 2

\section{A}
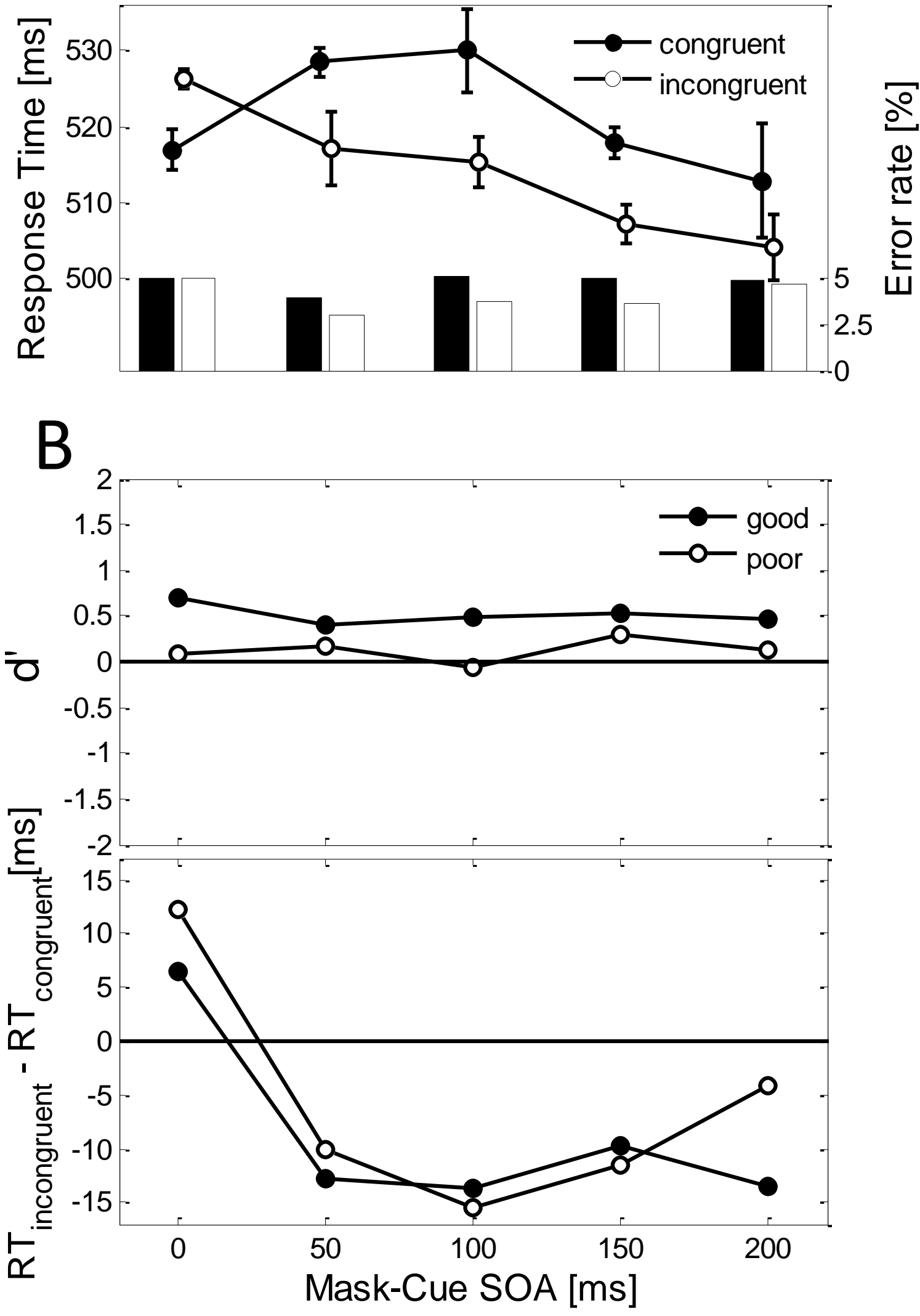
Figure 3

\section{A}
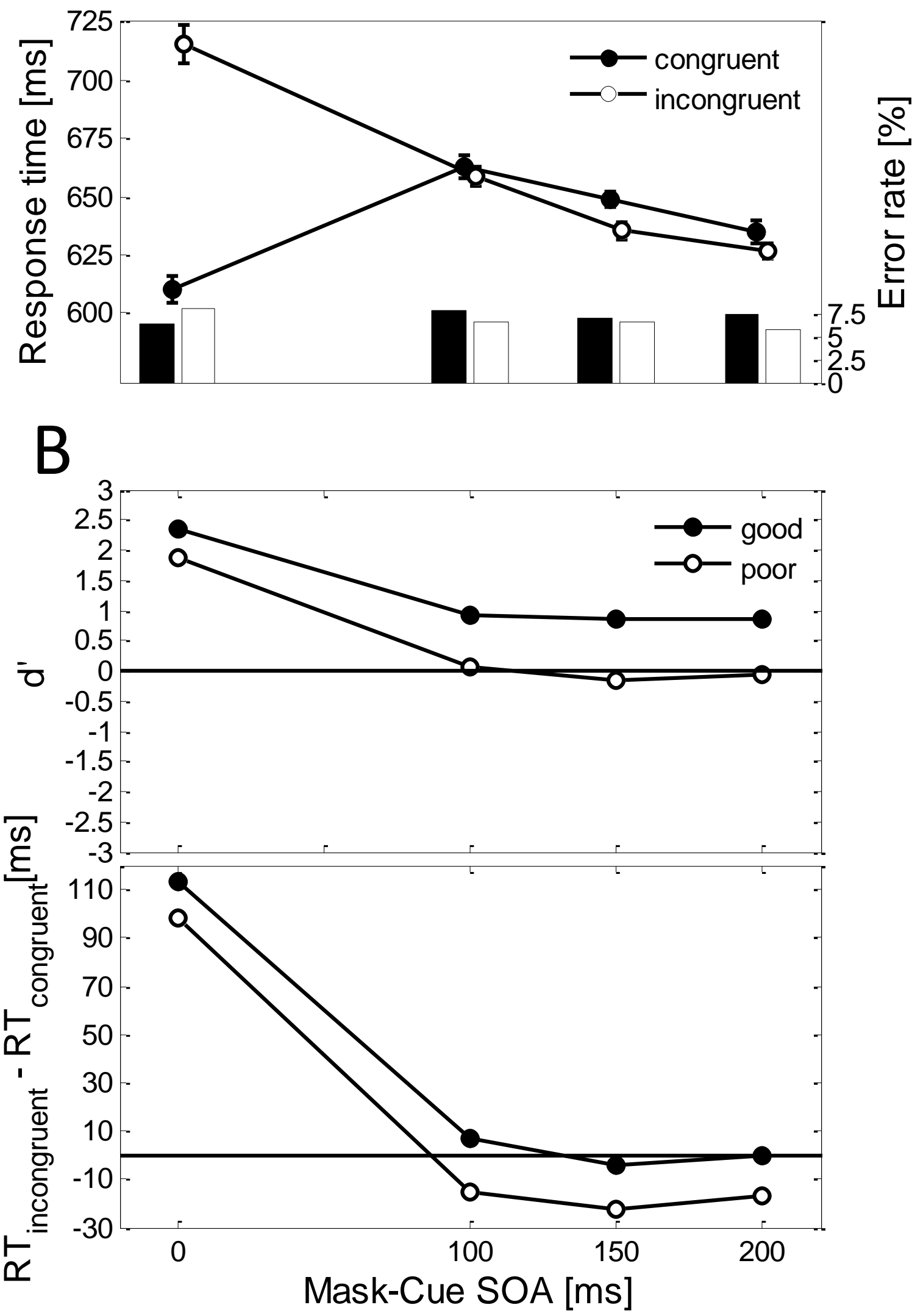
Figure 4

A
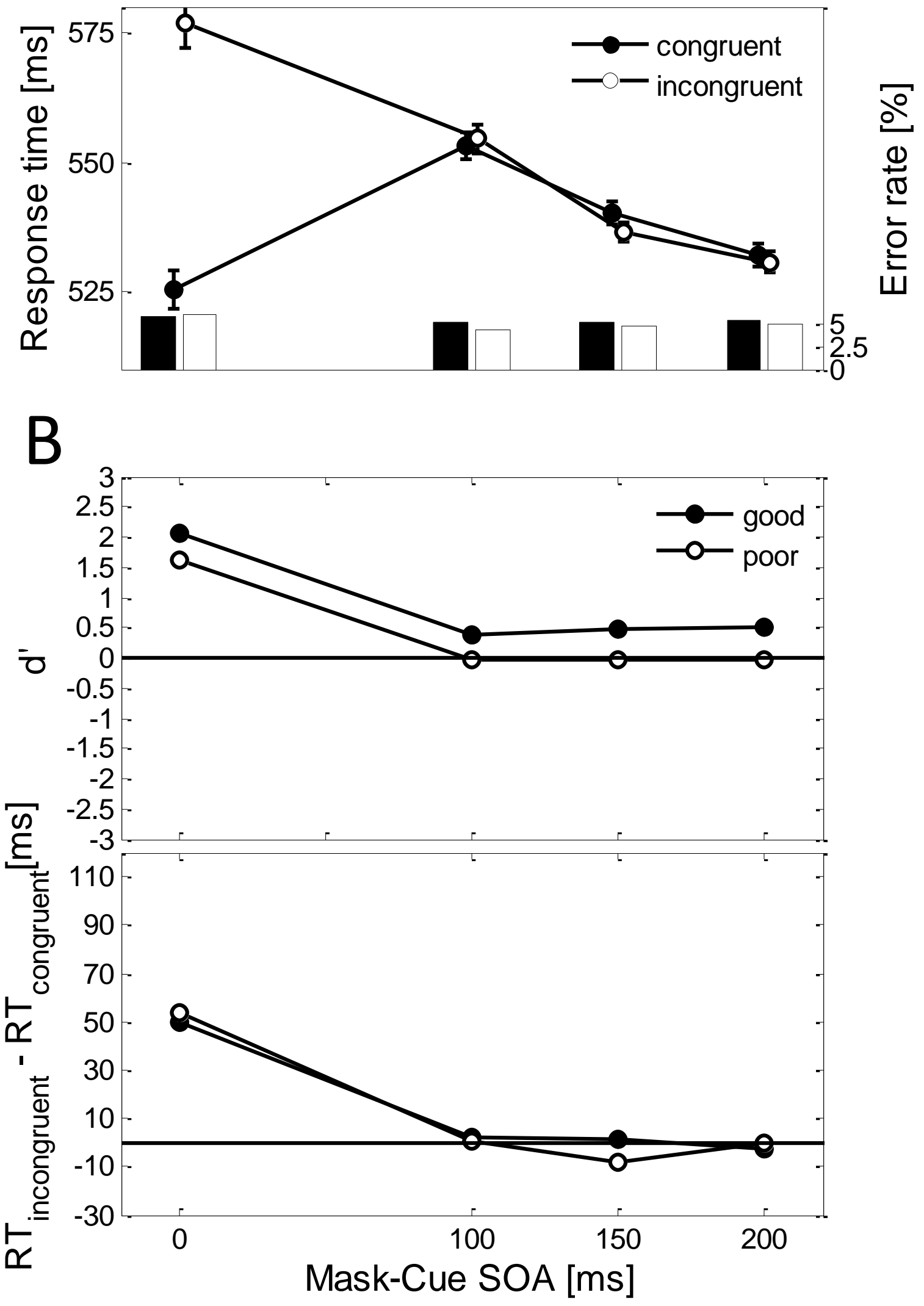


\section{Appendix V}

Krüger, D., Klapötke, S., Bode, S., \& Mattler, U. (submitted). Cognitive control systems in the human cortex susceptible to unconscious stimuli. 


\title{
Cognitive control systems in the human cortex are
}

\section{susceptible to unconscious stimuli}

Abbreviated title: automatic control systems

\author{
Daniel Krüger ${ }^{1}$, Susan Klapötke ${ }^{1}$, Stefan Bode $^{2} \&$ Uwe Mattler $^{1}$ \\ ${ }^{1}$ Georg-Elias-Müller Institute for Psychology, Georg-August University Göttingen, Germany \\ ${ }^{2}$ Psychological Sciences, The University of Melbourne, Australia
}

Journal Section: Behavioral/Systems/Cognitive

Send correspondence to:

Uwe Mattler

Georg-Elias-Müller Institute for Psychology

Georg-August University Göttingen

Gosslerstr. 14

D-37073 Göttingen

Germany

Email: uwe.mattler@psych.uni-goettingen.de

Keywords: priming, negative compatibility effect, consciousness, inhibition

Manuscript includes 6 Figures and 3 Tables

Number of words: 2587 


\begin{abstract}
The inverse priming paradigm can be considered one example which demonstrates the operation of control processes in the absence of conscious experience. We aimed to localize the neural substrates of these control processes using functional magnetic resonance imaging. To this end, prime stimuli were made invisible by two kinds of masks. We used relevant masks which are composed of the superposition of both prime alternatives, and irrelevant masks which are free of task-relevant features. Typically, relevant masks produce larger behavioral inverse priming effects. Results indicate that a network of control units are accessible to unconscious primes, including supplementary motor area (SMA), anterior insula, middle cingulate cortex, and supramarginal gyrus. These brain areas were responsive to invisible primes specifically when relevant masks were use, and only SMA responded also when irrelevant masks were used. Activation in SMA correlated with inverse priming effects of individual participants on reaction time, indicating that these brain areas reflect the size of regulatory demands in the inverse priming paradigm. Findings suggest that various human cognitive control systems in the frontal and parital cortex integrate task relevant stimulus information irrespective of conscious awareness.
\end{abstract}




\section{Introduction}

Cognitive control has been considered one instance which requires conscious processing (e.g., Norman and Shallice, 1986). However, several behavioral studies have shown that stimulus processing is influenced by a preceding stimulus, even if the first stimulus is not consciously perceived (e.g. Mattler, 2003; Vorberg, et al., 2003). Typically, performance benefits are observed when prime and target belong to the same category (congruent condition) as opposed to belonging to alternative categories (incongruent condition). On this background, it is a matter of debate whether unconscious stimuli can also modulate cognitive control operations (e.g., Rees, et al., 2002; Dehaene, et al., 2003). Here we aimed to examine the susceptibility of control operations to unconscious stimuli with a priming paradigm which enables the variation of the demands for control operations to perform the task. To this end we employed the inverse priming paradigm which produces a reversal of priming effects by presenting a masking stimulus after the prime which is separated by the following target stimulus. Inverse priming effects (which are also known as "negative compatibility effects") are characterized by better performance on incongruent trials (e.g., Eimer and Schlaghecken, 1998). This reversal of priming effects has been related to the operation of control processes which resolve the conflict that results from inconsistent response activations (Boy, Husain, et al., 2010; Boy, Evans, et al., 2010). According to this view, the inverse priming effect demonstrates the neural correlates of cognitive control mechanisms which operate automatically outside of consciousness. The size of inverse priming depends on the stimulus onset asynchrony (SOA) between mask and target stimulus (Mattler, 2007; Schlaghecken, and Eimer, 2000) and on the structure of the mask (e.g. Lleras, and Enns, 2004). With short mask-target SOAs priming effects tend to be positive, with increasing mask-target SOA effects inverse priming effects occur. As outlined below, we assume that this reversal of priming effects with long SOA is linked with increased control processing on congruent trials. With masks that consist of task-relevant features (e.g. by simply superimposing both prime alternatives) inverse priming effects are typically larger than with masks that consist of task-irrelevant features. Our study was designed to differentiate the automatic control processes which govern inverse priming effects with relevant and irrelevant masks.

The Object Updating account of inverse priming (Lleras and Enns, 2004) provides an explanation for the modulation of inverse priming effects by the mask structure. It assumes that due to a novelty bias in visual processing all mask elements that are distinct from the 
prime become more salient. Therefore, the processing of prime incongruent features of taskrelevant masks is enhanced which in turn leads to facilitated processing of prime-incongruent targets. However, Object Updating is not sufficient to explain inverse priming with taskirrelevant masks (e.g. Schlaghecken, and Eimer, 2006). We have distinguished three approaches to this issue in the literature (Krüger, Klapötke and Mattler, 2011). According to the Co-active Mechanisms approach, an inhibitory mechanism is effective with both types of masks and Object Updating contributes to the effect only with relevant masks (Lleras, and Enns, 2006; Schlaghecken et al., 2007). According to the Single-Mechanism approach, the inhibitory mechanism generates inverse priming effects with both kinds of masks but for some reason it is more productive with relevant masks (Jaśkowski and Verleger, 2007). According to the Separate Mechanisms approach, Object Updating accounts for the entire effect with relevant masks, and an inhibitory mechanism is only operating with irrelevant masks (Klapp, 2005).

Here, we applied functional magnetic resonance imaging (fMRI) to localize cognitive control operations involved in inverse priming with relevant and irrelevant masks. To this end, we varied the structure of the mask between trials. Previous fMRI studies employed only irrelevant masks and results suggest a crucial role for the supplementary motor area in inverse priming (Boy, Evans, et al., 2010; Boy, Husain et al., 2010; Sumner, et al., 2007). Our approach enables an assessment of the generality of this finding for relevant masks. The Coactive mechanisms and Single-Mechanisms approaches predict that the same brain regions are involved in inverse priming with relevant and irrelevant masks. The Separate-Mechanisms approach predicts distinct brain regions in the case of relevant and irrelevant masks.

\section{Materials and Methods}

Participants. 28 healthy students from the University of Goettingen were recruited to participate in the four sessions of the experiment. Two of them voluntarily quitted during the first two practice sessions, two subjects did not perform the task correctly, one showed anatomical irregularities, and another subject's data were lost due to technical failure. The remaining 22 subjects constituted the final sample ( 7 male, mean age 22.6 years, ran ging from 19 to 27 years). All had normal or corrected-to-normal vision and were right-handed according to self-report. Before participation, subjects gave written informed consent and completed a questionnaire to ensure MRT safety requirements and to rule out a history of neurological or psychiatric illness. Participants received an allowance of $51 €$ for their 
participation. The study was approved by the Ethics Committee of the Medical Faculty of the University of Goettingen.

Stimuli. The trial structure and timing parameters were the same throughout all four sessions of the experiment (see Figure 1). Prime stimuli were left- or rightward pointing double arrows $(<<$ or $>>)$ presented at the center of the screen. Each target stimulus consisted of a pair of identical double arrows, pointing towards the left or to the right, presented below and above fixation. Targets pointed to the same side as the primes on half of the trials (congruent condition) and to opposite sides otherwise (incongruent condition). Primes were followed by a masking stimulus presented at the center of the screen. A relevant mask was presented on half of the trials and an irrelevant mask otherwise. The relevant mask consisted of a superposition of both prime alternatives. The irrelevant mask consisted of 130 lines of different length and width each approximately centered on the 13 x 10 intersections of a virtual grid with a small random spatial jitter. A new irrelevant mask was generated for each trial. All stimuli were presented in black on a white background. Stimulus presentation was realized with a CRTmonitor during the first two practice sessions in the lab, and with MRT-compatible LCDglasses during the last two sessions in the scanner, both running at $60 \mathrm{~Hz}$. All double arrows subtended $1.5^{\circ} \mathrm{x} 1^{\circ}$ of visual angle. The two double arrows which constituted the target stimuli were presented $2.3^{\circ}$ above and below fixation, respectively. The virtual grid underlying the structure of the irrelevant masks subtended $1.7^{\circ} \mathrm{x} 1.1^{\circ}$ of visual angle. The color of the fixation point was changed for $1000 \mathrm{~ms}$ to give feedback at the end of each trial (green and red following correct and incorrect responses, respectively).

Tasks. (a) Choice-reaction time task: During the initial two practice sessions and the third session (in the scanner), subjects were required to judge the orientation of the target stimuli as fast as possible avoiding errors. When the target arrows pointed to the left a response with the left index finger was required and otherwise a response with the right index finger. (b) Prime recognition task: To estimate the visibility of primes on the LCD-glasses of the scanner, we conducted a final prime recognition session with the same setup as in the previous session in the scanner. In this session, subjects were informed about the presence of the prime and they had to report its orientation in the same way as during the first three sessions but without speed stress. Again, a leftward pointing prime required a response with the left index finger and a rightward pointing prime with the right index finger. Trial wise error feedback was given on each task. 
Procedure. Prior to the MRT-session participants performed two identical practice sessions outside the scanner. The temporal structure of the trials was the same in all four sessions of the experiment (Figure 1). The beginning of each trial was signaled by a large fixation cross presented for $500 \mathrm{~ms}$. The prime followed $300 \mathrm{~ms}$ after the offset of the fixation cross for 17 ms. The mask followed $17 \mathrm{~ms}$ after prime offset for $100 \mathrm{~ms}$. The mask-target SOA varied randomly between trials with values of 33 or $150 \mathrm{~ms}$. Targets were presented for $100 \mathrm{~ms}$. After target onset, the computer waited $1433 \mathrm{~ms}$ or $1317 \mathrm{~ms}$ for a response on trials with short or long SOA, respectively. Within this sequence of stimuli a fixation dot was presented when no other stimulus was presented. The intertrial interval was $1033 \mathrm{~ms}$. Thus, in total the duration of each trial was $4333 \mathrm{~ms}$. In the MRT-session, volume acquisition was triggered with a temporal jitter relative to the trial onset because the repetition time of the scanner (TR) was 2 secs. Therefore, the jitter varied between 0, 333, 667, 1000, 1333, and $1667 \mathrm{~ms}$. Each experimental condition was realized equally often with each of these jitter conditions.

Design. In each session subjects performed eight runs with 96 trials each. Only the data of the two final sessions in the scanner were analyzed. We used a $2 \times 2 \times 2 \times 2$ repeated measures design with factors Prime Orientation (left vs. right), Target Orientation (left vs. right), SOA (33 vs. $150 \mathrm{~ms}$ ), and Mask Structure (relevant vs. irrelevant). Each of these 16 conditions occurred six times per run and was combined once per run with each possible jitter between trial onset and the onset of volume acquisition. Thus, we acquired 48 trials in each experimental condition. Apart from the instructed task, choice-RT and prime recognition sessions were identical. Response time (RT), error rate, and the BOLD signal served as dependent measures in the choice-RT session. The analysis of prime recognition performance focused on the effects of independent variables SOA and Mask Structure on the dependent measure percentage of correctly reported prime stimuli.

Data acquisition. Participants were scanned with a 3.0 T MRI System (Siemens Magnetom Trio). An echo-plannar imaging (EPI) sequence was implemented. During the choice-RT task in the scanner we acquired 33 slices aligned to the AC-PC plane in ascending order (TR = $2000 \mathrm{~ms}, \mathrm{TE}=30 \mathrm{~ms}$, flip angle $=70^{\circ}$, matrix 64 × 64, spatial resolution: $3 \times 3 \times 3.6 \mathrm{~mm}$ with $0.6 \mathrm{~mm}$ gap). For each run, 215 EPI T2*-weighted whole brain images were acquired. The first two volumes of each run were discarded to allow for T1 saturation effects. Preceding functional data acquisition, a high-resolution T1-weighted anatomical image was acquired using a MPRage sequence (spatial resolution $1 \times 1 \times 1 \mathrm{~mm}$ with no gap, TE $=3.26 \mathrm{~ms}$, TR = $2250 \mathrm{~ms}$, flig angle $=9^{\circ}$. 
Data analyses. MRT-data were analyzed using SPM8 (http://fil.ion.ucl.ac.uk/spm; Friston et al., 1994). First, all functional and the anatomical images were reoriented such that the commissura anterior became the origin of the image. Second, ArtRepair 4 (Mazaika, et al., 2009) was used to remove bad slices and to eliminate extracerebral noise. Bad slices were replaced by an interpolation of the preceding and following scan. On average $1.0 \%$ of all slices were corrected by this procedure (ranging from 0.0 to $4.1 \%$ between subjects). Next, a slice time correction compensated for varying slice acquisition times. All functional images were realigned to the first image of the first run to correct for head movement. At the same time, a mean image of all functional images was generated. Then, the anatomical image was co-registered to this mean image and segmented into gray and white matter and cortico-spinal fluid proportions. Parameters for the normalization to the template image provided by the Montreal Neurological Institute (MNI template) were obtained from the segmented anatomical image and applied to all functional and the anatomical images. The normalized functional images were smoothed using a Gaussian Kernel (full width at half maximum: 6 $\mathrm{mm})$. Low frequency artifacts were removed by applying a high-pass filter to the time-series data at each voxel with a cutoff at $128 \mathrm{~s}$.

Single subject analyses were performed by fitting a general linear model (GLM) to the time-series data. The 16 experimental conditions were used as explanatory variables. Separately for every run, each condition was modeled as a time-series of events based on prime onsets, excluding error trials. The resulting stick function was convolved with the canonical haemodynamic response function as implemented in SPM 8. Additionally, seven covariates-of-no-interest were added to the GLM: one regressor modeled error trials and six regressors modeled head movements ( 3 translation, 3 rotation parameters given by the realignment algorithm). Effects of our experimental manipulation on the BOLD signal were assessed by linear combinations of the GLM parameter estimates (beta values). At the level of group statistics, the reliability of effects was tested by one-sample t-tests.

Statistical Analysis of fMRI data. Data analyses are based on a response activation model of inverse priming which is described below. The model predicts different effects of SOA on congruent and incongruent trials in distinct brain areas. To test the assumptions of the model the main effects of SOA on congruent trials is compared to the main effect of SOA on incongruent trials for each mask separately. Additionally, the model enables the analysis of brain areas that are related to response conflict and control operations. To distinguish control processes that are related to relevant masks from those related to irrelevant masks and control 
processes which are involved with either type of mask, we determined brain areas which show specific patterns of activation indicative for control processes (i) exclusively on trials with relevant masks, (ii) exclusively on trials with irrelevant masks, and (iii) for both masks.

Our analysis of control processes is derived from a simple response activation model of inverse priming. Consistent with electrophysiological measures of response activation in the inverse priming paradigm, the model assumes that the processing of prime, mask, and target induces a sequence of response activations and control processes which serve to compensate premature response tendencies. The sequence of processes depends on Congruency and SOA (see Figure 2A). According to the model, increased regulatory demands are predicted only on congruent trials with long rather than short SOA (Con: $150 \mathrm{~ms}$ SOA > $33 \mathrm{~ms} \mathrm{SOA})$. However, this contrast is confounded with the effects of the differences in the physical stimulus sequences with long and short SOA. To control for these differences in visual processing, the effect of long SOA was compared to short SOA on incongruent trials (Inc: $150 \mathrm{~ms} \mathrm{SOA}>33 \mathrm{~ms}$ SOA). This is based on the assumption that regulatory demands do not vary with SOA on incongruent trials where the mask-induced reversal of response activation drives accumulation towards the correct response and target induced activation simply continues this tendency (see Figure 2A). Three predictions can be derived from this model. First, the main effect of SOA on incongruent trials should be restricted to visual brain areas. Second, on congruent trials all these areas should exhibit a comparable main effect of SOA. Third, brain areas which are responsive to SOA variation on congruent but not on incongruent trials can be considered neural correlates of cognitive control operations.

To test these predictions, we examined the SOA effect on congruent and incongruent trials in the entire brain and compared the main SOA effect on congruent trials to that on incongruent trials. We aimed to distinguish brain regions according to five specific patterns of activation (Figure 2B). First, to identify brain areas which are exclusively responsive to physical differences in the stimulus sequence but not to varying control demands we searched for voxels which respond to SOA on congruent and incongruent trials in a conjunction analysis (see Figure 2B, left panel). Conjunction analyses determine brain regions that are responsive to two (or more) experimental manipulations at the same time. From these voxels we excluded voxels which showed a larger SOA effect on congruent trials compared to the SOA effect on incongruent trials.

Second, to identify brain areas which are related to both, visual processing and control processes we searched for voxels which respond with a larger SOA effect on congruent than 
on incongruent trials (Figure 2B, middle panel). This was accomplished by a conjunction analysis of SOA effects on congruent trials, SOA effects on incongruent trials, and the interaction of SOA and Congruency specified by larger SOA effects on congruent trials.

Third, to identify brain areas which are specifically related to control operations but not to perceptual operations we searched for voxels which respond to SOA on congruent trials but not on incongruent trials (Figure 2B, right panel). This was accomplished by a conjunction analysis of SOA effects on congruent trials and the interaction of SOA and Congruency, and we excluded voxels that showed an SOA effect on incongruent trials. This conjunction analysis was tailored in three ways: To identify brain areas which are exclusively related to control operations on trials with relevant (irrelevant) masks, we excluded voxels which showed an SOA effect on incongruent trials and the interaction SOA x Congruency with irrelevant (relevant) masks. To identify brain areas which are related to control operations with either mask, the conjunction analysis had to be significant with either mask.

Finally, we examined the relation between the activation in the supplementary motor area (SMA) which is related to control operations and the inverse priming effect on performance measures of RT. This analysis was stimulated by a recent finding of Boy and others (2010) which demonstrated a relationship between the individual GABA-level within the SMA and the size of the behavioral inverse priming effect. To this end, we correlated individuals' inverse priming effect on RTs - determined as the difference of RT on incongruent minus RT on congruent trials - with individuals' increase in SMA activity on congruent trials from short to long SOA. This analysis was performed for each mask separately, first, with the SMA voxel that showed the largest SOA effect on congruent trials, and second, with all SMA voxels which responded to SOA on congruent trials. 


\section{Results}

\section{Behavioral results}

$R T$ data. Priming effects depended on SOA and Mask Structure as indexed by the three-way interaction of Congruency, SOA, and Mask Structure in a repeated measures ANOVA, $\mathrm{F}(1,21)=57.7, \mathrm{p}<.0001$ (see Figure 3). With short SOAs, significant positive priming effects were observed $(\mathrm{t}(21)=12.4$, and 13.2, $\mathrm{p}<.0001$ for relevant and irrelevant masks, respectively) with faster responses on congruent trials (relevant mask: $322 \mathrm{~ms}$, irrelevant mask: $320 \mathrm{~ms}$ ) as compared to incongruent trials (365 ms and $363 \mathrm{~ms}$, respectively). These effects were independent of mask structure because the interaction of Congruency and Mask Structure was not significant $(\mathrm{F}(1,21)<1)$. With long SOAs, however, inverse priming effects were obtained: responses were slower on congruent trials (350 ms and $332 \mathrm{~ms}$ ) when compared to incongruent trials (322 and $330 \mathrm{~ms}$ ). The size of inverse priming effects differed between the two masks as reflected in the significant interaction of Congruency and Mask Structure, $\mathrm{F}(1,21)=60.1, \mathrm{p}<.0001$. With the relevant mask, a substantial inverse priming effect of $-28 \mathrm{~ms}$ was found which significantly differed from zero, $\mathrm{t}(21)=-6.8, \mathrm{p}<.0001$ (one-tailed). In contrast, with the irrelevant mask, however, the inverse priming effect did not reach significance $(-2 \mathrm{~ms}, \mathrm{t}(21)=-.5, \mathrm{p}=.33$, one-tailed $)$.

Accuracy Data. Errors occurred on $2.9 \%$ of all trials. Accuracy data were arc-sine transformed and subjected to a repeated measures ANOVA. Priming effects were significantly modulated by SOA and Mask Structure, $F(1,21)=8.3, p<.0001$ (see Figure 3). With short SOA, significant positive priming effects occurred with fewer errors on congruent (relevant mask: $\mathrm{M}=0.4 \%$, irrelevant mask: $0.5 \%)$ as compared to incongruent trials $(2.7 \%$ and $3.4 \%$, respectively; $\mathrm{t}(21)=4.1$ and $3.4, \mathrm{p}<.01$, for relevant and irrelevant masks, respectively. The interaction between Congruency and Mask Structure was not significant, $\mathrm{F}(1,21)<1$. With long SOAs, inverse priming effects occurred with more errors on congruent (5.8\% and 5.8\% for relevant and irrelevant masks, respectively) as compared to incongruent trials $(1.0 \%$ and $3.9 \% ; \mathrm{t}(21)=-.4 .2, \mathrm{p}<.001$, and $-2.0, \mathrm{p}<.03$, one-tailed, for relevant and irrelevant masks, respectively). Inverse priming effects were larger with relevant masks ($4.8 \%)$ than with irrelevant masks $(-1.9 \%)$ as reflected in the significant interaction of Congruency and Mask Structure $(\mathrm{F}(1,21)=7.3, \mathrm{p}<.02)$, resembling the effects on RT.

Prime Recognition Performance. Overall, participants correctly reported the orientation of the prime stimulus on $50.7 \%$ of the trials. The effect of Mask Structure was significant $(\mathrm{F}(1,21)$ 
$=9.8, \mathrm{p}<.01)$ because the frequency of correct responses was smaller with relevant masks (49.0\%) than with irrelevant masks $(52.3 \%)$. However, prime recognition performance did not differ significantly from chance level neither with relevant $(\mathrm{t}(21)=-1.1, \mathrm{p}>.28)$ nor with irrelevant masks $(t(21)=1.5, p>.14)$. No other effect reached significance.

\section{fMRI results}

Perceptual effects of SOA. For each type of mask we examined main effects of SOA separately on congruent and incongruent trials. On incongruent trials, increased brain activity with long as compared to short SOA was restricted to the visual system (Figure 4A). For both types of masks we found symmetrical clusters of activation at the transition from occipital to temporal lobe with the maxima located on the left middle occipital gyrus and right inferior temporal gyrus. With irrelevant masks, SOA modulated the activity of larger brain regions than with relevant masks (see Table 1). This finding is consistent with our assumption that cognitive control demands do not vary with SOA on incongruent trials (see Figure 2A).

On congruent trials, the effect of SOA involved the same brain regions as on incongruent trials, but a large number of additional regions were also significantly more activated with long rather than short SOA (Figure 4B). These additional brain areas included the SMA, precentral gyrus and the postcentral gyrus (for a complete summary see Table 2). This finding is consistent with our assumption that the control demands increase on congruent trials when SOA increases (Figure 2A).

To separate purely perceptual effects from control related SOA effects we determined voxels with comparable SOA effects on congruent and incongruent trials, and excluded voxels with different SOA effects in these two conditions (left panel of Figure 2B). Purely perceptual effects of SOA were found bilaterally in virtually the same higher visual areas with relevant and irrelevant masks in the anterior occipital lobe at the transition to the temporal lobe (Figure 4C). The focus of perceptual SOA effects for each mask was located within a cytoarchitectonically defined region called hoc5 corresponding to the area V5/MT+ (Malikovich et al., 2007).

Perceptual and Control related Effects of SOA. To identify brain regions which are involved in both, perceptual and control related operations, we determined those voxels in which the SOA effect on congruent trials exceeded the SOA effect on incongruent trials (middle panel in Figure 2B). However, with the preset statistical threshold we did not find any brain regions with this pattern of activity with neither type of mask. 
Control Related Effects of SOA: First, we identified brain regions that are involved in control operations with either mask. Then, we identified brain regions that are involved exclusively with relevant or irrelevant masks. Increased activity with long SOA as compared to short SOA only on congruent trials and no SOA effect on incongruent trials (Figure 2B, right panel) was found for both types of masks only in one cluster within the SMA (Figure 5; Table 3). According to our model, the activation of this brain area is related to control processes which are relevant for inverse priming effects with either type of mask. Therefore, this brain area could represent a basic mechanism which is operating with both, relevant and irrelevant masks.

Next, we tried to identify control related activation which is exclusively found with one type of mask. With this tailored analysis we did not find any brain region which was activated only on trials with irrelevant masks. In contrast, nine clusters were exclusively found with relevant masks (Figure 5; Table 3). On the one hand, mask specific control related activation was found in an additional part of the SMA. On the other hand, additional clusters were situated ventral to the SMA within the posterior part of the anterior cingulate cortex, the rostral cingulate zone (RCZ, Picard, and Strick, 1996). The same specific pattern of activation was also found within insula and the surrounding rolandic operculum bilateral but more pronounced within the right hemisphere, and also within the left postcentral gyrus and the left supramarginal gyrus with peak activations in the cytoarchitectonically defined subregions PFcm and PFop (Caspers, et al., 2006). Beyond this, the same pattern of activation was also found in regions of the right cerebellum.

Correlation of SMA activity and behavioral inverse priming effects. We performed correlation analysis in order to investigate whether control related SOA effects in the SMA were related to behavioral priming effects. First, we determined the voxel, which was most strongly affected by SOA on congruent trials (identified by the largest t-value) for the conjunction of both masks in the entire group of subjects $(0,-4,54 \mathrm{MNI}$, located in the SMA). Then we calculated the correlation between the neural control related effect ( $\beta$ congruent SOA=150 $-\beta_{\text {congruent }}$ $\mathrm{SOA}=33)$ in this voxel and the behavioral inverse priming effect $\left(\mathrm{RT}_{\text {incongruent }}-\mathrm{RT}_{\text {congruent }}\right)$ on trials with long SOA. Significant negative correlations were observed for both, the relevant mask $(\mathrm{r}=-.43, \mathrm{p}<.05)$ and the irrelevant mask $(\mathrm{r}=-.5, \mathrm{p}<.02$; Figure 6A). These correlations indicate that subjects with a larger increase of SMA activation on congruent trials with increasing SOA showed larger behavioral inverse priming effects. Second, to rule out that our finding was restricted to one voxel only, we determined correlations for all SMA 
voxels as indicated by the SMA-ROI from the MARSBAR toolbox for SPM (http://marsbar.sourceforge.net/, Tzourio-Mazoyer et al., 2002). For this, we selected all voxels within the SMA whose activity was larger at long as compared to short SOA in the group analysis at $\mathrm{p}<.001$ (yielding 305 voxels). An analysis of the correlations revealed that 159 SMA-voxels showed a significant $(\mathrm{p}<.05)$ negative correlation with relevant masks, and 40 voxels with irrelevant masks. The entire distributions of correlation coefficients for all selected SMA voxels are given in Figure 6B.

\section{Discussion}

The present study was designed to shed light on the neural correlates of cognitive control processes that are involved in inverse priming. To this end, trials with low cognitive control requirements were compared to trials with high control demands. In addition, we used relevant and irrelevant masks to render primes invisible. Rigorous psychophysical testing revealed that prime recognition performance was at chance level with either type of mask. This finding suggests that participants have not consciously perceived the effective stimuli in our study. Nonetheless, the activation of several brain areas which are related to high level control processes has been modulated by unconscious primes. Findings suggest that the SMA is the common neural site of control processes in inverse priming on trials with relevant and irrelevant masks. Exclusively on trials with relevant masks, the activation of several other brain areas in the frontal and parietal cortex was modulated by unconscious primes which initiated cognitive control operations. These findings support the view that inverse priming is governed by a common mechanism which operates with either type of mask, and an additional mechanism which operates only on trials with relevant masks and generates the enhanced inverse priming effect in behavioral measures. In other words, the present results support the Co-active Mechanisms approach (Krüger, et al., 2010). Most important, however, our findings demonstrate that various control operations in frontal and parietal cortex are susceptible to unconscious stimuli.

The present approach examined cognitive control processes in inverse priming by comparing brain activity on congruent trials with long and short mask-target SOAs. According to our response activation model, regulatory demands increase on congruent trials with increasing SOA because the duration of the mask-induced activation of the unprimed response alternative increases and the amount of control operations to overcome this 
misleading response tendency increases too. As mentioned above, however, the contrast between brain activation with short and long SOAs is confounded by the effect of the differences in the temporal stimulus sequence. To control for these perceptual sequence effects, we analyzed SOA effects on incongruent trials. SOA effects on incongruent trials are a measure of all effects which are not contaminated by changes in regulatory demands because incongruent trials the target simply confirms the mask-induced reversal of response activation irrespective of SOA. These assumptions were nicely confirmed by our findings. Irrespective of Mask structure and Congruency, the different stimulus sequences modulated activity within area V5/MT+ (Figure 5C; Malikovich et al., 2007) which has been associated with motion perception (Willms, et al. 2005). Interestingly, Goebel and Colleagues (1998) reported that apparent motion evoked larger activity in this brain region than a flicker control condition. We suggest that the sequence of central prime and mask presentation followed by the peripheral presentation of target stimuli has induced motion signals in our participants. Perception of apparent motion critically depends on the spatial separation and the temporal delay between the stimuli (Shepard, and Zare, 1983). Therefore, the motion signals generated by our stimulus sequence might have been stronger with long rather than short SOA.

SMA has been related to inverse priming with irrelevant masks in previous studies. A patient with a focal lesion in the SMA showed positive priming in contrast to another patient with a lesion in medial frontal cortex sparing the SMA who showed normal inverse priming effects on manual responses (Sumner, et al., 2007). Previous fMRI data showed that SMA activation on congruent trials was larger than on incongruent trials at a mask-target SOA of $150 \mathrm{~ms}$ (Boy, Husain, et al. 2010). Our data extend these findings by demonstrating a general role of the SMA in inverse priming with both, relevant and irrelevant masks. For both types of masks, activity in the SMA seems to mirror the need for control processes to inhibit outdated response tendencies. A comparable relation between SMA activity and response conflict has been found with the Eriksen Flanker task (Hazeltine, et al., 2000). This view is consistent with previous research that suggests a relation between SMA and motor control processes, which are involved in the suppression of planned but currently inappropriate response tendencies (Swick, et al., 2011), the sudden adaptations of planned movements (Matsuzaka, and Tanji, 1996), response inhibition in reaction to sudden task changes (Chen, et al., 2010), and the instantiation of currently relevant movements (see Nachev et al., 2008). Moreover, a neurochemical study found larger inverse priming effects in individuals with lower concentrations of the inhibitory neurotransmitter GABA within the SMA (Boy, Evans, et al., 
2010). Thus, SMA is one instance of a motor control system in the human frontal cortex which is susceptible to unconscious stimuli.

With relevant masks we found additional brain areas where the metabolic activation increased when the need for control processes was increased by unconscious stimuli. These additional operations might be responsible for the larger inverse priming effects on behavioral measures with relevant as compared to irrelevant masks. One cluster which was specifically involved with relevant masks is situated in the middle frontal cortex, in the RCZ which has previously been associated with response monitoring, response conflict and decision uncertainty (Roger et al., 2010, Ridderinkhof, van den Wildenberg, et al., 2004; Ridderinkhof, Ullsperger, et al., 2004). Activation in bilateral clusters within the insula lobe were also correlated with regulatory demands: in the right-hemisphere, the anterior part of the insula and a cluster in the posterior portion showed the specific control related pattern of activation. Within the left hemisphere, a single cluster was found in the posterior region of the insula. The meta-analysis of Swick and colleagues (2011) identified the anterior insula as a common module for the successful performance for withholding a prepared response and also reported a preponderance of the right hemisphere which accords with the present data. MCG and insula are central components of the core task-set system according to meta-analyses of Dosenbach and colleagues $(2006 ; 2007)$ which controls goal-directed behavior through the stable maintenance of task sets. Thus, our data suggest that unconscious stimuli have access to this cingulo-opercular task-set network. This conclusion is in line with a recent study of Van Gaal, Ridderinkhof and colleagues (2010) who report an effect of unconscious no-go stimuli on preSMA and bilateral inferior frontal regions. Activity in the left post-central gyrus and in the left supramarginal gyrus (SMG) within the inferior part of the parietal lobe was also modulated by unconscious primes. Rushworth (2001) proposed a manual motor attention system in the left supramarginal gyrus and intraparietal sulcus which is confirmed by a recent MRI study (Cotti, et al., 2011). Hypothetically, increased response conflict at longer SOAs might be accompanied by corresponding preparatory processes with respect to motor attention for the upcoming movement even though the effective stimuli which cause the response conflict remain unconscious. Together, these areas seem to constitute a second mechanism which contributes to inverse priming in the case of relevant masks.

Although the main focus of the present study was an examination of the susceptibility of control operations to unconscious stimuli, the present findings contribute also to the understanding of inverse priming effects. The anatomical location of the control areas that are 
modulated by the congruency between unconscious primes and visible target stimuli which we reported above, seems to be consistent with the view that inverse priming is generated in the response system (Boy and Sumner, 2010; Jaśkowski and Verleger, 2007; Klapp and Hinkley, 2002; Lleras, and Enns, 2006; Schlaghecken and Eimer, 2002) rather than at perceptual levels of processing (Huber, 2008; Krüger, et al., 2011; Mattler, 2006; 2007; Sohrabi and West, 2009). Note, however, that this interpretation depends on the current functional specification of the responsive brain areas. Nonetheless, a comparison of the literature suggests that different amounts of response compatibility of the primes could explain why some studies report evidence for a perceptual origin of inverse priming and other studies evidence in favor of a post-perceptual mechanism (cf. Jaśkowski, \& Ślósarek, 2007). Evidence for a post-perceptual origin is provided mostly by studies which employed arrow stimuli. Arrows are characterized by a high degree of dimensional overlap to the response set (Kornblum, et al., 1990) which could result in automatic activation of the corresponding response (D’Ostilio, \& Garraux, 2011; Georgopoulos, et al., 1989; Kornblum, et al., 1990; Eimer, 1993, 1995; Procter, et al., 1995). In contrast, evidence for a perceptual origin of inverse priming results from stimuli which do not overlap with response dimensions (Krüger et al., 2010; Mattler, 2006, 2007). These stimuli might be processed on a different, less automatic route which involves controlled response selection processes (Kornblum, et al., 1990) which eliminates automatic response activation. Further research is needed to examine this hypothesis.

Cognitive control has traditionally been linked to conscious influences on cognitive processing and behavior, which has been strictly separated from automatic processing. However, everyday experiences, may question whether we have to be aware of every stimulus which has to be taken into account to behave successfully for instance when one drives a car in a difficult situation. To account for priming effects of unconscious stimuli, a notion of "conditional automaticity" has been suggested recently which assumes that a specific task set may prepare the processing system in such a way that task relevant stimuli can be processed irrespective of conscious awareness (e.g., Kiefer and Martens, 2010; Sumner and Husain, 2008). However, experimental evidence for the influence of unconscious stimuli on cognitive control processes has been provided only recently. Behavioral priming studies suggested that unconscious primes can induce changes of task sets (Mattler, 2003; 2005; 2007), a finding which has been substantiated by fMRI findings (Lau and Passingham, 2007). Studies using stop-signal and go-nogo paradigms have provided physiological evidence for the effects of unconscious stimuli on control processes in the frontal human cortex (Van Gaal et al., 2008; 
2009; 2010a; 2010b). Finally, studies using the inverse priming paradigm have provided evidence for the influence of unconscious information on inhibitory control operations (Boy, Husain, et al., 2010; Boy, Evans, et al., 2010; Sumner et al., 2007). The present findings extend this line of evidence by showing that small changes from relevant to irrelevant masks leads to a change of control operations which also respond to the effect of unconscious stimuli. Thus, the present findings suggest that a variety of control operations can integrate stimulus information which is not consciously accessible for the behaving subject. 


\section{Acknowledgments}

We thank Peter Dechent and the group MR-Research in Neurology and Psychiatry at the University of Göttingen for allowance and technical support in using the scanner for this study. This research was funded by the Deutsche Forschungsgemeinschaft Grant MA 2276/32 awarded to Uwe Mattler. 


\section{References}

Barch, D.M., Braver, T.S., Akbudak, E., Conturo, T., Ollinger, J., \& Snyder, A. (2001). Anterior cingulate cortex and response conflict: effects of response modality and processing domain. Cerebral Cortex, 11, 837-848.

Böhler, C.N., Appelbaum, L.G., Krebs, R.M., Hopf, J.M., \& Woldorff, M.G. (in press). Pinning down response inhibition in the brain - Conjunction analyses of the Stop-signal task. Neuroimage.

Boy, F., Evans, C.J., Edden, R.A.E., Singh, K.D., Husain, M., \& Sumner, P. (2010). Individual differences in subconscious motor control predicted by GABA concentration in SMA. Current Biology, 20, 1779-1785.

Boy, F., \& Sumner, P. (2010). Tight coupling between positive and reversed priming in the masked priming paradigm. Journal of Experimental Psychology: Human Perception and Performance, 36(4), 892-905.

Boy, F. Husain, M., Singh, K.D., \& Sumner, P. (2010). Supplementary motor area activations in unconscious inhibition of voluntary action. Experimental Brain Research, 206, 441-448.

Caspers, S., Geyer, S., Schleicher, A., Mohlberg, H., Amunts, K., \& Zilles, K. (2006). The human inferior parietal cortex: cytoarchitectonic parcellation and interindividual variability. Neuroimage, 33, 430-448.

Colebatch, J.G. (2007). Bereitschaftspotential and movement-related potentials: origin, significance, and application in disorders of human movement. Movement Disorders, 22(5), 601-610.

Deen, B., Pitskel, N.B., \& Pelphrey, K.A. (in press). Three systems of insular functional connectivity identified with cluster analysis. Cerebral Cortex.

Dehaene, S., Artiges, E., Naccache, L., Martelli, C., Viard, A., Schurhoff, F., and others. (2003). Conscious and subliminal conflicts in normal subjects and patients with schizophrenia: The role of the anterior cingulate. Proceedings of the National Academy of Sciences USA, 100, 13722-13727. 
Dosenbach, N.U.F., Visscher, K.M., Palmer, E.D., Miezin, F.M., Wenger, K.K., Kang, H.C., and others (2006). A Core System for the Implementation of Task Sets. Neuron, 50, 799812.

Dosenbach, N.U.F., Damien, A.F., Miezin, F.M., Cohen, A.L., Wenger, K.K., Dosenbach, R.A.T., and others (2007). Distinct brain networks for adaptive and stable task control in humans. Proceedings of the National Academy of Sciences USA, 104, 11073-11078.

Eickhoff, S.B., Paus, T., Caspers, S., Grosbras, M.-H., Evans, A.C., Zilles, K., \& Amunts, K. (2007). Assignment of functional activations to probabilistic cytoarchitectonic areas revisited. Neuroimage, 36(3), 511-521.

Eimer, M. (1995). Stimulus-response compatibility and automatic response activation: evidence from psychophysiological studies. Journal of Experimental Psychology: Human Perception and Performance, 21(4), 837-854.

Eimer, M. (1999). Facilitatory and inhibitory effects of masked prime stimuli on motor activation and behavioural performance. Acta Psychologica, 101, 293-313.

Eimer, M. \& Schlaghecken, F. (1998). Effects of masked stimuli on motor activation: Behavioral and electrophysiological evidence. Journal of Experimental Psychology: Human Perception and Performance, 24, 1737-1747.

Eimer, M., \& Schlaghecken, F. (2002). Links between conscious awareness and response inhibition: Evidence from masked priming. Psychonomic Bulletin \& Review, 9, 514-520.

Eimer, M. \& Schubö, A., \& Schlaghecken, F. (2002). Locus of inhibition in the masked priming of response alternatives. Journal of Motor Behaviour, 34, 3-10.

Goebel, R., Khorram-Sefat, D., Muckli, L., Hacker, H., \& Singer, W. (1998). The constructive nature of vision: direct evidence from functional magnetic resonance imaging studies of apparent motion and motion imagery. European Journal of Neuroscience, 10, 1563-1573.

Hazeltine, E., Poldrack, R., \& Gabrieli, J.D.E. (2000). Neural activation during response conflict. Journal of Cognitive Neuoscience, 12, 118-129.

Huber, D.E. (2008). Immediate priming and cognitive aftereffects. Journal of Experimental Psychology: General, 137, 324-347. 
Jaśkowski, P. (2007a). The effect of nonmasking distractors on the priming of motor responses. Journal of Experimental Psychology: Human Perception and Performance, 33, 456-468.

Jaśkowski, P. (2007b). How important is the prime's gestalt for subliminal priming? Consciousness \& Cognition, 16, 485-497.

Jaśkowski, P. (2008). The negative compatibility effect with nonmasking flankers: A case for mask-triggered inhibition hypothesis. Consciousness and Cognition, 17, 765-777.

Kiefer, M., \& Martens, U. (2010). Attentional Sensitization of Unconscious Cognition: Task Sets Modulate Subsequent Masked Semantic Priming. Journal of Experimental Psychology: General, 139, 464-489.

Klapp, S. T., \& Hinkley, L. B. (2002). The negative compatibility effect: Unconscious inhibition influences reaction time and response selection. Journal of Experimental Psychology: General, 131, 255-269.

Kornblum, S., Hasbroucq, T., \& Osman, A. (1990). Dimensional overlap: cognitive basis for stimulus-response compatibility - A model and taxonomy. Psychological Review, 97(2), 253-270.

Koski, L., \& Paus, T. (2000). Functional connectivity of the anterior cingulate cortex within the human frontal lobe: a brain-mapping meta-analysis. Experimental Brain Research, $133,55-65$.

Krüger, D., Klapötke, S., \& Mattler, U. (2011). PRP-paradigm provides evidence for a perceptual origin of the negative compatibility effect. Consciousness and Cogntion, 20, 866-881.

Leuthold, H. (2011). The simon effect in cognitive electrophysiology: A short review. Acta Psychologicy, 136, 203-211.

Lleras, A. \& Enns, J.T. (2004). Negative compatibility or object updating? A cautionary tale of mask-dependent priming. Journal of Experimental Psychology: General, 133, 475-493.

Lleras, A. \& Enns, J.T. (2005). Updating a cautionary tale of masked priming: Reply to Klapp (2005). Journal of Experimental Psychology: General, 134, 436-440. 
Lleras, A., \& Enns, J. T. (2006). How much like a target can a mask be? Geometric, spatial, and temporal similarity in priming: A reply to Schlaghecken and Eimer (2006). Journal of Experimental Psychology: General, 135, 495-500.

Malikovic, A., Amunts, K., Schleicher, A., Mohlberg, H., Eickhoff, S.B., Wilms, M., Palomero-Gallagher, N., Armstrong, E., Zilles, K. (2007). Cytoarchitectonic Analysis of the Human Extrastriate Cortex in the Region of V5/MT1: A Probabilistic, Stereotaxic Map of Area hOc5. Cerebral Cortex, 17, 562-574.

Matsuzaka, Y., \& Tanji, J. (1996). Changing directions of forthcoming arm movements: Neuronal activity in the presupplementary and supplementary motor area of the monkey cerebral cortex. Journal of Neurophysiology, 76(4), 2327-2342.

Mattler, U. (2003). Priming of mental operations by masked stimuli. Perception and Pschophysics, 65, 167-187.

Mattler, U. (2005). Inhibition and decay of motor and non-motor priming. Perception and Pschophysics, 67, 285-300.

Mattler, U. (2006). On the locus of priming and inverse priming effects. Perception and Psychophysics, 68, 975-991.

Mattler, U. (2007). Inverse target- and cue-priming effects of masked stimuli. Journal of Experimental Psychology: Human Perception and Performance, 33, 83-102.

Mummery et al., 1998 C.J. Mummery, K. Patterson, J.R. Hodges and C.J. Price, (1998). Functional neuroanatomy of the semantic system: divisible by what?, J. Cog. Neurosci. $10,766-777$.

Nachev, P., Kennard, C., \& Husain, M. (2008). Functional role of the supplementary and presupplementary motor areas. Nature Reviews Neuroscience, 9, 856-869.

Norman, D.A., \& Shallice, T. (1986). Attention to action: Willed and automatic control of behavior. In R.J. Davidson, G.E. Schwartz, \& D. Shapiro (Eds.), Consciousness and selfregulation, Vol. 4. New York: Plenum Press, pp. 1-18.

Picard, N., \& Strick, P.L. (1996). Motor areas of the medial wall: a review of their location and function. Cerebral Cortex, 6, 342-353. 
Picard, N., \& Strick, P.L. (2001). Imaging the premotor areas. Current Opinion in Neurobiology, 11, 663-672.

Proctor, R.W., Lu.C.-H., Wang, H., \& Dutta, A. (1995). Activation of response codes by relevant and irrelevant stimulus information. Acta Psychologica, 90, 275-286.

Rees, G., Kreiman, G., \& Koch, C. (2002) Neural correlates of consciousness in humans. Nature Review Neuroscience, 3, 261-270.

Roger, C., Bénar, C.G., Vidal, F., Hasbroucq, T., \& Burle, B. (2010). Rostral Cingulate Zone and correct response monitoring: ICA and source localization evidences for the unicity of correct- and error-negativities. Neuroimage, 51, 391-403.

Schlaghecken, F., \& Eimer, M. (2000). A central-peripheral asymmetry in masked priming. Perception \& Psychophysics, 62, 1367-1382.

Schlaghecken, F., \& Eimer, M. (2006). Active masks and active inhibition: A comment on Lleras and Enns (2004) and on Verleger, Jaśkowski, Aydemir, van der Lubbe, and Groen (2004). Journal of Experimental Psychology. General, 135, 484-494.

Shepard, R.N., \& Zare, S.L. (1983). Path-guided apparent motion, Science, 220, 632-634.

Sohrabi, A. \& West, R.L. (2009). Positive and negative congruency effects in masked priming: A neuro-computational model based on representation, attention and conflict. Brain Research, 1289, 124-132.

Sumner, P., Nachev, P., Morris, P., Peters, A.M., Jackson, S.R., Kennard, C., \& Husain, M. (2007). Human medial frontal cortex mediates unconscious inhibition of voluntary action. Neuron, 54(5), 697-711.

Swick, D., Ashley, V., \& Turken, U. (2011). Are the neural correlates of stopping and not going identical? Quantitative meta-analysis of two response inhibition tasks, Neuroimage, $56,1655-1665$.

Tzourio-Mazoyer, N., Landeau, B., Papathanassiou, D., Crivello, F., Etard, O., Delcroix, N., Mazoyer, B., \& Joliot, M. (2002). Automated anatomical labeling of activations in SPM using a macroscopic anatomic parcellation of the MNI MRI single-subject brain. Neuroimage, 15, 273-289. 
Van Gaal, S., Ridderinkhof, K.R., Fahrenfort, J.J., Scholte, S., \& Lamme, V.A.F. (2008). Frontal cortex mediates unconsciously triggered inhibitory control. Journal of Neuroscience, 28, 8053-8062.

Van Gaal, S., Ridderinkhof, K.R., Scholte, S., \& Lamme, V.A.F. (2010). Unconscious activation of the prefrontal No-Go network. Journal of Neuroscience, 30, 4143-4150.

Van Gaal, S., Ridderinkhof, K.R., Van den Wildenberg, W.P.M., \& Lamme, V.A.F. (2009). Dissociating consciousness from inhibitory control: Evidence for unconsciously triggered response inhibition in the stop signal task. Journal of Experimental Psychology: Human Perception and Performance, 35, 1129-1139.

Van Gaal, S., Scholte, S., Lamme, V.A.F., Fahrenfort, J.J., \& Ridderinkhof, K.R. (2010). Pre-SMA gray-matter density predicts individual differences in action selection in the face of conscious and unconscious response conflict. Journal of Cognitive Neuroscience, 23, 382-390.

Van Gaal, S., Lamme, V.A.F., Fahrenfort, J.J., \& Ridderinkhof, K.R. (2010b). Dissociable Brain Mechanisms Underlying the Conscious and Unconscious Control of Behavior. Journal of Cognitive Neuroscience, 23, 91-105.

Vorberg, D., Mattler, U., Heinecke, A., Schmidt, T., \& Schwarzbach, J. (2003). Different time courses for visual perception and action priming. Proceedings of the National Academy of Sciences USA, 100, 6275-6280.

Wilms, M., Eickhoff, S.B., Specht, K. ,Amunts, K., Shah, N.J., Malikovic, A., \& Fink, G.R. (2005). Human V5/MT+: comparison of functional and cytoarchitectonic data. Anatomy and Embryology, 210(5-6), 485-495. 
Table 1. Main effect of SOA on incongruent trials

\begin{tabular}{|c|c|c|c|c|c|c|c|}
\hline \multirow[t]{2}{*}{ Anatomical region } & \multirow[t]{2}{*}{ Hemisphere } & \multirow[t]{2}{*}{ BA } & \multirow[t]{2}{*}{$\begin{array}{l}\text { Cluster } \\
\text { size }\end{array}$} & \multirow[t]{2}{*}{$t$ value } & \multicolumn{3}{|c|}{$\begin{array}{c}\text { MNI } \\
\text { coordinates }\end{array}$} \\
\hline & & & & & $\mathbf{x}$ & y & $\mathbf{z}$ \\
\hline \multicolumn{8}{|l|}{ Relevant Mask } \\
\hline inferior temporal gyrus & $\mathbf{R}$ & & 65 & 5.6 & 46 & -66 & -6 \\
\hline middle occipital gyrus & $\mathrm{R}$ & & & 4.1 & 46 & -74 & 2 \\
\hline Middle temporal gyrus & $\mathrm{R}$ & & & 3.6 & 42 & -64 & 4 \\
\hline $\begin{array}{l}\text { Middle occipital gyrus } \\
\text { Irrelevant Mask }\end{array}$ & $\mathbf{L}$ & & 73 & 5.4 & -44 & -74 & -2 \\
\hline inferior temporal gyrus & $\mathbf{R}$ & & 300 & 5.6 & 42 & -56 & -10 \\
\hline Middle temporal gyrs & $\mathrm{R}$ & & & 5.5 & 50 & -66 & 8 \\
\hline Fusiform gyrus & $\mathbf{L}$ & & 22 & 5.2 & -36 & -56 & -16 \\
\hline middle occipital gyrus & $\mathbf{R}$ & & 52 & 5.1 & 32 & -90 & 10 \\
\hline Inferior occipital gyrus & L & & 251 & 5.0 & -44 & -72 & -6 \\
\hline Fusiform gyrus & L & & & 5.0 & -30 & -66 & -4 \\
\hline middle occipital gyrus & $\mathrm{L}$ & & & 4.7 & -36 & -74 & 0 \\
\hline Fusiform gyrus & $\mathbf{L}$ & & 13 & 4.6 & -30 & -58 & -4 \\
\hline middle occipital gyrus & $\mathbf{L}$ & & 27 & 4.6 & -32 & -82 & 12 \\
\hline middle occipital gyrus & $\mathrm{L}$ & & & 4.1 & -34 & -86 & 22 \\
\hline Linual gyrus & $\mathbf{R}$ & $18(30 \%)$ & 15 & 4.4 & 24 & -66 & 0 \\
\hline Superior temporal gyrus & $\mathbf{R}$ & & 10 & 4.4 & 64 & -30 & 14 \\
\hline Inferior occipital gyrus & $\mathbf{R}$ & & 33 & 4.1 & 36 & -76 & -10 \\
\hline Calcarine gyrus & $\mathbf{R}$ & $18(50 \%)$ & 13 & 4.0 & 8 & -70 & 18 \\
\hline
\end{tabular}


Table 3. Brain areas specifically involved in control operations with relevant masks and both masks.

\begin{tabular}{|c|c|c|c|c|c|c|c|}
\hline \multirow[t]{2}{*}{ Anatomical region } & \multirow[t]{2}{*}{ Hemisphere } & \multirow[t]{2}{*}{ BA } & \multirow[t]{2}{*}{$\begin{array}{c}\text { Cluster } \\
\text { size }\end{array}$} & \multirow[t]{2}{*}{$t$ value } & \multicolumn{3}{|c|}{$\begin{array}{c}\text { MNI } \\
\text { coordinates }\end{array}$} \\
\hline & & & & & $\mathbf{x}$ & $\mathbf{y}$ & $\mathbf{z}$ \\
\hline \multicolumn{8}{|l|}{ Relevant Mask only } \\
\hline Supplementary motor area & $\mathbf{R}$ & $6(50 \%)$ & 13 & 5.8 & 12 & -8 & 64 \\
\hline Insula lobe & $\mathbf{R}$ & & 12 & 5.2 & 30 & 24 & 8 \\
\hline Insula lobe & $\mathbf{R}$ & & 77 & 5.0 & 38 & 2 & 10 \\
\hline Insula lobe & $\mathrm{R}$ & & & 4.5 & 46 & 0 & 6 \\
\hline Middle cingulate cortex & $\mathbf{R}$ & & 48 & 4.7 & 10 & 10 & 38 \\
\hline Middle cingulate cortex & $\mathrm{L}$ & $6(10 \%)$ & & 4.0 & 0 & 6 & 40 \\
\hline Middle cingulate cortex & $\mathrm{L}$ & & & 3.8 & -8 & 6 & 36 \\
\hline Cerebellum & $\mathbf{R}$ & & 27 & 4.6 & 18 & -54 & -14 \\
\hline Rolandic Operculum & $\mathbf{R}$ & & 11 & 4.6 & 48 & -24 & 22 \\
\hline Rolandic Operculum & $\mathbf{L}$ & & 14 & 4.5 & -44 & -4 & 8 \\
\hline Supramarginal Gyrus & $\mathbf{L}$ & & 24 & 4.5 & -50 & -32 & 30 \\
\hline Postcentral Gyrus & $\mathbf{L}$ & & 30 & 4.1 & -60 & -18 & 18 \\
\hline \multicolumn{8}{|l|}{ Both masks } \\
\hline Supplementary motor area & $\mathbf{R}$ & $6(70 \%)$ & 13 & 4.1 & 2 & -8 & 52 \\
\hline
\end{tabular}

Note. Activation in these areas was stronger with long as compared to short SOA on congruent trials but not on incongruent trials, exclusively on trials with relevant masks or with both masks, respectively. Note that we failed to find any brain region which was exclusively involved in control operations with irrelevant masks. Only regions above the threshold of $\mathrm{p}<$ .001 and the minimum cluster size of $\mathrm{k}>10$ are reported. Cluster maxima are printed in bold face. BA is the Brodman Area nearest to the coordinates. Within parenthesis the probability for this assignment is also reported as determined by the anatomical toolbox (Version 1.6, Eickhoff, 2007). 


\section{Figures Legends}

Figure 1. Stimulus sequence used in the experiment. Primes were followed by either a relevant or an irrelevant mask which in turn was followed by the target stimulus. Primes and targets were either congruent or incongruent. An incongruent trial is depicted. With a masktarget SOA of $33 \mathrm{~ms}$ positive priming was expected while with $150 \mathrm{~ms}$ inverse priming should occur.

Figure 2. A) Response activation model for inverse priming. The model is based on the accumulator model of Vorberg et al. (2003) and research demonstrating the time course of lateralized readiness potentials in inverse priming (Eimer and Schlaghecken, 1998; Seiss and Praamstra, 2005). The figure describes the time course of the activation difference of left and right hand responses. Three consecutive phases can be distinguished: first, the prime activates the associated response. Second, the presentation of the mask induces a response activation which reverses the prime-related response activation. The mask induced activation drives the differential response activation away from the prime associated response until the target is presented. Third, targets induce response activation. When prime and target stimuli are congruent, the differential response activation is reversed again. Prime-incongruent targets, however, simply continue the mask induced activation. The model assumes that an overt response is triggered when the differential response activation reaches a threshold c. We assume that reversals of response activation induce response conflicts which require regulatory demands. Response conflict increases with long mask-target SOA on congruent trials leading to higher regulatory demands than on incongruent trials. Therefore, neural activation which increases with SOA on congruent trials but not on incongruent trials reflects the increase of regulatory demands in inverse priming.

B) Predicted effects of SOA and regulatory demands on brain activation. Left panel: brain activation in a brain region which is modulated by SOA in the same way on congruent and incongruent trials reflects differences in perceptual processing which result from physical differences in the stimulus sequence with long and short SOA. Middle panel: brain activation in regions which respond differentially to SOA on congruent and incongruent trials with a larger SOA effect on congruent than on incongruent trials. Activation in these regions is related to both, perceptual and control operations. Right panel: brain activation in a region which responds to SOA only on congruent but not on incongruent trials. Activation in these regions reflects the increased processing of control operations on congruent trials with long 
SOA. Note, the model predicts a very specific interaction of SOA and Congruency as a sign of brain areas which are involved in control operations.

Figure 3. Behavioral Results. Net priming effect for RTs (lines) and error rates (bars) at short and long SOA separate for the relevant (red) and irrelevant mask (blue). Priming effects are determined as the difference in performance measures on incongruent trials minus congruent trials. While short mask-target SOAs led to positive priming effects of comparable magnitude for both masks, long SOAs produced inverse priming which was larger and significantly below zero for relevant masks.

Figure 4. Main effect of SOA on brain activation. Activations on trials with relevant masks (red), irrelevant masks (blue), and overlapping activation for both masks (purple). Results underscore the validity of the response activation model. A) Incongruent trials: SOA affected only visual areas. B) Congruent trials: SOA affected the same areas as on incongruent trials plus a number of additional areas within the parietal and frontal cortex. C) Common main effect of SOA on both, congruent and incongruent trials: Visual effects peaked within V5 / $\mathrm{MT}+$. All statistical results were thresholded at $\mathrm{p}<.001$ (uncorrected) and only clusters of $\mathrm{k}>$ 10 adjacent voxels are shown. We excluded voxels which showed stronger SOA effects on congruent as compared to incongruent trials thresholded by the default SPM exclusion threshold of $\mathrm{p}<.05$ (uncorrected).

Figure 5. Brain areas which are exclusively involved in control operations. Brain activations on trials with relevant masks (red) and on trials with either type of mask (purple). Activity in these brain areas increased with SOA on congruent trials but not on incongruent trials. These areas were determined by a significant SOA effect on congruent trials and a significant interaction of Congruency and SOA excluding brain areas with a significant SOA effect on incongruent trials. To find brain regions which are exclusively involved in mask specific control processes, we also excluded brain areas which showed either an effect of SOA on congruent trials or the interaction Congruency x SOA on trials with the opposite type of mask. Nine brain areas showed the specific pattern of activity which signifies control operations exclusively with relevant masks. Only SMA activity showed the pattern for either mask. Bar plots depict mean betas per condition averaged across subjects. Error bars indicate standard error across subjects. Thresholds are the same as in Figure 4. Coordinates refer to MNI-space.

Figure 6. Correlation Analysis between the SOA effect on congruent trials in the SMA and behavioral inverse priming effects. A) Scatterplot illustrating the relation between individuals' 
SOA effect in the SMA on congruent trials and their behavioral inverse priming effect separated for the relevant (blue) and irrelevant masks (red). Activity was determined in the SMA voxel which was maximally affected by SOA variation on congruent trials. We obtained a significant negative correlation for both, relevant $(r=-.43, p<.05)$ and irrelevant masks $(r=$ $-.50, \mathrm{p}<.05)$. B) Distribution of correlation coefficients in those SMA voxels which respond to SOA on congruent trials, separated for trials with relevant (blue) and irrelevant masks (red). Correlations smaller than the value indicated by the vertical dashed line are significant at $\mathrm{p}<.05$. 
Figure 1

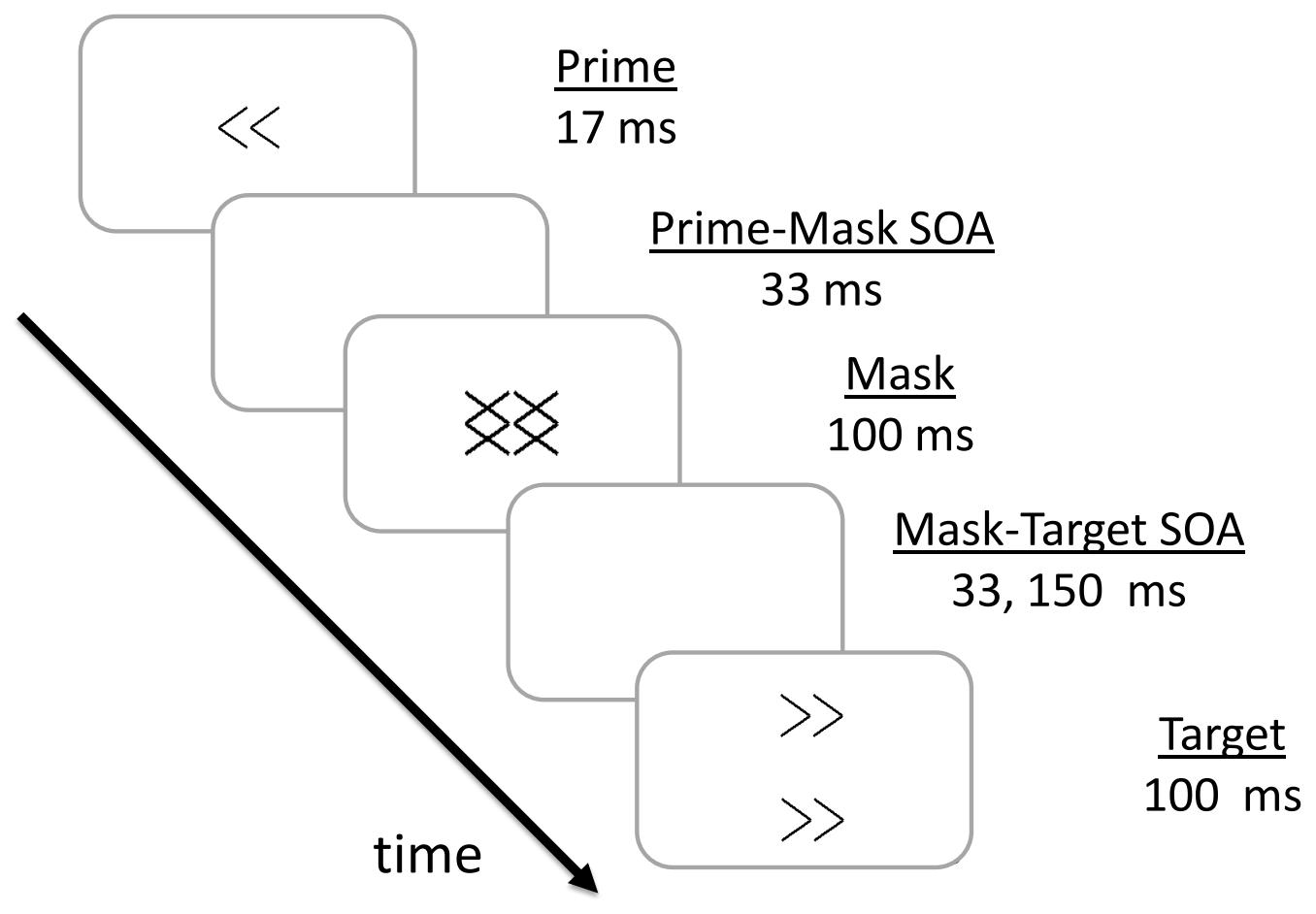


Figure 2

A
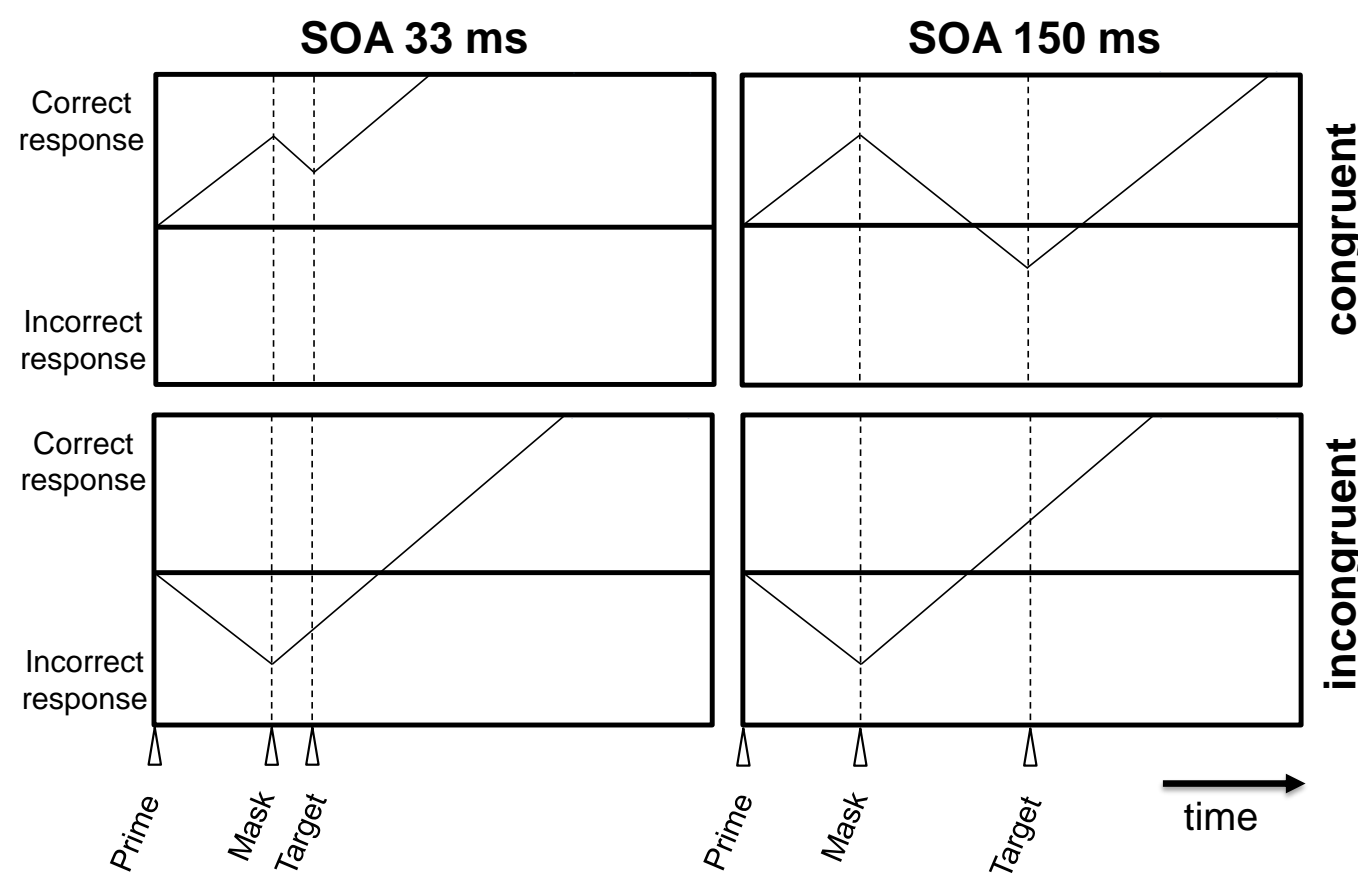

B

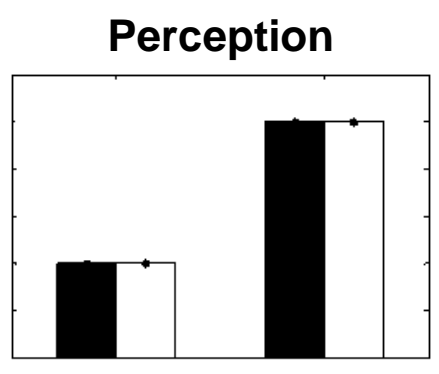

33

150

Mask-Target SOA [ms]

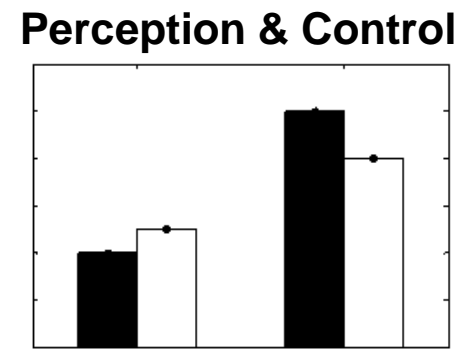

33

150

Mask-Target SOA [ms]

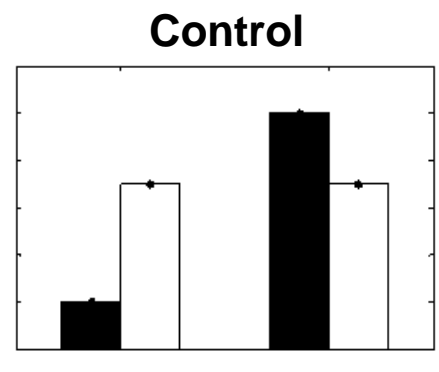

33

150

Mask-Target SOA [ms]

congruent

incongruent 
Figure 3

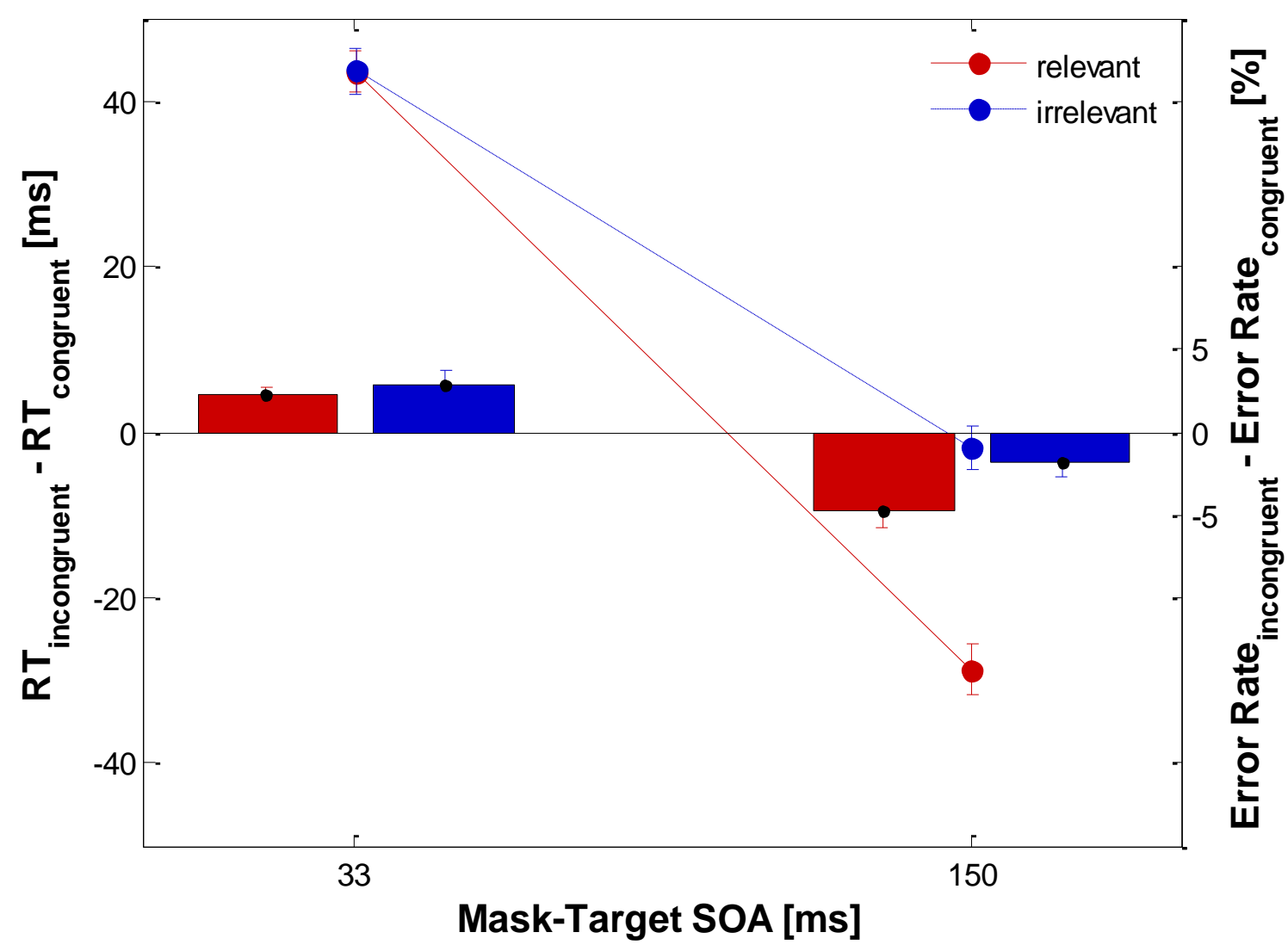


Figure 4

A Inc: $S O A=150>S O A=33$
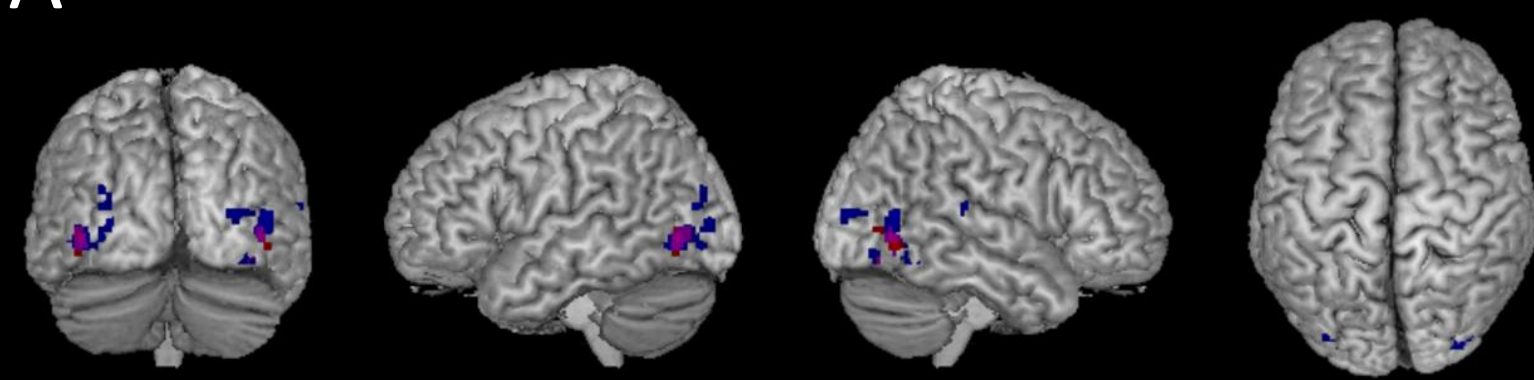

B Con: $S O A=150>S O A=33$
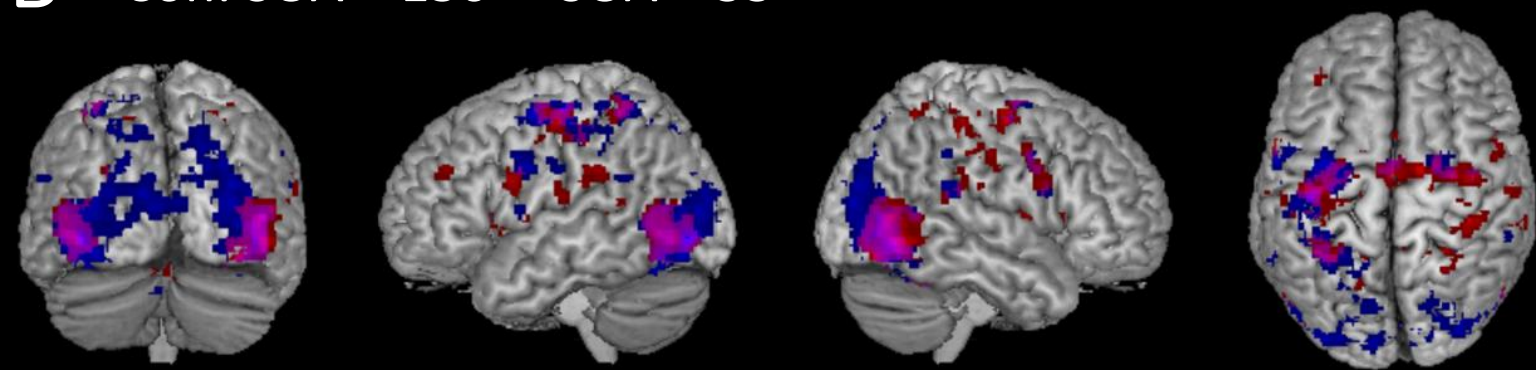

C $[$ Con: $\mathrm{SOA}=150>\mathrm{SOA}=33] \&[$ Inc: $\mathrm{SOA}=150>\mathrm{SOA}=33]$
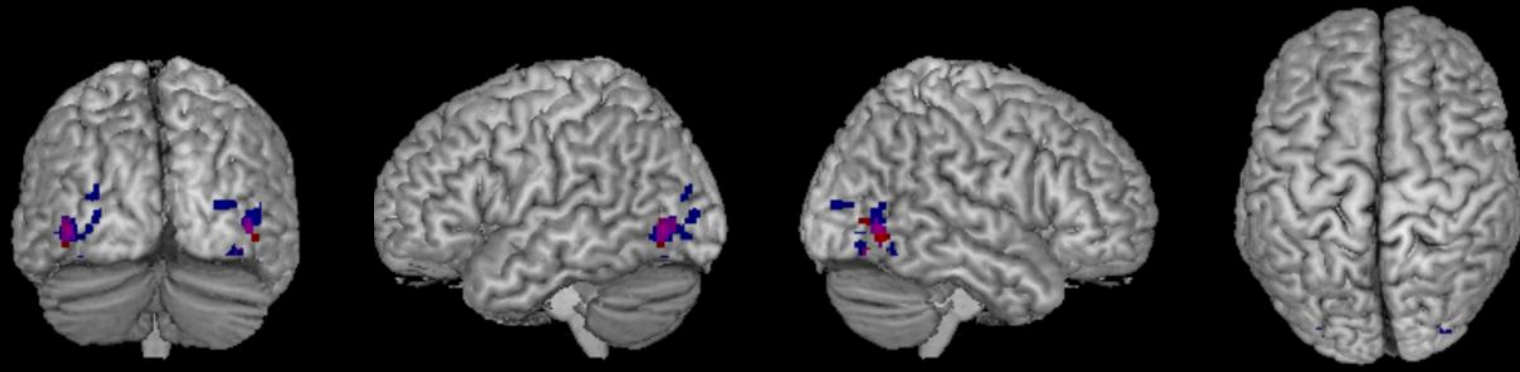

Relevant mask

T-values

Irrelevant mask

Both masks 
Figure 5

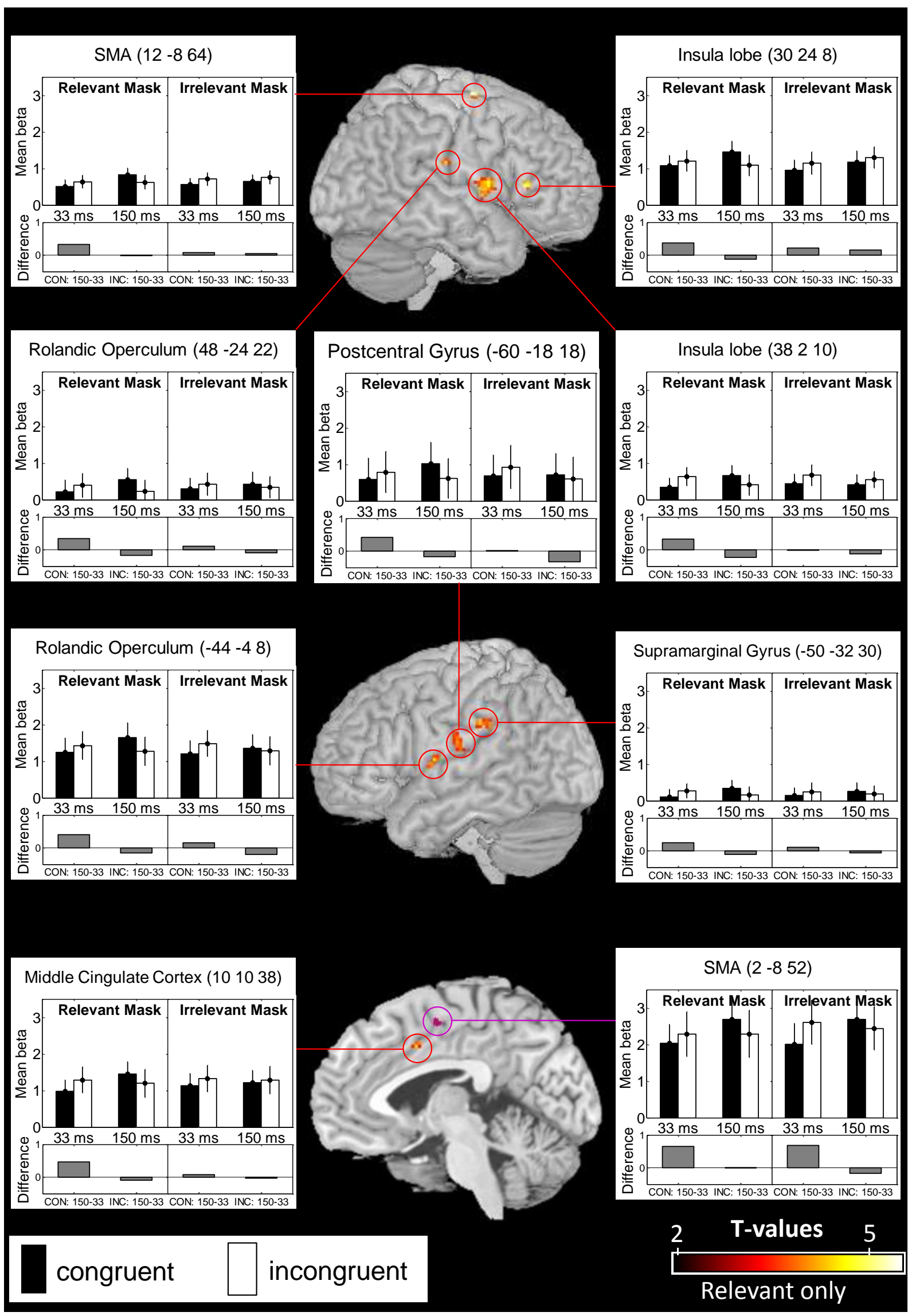


Figure 6
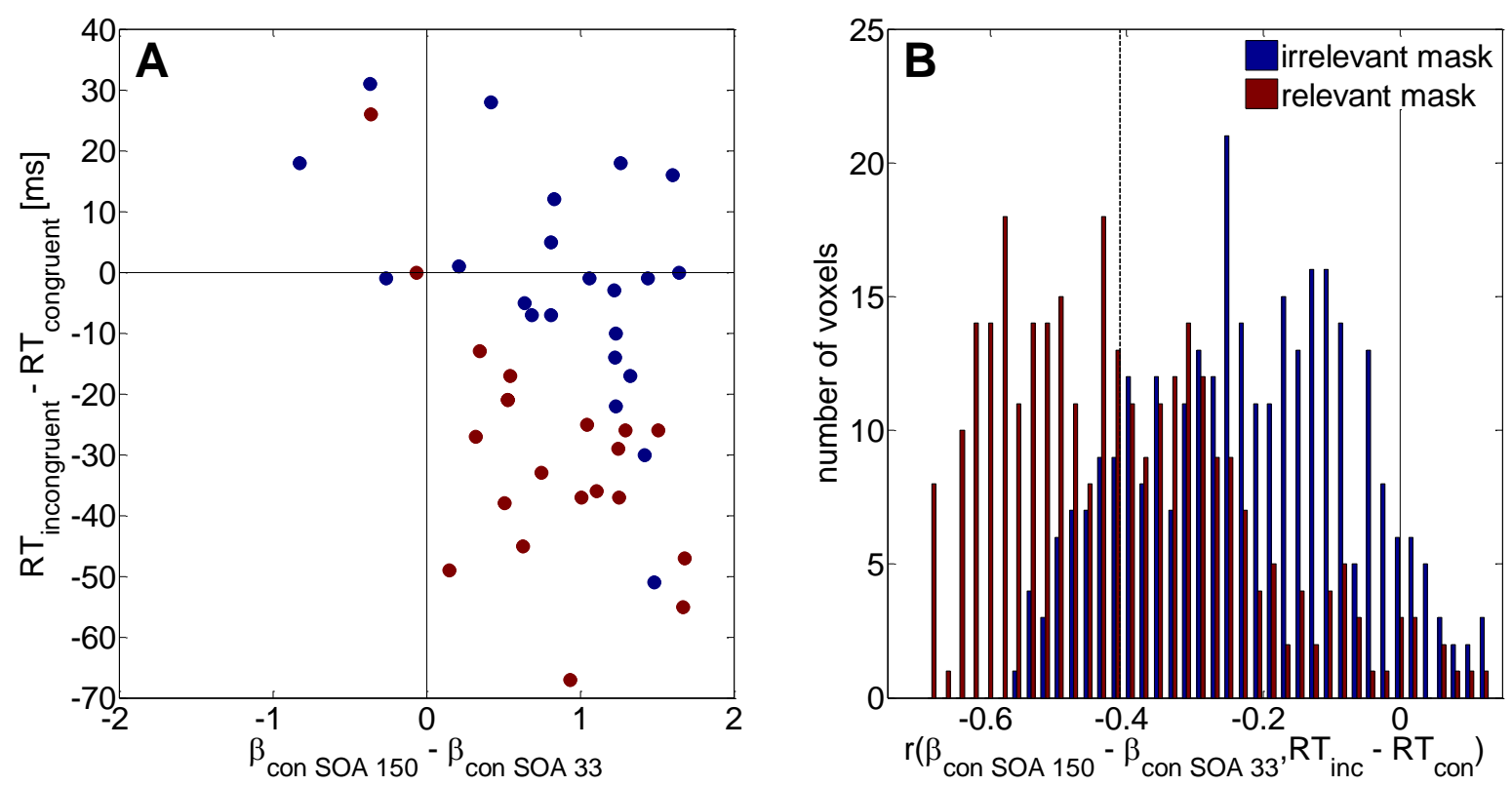


\section{List of Figures}

Fig. 1 Stimulus dimensions determining inverse priming effects 16

Fig. 2 Stimuli used in Project II 22

Fig. 3 Stimuli used in Project III 26

Fig. 4 Stimuli used in Project IV 31

Fig. $5 \quad$ Response activation model of inverse priming 34

Fig. 6 Priming effects and prime visibility with irrelevant masks 47 


\section{List of Abbreviations}

fMRI Functional magnetic resonance imaging

LRP Lateralized readiness potential

RLM Random line mask

PRP Psychological Refractory Period

SMA Supplementary motor area

SOA Stimulus onset asynchrony

SR Stimulus-response

RT Response time

rTMS Repetitive transcranial magnetic stimulation 


\section{Curriculum Vitae}

Persönliche Daten

\begin{tabular}{ll}
\hline Name: & Daniel Krüger \\
Geburtsdatum: & 08.07.1982 in Steinheim, Westfalen \\
& \\
Dienstliche & Daniel Krüger \\
Anschrfit: & Georg-August-Universität Göttingen \\
& Georg-Elias-Müller-Institut für Psychologie \\
& Abteilung für Experimentelle Psychologie \\
& Goßlerstr. 14 \\
& 37073 Göttingen \\
& Email: dkruege@ uni-goettingen.de \\
& Tel: 0551 - 39 3616
\end{tabular}

Private Anschrift: Daniel Krüger

Comeniusstr. 3

38102 Braunschweig

\section{Ausbildung}

$06 / 2002$

$10 / 2002-09 / 2003$

$10 / 2003-07 / 2008$

$10 / 2005$

$07 / 2008$

$05 / 2005-12 / 2007$

Seit 07/2008
Allgemeine Hochschulreife, Gymnasium Leopoldinum, Detmold

Studium Technomathematik, Universität Paderborn

Studium Psychologie, Technische Universität Braunschweig

Vordiplom Psychologie

Diplom Psychologie

Diplomarbeit: „Response Priming and the role of conscious prime perception - an fMRI study“"

Diverse Tätigkeiten als Studentische Hilfskraft in Forschung und Lehre

Wissenschaftlicher Mitarbeiter in der Abteilung für Experimentelle Psychologie, Georg-Elias-Müller Institut für Psychologie, GeorgAugust Universität Göttingen 